UCRL-CR-115387

P.O. B108511

\title{
Cubic to Tetragonal Crystal Lattice Reconstruction During Ordering or Decomposition
}

\author{
Byung-kl Cheong
}

September 1, 1992

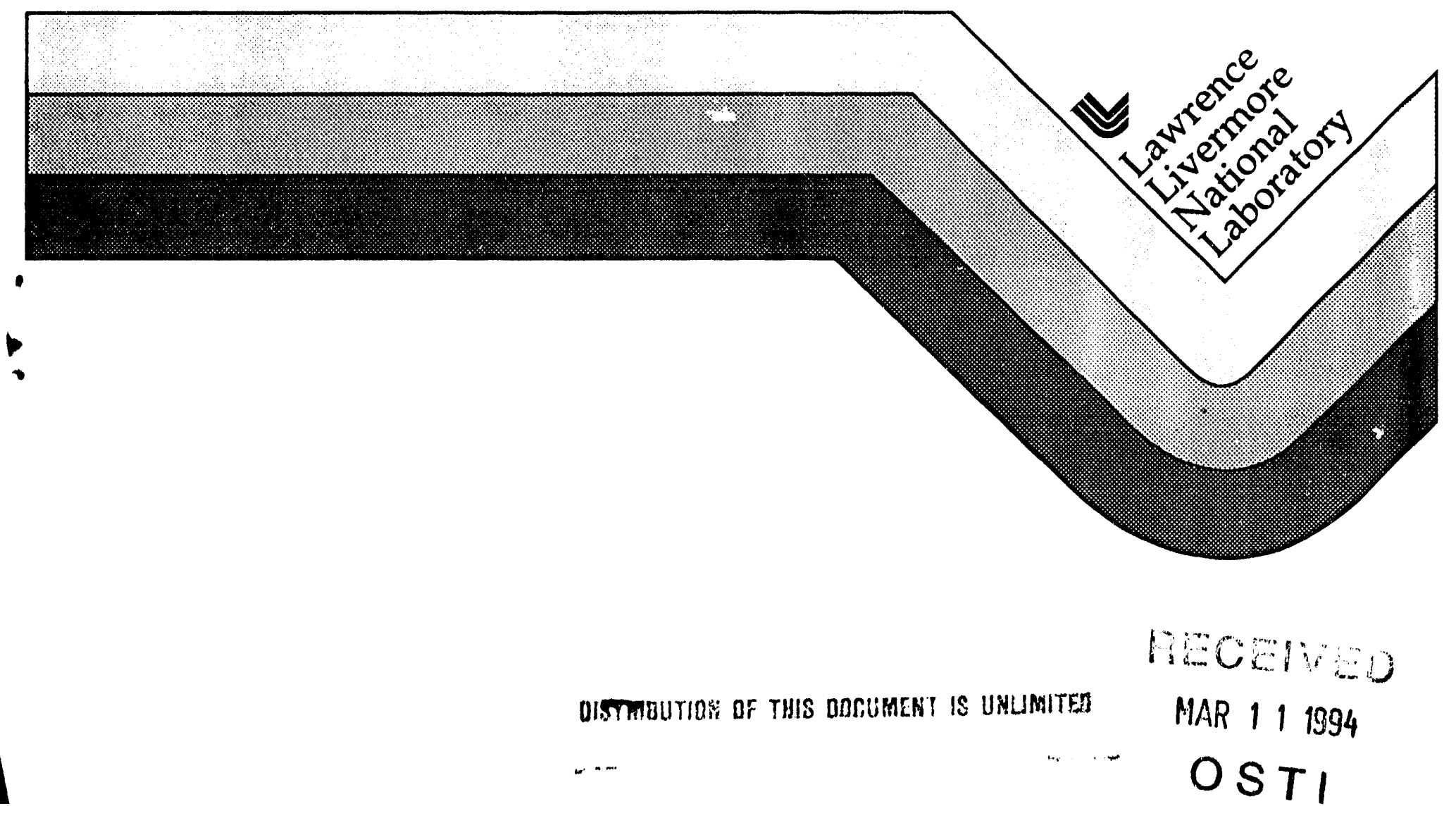




\section{DISCLAIMER}

Work performed under the auspices of the U.S. Department of Energy by Iawrence Livermore Vational Laboratory under contract number W-7405-E.VG-48.

This document was prepared as an account of work sponsored by an agency of the United Siates Government. Neither the United States Government nor the University of California nor any of their employees, makes any warranty, express or implied, or assumes any legal liability or responsibility for the accuracy, completeness. or usefulness of any information. apparatus, product, or process disclosed, or represents that its use would not infringe privately owned rights. Reference herein to any specific commercial products, process, or service by trade name. trademark, manufacturer, or otherwise, does not necessarily constitute or imply its endorsement, recommendation, or favoring by the United States Covernment or the University of California. The vieus and opinions of authors expressed herein do not necessarily state or reflect those of the Lnited States Government or the Iniversity of California, and shall not be used for advertising or product endorsement purposes. 


\title{
Cubic to Tetragonal Crystal Lattice Reconstruction During Ordering or Decomposition
}

\author{
Byung-kI Cheong \\ Department of Materials Science and Engineering \\ Carnegie Mellon University \\ Pittsburgh, Pennsylvania \\ September 1, 1992
}

\begin{abstract}
Submitted to Carnegie Institute of Technology
in partial fulfillment of the requirements for the degree of Doctor of Philosophy in Materials Science and Engineering
\end{abstract}


We have investigated the thermodynamic stability and the morphology of product phases in diffusional phase transformations which involve cubic to tetragonal crystal lattice reconstructions. Two different kinds of diffusional transformations were examined: $\mathrm{LI}_{0}$ ordering (involves fcc to fct lattice change) and decomposition of off-stoichiometric B2 ordering alloys accompanying bcc to fcc Bain transformation. In the first case, the crystal lattice reconstruction is intrinsic to the diffusional atomic reconfiguration process (ordering) whereas it is not intrinsic to but interplays with the concurrent atomic reconfiguration process (compositional separation) in the second case.

In an attempt to understand the thermodynamic consequences of the tetragonal distortion accompanying the $L \mathcal{1}_{0}$ ordering, we have conducted a theoretical investigation by treating the problem of a rigid lattice free energy model. The problem was treated as an elasticity problem and a generalized formulation was proposed in a form which can be directly utilized to study incoherent two phase equilibria. By use of the SCW mean field model as a rigid lattice free energy model, the formulation was applied to the case of our interest. We have shown that the thermodynamic stability of the L1, phase may be significantly influenced by the tetragonal lattice distortion, depending on the magnitude of the associated negative strain energy relative to the competing positive entropic contribution in the free energy of the stress free L1 $1_{0}$ phase. In association with this, a suggestion was made that the neglect of the tetragonal distortion (or use of a rigid lattice free energy model) could be a source of serious errors particularly for alloys with lower $\mathrm{L}_{0}$ ordering transition temperatures (e.g. CuAu and InMg). A prototype phase diagram of the $<001>i c c$ special point structures, calculated with the tetragonal distortion taken into account, has indeed displayed topological features which are fundamentally different from those of a rigid lattice phase diagram and, furthermore, has reproduced the main topological features of the $\mathrm{AuCu}-\mathrm{Au}_{3} \mathrm{Cu}$ and the $\operatorname{lnMg}-\mathrm{In}_{3} \mathrm{Mg}$ side of the respective phase diagram.

In order to also examine the effects of the tetragonal distortion on the morphology of the L1. phase, a TEM investigation was conducted by use of the $\mathrm{Fe}-45$ at.\%Pd alloys. It was 
shown that essential morphological features such as the variant habit plane, the variant volume fraction and the shape of a variant plate are determined so as to best accommodate the tetragonal distortion accompanying the $L 1_{0}$ ordering. For the variant habit planes, we have unequivocally shown that the $\{110\}$ planes are the optimum habit planes. As for the variant volume fraction, we have observed that, initially from a three variant state, the variant volume fraction in a local region varies gradually through a two variant state until a near mono-variant state is reached. Existing theories of polytwin morphology shed little light on this characteristic sequential change. We have explained this in terms of a synergistic interplay between the variant volume fraction and the constraining ability of the ordering surroundings. As regards the shape of a variant plate, we have found that the optimum shape is loop-like with no faceting. By use of an elasticity theory of the shape of a coherent plate-like inclusion, a calculation was made to show a good agreement with the experimental result.

Bain strain relaxation during decomposition of the hyper-eutectoid Cu-9.04 wt.\%Be alloy was studied by TEM and experimental findings were interpreted on the basis of the elasticity theory of a plate-like precipitate. The major feature of the Bain strain relaxation was found that it occurrs gradually along a decomposition path and through the interplay of two basic mechanisms, that is, habit plane rotation and the formation of polytwin domains. Depending on the kinetic path in the initial stages of the decomposition, these two mechanisms interplay in different manners to give rise to two kinds of precipitate morphology: for quenched/aged alloys, single domain plates with globally rotated habit planes which are arranged into a macroscopic polytwin morphology and for continuously cooled alloys, saw-tooth type polytwin plates with segment-wise rotated habit planes. It was shown that, as opposed to the theoretical polytwin plate, a poly-twin plate with the saw-tooth morphology does not satisfy the invariant plane strain condition hence is not stress-free. From analysis of conventional TEM and HRTEM micrographs of the as-quenched states, a formation mechanism of the saw-tooth polytwin plate was proposed in terms of a dislocation model. The proposed model consists of the generation of a twinning dislocation due to intersection of plates and the subsequent alternating glide and climb motion of the dislocation. 


\section{Acknowledgements}

I am deeply grateful to my advisor Prof. D. E. Laughlin for his assistance, encouragement and trust throughout the course of my graduate study at Camegie Mellon. I would like to thank my thesis committee, Prof. W. C. Johnson, Prof. M. J. Readey and Prof. W. A. Soffa of Univ. of Pittsburgh for their valuable comments on the manuscript.

My gratitude to Prof. A. G. Khachaturyan of Rutgers Univ. can not be adequately expressed. Not only has he inspired me profoundly through his numerous important works, but he has also graciously spared his time for many invaluable discussions and for critical review of portions of the manuscript. I am also grateful to Prof. H. I. Aaronson, who is now at Naval Research Lab., for helpful discussions on a part of the thesis work and for his encouragement. To Prof. J. M. Howe of Univ. of Virginia, I would like to extend gratitude for his help on the High Resolution TEM observation of CuBe alloys. I also wish to thank Dr. K. Hono whose unpublished experimental work paved the way for my own investigation of CuBe alloys. My continuing thank to Mr. T. N. Nuhfer for his technical assistance in TEM operation.

I would like to acknowledge the Lawrence Livermore National Lab. and the Brush Wellman Inc. for the research grants which have fueled this work to fruition. Ministry of Education of Korean Government is also acknowledged for the fellowship during initial period of my graduate study.

I would like to take this oppurtunity to express my heartfelt thanks to those I have come to know during my placement in the Department and in Pittsburgh. My sincere thanks to Mr. Y. C. Feng, Dr. D. Rogers, Dr. Y. Shen, Dr. J. P. Simmons and Dr. B. Wong, with all of whom I have enjoyed extraordinary colleagueship. To Ms. M. Cain and Ms. M. A. Zenchak, I also 
would like to express my warm appreciation for their care and encouragement. I wish to thank Mr. J. Y. Huh, Mr. K. H. Lee and Dr. Y. S. Chung for their support in many aspects and for sharing cherishable experiences. I am obliged to express my wholehearted gratitude to Dr. T. M. Cho, Ms. M. Baco and Mr. H. Baxi, through whom I was able to grow from a Selfblind Platonic caveman who is yet to be reminded of his peripheral existence.

Finally, I would like to express my deepest gratitude to my family for their trust and love. To my parents who have been the fountain of who I am, I dedicate this thesis. 


\section{Table of Contents}

1. Introduction. 1

1.1. $L 1_{0}$ ordering $(f c c \rightarrow f C)$.

1.2. Bain Transformation ( $f c c \rightarrow b c c$ ) during Decomposition of Off-stoichiometric 7 Ordering Alloys.

2. Theoretlcal Background.

2.1. Mean Field Theories of Order-Disorder Transformation. 13

2.1.1. The Phenomenological Landau-Lifshitz Theory. 14

2.1.1.1. Landau-Lifshitz Free Energy and Symmetry Rules. $\quad 14$

2.1.1.2. Second Order Order-Disorder Transformation. 16

2.1.1.3. First Order Order-Disorder Transformation I. 17

$\begin{array}{ll}\text { 2.1.1.4. First Order Order-Disorder Transformation II. } & 19\end{array}$

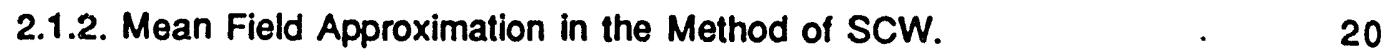

2.2. Continum Elastic Theory of a Plate-like Precipitate. 24

2.2.1. Strain Energy of a Coherent Plate-like Precipitate. 25

2.2.2. Habit Plane of a Plate-like Precipitate. 26

2.2.3. Stability of a Habit Plane. 28

2.2.4. Crystal Geometry of a Constrained Plate-like Precipitate. 29

2.2.5. Shape of a Plate-like Precipitate. 31

3. Thermodynamic Consideration of the Tetragonal Lattice Dsitortion 36 of the L10 Phase.

3.1. General Formulation of Incoherent Two Phase Equilibrium.

3.2. Free Energies $\left(f_{\text {inooh }}\right)$ of $<001>i \infty c$ Special Point Ordered Structures. 41

3.2.1. Formulation of $F_{\text {rigide }}=0$. 41

3.2.2. Formulation of $E_{1}+E_{2}$. 42

3.2.3. Formulation of $E_{3}(\eta)$. 43

3.2.4. Configurational Free Energies $(\theta)$ Stress Free States. 44

3.3. Results and Discussion. 45

3.3.1. Long Range Order Parameter of the Stress Free $L 1_{0}$ Phase. 46

3.3.1.1. $\tau_{i}, \tau_{i}^{+}$and $\tau_{0} . \quad 48$

3.3.1.2. Threshold $\alpha\left(=e / V\left(k_{0}\right) \|\right)$ Value. $\quad 50$

3.3.2. Two Phase $\left(f c c+L 1_{0}\right)$ Equilibrium.

3.3.3. Phase Diagram of the $<001>10 c$ Special Point Ordered Structures. $\quad 56$

3.4. Conclusion. $\quad 62$

4. Effects of Tetragonal Distortion on the Morphology of L1. Ordered 64 Microstructure. 
4.1. Experimental Procedure. $\quad 64$

4.2. Experimental Results. 65

4.2.1. As-Quenched State. $\quad 65$

4.2.2. Aged States. 68

4.2.2.1. Development of Microstructure and Variant Volume Fraction. $\quad 68$

4.2.2.2. The Habit Plane and the Shape of a Variant Plate. 75

4.3. Discussion.

$\begin{array}{ll}\text { 4.3.1. Variant Habit Plane. } & 80\end{array}$

4.3.2. Variant Volume Fraction. $\quad 82$

$\begin{array}{ll}\text { 4.3.2. Shape of a Variant Plate. } & 87\end{array}$

$\begin{array}{ll}\text { 1.4. Conclusion. } & 88\end{array}$

5. Baln Transformation during Decomposition of a hypereutectold 91 Cu-Be alloy.

5.1. Experimental Procedure. $\quad 92$

5.2. Experimental Results. $\quad 92$

5.2.1. Bain Strain Relaxation in As-quenched States. 92

5.2.1.1. Overall Characteristics of a Rapidly-Quenched State. 92

5.2.1.2. Polydomain Precipitate Plates in As-quenched States. 98

5.2.1.3. High Resolution TEM (HRTEM) Study of a Rapidly-Quenched 107 State.

5.2.2. Bain Strain Relaxation Toward Equilibrium. $\quad 114$

$\begin{array}{ll}\text { 5.2.2.1. Air-Cooled State. } & 114\end{array}$

$\begin{array}{ll}\text { 5.2.2.2. Quenched/Aged State. } & 119\end{array}$

$\begin{array}{ll}\text { 5.3. Discussion. } & 122 \\ & 1192\end{array}$

5.3.1. Eartiest Stages Decomposition; Stages of Passive Relaxation of the 122 Bain Strain.

5.3.2. Rain Strain Relaxation during Post- $\alpha^{\prime}$ Stages. 127

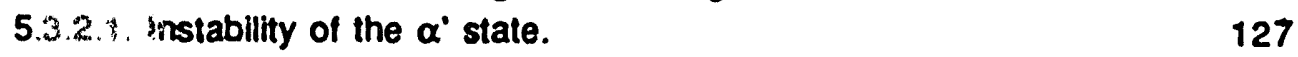

5.3.2.2. Habit Rotation of a Polytwin Plate and Comparison with a 130

Single Domain Plate Case.

5.3.2.3. Formation Mechanism of the Saw-tooth Type Polytwin Plates. $\quad 134$

5.4. Conclusion.

6. Summary. 142

$\begin{array}{lr}\text { References. } & 146\end{array}$

$\begin{array}{ll}\text { Appendix. } & 151\end{array}$ 
Figure 1.1: Schematic diagram depicting the change in crystal structure due to ordering of the disordered foc to the fully ordered L1, phase in an equiatomic binary alloy. Notice tetragonal distortion as well as change in atomic configuration in the $L 1$, ordered structure. A continuum (wave) description of the occupation probabilities $p(r)$ of the solute atoms is also presented for each crystal structure.

Flgure 1.2: The Fe-Pd binary phase diagram (taken from [29]). Metastable (fcc $\left.+L 1_{0}\right)$ two phase field boundaries are schematically superimposed in dotted lines.

Flgure 1.3: The Cu-Be binary phase diagrams (taken from [36]). (a) The entire phase diagram. (b) A high temperature section in the hypereutectoid composition range.

Figure 1.4: Schematic diagram illustrating the crystal structures of the phases involved in the decomposition reaction; $\beta(b c c) \rightarrow \gamma(B 2)+\alpha(f c c)$. Notice that the decomposition reaction involves bcc to $\mathrm{B} 2$ ordering. composition separation and the bcc to fcc Bain crystal lattice reconstruction.

Figure 2.1: Variation in the Landau free energy change $\left(F(\eta)-F_{0}(\eta=0)\right)$ with temperature in order-disorder transformation of (a) second order (b) first order I (c) first order II. See text.

Figure 2.2: A contour $y=y(x)$ describing the shape of a plate-like precipitate on the habit plane (taken from [24,62]). See text.

Flgure 3.1: Schematic diagram illustrating renormalization of rigid lattice free energies into stress free lattice equivalents.

Figure 3.2: Temperature ( $\tau)$ dependence of the long range order parameter $(\eta)$ for various $\alpha$ values. When the tetragonal distortion is suppressed $(\alpha=0)$, the $L 1_{0}$ ordering transition is predicted to be thermodynamically of second order (A). As $\alpha$ increases, the ordering transition changes its character to weakly first order (B) and to strongly first order (C). This indicates that the problem of a rigid lattice model becomes increasingly serious as $\alpha$ value of an $L 1_{0}$ ordering alloy becomes larger.

Figure 3.3: (a) Temperature ( $\tau$ ) dependence of the long range order parameter $(\eta)$ for $c=0.5$ and $\alpha=0.1$. (b) Associated free energy diagrams ( $\Delta f^{\prime}$

vs. $\eta)$ for three different characteristic temperatures. 
Figure 3.4: Calculated phase diagrams for various $\alpha$ and $\omega$ values. No $L_{1}$ phase is allowed in these phase diagrams. (a) $\omega=1$. (b) $\alpha=0.05$. From (a), notice that, consistent with the results shown in Figure 3.2, phase diagram characteristics change drastically as $\alpha$ increases from $\alpha=0$ (rigid state).

Figure 3.5: (a) Phase diagram $C(\alpha=0.05$ and $\omega=1)$ of Figure 3.4 with additional thermodynamic information. Under the diminishing influence of the strain energy term, lines of three characteristic temperatures merge into a single line at $\tau=\tau_{A}$ and two phase boundaries merge into a second order transition line at $\tau=\tau_{B}$. (b,c and d) Associated free energy-composition diagrams for the three specified temperatures.

Figure 3.5: cont. When $\tau<\tau_{A}$, fcc phase is unstable in the composition range where the free energy of fcc phase is larger than that of $L 1_{0}$ phase. This is shown in dotted lines in (c) and (d).

Flgure 3.6: ' Calculated phase diagrams of $<001\rangle^{*}$ special point ordered structures: (a) with no lattice relaxation and (b) with relaxation of configuration-dependent elastic energy contributions.

Flgure 3.7: Experimental phase diagrams of the CuAu and the InMg alloy systems: taken from [75] and [76], respectively.

Flgure 3.8: The CuAu-CuAu 3 side of the CuAu phase diagram calculated by the mean field theory incorporating the tetragonal lattice distortion of the $L 1_{0}$ phase.

Flgure 4.1: The as-quenched state of an Fe-45at.\%Pd alloy. (a) [100] zone axis SADP. (b) Two-beam BF image: $g=020$. (c) [110] zone axis SADP. (d) Two-beam BF image: $g=220$. Notice a very faint intensity of the superfattice reflections, suggesting the existence of the short range order clusters of the $\mathrm{L}_{1}$ phase. This, logether with a high elastic anisotropy of the Fe-Pd alloy, is responsible for the fairly strong diffuse streaks in (a) and (c) and the noticeable mottled (yet faint tweed) strain contrast in (b) and (d).

Figure 4.2: Alloy aged at $450^{\circ} \mathrm{C}$ for $2 \mathrm{~min}$. (a) [110] zone axis SADP. (b) Twobeam BF image taken with $\mathbf{g}=002$. (c) SDF image taken with $\mathbf{g}=$ 001. Along with the detectable, yet diffuse intensity of the superlattice reflections in (a), notice from (c) small ordered domains which are more or less randomly distributed throughout the matrix.

Figure 4.3: Alloy aged at $450^{\circ} \mathrm{C}$ for $5 \mathrm{~min}$. (a) [100] zone axis GADP. (b) Twobeam BF image taken with $g=002$. (c) SDF image taken with $g=$ 001. In conjunction with the <011 $\rangle^{\circ}$ streaks in (a) and the pronounced tweed contrast in (b), notice from (c) regular arrays of the ordered domains along the <011> directions. This alignment of particles invo.ves a change in relative volume fraction among different crystallographic variants, as shown by the non-uniform distribution of the intensity of superlattice reflections in (a). 
Figure 4.4: A single $L 1_{0}$ phase microstructure taken from an alloy aged at 450 ${ }^{\circ} \mathrm{C}$ for $48 \mathrm{hrs}$ : it is imaged by the superlattice reflection indicated by an arrow in the SADP. The superlattice intensity denoted as $A$ is presumably due to double diffraction.

Figure 4.5: A single $L 1_{0}$ phase microstructure taken from an alloy aged at 450 ${ }^{\circ} \mathrm{C}$ for 60 hrs: it is imaged by the superlattice reflection indicated by an arrow in the SADP. Notice the well-developed <011> network consisting of altemating regions of two variants. The superlattice intensity denoted as $A$ is presumably due to double diffraction.

Figure 4.6: A single $L 1_{0}$ phase state of an alloy aged at $450^{\circ} \mathrm{C}$ for $48 \mathrm{hrs}$ and subsequently at $480^{\circ} \mathrm{C}$ for $19 \mathrm{hrs}$. (a) [111] zone axis SADP. (b) BF image. (c) SDF image taken with the superlattice reflection indicated by an arrow. The presence of small plate-like regions of the minor variant along with the serrated boundaries between two different variants suggest that a transition from a two variant state to a mono-variant state is taking place.

Figure 4.7: Single L1, phase microstructure taken along the [110] zone axis from an alloy aged at $450^{\circ} \mathrm{C}$ for $48 \mathrm{hrs}$. (a) [110] zone axis SADP. (b) Schematic diagram illustrating the orientations of the variants involved with respect to the foil orientation. (c) BF image. From (c), notice the loop contrast which delineates the shape of a variant plate on its (110) habit plane.

Figure 4.8: Alloy aged at $450^{\circ} \mathrm{C}$ for $96 \mathrm{hrs}$, as observed along the foil normal of [100]. (a) SADP. Superlattice reflections from different variants are denoted as $A$ and $C ; B$ is presumably due to double diffraction. (b) Schematic diagram showing the orientations of the variants involved with respect to the foil normal (direction of observation). (c) SDF image taken with the superlattice reflection denoted as $\mathbf{C}$.

Figure 4.9: Schematic illustrating the origin of the $[001]^{*}$ streaks in the SADP shown in Fig. 4.8a). These streaks are due to the intersection of [101] streaks resulting from the (101) plates with the Ewald sphere at the [100] foil orientation.

Figure 4.10: Schematic illustrating the polytwin complex stipulated in the theories of polytwin morphology. $n$ denotes the normal of the habit plane of a complex plate with a parent phase matrix and $m$ represents the normal of the habit plane of a variant 1 domain with a neighboring domain of the variant 2 .

Figure 4.11: Schematic illustrating the origin of buckling of thin specimens of $L_{0}$ ordered alloys. Arrows as well as circles denote three different crystallographic variants. The non-uniformity in volume fraction of different variants is represented arbitrarily by relative magnitude of symbols in each colony enclosed by dotted lines. 
Figure 4.12: Calculated shapes of a plate-like region of the variant 1 (with $c$ axis parallel to the [100] direction) coherently embedded in a region of the variant 2 (with $\mathrm{C}$ axis parallel to the [010] direction). Compare these with the observed shape shown in Fig. 4.7c).

Figure 5.1: Uverall characteristics of the rapidly-quenched state. (a) SADP taken near the $[100]_{\gamma}$ zone axis. (b) Associated BF image. (c) DF image taken with the precipitate reflection marked 1 in the SADP. (d) SDF image taken with the matrix reflection marked 2 in the SADP. Notice the arrays of very thin precipitate plates with habit plane normals nearly parallel to [010] and $[001]_{\gamma}$ directions. Notice also the lack of APB contrast from (c) and the striation contrast inside the precipitate plates from (b) and (d).

Figure 5.2: (a) [100] zone axis SADP. (b) Schematic illustration of the basic pattem of the SADP ([100] // [100 $\left.]_{\alpha^{\prime}}\right)$. (c) BF image associated with (a). Two different kinds of the striation contrast are marked 1 and 2 in the BF image.

Figure 5.3: Nicrostructure of the Q2 alloy. (a) BF image. (b) DF image taken with the precipitate reflection $\left(g_{1}\right)$ indicated in the associated SADP. As compared to the case of Q1 alloys (Figure 5.1 and Figure 5.2), precipitate plates are noticeably coarser. Majority of precipitate plates display a characteristic saw-tooth morphology which is expected of polytwin plates.

Figure 5.4: (a) SADP taken from the same region of the Q2 alloy shown in Fig. 5.3 along the $[100]_{\text {z }}$ zone axis. (b) Schematic illustrating the constitution of the SADP.

Figure 5.4: cont. (c) Schematic illustrating the origins of the two fold-split distinct precipitate reflections and of the streak-like intensity. Each of these features is marked by arrows in (a).

Flgure 5.5: Comparison between two different kinds of streak-like intensity. (a) Enlargement of a section in Fig. 5.2a). The streak-like intensity is due to stacking faults. (b) Enlargement of a section in Fig. 5.4a). The streak-like intensity is due to thin twin domains. Nofice that the streak-like intensity in a) differs in both position and direction from the one in b). Compare a) and b) with Fig. 5.6 and Fig. 5.4c), respectively.

Figure 5.6: Schematic illustrating the streak-like intensity due to stacking faults in Fig. 5.2a) and in Fig. 5.5a).

Figure 5.7: TEM micrographs taken from the Q1 as-quenched state by use of the same diffraction condition as that of Fig 5.3b). (a) BF image. (b) DF image. Notice that thin twin domains are now virtually nonexistant in the plates of the varaint 3.2. A few of the detectable thin twin domains are marked by arrows in $\mathrm{h!}$. 
Flgure 5.8: Many beam lattice image taken along $[100]_{\gamma} / /[110]_{\text {prec }}$. Notice a strong correlation between the intersection of plates and the formation of planar defects.

Figure 5.9: Atomic resolution image of the region marked $A$ in Fig. 5.8. Notice that near the defects marked by arrows (whose nature is not exactly known), precipitate unit cells are not only more elongated along the thickness direction but also rotated relative to the unit cells away from defects.

Figure 5.10: Atomic resolution images of the planar defects marked by $B$ and $C$ in Fig. 5.8. (a) $B$; intrinsic stacking fault. (b) $C$; extrinsic stacking fault. Compare with Fig. 5.11.

Figure 5.10: cont. (c) Atomic resolution image of the planar defect marked by $D$ in Fig. 5.8. Notice a thin twin domain of unit cell width. Compare with Fig. 5.11 .

Figure 5.11: Schematic illustrating the (111) stacking sequence in various planar defects. Notice that the number of twinned layers in each type of planar defect is (a) 2, (b) 3 and (c) minimum 4. 1 denotes the mirror plane of a local stacking sequence.

Figure 5.12: TEM photographs showing the main characteristics of the air-cooled state. (a) BF image. (b) Associated SADP taken along the [100] $/$ " $[110]_{\text {prec }}$ zone axis. (c) DF image taken with the precipitate reflection indicated in (b). A region of variant 3.1 and that of variant $3.1^{T}$ are marked by arrows in (c).

Figure 5.13: Schematic illustrating the changes in SADP with progress of the segment-wise habit rotation of the saw-tooth type polytwin plates. Notice the gradual diminishing of the angle separation between the precipitate reflections of $002\left(3.1^{\mathrm{T}}\right)$ and $002(2 \cdot 1)$

Figure 5.14: TEM photographs taken from an alloy aged at $300^{\circ} \mathrm{C}$ and for 24 hrs. (a) BF image. (b) Associated SADP. (c) DF image taken with the 002 precipitate reflection indicated in (b). Notice, in contrast to the saw-tooth morphology of the air-cooled alloys, individual precipitate plates with each having a respective habit plane globally rotated over the whole plate.

Figure 5.15: TEM pho'. graphs taken from an alloy aged at $300^{\circ} \mathrm{C}$ and for 24 hrs. (a) and (c) BF images. (b) and (d) Associated SADP's. Notice a characteristic spatial distribution of the precipitate plates forming a macroscopic polytwin configuration.

FIgure 5.16: (a) Schematic of the CuBe phase diagram. See text. (b) Chemical free energy vs. composition diagram consistent with the phase diagram characteristics at an arbitrary temperature $T_{1}$. When supercooley to $T_{1}$, a disordered $\beta$ phase of the composition $c^{\beta}$, would decompose along the path $A \rightarrow B \rightarrow C \rightarrow D$ to produce the equilibrium foc precipitates. 
Figure 5.17: An octant of the elastic energy surface as a function of habit plane normals of a fcc plate-like precipitate embedded in the B2 matrix. Notice the local maximum along the [001] direction, indicating that the $\alpha^{\prime}$ state is elastically unstable.

Figure 5.18: Calculated elastic strain energy of a polytwin plate consisting of variants 2 and 3 for different habit plane normals of the [hOK] type. Notice that the strain energy of a polytwin plate is lower than that of a single domain plate for the entire range of habit plane normals and that it completely vanishes at a particular $\theta$ value: $\theta=23.56^{\circ}$.

Figure 5.19: Calculated elastic strain energy of a polytwin plate consisting of Vuriants 2 and 3 for different habit plane normals of the [Ohk] type. Notice that the strain energy of a polytwin plate gradually approaches that of a single domain plate until the energy difference completely vanishes at the strain energy minimum of a single domain plate.

Figure 5.20: Schemafic illustratling the generation of a quasi-misfit dislocation as a consequence of impingement of a variant 2 plate (upper crystal) with the broad face of a variant 3 plate (lower crystal). The dislocation dissociates to produce a mobile Shockley partial which then glides to greate an intrinsic gtacking fault on the (111) plane. 


\section{List of Tables}

Table 5.1: Comparison between the $Q 2$ and the air-cooled state in terms of the Bain strain relaxation. The definition of each measured parameter is given in the associated schematic diagram. 


\section{Introduction}

Phase transformations in solids are, as a rule, accompanied by reconstruction (or rearrangement) of the crystal lattices. The reconstruction of the crystal lattice of a parent phase into a new phase lattice involves a lattice deformation which, in general, consists of homogeneous and inhomogeneous distortions (e.g. shuffles). Of these, the homogeneous distortion causes macroscopic effects which are related to the stability and the morphology of a transformed product. It, therefore, constitutes a parameter of great significance in discussions of the thermodynamics of phase transformations which involve crystal lattice reconstructions. A phase transformation for which maoroscopic effects can be treated by a homogeneous distortion alone, is called displacive phase transformation.

In diffusional phase transformations, a crystal lattice reconstruction may occur either as a consequence of or in conjunction with the atomic redistribution processes such as atomic ordering and clustering. In either case, homogeneous lattice distortion accompanying the crystal lattice reconstruction is unavoidably linked to the changes caused by diffusional atomic reconfiguration processes. In the present thesis, we study two selected phase transformations of this class in ordering alloys, in regard to the thermodynamic stability and the morphologies of transformed products. The transformations of our interest are the $L_{0}$ ordering and the Bain transformation which occurrs in off-stoichiometric B2 ordering alloys. These involve fcc to fct and $b c c$ to fcc crystal lattice reconstruction, respectively. 


\subsection{L1。 Ordering $(f c c \rightarrow f c t)$}

The crystal structure of the completely ordered $L 1_{0}$ phase is schematically depicted in Fig. 1.1 along with that of the disordered fcc phase. In terms of atomic configuration, the structure is characterized by the alternation of A and B atom layers on successive (002) planes. It should be emphasized that in association with the characteristic alternate layering, the structure is homogeneously distorted from the $r c c$ along its tetragonal axis i.e. along the direction of layering. Numerous experimental studies have been conducted to show thai the tetragonal lattice distortion of the L1 $1_{0}$ phase depends on the state of order of the atomic configuration. Among many others, an early $X$-ray investigation made by Roberts [1], in particular, presents this point specifically. By correlating the tetragonality values with long range order parameters, measured from the equiatomic CuAu alloys annealed at various temperatures, it was shown that the tetragonal distortion of the $L \mathcal{1}_{0}$ ordered structure is a thermodynamic quantity which can be directly related to the quadratic of the long range order parameter. A question immediately posed by such a link between the atomic configuration and the lattice geometry of the $L 1_{0}$ phase is; what consequences may it lead to either in the thermodynamics of the $\mathrm{LI}_{0}$ ordering, in the alloy phase equilibria involving the $\mathrm{LI}_{0}$ phase, or in the morphology of the $\mathrm{LI}_{0}$ phase. To explore these questions is considered to be essential for a better understanding of the $\mathrm{LI}_{0}$ ordering transformation and this is the main objective of the first part of the thesis.

In respect to theory, an understanding of the fcc to $\mathrm{L1}_{0}$ atomic ordering (more extensively, ordering in the fcc lattice) has been a long standing problem. Most of the present theoretical perspectives of such transformations were put forward through various evaluation methods of the Ising model and of its extensions in terms of the range of atomic interaction. According to these models, the configuration-dependent part of the energy of an alloy is approximated by the sum of pairwise interatomic interactions while atoms are assumed to sit on the lattice sites of a non-deformable rigid lattice. An initial effort to evaluate 


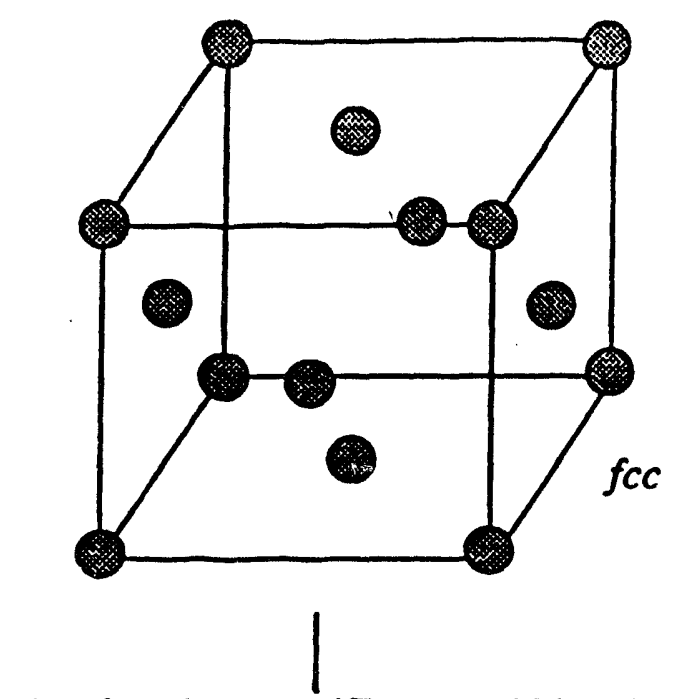

Atomic ordering and Tetragonal Distortion
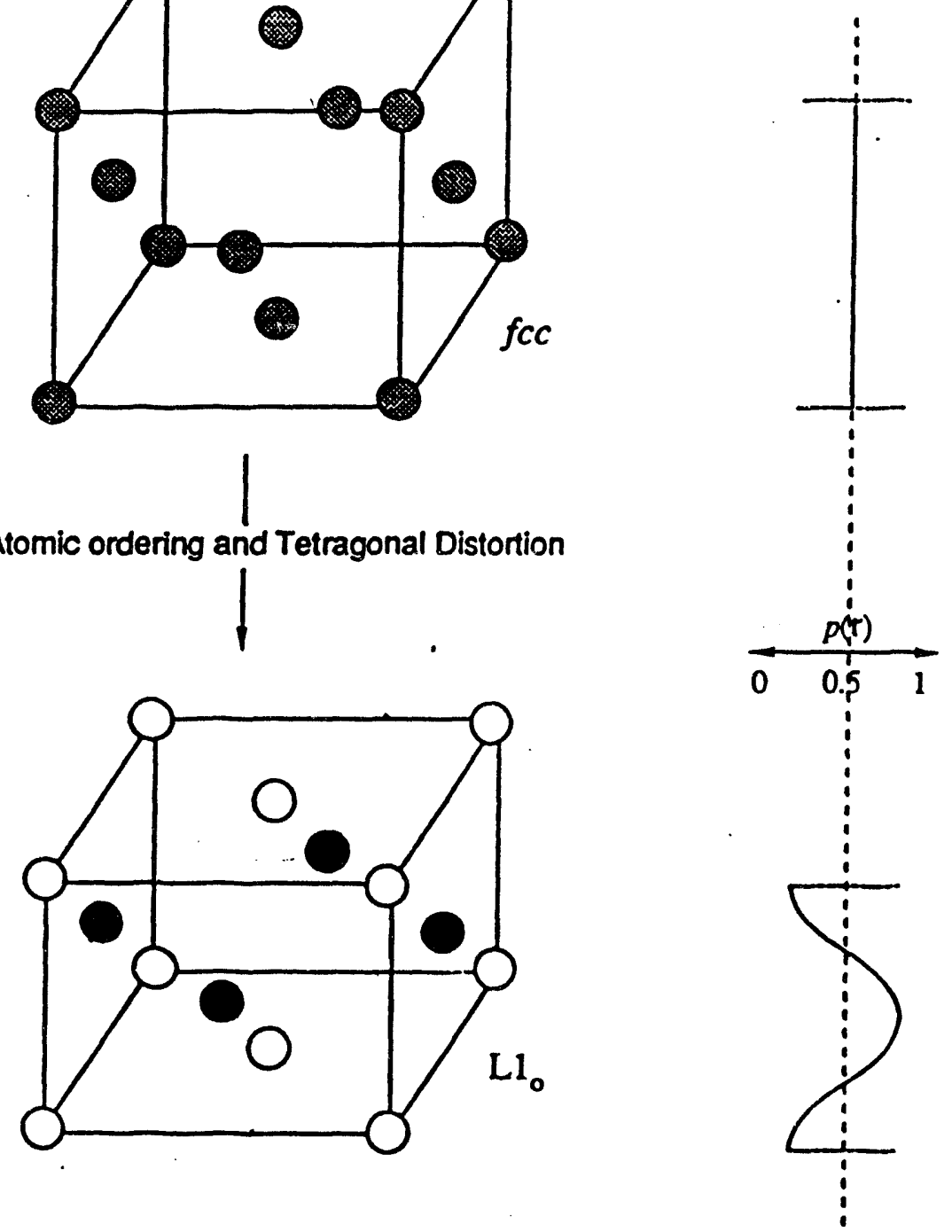

Figure 1.1: Schematic diagram depicting the change in crystal structure due to ordering of the disordered foc to the fully ordered $L 1_{0}$ phase in an equiatomic binary alloy. Notice tetragonal distortion as well as change in atomic configuration in the $L 1_{0}$ ordered structure. A continuum (wave) description of the occupation probabilities $p(r)$ of the solute atoms is also presented for each crystal structure. 
the rigid lattice Ising model in an application to the $<001>^{\star}$ special point ordering ${ }^{\dagger}$ in the fcc lattice was made by Shockley in his calculation of a phase diagram of the prototype CuAu system by use of the Bragg-Williams approximation ( Mean Field approximation ${ }^{\dagger \dagger}$ ) [2]. The result showed that when the mean field approximation is used, the fcc to $\mathrm{LI}_{0}$ ordering on a rigid lattice is predicted to be thermodynamically of second order. Thus, regions of two phase (fcct $\mathrm{L1}_{0}$ ) equilibrium were not reproduced in his calculated phase diagram. Later, from more elaborate calculations based on the first order approximation [3], Cluster Variation Method [4 71 and Monte Carlo method $[8,9]$, the failure of the mean field approximation was explained to arise from an intrinsic defect, namely, the neglect of short range correlation effects.

A different view was put forward by Tachiki and Teramoto $(T-T)^{[10]}$ and later by Kajitani and Cook $(K-C)$ (11] during their theoretical analyses of the $L 1_{0}$ ordering transformation in the equiatomic CuAu system. By recovering the strong first order nature of the transformation through the incorporation of a tetragonal lattice distortion into the Bragg-Williams approximation to the rigid lattice Ising model, they suggested that the earlier failure of the mean field approximation should be ascribed to the limitation of the rigid lattice model itself, rather than to a defect of the approximation. For the past decade, with the advance in understanding of the fundamental properties of the Ising model, the problem of the rigidity of the model has attracted renewed attention in conjunction with theoretical calculations of incoherent order-disorder phase diagrams [12, 13]. In none of these works, however, has due regard been given to the lattice distortion accompanying the fcc to $L_{0}$ ordering and little understanding has been acquired beyond that of T-T and K-C. We believe that the theoretical perspective proposed in the works of T-T and K-C is of major importance to the understanding of the fCC to L1, ordering and should be developed further to explore

\footnotetext{
I A special point refers to a high-symmetry point in $k$ space, characterized by point group symmetries including intersecting symmetry elements. Al special points, the fourier transform of interatomic potential always has extrema by symmetry requirements alone. Special point ordering occurs when the wave vector of the concentration wave falls onto a special point at which the Fourier transform of interatomic potential becomes minimum.
}

It Although the term is commonly used in a wide sense for any order of approximations which use finite size of clusters, we will take the narrow sense of the term and reserve it for the zeroth order (or point) approximation. 
questions which are beyond the realm of the thermodynamics of the rigid lattice.

In regard to experiments, the fcc to $L 1_{0}$ ordering has drawn a great deal of attention in a quantity of TEM and X-ray investigations (See for example ref. [14] for review). A thrust of these investigations was to understand the mechanism and the microstructures of the L1。 ordering. According to these studies, the fCC to $\mathrm{LI}_{0}$ ordering initiates by nucleation of three crystallographic variants ${ }^{\dagger}$ of the $L 1_{0}$ phase, each of which has its own axis of tetragonal distortion along one of the three different cube directions of the fcc phase. As ordering advances, regions of each variant grow with enhanced tetragonal distortion. This drives the ordering system to develop the microstructures which can effectively relax or accommodate the distortion. 'Distortion and relaxation' was, therefore, suggested to be a crucial aspect to consider in discussions of the mechanisms and the microstructures of the $L_{0}$ ordering. Indeed, certain important microstructural features of the $L 1_{0}$ ordering have been also observed in the fcc to fct martensitic transformation [15 - 19], despite a significant difference in kinetic nature.

Inasmuch as similarity in microstructures is noticeable between two different types of transformations, present understanding of the microstructures of the $\mathrm{LI}_{0}$ ordering is, by large, owed to theoretical perspectives [20 - 23] which derive from the selective treatment of homogeneous lattice distortion. This raises a due question: to what extent, such theoretical perspectlves can be utilized to understand the microstructures of the $\mathrm{LI}_{\mathrm{p}}$ ordering ?. This is believed to be an important question to explore, yet, in our survey, it has rarely been examined systematically in previous experimental works. A complete understanding of the mechanisms and the microstructures of the $L_{0}$ ordering system may need to involve kinetic studięs including simulations which employ a proper free energy model in the framework of microscopic diffusion theory $[24,25]$. In a situation where thermodynamic treatments of the

\footnotetext{
I In the metallurgical literature, the term variant is used in various contexts referring to cystallography, e.g. habit orientation variants, rotational variants eic. In this document, unless specified othenwise, variants are meant to be the ones resulting from the reduction in rotational symmetry of the parent crystal structure during phase transformation.
} 
L1 10 ordering are still far from satistactory, one may want to take a conservative approach and to assess carefully the existing perspectives which complement the other polarized views offered by rigid lattice models.

In summary, current understanding of the fcc to $L_{1}$ ordering is founded on two diametrically contrasting standpoints. Theoretical treatments of the thermodynamics of the L1. ordering have been based on the unrealistic microscopic models which are only concerned about atomic reconfiguration with the neglect of the lattice distortion accompanying the ordering. On the other hand, interpretations of experimental findings have been based on macroscopic treatments of geometrical theories of martensite and elasticity theories which deal with the lattice distortion selectively. The lack of contact between two different line of efforts has, as a result, failed to produce an integrated understanding of the ordering more than it has furnished a complementary knowledge. It is thus necessary that an effort should be made to initiate studies which will fill the gap of our knowledge resulting from the existing bilateral approaches.

The first part of the thesis is organized as follows. In chapter 3, we present our theoretical investigation of the thermodynamics of the $\mathrm{LI}_{0}$ ordering and of the phase equilibria involving $\mathrm{LI}_{0}$ phase. To lift the rigidity of the Ising model is not an easy task. A complete approach to this problem necessarily involves the use of realistic interatomic potentials derived from first principle considerations. This is, however, beyond the scope of the present work. Instead, we take an indirect approach following the manner of T-T and K-C. The approach basically concerns itself with the release of non-physical forces associated with the volume and the crystal shape constancy condition imposed on the thermodynamic potentials (Helmholtz free energy or grand potential) resulting from various approximations to the rigid lattice Ising model. The advantage of this approach is such that one can treat the problem of lifting the rigidity purely as an elasticity problem and study its consequences independently without exploring more complicated fundamental questions. For the rigid lattice free energy, we use the mean field approximation in the Static Concentration Wave (SCW) method [26-28]. It is 
shown to provide an efficient framework which enables us to discuss qualitatively important thermodynamic characteristics of the $\mathrm{LI}_{0}$ ordering system.

In chapter 4, we present the results of an experimental investigation of the effects of tetragonal lattice distortion on the morphology of the L1。 phase. For this purpose, we use the $\mathrm{Fe}-45$ at.\%Pd alloy. The equilibrium phase diagram of the Fe-Pd binary system [29] is shown in Fig.1.2. In the diagram, dotted lines are superimposed to schematically represent the metastable extensions of $\left(f c c+L 1_{0}\right)$ two phase field boundaries. In the course of our study, it was found that during a phase transformation in the Pd-rich regions of the equilibrium (L1。 $+b c c)$ two phase field, stabiliky of $(C C$ and L1。 phases is governed by the metastable extensions for extended periods of reaction time. By use of the as-quenched $\mathrm{Fe}-45$ at.\%Pd alloy as an initlal state, we carry out a detailed documentation of the various ordered states with a particular emphasis on the single L1, phase state. Based on our experimental findings, we then discuss the essential morphological leatures of the single L1。 phase such as habit plane, volume fraction and shape of a variant plate in light of the theories of polytwin-domain morphology and the elasticity theories of a plate-like precipitate.

\subsection{Bain Transformation (bcc<--->fcc) during Decomposition of Off-stoichiometric Ordering Alloys.}

The cubic to tetragonal lattice distortion which we consider for the $\mathrm{L1}_{0}$ ordering is intrinsic to the configurational ordering process itself and the lattice distortion can thus be directly related to the long range atomic order of the $\mathrm{L}_{0}$ phase. In the second part of the thesis, we study the cubic to tetragonal distortion which is not intrinsic to, but interplays with concomitant atomic redistribution processes. viz. the Bain structural transformation during decomposition of ordering alloys. Being attended by atomic ordering and composition separation, a crystal lattice reconstruction may occur in a gradual manner by utillizing paths provided by the accompanying processes. Since the homogeneous distortion due to a crystal lattice 


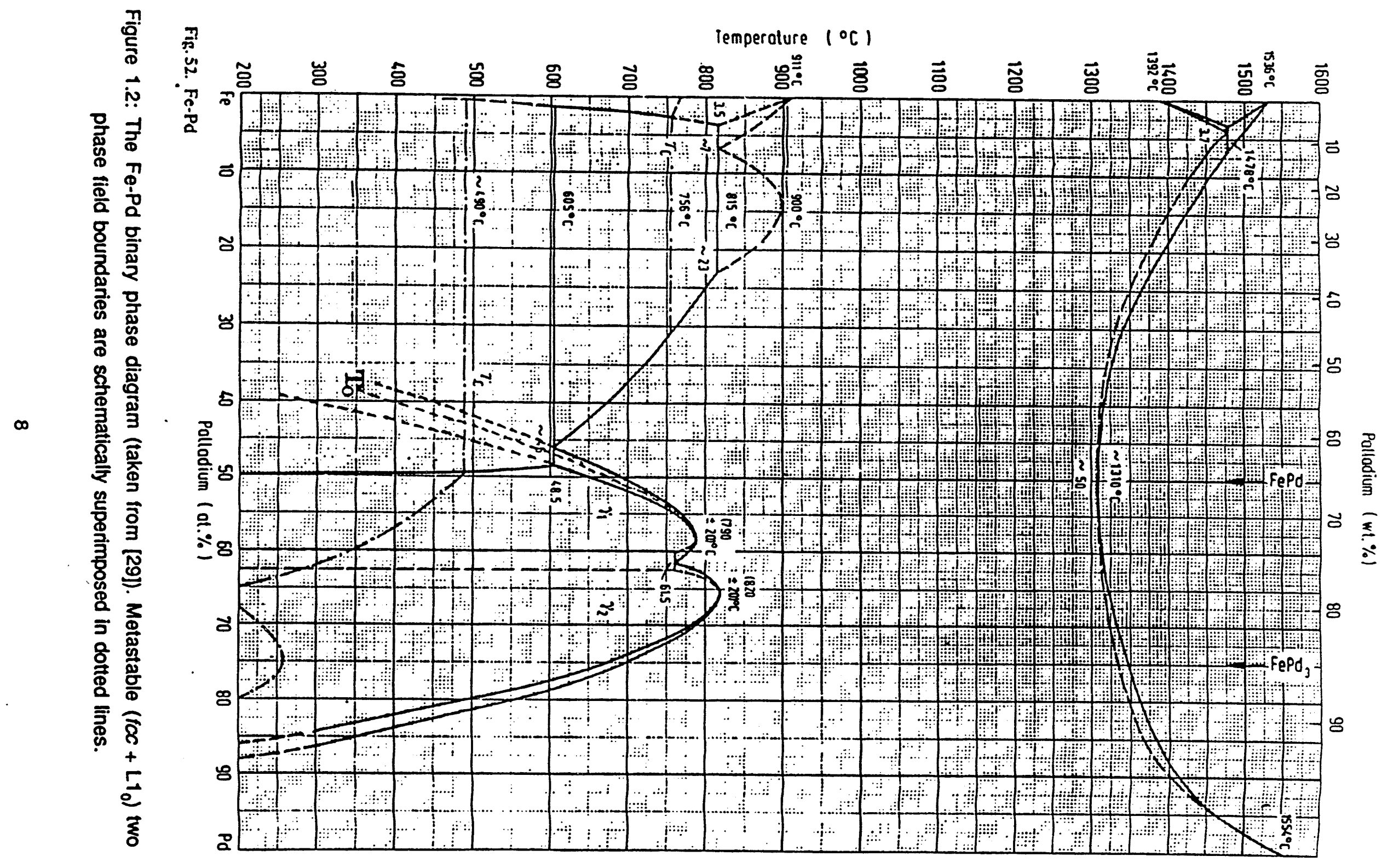


reconstruction plays a determining role in the morphology of a decomposed alloy and a particular morphology of a decomposed alloy depends on the specific path to the equilibrium, it is important to examine and understand the process of a lattice reconstruction in light of possible interactions with attending diffusional processes.

The situation we are considering is not exotic, but has been observed during decomposition of Cu-rich Cu-Be alloys. $\left.{ }^{(30}-33\right]$ According to the experimental works previously made, at large supersaturations, the decomposition proceeds in the following sequence:

$$
\begin{gathered}
\alpha \text { (fcc disordered) } \rightarrow \alpha_{1} \text { (Cu-rich fcc) }+ \text { Be-rich equiaxial G. P. zone } \\
\rightarrow \alpha_{2}+\text { plate-like G. P. zone } \rightarrow \alpha_{3}+\gamma \rightarrow \alpha_{4}+\gamma \rightarrow \alpha_{e q}+\gamma_{\text {eq }} \text { (B2 ordered) }
\end{gathered}
$$

The main focus in this complex decomposition reaction has been placed on the nature of various $\gamma$ phases in conjunction with the fcc to bcc (B2) crystal lattice reconstruction. A consensus made in many experimental studies has been such that $\gamma$ and $\gamma$ phases are metastable phases which have distinct crystal structures from that of the equilibrium $\gamma$ phase. Based on this standpoint, much effort has been devoted to investigating the formation mechanism of various $\gamma$ phases in successive stages of the decomposition. Recently, a new proposal has been made by Khachaturyan and Laughlin (K-L) [34]. By applying the elastic theory developed earlier by Khachaturyan [35], they have enunciated that the different $\gamma$ phases are not distinct individual phases but are the same cubic $\mathrm{B2}$ phase in different elastic constrained states, each of which is characterized by a different habit plane orientation, According to their proposal, the transition from $\gamma^{\prime \prime}$ to $\gamma^{\prime}$ phase is realized along a decomposition path by the diffusional reorientation of the habit plane from $\{001\}$ to the one satisfying elastic equilibrium. The results of their calculations based on this concept have actually shown quite good agreement with the existing experimental data and furthermore, have resolved discrepancies among different experimental interpretations. We believe that the spirit manifested in their proposal is very helpful and may be utilized to extend our understanding of other decompositions of the same class.

The particular alloy system chosen for this part of the investigation is the hyper-eutectold 
Cu-9.04 wt\%Be binary alloy for which overall decomposition reaction is represented as; $\beta$ $(b c c) \rightarrow \gamma(B 2)+\alpha(f c c)$. An equilibrium phase diagram of the CuBe system [36] and the crystal structures of the phases involved in the decomposition reaction, are shown in Fig. 1.3 and Fig. 1.4, respectively. Unlike decomposition of $\alpha(f c c)$ phase, decomposition of $\beta$ (bcc) phase of a hyper-eutectoid composition has not been studied in as great detail and only limited information is available from some early investigations [37-39]. According to the TEM observations made by Tadaki et al. [38] and later by Auvray [39], the relaxation of the Bain strain during decomposition of $\beta$ phase is more complex in nature than in the case of decomposition of $\alpha$ phase. This has led to significant discrepancies between interpretations of different experimental studies. We find that the complexity of the relaxation process is not only due to its interplay with the atomic redistribution processes but also due to the concurrence of different Bain strain relaxation mechanisms, specifically, the habit plane rotation and the formation of polydomains (twin domains and translational domains). In chapter 5, we present the details of our experimental findings and analyze them along the line of reasoning of K-L's proposal. 
-26ues

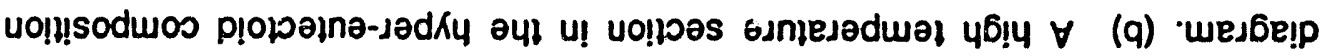

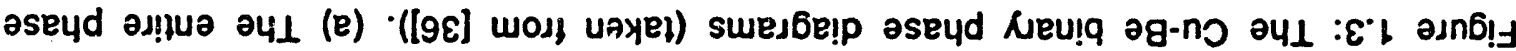

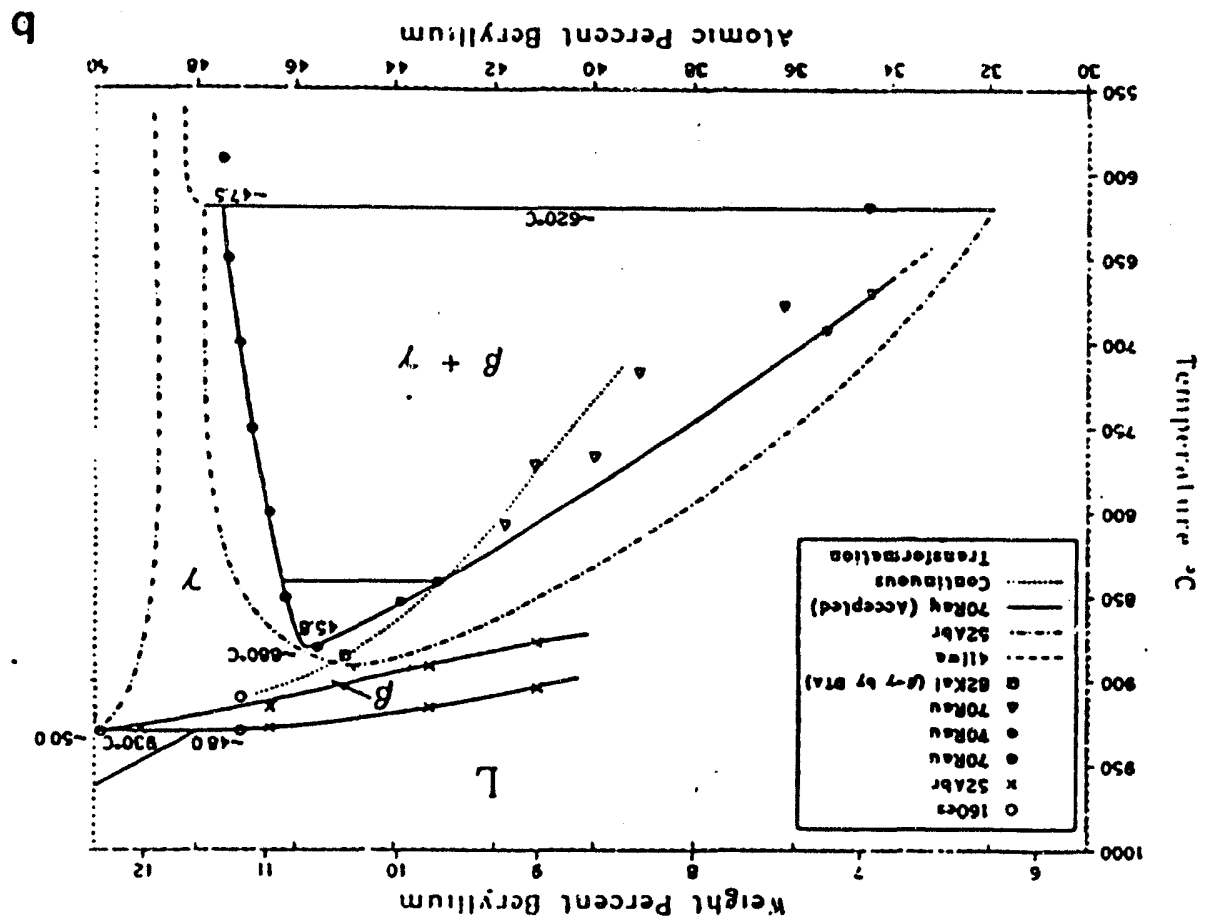

e

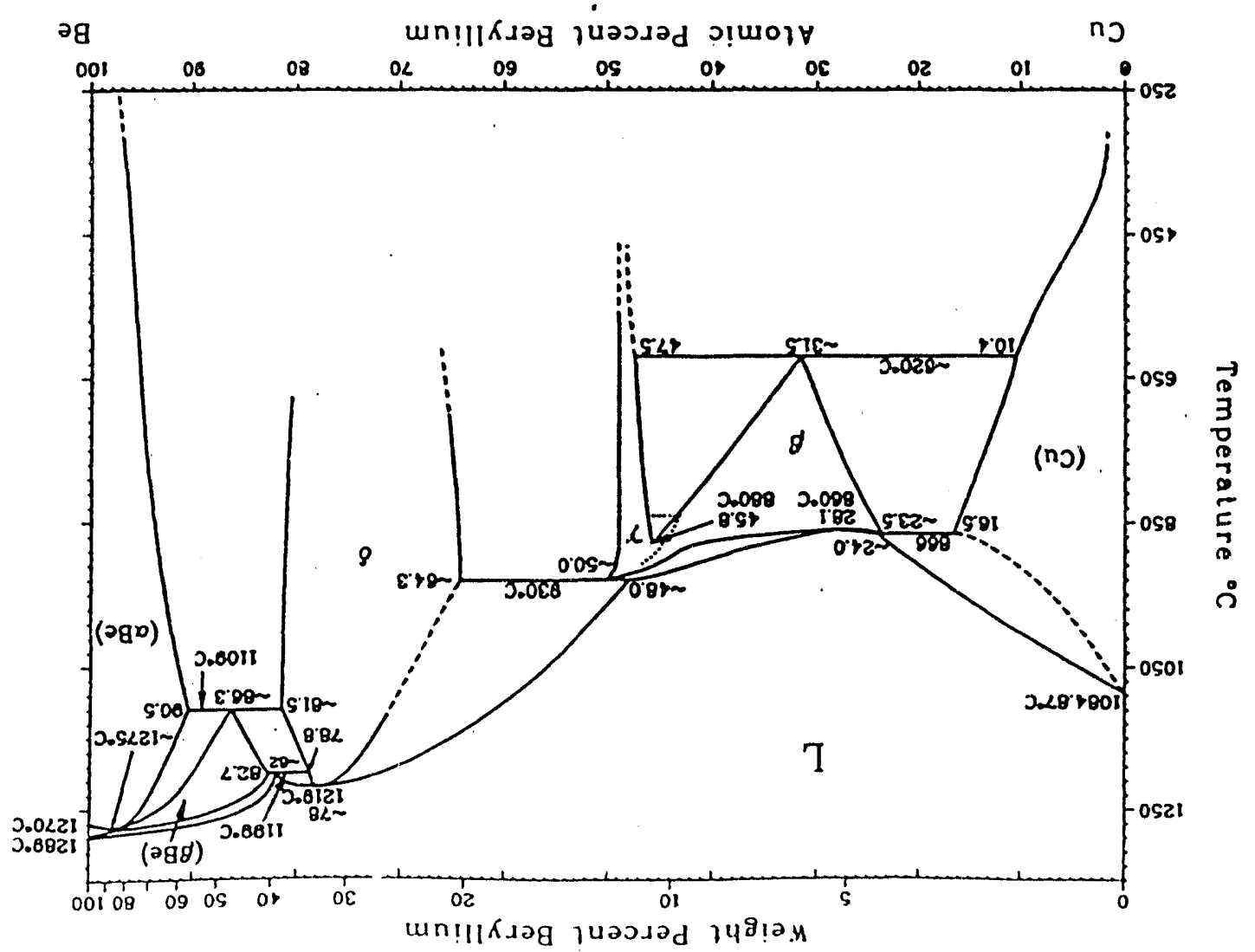



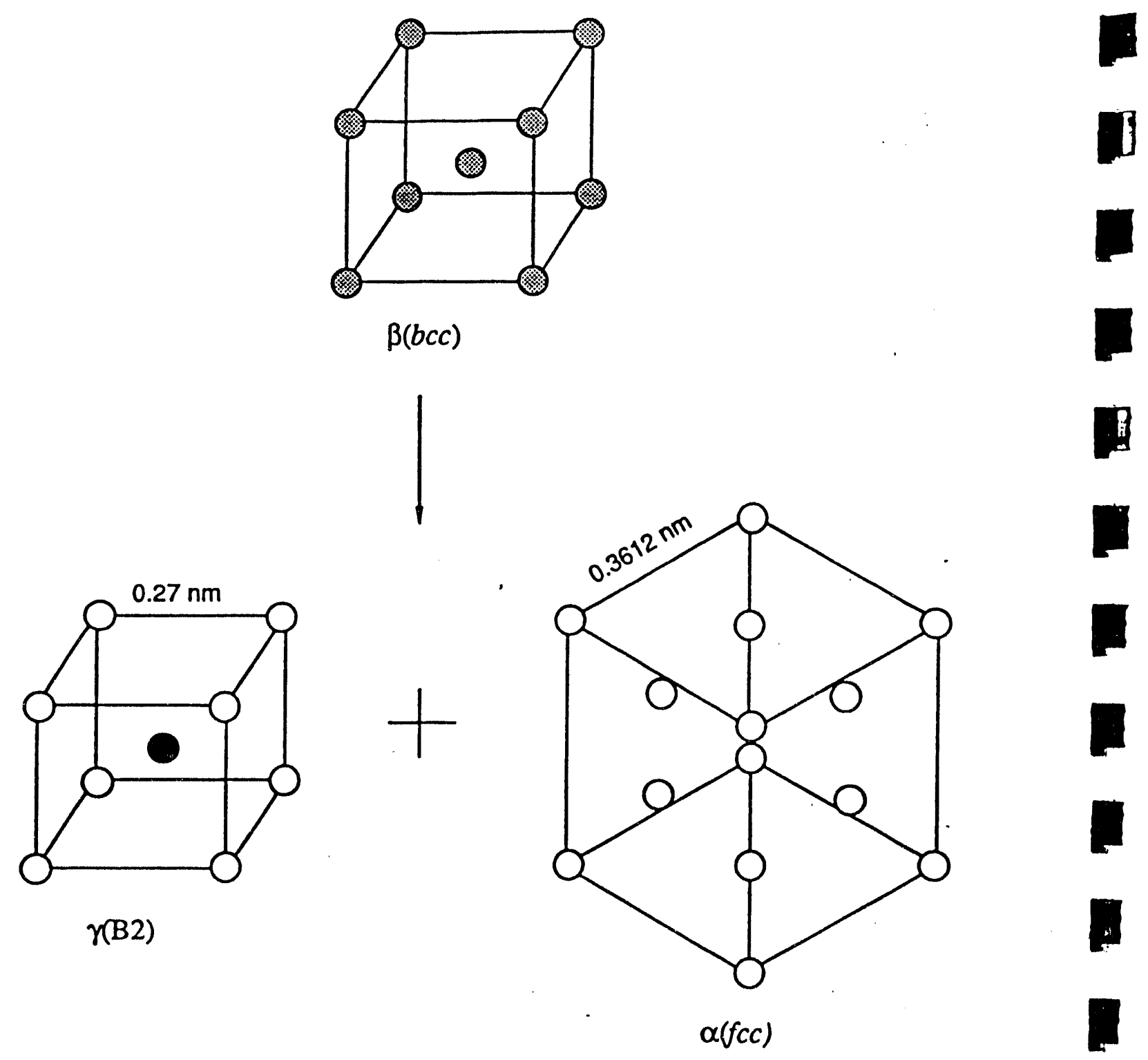

Figure 1.4: Schematic diagram illustrating the crystal structures of the phases involved in the decomposition reaction; $\beta(b c c) \rightarrow \gamma(B 2)+\alpha(f c c)$. Notice that the decomposition reaction involves bcc to $\mathrm{B2}$ ordering, composition separation and the bcc to fcc crystal lattice reconstriction. 


\section{Theoretical Background}

In this chapter, we present important concepts of the thermodynamics of ordering and of continum elasticity, as modeled by the selected theories: mean field theories and continum elasticity theories of a plate-like precipitate. Concepts, formulations and equations introduced here are either further specialized or directly utilized during the discussions in the chapters which follow.

\subsection{Mean Field Theories of Order-Disorder Transformation.}

Mean field theories of the order-disorder transformation in metallic alloys include the phenomenological Landau-Lifshitz theory $(40-44)$ and mean field approximations to the Ising model in statistical mechanics $26,28.45]$. In spite of the simple nature of the theories, it is generally acknowledged that they are sufficiently useful not only to illuminate qualitatively important features of order-disorder transformations but also to provide reliable quantitative predictions in some limited applications (46 48]. In section 2.1.1, we discuss some of the basic predictions of the mean fieid theories by analysis of the more general Landau-Lifshitz's thermodynamic theory. In doing so, we start with describing the k-space formulation of the theory. This will clearly show us inuitful concepts of the theory including symmetry rules governing order-disorder transformations. In section 2.1.2, we then introduce the mean field approximation to the rigid lattice Ising model in the method of SCW. The nature of the mean field approximation and the problems of the resulting rigid lattice free energy will come into view during discussion in this section. 


\subsubsection{The Phenomenological Landau-LIfshltz Theory \\ 2.1.1.1. Landau-LIfshitz Free Energy and Symmetry Rules}

For a binary solid solution of a fixed solute concentration, the average ${ }^{\dagger}$ configurational state of the disordered phase perturbed by concentration fluctuations can be represented by a set of variables $p(r)$ describing the average solute concentration at the lattice site $r$.

$$
p(r)=c+\delta p(r)
$$

where $c$ is the solute concentration averaged over all the lattice sites and $\delta p(r)$ is the mean concentration displacement at the site $r$ : when $\delta p(r)=0$ for every $r$, the solution is disordered. Assuming all the lattice sites can be described by a single Bravais lattice, $\delta p(r)$ may be represented in terms of a family of waves with the wave vectors belonging to the same startt:

$$
\delta p(r)=\frac{1}{2} \sum_{n}\left[Q\left(k_{n}^{0}\right) e^{20}+Q^{*}\left(k_{n}^{0}\right) e-\alpha_{n}^{0}\right]
$$

where $n$ denotes the $n$th component wave of the $\operatorname{star}\left(k_{n}^{0}\right)$. The amplitude $Q\left(k_{n}^{0}\right)$ is defined by

$$
Q\left(k_{n}^{9}\right)=\eta \gamma\left(k_{n}^{0}\right)
$$

where $\eta$ is a long range order parameter and $\gamma\left(k_{n}^{0}\right)$ 's are coefficients determining the point group symmetry of the mean concentration fluctuation (i.e. point group symmetry of the ordered phase ) and can be determined from either the normalization condition $\left.\sum_{n} h_{\gamma}\left(k_{n}^{0}\right)\right|^{2}=1$ or the physical requirement that $p(r)=0$ or 1 for the fully ordered state.

According to the Landau free energy model, the Helmholtz free energy $F$ of the ordered phase with the average atomic distribution given by eq. (2.1) may be represented in a power series of $\delta p(r):(24,49,50)$

$$
\begin{aligned}
F=F_{0}+ & \sum_{r} A_{1}(r) \delta p(r)+\sum_{r, r} A_{2}(r, r) \delta p(r) \delta p\left(r^{\prime}\right)+\sum_{r, r_{1} r^{\prime}} A_{3}\left(r, r_{,} r^{\prime \prime}\right) \delta p(r) \delta p\left(r^{\prime}\right) \delta p\left(r^{\prime \prime}\right) \\
& +\sum_{r, r, r, r} A_{4}\left(r, r_{,} r^{r}, r^{\prime \prime}\right) \delta p(r) \delta p\left(r^{\prime}\right) \delta p\left(r^{\prime \prime}\right) \delta p\left(r^{\prime \prime}\right)+\ldots
\end{aligned}
$$

\footnotetext{
t 'Average' means the average in the microcanorical sense.

${ }^{t} t_{A}$ star is a group of non-equivalent wave vectors each of which can be obtained from one another by the operation of all the symmetry elements in the point group symmetry of the disondered phase.
} 
where $F_{0}$ is the free energy of the disordered phase and coefficients $A_{i}$ 's are defined by

$$
\begin{aligned}
& A_{1}(r)=\left(\frac{\partial F}{\partial p(r)}\right)_{p(r)=c}, \quad A_{2}\left(r, r^{\prime}\right)=\frac{1}{2}\left(\frac{\partial^{2} F}{\partial p(r) \partial p\left(r^{\prime}\right)}\right)_{p(r)=c} \\
& A_{3}\left(r, r^{\prime}, r^{\prime \prime}\right)=\frac{1}{3 !}\left(\frac{\partial^{3} F}{\partial p(r) \partial p\left(r^{\prime}\right) \partial p\left(r^{\prime \prime}\right)}\right)_{p(r)=c} \\
& A_{4}\left(r, r^{\prime}, r^{\prime}, r^{\prime \prime}\right)=\frac{1}{4 !}\left(\frac{\partial^{4} F}{\partial p(r) \partial p\left(r^{\prime}\right) \partial p\left(r^{\prime \prime}\right) \partial p\left(r^{\prime \prime}\right)}\right)_{p(r)=c}
\end{aligned}
$$

Substituting eq.5' (2.2) and (2.3) into eq. (2.4) and taking account of the fact that $A_{i}(\{r\})^{\prime}$ s are functions of $T$ and $c$ describing the thermodynamic state of the disordered phase lead to

$$
F(T, c, \eta)=F_{0}(T, c)+A_{1}(T, c) \eta+A_{2}(T, c) \eta^{2}+A_{3}(T, c) \eta^{3}+A_{4}(T, c) \eta^{4}+\ldots
$$

where

$$
\begin{aligned}
& A_{1}(T, c)=\sum_{n} A_{1}\left(k_{n}^{0}\right) \gamma\left(k_{n}^{0}\right), \quad A_{2}(T, c)=\frac{N}{2} A_{2}\left(k^{0}\right) \sum_{n}\left|\gamma\left(k_{n}^{0}\right)\right|^{2}=\frac{N}{2} A_{2}\left(k^{\circ}, T, c\right) \\
& A_{3}(T, c)=\frac{1}{3 !} \sum_{n} A_{3}\left(k_{n_{1}}^{\circ}, k_{n_{2}}^{\circ}, k_{n_{3}}^{\circ}\right) \gamma\left(k_{n_{1}}^{\circ}\right) \gamma\left(k_{n_{2}}^{\circ}\right) \gamma\left(k_{n_{3}}^{\circ}\right) \\
& A_{4}(T, c)=\frac{1}{4 !} \sum_{n} A_{4}\left(k_{n_{1}}^{\circ}, k_{n_{2}}^{\circ}, k_{n_{3}}^{\circ}, k_{n_{4}}^{\circ}\right) \gamma\left(k_{n_{1}}^{\circ}\right) \gamma\left(k_{n_{2}}^{\circ}\right) \gamma\left(k_{n_{3}}^{\circ}\right) \gamma\left(k_{n_{4}}^{\circ}\right)
\end{aligned}
$$

and $A_{i}\left(\left\{k^{\circ}\right\}\right)$ 's are the Fourier transforms of the $A_{i}(\{r\})$ 's of eq. (2.4).

Important symmetry rules goveming order-disorder transformations can now be derived from the condition that the free energy should be invariant to any symmetry operation belonging to the space group symmetry of the disordered phase. For example, it should remain invariant under the translation of the atomic distribution $p(r)$ by the lattice period $T$ of the disordered phase i.e. $p(r)-\rightarrow p(r+T)$. When this translation is considered in eq.s' (2.2) and (2.3), each $\gamma$ in the coefficients $A_{i}$ 's of eq. (2.5) transforms via $\gamma\left(k_{n_{i}}^{0}\right) \rightarrow \gamma\left(k_{n_{i}}^{0}\right) e^{i n_{i}^{0} T}$. By inspection of the transformed free energy in light of its invariance, the following symmetry rules can be deduced.

The coefficients of the first order term should be identically zero. This is because, otherwise, the wave vectors of a star would necessarily have the translational symmetry of the disordered phase in $k$-space for the invariance of the free energy, in which case, an 
order-disorder transition can not occur. The coefficient of the harmonic term contains no $\gamma$ dependent term, therefore, always has translational invariance. The symmetry rule of prime importance is attained from the invariance of the third order term i.e. $A_{3}^{\prime}(T, c)=A_{3}(T, c)$ where $A^{\prime}{ }_{3}(T, c)$ is given by

$$
A_{3}^{\prime}(T, c)=\frac{1}{3 !} \sum_{n} A_{3}\left(k_{n_{1}}^{\circ}, k_{n_{2}}^{\circ}, k_{n_{3}}^{\circ}\right) \gamma\left(k_{n_{1}}^{\circ}\right) \gamma\left(k_{n_{2}}^{\circ}\right) \gamma\left(k_{n_{3}}^{\circ}\right) e^{i\left(k_{n_{1}}^{\circ}+k_{m_{2}}^{\circ}+k_{n_{3}}^{\circ}\right) T}
$$

The cases which ensure the invariance of this term may be summarized as follows:

$$
\begin{aligned}
\text { if } k_{n_{1}}^{0}+k_{n_{2}}^{0}+k_{n_{3}}^{0} \neq G, A_{3}\left(k_{n_{1}}^{0}, k_{n_{2}}^{\circ}, k_{n_{3}}^{0}\right)=0 \\
\text { if } k_{n_{1}}^{0}+k_{n_{2}}^{\circ}+k_{n_{3}}^{0}=G, A_{3}\left(k_{n_{1}}^{0}, k_{n_{2}}^{0}, k_{n_{3}}^{0}\right) \neq 0 \text { and } \gamma\left(k_{n_{1}}^{0}\right) \gamma\left(k_{n_{2}}^{0}\right) \gamma\left(k_{n_{3}}^{0}\right) \neq 0 \\
\text { or } A_{3}\left(k_{n_{1}}^{0}, k_{n_{2}}^{0}, k_{n_{3}}^{0}\right) \neq 0 \text { and } \gamma\left(k_{n_{1}}^{0}\right) \gamma\left(k_{n_{2}}^{0}\right) \gamma\left(k_{n_{3}}^{0}\right)=0
\end{aligned}
$$

where $\mathbf{G}$ is a fundamental reciprocal lattice vector of the disordered phase. As it turns out, eq. (2.6a) constitutes a necessary condition that has to be fulfilled by a second order orderdisorder transformation. Namely, a phase transition may be thermodynamically of second order when the translational symmetry of the ordered phase is such that it does not maintain the crystal momentum conservation among any three wave vectors of the same star. In the other cases ruled out by eq. (2.6a), a phase transition is expected to be of first order.

\subsubsection{Second Order Order-Disorder Transformation.}

When eq. (2.6a) is substituted into eq. (2.5) along with the condition that $A_{1}(T, c)=0$, we obtain

$$
F(T, c, \eta)=F_{0}(T, c)+A_{2}(T, c) \eta^{2}+A_{4}(T, c) \eta^{4}
$$

where terms of higher order than $\eta^{4}$ are neglected. In this expression, the coefficient of the fourth order term $\left(A_{4}(T, c)\right)$ is always positive to avoid free energy minima at physically meaningless large $\eta$ values. A simple mathematical analysis will disclose the basic properties of the free energy of eq. (2.5a):

From the condition of equilibrium: $\frac{\partial F}{\partial \eta}=0$. 


$$
\eta=0 \text { or } \eta= \pm\left[\frac{-A_{2}}{2 A_{4}}\right]^{\frac{1}{2}}
$$

From the condition of the limit of stabillity of the disordered phase: $\left.\frac{\partial^{2} F}{\partial \eta^{2}}\right|_{\eta=0}=0$,

$$
A_{2}(T, c)=0
$$

On the basis of eq.s' (2.7a) and (2.7b), one can construct the free energy diagram, as schematically shown in Fig. 2.1a). Above the transition temperature where the disordered phase is stable, the free energy must have a minimum uniquely at $\eta=0$. This leads to the requirement that $A_{2}(T, c)>0$ for $T>T_{c}$ where $T_{c}$ is the transition temperature. Below the transition temperature where the ordered phase is stable, $\eta=0$ no longer remains at the free energy minimum, therefore, $A_{2}(T, c)<0$ must be satisfied. Since $A_{2}(T, c)$ changes its sign through $T=T_{c}$, it must vanish at the transition point itself. Eq (2.7b) thus defines the line of second order order-disorder transformations. From these required properties, the coefficient $A_{2}(T, c)$ can be recast into a form:

$$
A_{2}(T, c) \equiv B(c)\left(T \cdot T_{c}\right) \quad \text { where } B(c)>0
$$

In the second order order-disorder transformation, a change of state occurrs continuously as the transition temperature is approached from either side of it. As clearly illustrated in Fig. 2.1a), this means that the disordered phase can not exist in a metastable state below the transition temperature and, similarly, neither can the ordered phase above the transition temperature. It is emphasized that in terms of tree energy, this is directly related to the fulfillment of the condition of eq. (2.6a). In the presence of a non-vanishing third order coefficient, additional free energy minima could possibly occur at each side of the transition temperature.

\subsubsection{First Order Order-DIsorder Transformation 1.}

When the order-disorder transformation follows the selection rule of eq. (2.6b), the Landau free energy gives

$$
F(T, c, \eta)=F_{0}(T, c)+A_{2}(T, c) \eta^{2}+A_{3}(T, c) \eta^{3}+A_{4}(T, c) \eta^{4}
$$




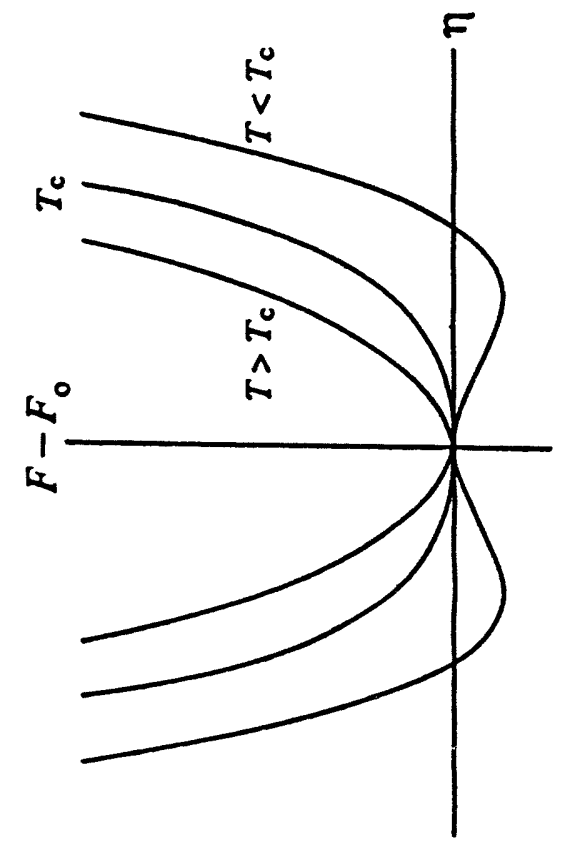

สิ

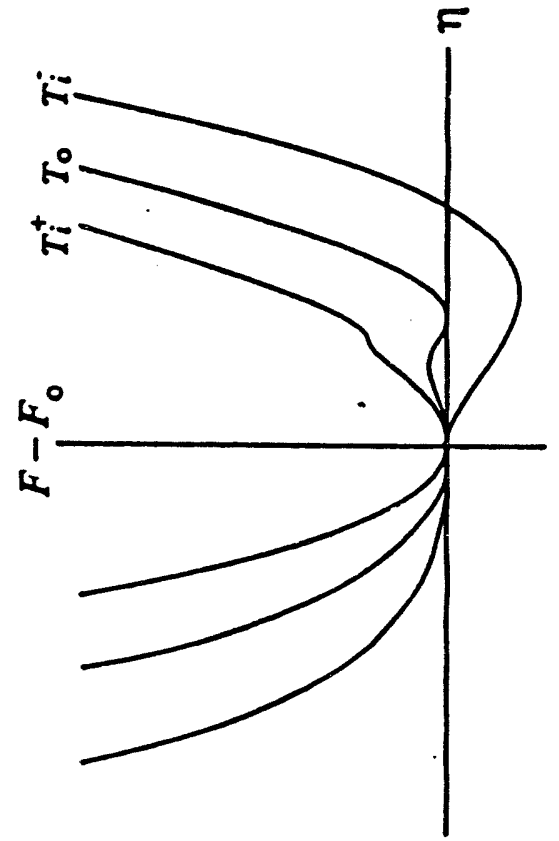

0

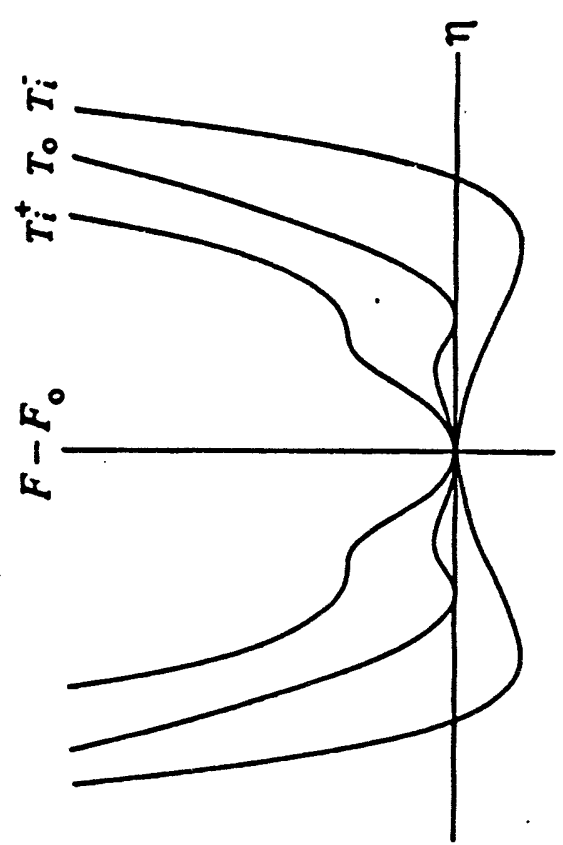

o

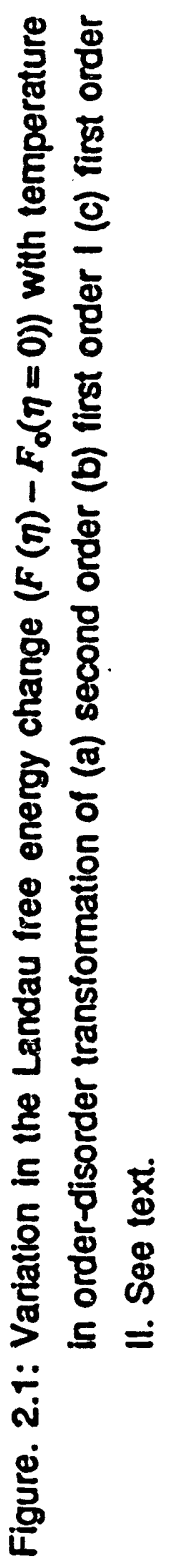


where $A_{4}(T, c)>0$. The same analysis as that of eq.'s $(2.7 \mathrm{a})$ and $(2.7 \mathrm{~b})$ leads to:

From the condition of equilibrium: $\frac{\partial F}{\partial \eta}=0$.

$$
\eta=0 \text { or } 4 A_{4} \eta^{2}+3 A_{3} \eta+2 A_{2}=0
$$

From the condition of the limit of stabillity of the disordered phase: $\left.\frac{\partial^{2} F}{\partial \eta^{2}}\right|_{\eta=0}=0$.

$$
A_{2}(T, c)=0
$$

The, corresponding free energy diagram is shown in Fig. 2.1b). In contrast to Fig. 2.1a), a distinct feature is readily noticed. Over a certain range of temperatures, free energy has a local minimum signifying the existence of a metastable state, in addition to a global minimum for the stable state. The relative thermodynamic stability of one phase to the other in different temperature regimes is entirely characterized by the temperatures shown in the diagram $[43,44,48]$. $T_{i}^{*}$, as defined by eq $(2.9 \mathrm{~b})$, is the temperature at which the disordered phase becomes unstable with respect to ordering (lower instability). At $T=T_{0}$, both phases have the same free energy i.e. $F\left(T_{0}, c, \eta_{e q}\right)=F_{0}\left(T_{0}, c\right)$. Across this temperature, the global minimum of the free energy shifts from one phase to the other. $T_{i}^{+}$is the temperature at which ordered phase becomes unstable with respect to disordering (upper instability). Mathematically, this temperature is defined from the vanishing of the discriminant of the quadratic equation in eq (2.9a). Using the aforementioned definition of each characteristic temperature together with $A_{2}(T, c)=B(c)\left(T-T_{i}^{*}\right)$, the following relations can be derived:

$$
T_{i}^{+}=T_{i}^{-}+\frac{9 A_{3}^{2}}{32 B A_{4}}, T_{0}=T_{i}^{-}+\frac{A_{3}^{2}}{4 B A_{4}}
$$

\subsubsection{FIrst Order Order-Disorder Transformation II.}

The selection rule (2.6c) represents an interesting case where the condition (2.6a) rules out the possibility of the second onder transformation but the free energy tums out to have the form expected of the second order transformation. That is,

$$
F(T, c, \eta)=F_{0}(T, c)+A_{2}(T, c) \eta^{2}+A_{4}(T, c) \eta^{4}+A_{6}(T, c) \eta^{6}+\ldots
$$


where $A_{4}(T, c)>0$. The ordering transformation of the disordered fcc to the ordered $L_{0}$ structure may be a typical example of this case. This ordering transformation has been found to be thermodynamically of the first order. However, Landau theory predicts it to be of the second order due to the fulfilment of the condition $(2.6 \mathrm{c}) .^{\dagger}$

The mathematical form of the free energy which ensures the first order transformation is found to be the one with $A_{4}(T, c)<0$ and $A_{6}(T, c)>0$. Physically, this means that for such order-disorder transitions whose first order nature can be recovered within the framework of the Landau theory, there must exist a negative $\eta^{4}$ contribution overriding the positive one resulting from the reconfiguration due to ordering. Some instances are found in magnetic systems for which the first order nature of magnetic ordering was reproduced by accounting for the negative $\eta^{4}$ contribution due to magnetostriction. ${ }^{51,52]}$ Similarly, the recovery of the first order nature has been attained for the fcc to $\mathrm{LI}_{0}$ ordering in the stoichiometric CuAu alloy by incorporation of the elastic energy resulting from the shape change during ordering. ${ }^{[10.11]}$

In the presence of a negative $\eta^{4}$ contribution, the free energy may be recast into

$$
\begin{aligned}
F(T, c, \eta) & =F_{0}(T, c)+A_{2}(T, c) \eta^{2}+\left(A_{4}(T, c)-B_{4}(T, c)\right) \eta^{4}+A_{6}(T, c) \eta^{6} \\
& =F_{0}(T, c)+B(c)\left(T-T_{i}\right) \eta^{2}+C_{4}(T, c) \eta^{4}+A_{6}(T, c) \eta^{6}
\end{aligned}
$$

where $C_{4}(T, c)<0$ and $A_{6}(T, c)>0$. Fig. 2.1c) summarizes important aspects of the ordering Iransition described by eq (2.5c). By analysis similar to the one for eq (2.5b), the following relations can be attained.

$$
T_{i}^{+}=T_{i}^{*}+\frac{C_{4}^{2}}{3 B A_{6}}, \quad T_{0}=T_{i}^{-}+\frac{C_{4}^{2}}{4 B A_{6}}
$$

\footnotetext{
${ }^{t}$ In case of the $L 1_{0}$ ordered structure, $\gamma\left(2 \pi a_{3}^{*}\right)=\frac{1}{2}, \gamma\left(2 \pi a_{1}^{*}\right)=\gamma\left(2 \pi a_{2}^{*}\right)=0$ for the stoichiometric composition.
} 


\subsubsection{Mean Fleld Approximation to the IsIng Model In the Method of scW.}

Although the Landau-Lifshitz theory displays many important aspects of order-disorder transformations, it is incapable of providing quantitative predictions in a self-consistent manner. This is because the expansion coefficients introduced in the phenomenological free energy are no more than arbitrary symbols which do not carry any information on the physical nature of an alloy. In order to acquire quantitative information, a statistical approach needs to be made employing a physical model of an alloy. For this purpose, the Ising model has been utilized in numerous theoretical studies.

According to the Ising model, the configurational energy of a binary alloy on a rigid lattice is approximated as

$$
H=\frac{1}{2} \sum_{r, r} V(r, r) c(r) c\left(r^{\prime}\right)
$$

Where $c(r)$ is a stochastic variable describing the state of occupancy of the lattice site $r: c(r)=$ 1. Hite $r$ is taken by a solute atom and $c(r)=0$ otherwise. $V\left(r, r^{\prime}\right)$ is the effective pairwise interaction potential between two solute atoms at the lattice sites $r$ and $r^{r}$ and is assumed to be independent of composition and of temperature. It is further given as

$$
V(r, r)=V_{A}(r, m)+V_{B B}(r, r)-2 V_{A B}\left(r, r^{\prime}\right)
$$

where $V_{A A}(r, r), V_{B B}(r, r)$ and $V_{A B}(r, r)$ are interaction energies of respective atom pairs.

From eq. (2.12), the partition function for the canonical ensemble of the model binary alloy is then given by

$$
Z=\sum_{\left\{c\left(r_{1}\right), c\left(r_{2}\right) \ldots c\left(r_{1}\right)\right)} \exp \left(-\frac{1}{2 k T} \sum_{r, r} V(r, r) c(r) c\left(r^{\prime}\right)\right)
$$

where such combinations of $\left(c\left(r_{1}\right), c\left(r_{2}\right), \ldots c\left(r_{N}\right)\right\}$ that do not conserve the given number of solute atoms are excluded in the summation. Eq. (2.13) can be recast into: 


$$
Z=\sum_{\left\{c\left(r_{i}\right)\right)_{H}} \Omega\left(H\left(c\left(r_{i}\right)\right]\right) \exp \left(-\frac{H\left(\left[c\left(r_{i}\right)\right\}\right)}{k T}\right)
$$

where $\left\{c\left(r_{i}\right)\right\}_{H}$ denotes a set of configurational states which have the same energy $(H)$ and $\Omega$ represents the number of configurational states in the set $\left\{c\left(r_{i}\right)\right\}_{H}$. Assuming the system is large so that the canonical distribution is sharp enough around the most probable energy state the canonical partition function $Z$ can be readily approximated as (see, for example. [50] and [53])

$$
\begin{aligned}
& Z \equiv\left[\Omega\left(H\left(c\left(\mathrm{r}_{i}\right)\right\}\right) \exp \left(-\frac{H\left[\left(c\left(\mathrm{r}_{i}\right)\right]\right.}{k T}\right)\right]_{\mathrm{Mex}} \\
& =\left[\exp \left(-\frac{1}{k T}\left\langle H\left(c\left(\mathrm{r}_{\mathrm{i}}\right)\right)-k T \ln \Omega\left(c\left(\mathrm{r}_{\mathrm{i}}\right)\right)\right]\right)\right]_{\operatorname{Max}} \\
& =\left[\exp \left(-\frac{1}{k T}\left\langle H\left(c\left(r_{i}\right)\right]-T S\left(c\left(r_{i}\right)\right)\right)\right]\right]_{\text {Mex }}=\exp \left(-\frac{F_{\min }\left(\left(c\left(r_{i}\right)\right]\right)}{k T}\right)
\end{aligned}
$$

From the basic assertion of statistical mechanics,

$$
F_{\text {equil }}=-k T \ln Z
$$

where $F_{\text {equil }}$ is the Helmholtz free energy in the canonical equilibrium. Substituting eq. (2.15) into eq. (2.16) yields,

$$
F_{\text {equil }} \equiv F_{\min }\left(\left\{c\left(\mathrm{r}_{\mathrm{i}}\right) H\right\}\right)
$$

It should be noted that eq. (2.17) has been attained by the truncation of the energy states with less statistical weight in the ensemble. Even with this truncation, it is a formidable task to quantify the most probable state. In fact, the well-known various approximation methods 126. 45. 54-58] are intended to derive the free energy expressions which may best approximate the most probable state through relevant minimization procedures.

In order to attain the mean field free energy expression, further derivations could be made in the following way.

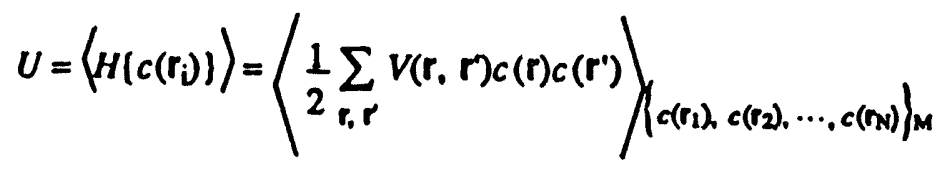

where $U$ is the internal energy and $<\ldots>$ denotes the averaging over a representative ensemble $\left\{c\left(r_{1}\right), c\left(r_{2}\right), \ldots c\left(r_{N}\right)\right\}_{M}$ where $M$ represents the most probable state which has 
been sampled during the derivation of eq. (2.15). The stochastic variable $c(r)$ may be rewritten as

$$
c(r)=p(r)+\Delta c(r)
$$

where $p(r)=\left\langle c(r)>\right.$ and $\sum_{r} \Delta c(r)=0$, by definition. Substituting eq. (2.19) into eq. (2.18) yields

$$
\begin{aligned}
U & =\left\langle\frac{1}{2} \sum_{r, r^{\prime}} V\left(r, r^{\prime}\right) c(r) c\left(r^{\prime}\right)\right\rangle=\frac{1}{2} \sum_{r, r^{\prime}} V\left(r, r^{\prime}\right)\left\langle c(r) c\left(r^{\prime}\right)\right\rangle \\
& =\frac{1}{2} \sum_{r, r} V\left(r, r^{\prime}\right)\left(p(r) p\left(r^{\prime}\right)+\left\langle\Delta c(r) \Delta c\left(r^{\prime}\right)\right\rangle\right)
\end{aligned}
$$

The quantity $\left\langle\Delta c(r) \Delta c\left(r^{\prime}\right)\right\rangle$ is called the pair correlation function and is defined as $[28,50]$

$$
\left\langle\Delta c(\mathbf{r}) \Delta c\left(\mathbf{r}^{\prime}\right)\right\rangle=\left\langle c(\mathbf{r}) c\left(\mathbf{r}^{\prime}\right)\right\rangle-p(\mathbf{r}) p\left(\mathbf{r}^{\prime}\right)
$$

In the mean field approximation, this correlation term is neglected. Accordingly,

$$
U=\frac{1}{2} \sum_{r, r} V\left(r, r^{\prime}\right) p(r) p\left(r^{\prime}\right)^{\prime}=\frac{1}{2} \sum_{r} \phi(r) p(r)
$$

where $\phi(r)=\sum V\left(r, r^{\prime}\right) p\left(r^{\prime}\right)$ is the mean potential to which an average atom at the lattice site $r$ is subjected. The average crystal thus constructed is such that each lattice site is opcupied by an average $A B$ atom each of which is subject to the mean potential $\phi(r)$ generated by all the neighboring atoms. By regarding each lattice site as an individual microscopic system, one can then attain the total configurational entropy by summing up the entropic contribution due to each microscopic system over the whole average crystal.

$$
S=-k \sum_{r}[p(r) h p(r)+(1-p(r)) h(1-p(r))]
$$

From eq.'s (2.21) and (2.22), the mean field free energy is then given by

$$
F=\frac{1}{2} \sum_{r, r} V\left(r, r^{\prime}\right) p(r) p\left(r^{\prime}\right)+k T \sum_{p}[p(r) h p(r)+(1-p(r)) h(1-p(r))]
$$

In the case where all the lattice sites of a binary alloy are described by a single Bravais lattice and the atomic distribution in the ordered phase can be represented by a single group of wave vectors belonging to the same star $\left\{k^{\circ}\right\}$, the method of SCW gives $[28,59]$ 


$$
p(r)=c+\frac{\eta}{2} \sum_{n}\left[\gamma\left(k_{n}^{0}\right) e^{2 k_{n}^{0} r}+\gamma^{*}\left(k_{n}^{0}\right) e^{-i k_{n}^{0}}\right]
$$

which is already shown in eq. (2.1) - (2.3). From this, one can attain a set of $p(r)$ 's in terms of $c$ and $\eta$ which describe the solute distribution for a given ordered structure. By substituting eq. (2.22) into eq. (2.23), a mean field free energy expression is attained which can be used to analyze various thermodynamic aspects of an order-disorder transformation in a binary alloy system.

A few shortcomings of the resulting mean field Helmholz free energy should be noted. Firstly, correlation effects are not taken into account in the derivation of the free energy. As a major outcome, the entrop's contribution to the free energy tends to be overestimated. This may not be a problem in the limiting cases of high and low temperatures but could be a source of serious errors in the vicinity of an order-disorder transition temperature where correlation effects may play an increasingly important role. ${ }^{[60,61]}$ Secondly, the free energy is due to a rigid lattice model in which each configurational state is distinguished from one another only in terms of atomic configuration on a common non-deformable lattice. Accordingly, the free energy may be used neither to study order-disorder transformations (at constant composition) which involve significant lattice distortions nor to calculate phase diagrams involving phases with significantly different lattice characteristics. The problem of rigidity can be treated explicitly as an elasticity problem. In this approach, a configurational state on the rigid Ising lattice is envisioned as being maintained strain-free by non-physical forces that prevent the relaxation of the lattice. We will give detailed consideration on this in chapter 3.

\subsection{Continum Elasticity Theory of a Plate-like Precipitate.}

A coherent product phase with a plate-like shape is frequently found to form in various solid state phase transformations of alloys. The appearance of the plate-like shape can not be explained in terms of the conventional criterion, that is, surface energy minimization. This 
is because the shape is the one that maximizes the surface area for a fixed volume. As has been illustrated in numerous experimental work, the plate-like shape may arise when the transformation to a product phase requires strong anisotropic distortion of the crystal lattice of a parent. phase. Accordingly, elasticity is a critical factor in the morphology of coherent mixture containing plate-like product phase. In this section, we present some of the important concepts of continum elasticity theories of a plate-like precipitate. The theories developed by Khachaturyan $[35,62,63]$ are selected to study which have proven to be successful in related applications $[34,63-66]$.

\subsubsection{Straln Energy of a Coherent Plate-llke Preclpitate}

Accurding to Khachaturyan [24,35], the strain energy of a coherent precipitate embedded in an anisotropic infinite matrix is given by eq. (2.25), providing that the precipitate phase has the elastic moduli identical to that of the matrix phase (homogeneous moduli approximation).

$$
E=\frac{1}{2} \iint_{-\infty}^{\infty} \frac{d^{3} k}{(2 \pi)^{3}} B(n)|\theta(k)|^{2}
$$

where $\theta(k)$ is specified by eq. (2.26)and $B(n)$ by eq. (2.27).

$$
\theta(k)=\iiint_{-\infty}^{\infty} \theta(r) e^{w} d^{3} r
$$

where $\theta(r)$ is a function describing the shape of a precipitate and it has the value of unity inside the precipitate and zero otherwise.

$$
B(n)=\lambda_{i j k l} \varepsilon_{i j}^{\circ} \varepsilon_{k l}^{\circ}-n_{i} \sigma_{i j}^{\circ} \Omega_{j l} \sigma_{m}^{0} n_{m}
$$

where $n=k / k, \lambda_{i j k l}$ is the elastic modulus tensor and $\varepsilon_{i j}^{0}$ is the strain tensor describing the geometrical difference between the crystal lattices of the matrix and the precipitate phase in their respective stress free state and $\sigma_{i j}^{\circ}$ and $\Omega_{i j}$ are tensors given by

$$
\sigma_{i j}^{0}=\lambda_{i j k l} \varepsilon_{k l}^{0}
$$




$$
\Omega_{i j}^{-1}=\lambda_{i k \xi} n_{k} n_{l}
$$

If multiplied by $1 / 2$, the first term in $B(n)$ represents the strain energy arising from the stress free transformation into a unit volume of the precipitate and the negative second term represents the strain energy associated with the relaxation of the stress free transformed volume of the precipitate through coherency with the matrix. Accordingly, $B(n)$ is positive semi-definite since otherwise, a non-physical matrix state is encountered where the matrix has a negative resistance to the relaxation of the precipitate.

For a plate-like precipitate of infinitesimal thickness, eq. (2.25) is reduced to a simple form of eq. (2.30) since $B(n)$ has a non-vanishing value only at the habit plane normal $n$.

where $V \equiv \int^{\infty} \int_{-\infty} \frac{d^{3} k}{(2 \pi)^{3}}|\theta(k)|^{2}$.

$$
E=\frac{1}{2} B(n) V
$$

When a plate-like precipitate is elastically stable by the possession of the optimum habit plane, eq. (2.30) has its minimum value: $E_{\min }=\frac{1}{2} B_{\min }\left(n_{0}\right) V$. Since this is the absolute minimum of eq. (2.25), a conclusion may be reached that the optimum shape to minimize the strain energy is ideally to be a plate-like shape for any coherent precipitate. The actual shape of a precipitate, however, is not determined solely by the condition of the elastic energy minimum but through a compromise between interphase energy and elastic energy. The relation describing the relative contribution of each term in determining the actual shape of a precipitate was derived by Khachaturyan $[35,66]$ and independently by Roitburd $[67,68]$. For example, when the stress free transformation strain is a pure dilatation, it is given by

$$
\sum=\frac{\gamma_{3}}{f\left(\lambda_{i j k \delta} \varepsilon_{0}^{2} v_{3}^{1}\right.}
$$

where $E, \Gamma, \gamma_{s}, \varepsilon_{0}, V, f\left(\lambda_{i j k t}\right)$ are strain energy, interphase energy, specific interphase energy, stress free dilatational strain, precipitate volume and a function of elastic constants $\left(\lambda_{i j k l}\right)$ with an order of one. If $\Gamma / E>1$ due to large $\gamma_{s}$, small misfit strain and small precipitate volume, a 
precipitate may form with an equiaxed shape. If $\Gamma / E \ll 1$ due to a large precipitate volume, misfit strain and small $\gamma_{s}$, the plate-like shape is preferred by a precipitate.

\subsubsection{Hablt Plane of a Plate-llke Preclpltate}

Eq. (2.30) together with eq. (2.27) show that elastic strain energy of a thin coherent platelike precipitate depends uniquely on the habit plane normal $\mathbf{n}$ for given stress free transformation strain and elastic constants. Accordingly, the habit plane which is preferred by an elastically stable precipitate can be determined by minimizing the strain energy with respect to the habit plane normal. This problem was discussed for the case of elastic isotropy [68] and for the case of elastic anisotropy $[35,63,68,69]$. Wen ef al. derived the exact solution of the problem for the case of a tetragonal plate-like precipitate coherently embedded in an anisotropic cubic matrix. [63] In the case considered, the stress free transformation strain describing the geometrical difference between the crystal lattice of the matrix and that of the precipitate is given by

$$
\tilde{\varepsilon}=\left(\begin{array}{ccc}
\varepsilon_{11}^{\circ} & 0 & 0 \\
0 & \varepsilon_{11}^{\circ} & 0 \\
0 & 0 & \varepsilon_{33}^{0}
\end{array}\right)
$$

For cubic anisotropy of the usual type, namely, $C_{11}-C_{12}-2 C_{44}<0$, the optimum habit plane resulting from the minimization of eq. (2.30) was determined to be

$$
n_{0}=(\sin \theta, Q, \cos \theta)
$$

where

$$
\cos ^{2} \theta=\left[\begin{array}{cc}
0 & \text { when }-\infty<t<-\frac{C_{11}+C_{12}}{C_{12}} \text { and } 1 \leq t \leq \infty \\
1+\frac{C_{11}+2 C_{12}}{C_{11}+C_{12}} \frac{1}{1-t} & -\frac{C_{11}}{C_{12}}-1<t<0 \\
1 & 0 \leq t<1
\end{array}\right.
$$

where $t=\frac{\varepsilon_{11}^{\circ}}{\varepsilon_{33}^{\circ}}$ and the conventions for elastic constants are $\lambda_{i j k l}=C_{11}$ for $i=j=k=l, \lambda_{i j k l}=C_{12}$ for $(i=j) \neq(k=l)$ or $(i=k) \neq(j=l)$. The components of the habit plane normal vector $n_{0}$ refer to 
the Cartesian coordinate system with axes parallel to [100], [010] and [001] directions of the crystal lattice of a stress free precipitate phase and $\theta$ is the angle measured from the [001] axis.

\subsubsection{Stablity of a Habit Plane}

In determining the optimum habit plane of a plate-like precipitate, a necessary condition is applied that the first variation of the strain energy of eq. (2.30) with respect to habit plane normal should vanish. Habit planes resulting from this condition, however, may correspond to strain energy extrema of various kinds, that is, maximum, minimum and saddle point. Accordingly, one may need a secondary analysis to identify the nature of a habit plane in terms of its relative stability. In what follows, we expand what was done in the work of Wen et al. $\{24.63\}$ by reformulation of the problem and derive the general equation which may be utilized to determine the stability of a habit plane.

Let's consider an infinitesimally thin plate-like precipitate with a habit plane for which the

strain energy of eq. (2.30) has an extremum, that is, $E\left(n_{0}\right)=\frac{1}{2} B\left(n_{0}\right) V$. Suppose that the precipitate is made to have a new habit plane with the normal vector $\boldsymbol{n}$ which is different from $n_{0}$ by an infinitesimal deviation. The unit vector $n$ normal to the new habit plane may be represented by

$$
n=n_{0}+\delta n-\frac{1}{2} \mid \delta n^{2} n_{0}
$$

where $\delta n$ is the projection of $n-n_{0}$ onto the old habit plane with $n_{0}$. The change in the strain energy associated with the variation in the habit plane normal may then be approximated as

$$
\Delta E(n)=E(n)-E\left(n_{0}\right)=\delta^{2} E(n)+\ldots
$$

where $\delta E(n)=0$ by definition and the second variation $\delta^{2} E(n)$ is given by

$$
\delta^{2} E(\mathbf{n})=\frac{V}{2} \beta_{i j} \delta n_{i} \delta n_{j} \quad(i, j=1,2)
$$

where 


$$
\beta_{i j}=\frac{1}{2}\left(\frac{\partial^{2} B(n)}{\partial n_{i} \partial n_{j}}\right)_{n=n_{0}}
$$

Due to the constraint imposed on the unit vector $n$ i.e. $|n|^{2}=1$, the variation is taken with respect to two independent components lying on the old habit plane. According to the eq. (2.37), the habit plane with the normal $n_{0}$ is elastically stable, providing that the eigenvalues of the matrix $\beta_{i j}$ are positive definite. In the homogeneous moduli approximation, our derivation of the matrix $\beta_{i j}$ leads to (see Appendix).

$$
\beta_{i j}\left(n_{0}\right)=\lambda_{i j k k} S_{k} S_{l}-\left\{\sigma_{i k}^{*} \Omega_{k l} \sigma_{j l}^{*}+\sigma_{i k}^{*} \Omega_{k}\left(\hat{\sigma}-\widehat{\sigma}^{0}\right)_{j}+\left(\hat{\sigma}-\hat{\sigma}^{0}\right)_{i k} \Omega_{k l} \sigma_{j l}^{*}+\left(\hat{\sigma}-\hat{\sigma}^{0}\right)_{i k} \Omega_{k}\left(\hat{\sigma}-\widehat{\sigma}^{0}\right)_{i j}\right\}
$$

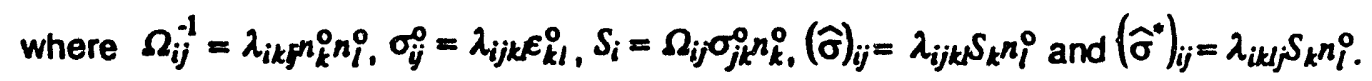

It follows from the eq. (2.39) that $\beta_{i j}$ is a function of the habit plane normal, stress free transformation strain and the elastic constants of the precipitate phase. We now apply eq. (2.39) to the simplest case, that is, $n_{0}=[001]$. When the stress free transformation strain is given by eq. (2.33), the matrix $\beta_{i j}$ has the following self-diagonalized form for $n_{0}=[001]$.

$$
\hat{\beta}=\left(\begin{array}{ll}
\beta & 0 \\
0 & \beta
\end{array}\right)
$$

where

$$
\beta=-\frac{2}{C_{44}}\left\{\left(\sigma_{11}^{\circ}\right)^{2}+\left(\sigma_{33}^{\circ}\right)^{2} \frac{C_{12}\left(C_{12}+2 C_{44}\right)}{C_{11}^{2}}-2 \sigma_{11}^{\circ} \sigma_{33}^{\circ} \frac{\left(C_{12}+C_{44}\right)}{C_{11}}\right\}
$$

Using eq. (2.28), eq. (2.40) may be recast into the form:

$$
\beta=\frac{-2\left(C_{11}-C_{12}\right)\left(C_{11}+2 C_{12}\right)^{2}\left(\varepsilon_{11}^{0}\right)^{2}}{C_{11}^{2}}\left\{\xi-\frac{2 C_{11}}{C_{11}+2 C_{12}}\left(\frac{\varepsilon_{33}^{0}}{\varepsilon_{11}^{0}}-1\right)\right\}
$$

where $\xi$ is the anisotropy constant defined by $\xi=\frac{C_{11}-C_{12}-2 C_{44}}{C_{44}}$.

Since $C_{11}>C_{12}$ in all cases, the stability condition $\beta>0$ may only be satisfied if the \{\} bracketed term is negative, that is.

$$
\xi<\frac{2 C_{11}}{C_{11}+2 C_{12}}\left(\frac{\xi_{33}^{0}}{\varepsilon_{11}^{0}}-1\right) \text { or } \frac{\varepsilon_{33}^{0}}{\varepsilon_{11}^{0}}>1+\frac{\xi}{2}\left(1+\frac{2 C_{12}}{C_{11}}\right)
$$

When the stress free transformation strain is isotropic $\left(\varepsilon_{11}^{0}=\varepsilon_{33}^{0}\right)$, this equation reproduces the well-known result i.e. $\xi<0$. 


\subsubsection{Crystal Geometry of a Constralned Plate-like Preclpitate}

The crystal lattice of a precipitate constrained by the matrix may have a geometry different from that of the precipitate lattice in the stress free state. In case of a coherent plate-like precipitate, accommodation of lattice mismatch is achieved over a broad area of a single crystallographic orientation. Accordingly, the geometry of the distorted crystal lattice of a precipitate strongly depends on the orientation of the habit plane. A detailed study of this aspect was carried out recently by Khachaturyan and Laughlin [34] in an attempt to explain the nature of various metastable phases which are encountered in the decomposition of $\mathrm{Cu}$ rich Cu-Be alloys.

According to the theories of a plate-like precipitate $[24,35,67,68]$, the elastic strain associated with the strain energy of eq. (2.30) has the following characteristics. Firstly, it is localized homogeneously inside the precipitate phase. Secondly, together with the stress free transformation strain, it provides the compatibility of the lattices across the interphase plane. This means that the total strain consisting of the elastic strain and the stress free transformation strain is invariant plane strain. From the latter, the total homogeneous strain is given by eq. (2.43) and eq. (2.44) for the habit plane normal $n_{0}$.

$$
\begin{gathered}
\varepsilon_{i j}^{l}=\varepsilon_{i j}^{o}+\varepsilon_{i j} \\
\varepsilon_{i j}^{l}=\frac{1}{2}\left(S_{i n j}^{\circ}+S_{j} n_{i}^{\circ}\right)
\end{gathered}
$$

where $\varepsilon_{i j,}^{l} \varepsilon_{i j}^{o}$ and $\varepsilon_{i j}$ are the tensors of total homogeneous strain, stress free transformation strain and elastic strain, respectively and $S_{i}$ is the $i$ th component of the shear vector $\mathbf{S}$ specified by

$$
\mathbf{S}=\widehat{\Omega}\left(\mathbf{n}_{0}\right) \widehat{\sigma} n_{0}
$$

where $\widehat{\Omega}$ and $\hat{\sigma}^{\circ}$ are defined as above (eq.'s (2.28) and (2.29)). Since the total deformation includes the rigid body rotation to maintain the continuity of the crystal lattice across the interphase,

$$
u_{i j}^{l}=S_{i} n_{j}^{0}=\frac{1}{2}\left(S_{i} n_{j}^{0}+S_{j} n_{i}^{0}\right)+\frac{1}{2}\left(S_{i} n_{j}^{0}-S_{j} n_{i}^{0}\right)
$$




$$
=\varepsilon_{i j}^{l}+\omega_{i j}
$$

where the symmetric $\left(\varepsilon_{i j}^{i}\right)$ and the antisymmetric $\left(\omega_{i j}\right)$ part describe the total homogeneous strain and the rigid body rotation, respectively. The axis and the angle of the rotation of the precipitate crystal are then determined from the direction and the magnitude of the axial vector $\varphi$ with the components specified by

$$
\varphi_{i}=\frac{1}{2} \varepsilon_{i j k} \omega_{j k}=\frac{1}{4} \varepsilon_{i j k}\left(S_{j} n_{k}^{\circ}-S_{k} n_{j}^{\circ}\right)
$$

where $\varepsilon_{i j k}$ is the Levi-Civita symbol. With the total deformation $\left(u_{i j}^{t}\right)$ known, the geometry of the crystal lattice of a constrained precipitate is completely determined using the relation:

$$
R^{P}=\hat{A} \cdot R^{m}=(\hat{I}+\underline{W}) \cdot R^{m}=\left(\hat{I}+S n_{0}\right) \cdot R^{m}
$$

where $\widehat{A}$ is the transformation matrix and $\mathbf{R}^{\mathrm{p}}, \mathbf{R}^{\mathrm{m}}$ are lattice vectors of the precipitate and the matrix lattice referred to the same reference coordinate system. The lattice rearrangement represented by the matrix $\hat{\mathbf{A}}$ gives rise to the corresponding rearrangement in the reciprocal lattice, which is described by

$$
G^{p}=\left(\hat{A}^{-1}\right)^{+} \cdot G^{m}=\left(\hat{i} \cdot \frac{n_{0} S}{1+n_{0} \cdot S}\right) \cdot G^{m}
$$

where $\left(\widehat{A}^{-1}\right)^{+}$is the transpose of the inverse of $\widehat{A}$ and $G^{P}, G^{m}$ are reciprocal lattice vectors of the precipitate and the matrix phase denoted in the same reference coordinate system. With $G^{m}$ subtracted from each side, eq. (2.49) specifies the magnitude and the direction of spot splitting in reciprocal space due to the transformation given by eq. (2.48).

\subsubsection{Shape of a Plate-llke Preclpltate}

A plate-like precipitate may not have an arbitrary shape on the habit plane. Existing experimental documentation on various shapes such as discs, polygons, and laths suggests that there exist a systematics in attainment of a particular shape out of a number of possibilities. A theory which may serve to clarify such a systematics was proposed by Khachaturyan and Hairapetyan [62].

For a plate-like precipitate of a finite thickness, eq (2.25) may be rewritten effectively into: 


$$
\begin{aligned}
& E=\frac{1}{2} \iiint_{-\infty}^{\infty} \frac{d^{3} k}{(2 \pi)^{3}}\left[B\left(n_{0}\right)+\Delta B(n)\right]|\theta(k)|^{2} \\
& =\frac{1}{2} B\left(n_{0}\right) V+\frac{1}{2} \iiint_{-\infty}^{\infty} \frac{d^{3} k}{(2 \pi)^{3}} \Delta B(n)|\theta(k)|^{2}
\end{aligned}
$$

where $\Delta B(n)$ is taken as $\Delta B(n)=B(n)-B\left(n_{0}\right)$ and $B\left(n_{0}\right)=B_{\min }(n)$ by definition. As discussed already, the first term, in effect, represents the strain energy of an infinitely large plate-like precipitate of an infinitesimal thickness. The second term is the strain energy correction due to the finite thickness of a plate and depends on the shape of a plate. According to the theory [62], the equilibrium shape of a thin plate-like precipitate is determined by the condition that the sum of shape dependent elastic energy and interphase energy of a plate be minimum at a constant precipitate volume. For a thin plate, the interphase energy contribution from the plate edge area may be neglected since

$$
\frac{\Gamma_{\text {edge }}}{\Gamma_{\text {brod }}+\Gamma_{\text {edge }}} \approx \frac{\gamma_{\text {dge }} D L}{2 \gamma_{\text {boed }} L^{2}+\gamma_{c \text { dge }} D L} \approx \frac{D}{2 L} \ll 1, \quad \text { assuming } \gamma_{\text {boed }} \approx \gamma_{e d g e} \text { and } \frac{D}{L} \ll 1
$$

where $\Gamma, \gamma, D$ and $L$ denote interphase energy, specific interphase energy, plate thickness and the characteristic length of a plate, respectively. For a precipitate with a fixed volume and thickness, broad surface area is constant regardless of the shape of the plate. Since $\Gamma_{\text {broad }}$ is fixed in such a case, the problem then comes down to the variational problem of minimizing the shape dependent elastic energy under the condition of the constant surface area.

Evaluating the second term of eq. (2.50) for an arbitrary geometry shown in Fig. 2.2 and using eq. (2.51) result in eq. (2.52) in the limiting case of $\frac{D}{L} \ll 1$.

$$
\begin{gathered}
\Delta B(n)=\frac{1}{2}\left(\frac{\partial^{2} B(n)}{\partial n_{i} \partial n_{j}}\right)_{n=n_{0}} \delta n_{i} \delta n_{j}=\beta_{i j}\left(n_{0}\right) \delta n_{i} \delta n_{j} \\
\Delta E=\oint \rho(m) d l_{m}
\end{gathered}
$$


where

$$
\rho(m)=\left(\frac{D^{2}}{4 \pi} \ln \frac{D}{L}\right) \beta_{i j}\left(n_{0}\right) m_{i} m_{j}
$$

$m_{i}$ and $m_{j}$ are components of the unit vector $\boldsymbol{m}$ which is perpendicular to the linear element $d l_{\mathrm{m}}$ of the envelope defined by $y=y(x)$. Since $\beta_{i j}$ 's have values of the order of $\lambda \varepsilon_{0}^{2}$ the approximate value of $\rho(m)$ is then estimated to be

$$
\rho(m)=\frac{\lambda\left(\varepsilon_{0} D\right)^{2}}{4 \pi} \ln L
$$

where $\lambda$ and $\varepsilon_{0}$ are typical elastic modulus and stress free transformation strain, respectively. This shows that $\Delta E$ given by eq. (2.52) represents the energy of a dislocation bop with the Burgers vector of the order of $\varepsilon_{0} D$ whose line tension coefficient is given by $\rho(m)$.

If the Cartesian axes $x$ and $y$ are chosen to lie along the principal axes of the second rank tensor $\beta_{i j}$, eq. (2.53b) may be rewritten as

$$
\rho(m)=\frac{D^{2}}{4 \pi} \ln \frac{L}{D}\left(\beta_{x} m_{x}^{2}+\beta_{y} m_{y}^{2}\right)
$$

where $\beta_{x}$ and $\beta_{y}$ are the eigen values of the sucond rank tensor $\beta_{i j}$. Substitution of eq. (2.53c) together with the specific expressions of $m_{x}, m_{y}$ and $d l_{m}$ into eq. (2.52) leads to the equation to be solved in order to determine the equilibrium shape of a plate-like precipitate.

$$
\begin{gathered}
\Delta E=\frac{D^{2}}{4 \pi} \ln \frac{L}{D}\left\{\beta_{x}\left(\int \frac{\left(d y_{+} / d x\right)^{2}}{\sqrt{1+\left(d y_{+} / d x\right)^{2}}} d x+\int \frac{(d y \cdot / d x)^{2}}{\sqrt{1+(d y \cdot / d x)^{2}}} d x\right)+\right. \\
\left.\beta_{y}\left(\int \frac{d x}{\sqrt{1+\left(d y_{+} / d x\right)^{2}}}+\int \frac{d x}{\sqrt{1+(d y-/ d x)^{2}}}\right)\right\}
\end{gathered}
$$

Solving the Euler-Lagrange differential equations resulting from the minimization of eq. (2.54) under the constraint of the constancy of the broad surface area yields the following parametric equations describing the optimum shape of a plate on the habit plane. 



$$
\begin{aligned}
& \frac{y^{\prime}}{\left(1+y^{2}\right)^{\frac{1}{2}}}+\frac{\alpha y^{\prime}}{\left(1+y^{.2}\right)^{3}}= \pm x x \\
& \frac{1-2 \alpha}{\left(1+y^{2}\right)^{\frac{1}{2}}}+\frac{\alpha}{\left(1+y^{.2}\right)^{\frac{3}{2}}}= \pm x y
\end{aligned}
$$

where $y^{\prime}=d y / d x, \alpha=\frac{\beta_{x}-\beta_{y}}{\beta_{x}}\left(\beta_{x}>\beta_{y}>0\right)$ and $x$ is the Lagrange multiplier introduced during the minimization of eq. (2.54).

For an application of the theory, one needs a specific expression for $\beta_{i j}$ which takes account of the effect of elastic constants as well as that of the transformation crystallography on the shape of a plate-like precipitate. It has been derived by Khachaturyan and Rumynina [65] during their theoretical analysis of the shape of a martensite lath in Fe-Ni alloys.

$$
\beta_{i j}\left(n_{0}\right)=\varepsilon_{0}^{2} \Omega_{i j}^{-1}(I)-\left(\hat{\sigma}_{1} \hat{\Omega}\left(n_{0}\right) \tilde{\sigma}_{1}^{+}\right)_{i j}
$$

where $\varepsilon_{0}$ and I are the characteristic homogeneous strain and the unit vector along the shear direction in the martensite transformation. $\hat{\sigma}_{1}$ is defined by eq. (2.57) and $\hat{\sigma}_{1}$ is the Hermitian of $\hat{\sigma}_{1}$.

$$
\left(\hat{\sigma}_{1}\right)_{i j}=\varepsilon_{0} \lambda_{i k g} l_{k} n_{i}
$$

Eq. (2.57) is, however, only valid for a stress free transformation in which the invariant plane distortion is described by $u_{i j}=\varepsilon_{0} l_{i} n_{j}$ (compare with eq. (2.46)). In order to calculate the shape of a coherent plate-like precipitate, a different expression is required. In the homogeneous moduli approximation, $i t$ is given by eq. (2.39) which we derived in the section 2.2.3. 


\title{
Chapter 3
}

\section{Thermodynamic Consideration of the Tetragonal Lattice Distortion of the $L_{1}$ phase.}

\begin{abstract}
In this chapter, we examine the tetragonal lattice distortion of the $L 1_{0}$ phase in terms of its effect on single and two phase equilibria in a fcc lattice. In section 3.1, a formulation is proposed to treat the problem of rigidity of the Ising model. We present the formulation in a form which can be directly used to study incoherent two phase equilibrium. In section 3.2, the formulation is specialized to derive the free energies of the $<001\rangle^{*}$ special point ordered structures within the framework of the SCW mean field approxiamtion. We then discuss in section 3.3 fundamental properties of the resulting free energies, as applied to single and two phase equilibrium involving the $L 1_{0}$ ordered phase.
\end{abstract}

\subsection{General formulation of Incoherent Two Phase Equilibrium.}

Let us suppose a hypothetisal two phase system of a binary alloy where atoms are configured on a rigid lattice to form two separate regions; one is a disordered phase with the solute concentration $c_{d}$ and the other an ordered phase with the solute concentration $c_{0}$ and the long range order parameter $\eta$ (see Fig. 3.1). By 'rigid lattice', it is supposed that each phase is forced to remain strain free on the pure solvent lattice: a different choice of a rigid lattice e.g. the lattice of a disordered phase of composition $c_{d}$, does not affect the equilibrium properties of free energies. The configurational free energy of each phase on the rigid lattice, that is, $F_{\text {ngid }}^{\text {did }}$ for a disordered phase and $F_{\text {ngid }}^{\circ}$ for an ordered phase, is given by a free energy model due to the rigid lattice Ising model (eq. (2.12)). The resulting Helmholtz free energy may not be used to describe a phase in its stress free state particularly when the stress free 


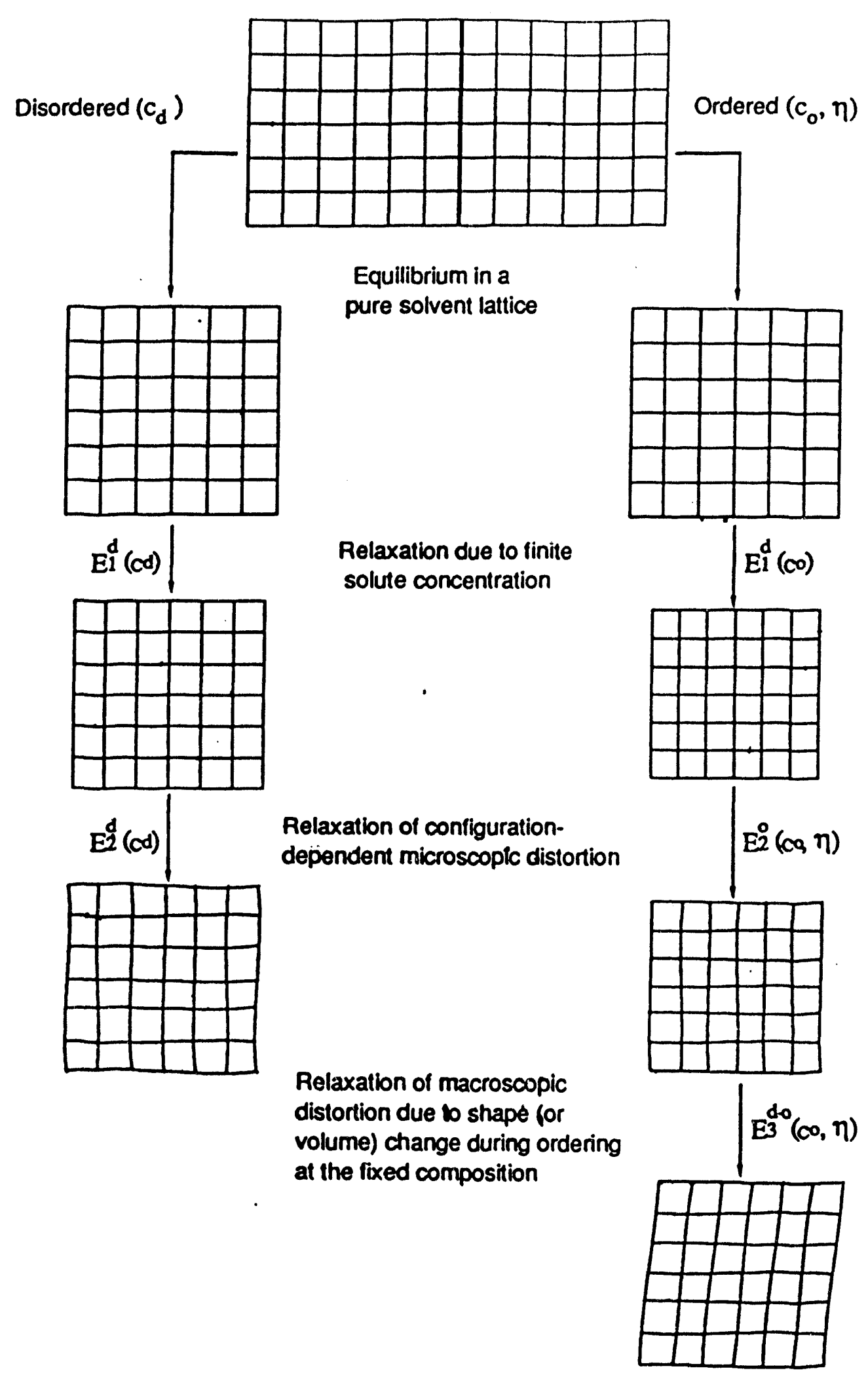

Figure 3.1: Schematic diagram illustrating renormalization of rigid lattice free energies into stress free lattice equivalents. 
crystal lattice is significantly different from that of the strain free rigid one. Given the Helmholtz free energy, the configurational free energy of a stress free phase can be attained through relaxation of non-physical forces which are responsible for the rigidity of the strain free lattice. The process depicted in Fig. 3.1 may be formulated as

$$
\begin{gathered}
F^{\mathrm{d}}\left(c_{\mathrm{d}}\right)_{\sigma^{\alpha}=0}=\left.F_{\text {rgid }}^{\mathrm{d}}\left(c_{\mathrm{d}}\right)\right|_{\varepsilon=0}+\sum_{i} E_{i}^{\mathrm{d}}\left(c_{\mathrm{d}}\right) \\
F^{\circ}\left(c_{0,}, \eta\right)_{\sigma^{\circ}=0}=F_{\text {rgid }}^{\mathrm{O}}\left(c_{0}, \eta\right)_{\varepsilon=0}+\sum_{i} E_{i}^{\mathrm{O}}\left(c_{0}, \eta\right)
\end{gathered}
$$

where $\varepsilon=0$ and $\sigma=0$ denote the strain free solvent rigid lattice and the stress free crystal lattice of the phase in concem, respectively and $E_{i}$ represents the elastic energy relaxation due to a source $i$. For the ordered phase, the origins of elastic energy relaxation may consist of:

1) homogeneous lattice distortion due to finite solute concentration.

2) localized microscopic lattice distortion associated with a spatial distribution of atoms of different sizes.

3) macroscopic lattice distortion resulting from the difference between the stress free crystal lattice of the ordered phase and that of the disordered phase of the same solute concentration.

As it turns out, lattice distortion due to the sources 2 and 3 depends on the state of order whereas distortion due to the source 1 does not.

Relaxation of the elastic energy associated with the sources 1 and 2 may be properly described by the microscopic elasticity theory developed by Khachaturyan $[24,70]$.

$$
\begin{gathered}
E_{1}=-\frac{N}{2}(M(k)\rangle c+\frac{N}{2}\left[-v_{0} C_{i j k l} \varepsilon_{i j}^{0} \varepsilon_{k l}+\langle M(k))\right] c^{2} \\
E_{2}=\frac{1}{2 N} \sum_{k}[-M(k)+\langle M(k)\rangle]|\delta c(k)\rangle^{2}
\end{gathered}
$$

where $c_{1} v_{0}, N, C_{i j k l}$ and $\varepsilon_{i j}^{0}$ are, respectively, average solute concentration, volume of a solvent atom, total number of atoms, elastic constants and the stress free transiormation strain accounting for the difference in the crystal lattice of the pure solvent and that of the pure solute. The strain $\varepsilon_{i j}^{0}$ is given by eq. (3.5) when the composition dependence of the 
lattice constants is assumed to follow the Vegard's law. $M(k), \angle M(k)>$ and $\delta c(k)$ are specified by eq.'s (3.6), (3.7) and (3.8), respectively.

$$
\begin{gathered}
\varepsilon_{i j}^{0}=\frac{d a}{d d} \delta_{i j} \\
M(k)=F_{i}(k) G_{i j}(k) F_{j}^{*}(k) \\
\langle M(k)\rangle=\frac{1}{N} \sum_{k} F_{i}(k) G_{i j}(k) F_{j}^{*}(k) \\
\delta c(k)=\sum_{\mathbf{r}}(c(r)-c) e^{-k \cdot r}
\end{gathered}
$$

where $c(\mathbf{r}), F_{i}(\mathbf{k})$ and $G_{i j}(\mathbf{k})$ are solute concentration at lattice site $\mathbf{r}$, $i$ th component of the Fourier transform of the Kanzaki force and the Born-yon Karman tensor of the solvent lattice. Each of the latter two describes the force acting upon the solvent lattice by a solute atom and the stiffness of the solvent lattice against displacements, respectively.

The elastic strain associated with the source 3 is macroscopic and depends on the degree of long range order of the ordered phase. When the average atomic configuration of the ordered phase can be represented by a single long range order parameter, the stress free transformation strain may be effectively described by eq. (3.9).

$$
\bar{\varepsilon}_{i j}=\left.\frac{1}{2} \frac{\partial^{2} \vec{\varepsilon}_{i j}}{\partial \eta^{2}}\right|_{\eta=0} \eta^{2}+\ldots
$$

In the expansion, the coefficient of the first order term is identically zero due to the strain invariance with respect to the translation of the disordered lattice. The change in elastic energy associated with the relaxation of the homogeneous strain $\bar{\varepsilon}_{i j}$ is then given by

$$
E_{3}=-\frac{N \psi}{2} C_{i j k l} \bar{\varepsilon}_{i j}-\bar{\varepsilon}_{k l}
$$

where $v$ is the average atomic volume of a stress free disordered phase with the solute concentration $c_{0}$. Due to the relaxation represented by eq. (3.3), the volume $v$ now depends on the solute concentration and this needs to be taken into account in eq. (3.10). Assuming 
that the lattice parameters of a disordered phase vary according to Vegard's law, the average atomic volume $v$ may be expressed by eq. (3.11) for a lattice with cubic symmetry.

$$
v=\frac{1}{n}\left[a_{c=0}\left(1+\frac{d a}{a d c} c\right)\right]^{3}
$$

where $n$ is the number of atoms in a unit cell of a disordered phase.

When the relaxations represented by eq. (3.3), (3.4) and (3.10) are considered, the configurational free energy of each phase renormalized with respect to its stress free crystal lattice is determined from eq.'s (3.1) and (3.2) to be:

$$
\begin{aligned}
& F_{\text {inooh }}^{d}\left(c_{d}\right)=\left.F_{\text {ngid }}^{d}\left(c_{d}\right)\right|_{\varepsilon=0}+E_{1}^{d}\left(c_{d}\right)+E_{2}^{d}\left(c_{d}\right) \\
& F_{\text {inoob }}^{\text {o }}\left(c_{0}, \eta\right\rangle=F_{\text {igid }}^{\circ}\left(c_{0}, \eta\right)_{\varepsilon=0}+E P\left(c_{0}, \eta\right)+E q\left(c_{0}, \eta\right)+E \xi\left(c_{0}, \eta\right)
\end{aligned}
$$

The free energy with a form similar to eq. (3.13; was suggested initially by Kajitani and Cook in their study on the nature of CuAul, CuPt and CuZn type ordering transitions in equiatomic alloys [11]. Since the treatment was concerned with single phase ordering transitions, their free energy expression did not include our first relaxation term. An incoherent equilibirium between an ordered and a disordered phase can now be determined by use of the free energies specified by eq. (3.12)and (3.13). When a mean field approximation is used to

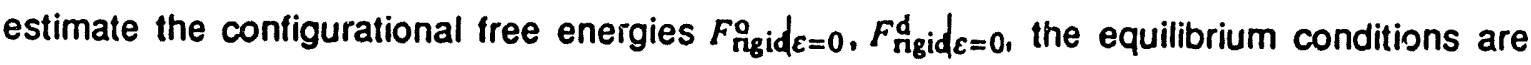
given by the followings.

At a temperature $T$.

$$
\begin{aligned}
& \frac{\partial F_{\text {mooh }}^{\circ}}{\left.\partial \eta\right|_{T . c=c_{0}} \eta=\eta}=0 \\
& \frac{\partial F_{\text {inoonh }}^{\text {on }}}{\left.\partial \eta\right|_{T, c=c_{0} \eta=\eta}}=\left.\frac{\partial F_{\text {inooh }}^{d}}{\partial \eta}\right|_{T, c=c_{d}}=\mu
\end{aligned}
$$

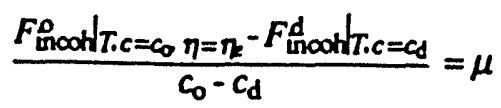


where $\eta_{e}$ and $\mu$ are equilibrium long range order parameter and chemical potential respectively. For incoherent equilibrium between two different ordered phases, substitution of

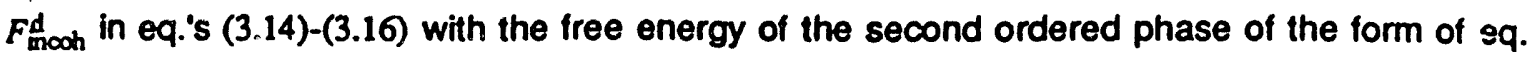
(3.13) will lead to the relevant equilibrium conditions.

\subsection{Free Energles $\left(f_{\text {Incoh }}\right)$ of $\left.<001\right\rangle^{*}$ fce Special Point Ordered Structures.}

Two distinct ordered structures are associated with the <001>* special point ordering in icc lattices, that is, $L 1_{0}$ and $L 1_{2}$. In what follows, we derive detailed expressions of the free energies which may be used to study incoherent equilibria involving the $L_{1}$ and/or $L_{2}$ ordered phases.

\subsubsection{Formulation of $\left.F_{\text {rigld }}\right|_{\varepsilon=0}$}

From the mean field approximation to the rigid lattice Ising model, the free energy describing a strain free configurational state of a AB binary alloy is given by (eq. (2.23))

$$
F_{\text {rigid }}=\frac{1}{2} \sum_{r, r} V\left(r, r^{\prime}\right) p(r) p\left(r^{\prime}\right)+k T \sum_{r}[p(r) \ln p(r)+(1-p(r)) \ln (1-p(r))]
$$

where $p(r)$ is the occupation probability of the lattice site $r$ by a solute atom and $V(r, r)$ is a pairwise interaction energy between two solute atoms at laftice sites $r$ and $r$ and is defined as $V(r, r)=V_{A A}(r, r)+V_{B B}(r, r)-2 V_{A B}(r, r)$. By use of the SCW description [24,28,48], the occupation probability $p(r)$ can be represented by eq. (3.18a) and (3.18b) for the $<001\rangle^{\circ}$ special point ordering in foc lattices.

$$
\begin{gathered}
p(r)=c+\sum_{n} Q\left(k_{n}\right) e^{a k_{n} r} \\
Q\left(k_{n}\right)=\gamma\left(k_{n}\right) \eta
\end{gathered}
$$

where $k_{n}=a_{n}^{*}(n=1,2,3)$ are the concentration wave vectors belonging to the <001>* star and $\gamma\left(k_{n}\right)$ 's are the coefficients which reflect the point group symmetry of an ordered phase 
generated by the $<001>^{*}$ star. $Q\left(k_{n}\right)$ represents the amplitude of the concentration wave of the wave vector $k_{n}$. The position vector $r$ may be expressed in terms of the fundamental lattice translation vectors $a_{1}, a_{2}$ and $a_{3}$ satisfying the relation $a_{n}^{*} \cdot a_{m}=2 \pi \delta_{n m}$.

$$
r=x a_{1}+y a_{2}+z a_{3}
$$

where $x, y$ and $z$ represent the coordinates of atomic sites in a fcc unit cell. Substituting eq. (3.19) with the site coordinates specified for each ordered structure into eq. (3.18) yields eq.'s (3.20a) and (3.20b) which describe the solute distribution in the $L 1_{0}$ and the $L 1_{2}$ ordered structure, respectively.

$$
\begin{gathered}
p(z=0)=c+c \eta, p\left(z=\frac{1}{2}\right)=c-c \eta \\
p\left(\{000\rangle_{\text {veriocs }}\right)=c+3 c \eta, p\left(\left\{\frac{11}{22} 0\right\}_{\text {froc centers }}\right)=c-c \eta
\end{gathered}
$$

where $\gamma$ was chosen to be $\gamma=c$. By this choice, the concentration dependence of $\eta$ can be effectively separated out without changing the amplitude of the concentration wave. The configurational free energies of $<001>^{*}$ ordering in rigid fcc lattices are then attained by substituting eq. (3.18) into eq. (3.17) and with the aid of eq.'s (3.20a) and (3.20b).

$$
\begin{gathered}
F_{\text {rigid }}^{L l_{1}}=\frac{N}{2}\left[c^{2} V(0)+c^{2} \eta^{2} V\left(k^{0}\right)\right]+\frac{N k I}{2}[(c+c \eta) \ln (c+c \eta)+(1-(c+c \eta)) \ln (1-(c+c \eta))+ \\
(c-c \eta) \ln (c-c \eta)+(1-(c-c \eta)) \ln (1-(c-c \eta))] \\
F_{\text {rigid }}^{L l_{2}}=\frac{N}{2}\left[c^{2} V(0)+3 c^{2} \eta^{2} V\left(k^{0}\right)\right]+\frac{N k I}{4}[(c+3 c \eta) \ln (c+3 c \eta)+(1-(c+3 c \eta)) \ln (1-(c+3 c \eta))+ \\
3(c-c \eta) \ln (c-c \eta)+3(1-(c-c \eta)) \ln (1-(c-c \eta))]
\end{gathered}
$$

where $V\left(k_{1}\right)=V\left(k_{2}\right)=V\left(k_{3}\right)=V\left(k^{0}\right)=\sum_{r} V(r) e^{-i k+}$ and $V(0)=\sum_{r} V(r)$

\subsubsection{Formulation of $E_{1}+E_{2}$}


The sum of the elastic energy relaxation $E_{1}$ (eq.(3.3)) and $E_{2}$ (eq.(3.4)) can be reduced to eq. (3.24) using the identity given by eq. (3.23).

$$
\begin{gathered}
\frac{1}{N} \sum_{k}|\delta c(k)|^{2}=N c(1-c) \\
E_{1}+E_{2}=-\frac{N}{2} v_{0} C_{i j k l} \varepsilon_{i j}^{0} \varepsilon_{k l} c^{2}-\frac{1}{2 N} \sum_{k} M(k)|\delta c(k)|^{2}
\end{gathered}
$$

Taking the ensemble average of eq. (3.24) and using the mean field approximation lead to

$$
\left.\left|E_{1}+E_{2}\right|_{E}=-\frac{N}{2} v_{0} C_{i j k d} \varepsilon_{l j}^{f} \varepsilon R_{l} c^{2}-\frac{1}{2 N} \sum_{k} M(k)\right\}\langle\delta c(k))_{E}^{2}
$$

where $<>_{E}$ denotes an average over the Gibbs ensemble. Neglecting contributions other than from $k=k^{\circ}$ and using the identity of eq. (3.26) yield eq. (3.27).

$$
\begin{gathered}
\left|\left\langle\delta c\left(k^{\circ}\right)\right\rangle_{E}\right|^{2}=(N c \eta)^{2} \\
\left|E_{1}+E_{2}\right|_{E}=-\frac{N}{2} v_{0} C_{i j k l<} \varepsilon_{i j}^{0} \varepsilon k_{l} c^{2}-\frac{N}{2} c^{2} \eta^{2} F_{i}\left(k^{\circ}\right) G_{i j}\left(k^{0}\right) F_{i}^{*}\left(k^{0}\right)
\end{gathered}
$$

where $k^{\circ}$ and $\eta$ are defined as in eq. (3.21) and (3.22). Since $F\left(k^{\circ}\right) \equiv 0$ for the special point ordering wave $k^{\circ}[71]$, eq. (3.27) reduces to a simple form given by

$$
\left|E_{1}+E_{2}\right|_{\varepsilon}=-\frac{N}{2} v_{0} C_{i j k l} \varepsilon_{i j}^{0} \varepsilon_{l} c^{2}
$$

It follows from eq. (3.28) that the elastic energy contribution $\left\langle E_{1}+E_{2}\right\rangle_{E}$ does not depend on the long range order parameter $\eta$.

\subsubsection{Formulation of $E_{3}(\eta)$}

By use of the SCW description, eq. (3.9) may be rewritten as

$$
\bar{\varepsilon}_{i j}=\left.\frac{1}{2} \frac{\partial^{2} \bar{\varepsilon}_{i j}}{\partial Q\left(k^{\circ}\right)^{2}}\right|_{\eta=0} \mid Q\left(k^{0}\right)^{2}+\ldots
$$

For the $L 1_{0}$ phase, the homogeneous strain $\bar{\varepsilon}_{i j}$ may be approximated by eq. (3.29) when the contributions from the higher order terms are neglected. 


$$
\bar{\varepsilon}_{i j} \approx \beta_{i j} c^{2} \eta^{2}
$$

where $\beta_{i j}$ is the coefficient of the second order term in the expansion and $Q\left(k^{\circ}\right)=c \eta$ defined as in eq.(3.20)'s. By use of the condition $\bar{\varepsilon}_{i, \eta=1, c=c m=0 s}=c_{s e}^{2} \beta_{i j}=\bar{\varepsilon}_{i j}^{8}$, eq. (3.29) can be rewritten as

$$
\bar{\varepsilon}_{i j}=c_{s i}^{-2} \bar{\varepsilon}_{i j} c^{2} \eta^{2}
$$

where

$$
\begin{aligned}
& \left(\bar{\varepsilon}_{i j}^{o}\right)=\left(\begin{array}{ccc}
\bar{\varepsilon}_{11}^{\rho} & 0 & 0 \\
0 & \bar{\varepsilon} \rho_{1} & 0 \\
0 & 0 & \bar{\varepsilon}_{33}^{\circ}
\end{array}\right) \\
& \bar{\varepsilon}_{11}^{o}=\frac{a_{\text {ord }}-a_{\text {dis }}}{a_{d i s}}, \bar{\varepsilon}_{33}^{\rho_{3}}=\frac{c_{\text {ord }}-a_{\text {dis }}}{a_{\text {dis }}}
\end{aligned}
$$

$a_{\mathrm{dis}}, a_{\mathrm{ord}}$ and $c_{\mathrm{ord}}$ are the lattice parameters of the disordered $f c c$ and of the perfectly ordered $L 1$ o phase of the equiatomic composition $\left(c_{s t}=0.5\right)$.' Substitution of eq. (3.30) into eq. (3.10) yields

$$
E_{3}=-\frac{N v c_{s i}^{-4}}{2} C_{i j k l} \bar{\varepsilon}_{i j}^{-\rho} \bar{\varepsilon}_{k l}^{o} c^{4} \eta^{4}
$$

where $\bar{\varepsilon}_{i j}$ and $v$ are given by eq. (3.31a,b) and eq. (3.11), respectively.

It can be easily shown that eq. (3.32) holds equally for the $L 1_{2}$ phase when $c_{s t}$ and $\bar{\varepsilon}_{i j}^{o}$ are properly replaced. Although the relative magnitude varies among different alloy systems, $\bar{\varepsilon}_{i j}^{o}$ for the $L_{2}$ ordering is, in general, negligibly small as compared with that for the $L 1_{0}$ ordering (for example, it is $-7.5 \times 10^{-4}$ for the $\mathrm{CuAu}_{3}$ phase ${ }^{(72)}$ ). For this reason, $E_{3}(\eta)=0$ will be assumed for the $\mathrm{L}_{2}$ phase.

\subsubsection{Conflgurational Free Energles (f) In Stress Free States}

The configurational free energies of the fcc disordered, $L_{1}$ and $L 1_{2}$ ordered phases in their respective 'stress free state are now given by eq. (3.33), (3.34) and (3.35) , respectively, per a lattice point. 


$$
\begin{aligned}
& f_{f c c}=\frac{1}{2} c^{2} V(0)+k T[c \ln c+(1-c) \ln (1-c)+]-\frac{v_{0}}{2} C_{i j k l} \varepsilon_{l j}^{0} \varepsilon q_{l} c^{2} \\
& f_{L_{10}}=\frac{1}{2}\left[c^{2} V(0)+c^{2} \eta^{2} V\left(k^{0}\right)\right]+\frac{k T}{2}[(c+c \eta) \ln (c+c \eta)+ \\
& (1-(c+c \eta)) \ln (1-(c+c \eta))+(c-c \eta) \ln (c-c \eta)+(1-(c-c \eta)) \ln (1-(c-c \eta))] \\
& -\frac{v_{0}}{2} C_{i j k l} \varepsilon_{i j}^{0} \varepsilon q_{l} c^{2}-\frac{v c_{j}^{-4}}{2} C_{i j k l}-\bar{\varepsilon}_{i j}^{g} \bar{\varepsilon}_{k l} c^{4} \eta^{4} \\
& f_{1_{2}}=\frac{1}{2}\left[c^{2} V(0)+3 c^{2} \eta^{2} V\left(k^{0}\right)\right]+\frac{k T}{4}[(c+3 c \eta) \ln (c+3 c \eta)+ \\
& (1-(c+3 c \eta)) \ln (1-(c+3 c \eta))+3(c-c \eta) \ln (c-c \eta)+3(1-(c-c \eta)) \ln (1-(c-c \eta))] \\
& -\frac{v_{0}}{2} C_{i j k l \ell} \varepsilon_{l j}^{0} \varepsilon_{k l} c^{2}
\end{aligned}
$$

\subsection{Results and Discussion}

As we have seen from eq. (3.33) to (3.35), by virtue of the special point symmetly, the relaxation term $\left\langle E_{1}+E_{2}\right\rangle_{E}$ is only a function of the solute concentration (quadratic in $c$ assuming elastic constants are concentration-independent) and does not depend on the long range order of the ordered state. The net effect of the term is to reduce the $V(0)$ value which describes the resistance of a perturbed solution to the development of the concentration fluctuations of a long wave length. This means that the addition of the term results in the increase in the decomposition tendency of an off-stoichiometric solution and. therefore, a wider two phase field in the equilibrium phase diagram. Since the relaxation term has little qualitative significance, we ignore it in the present study. With the term $\left\langle E_{1}+E_{2}\right\rangle_{E}$ left out, we also neglect the concentration dependence of the atomic volume $(v)$ of a disordered phase specified by eq. (3.11) for physical consistency of the model. When the concentration dependence of the atomic volume and that of elastic constants are not taken into account, eq. (3.32) can be recast into a simple form (per a lattice point) given by 


$$
e_{3}^{1-10}=-16 e e^{4} \eta^{4}
$$

where $e$ is a constant specified by eq. (3.37) when the elastic stiffness tensor of the $L_{0}$ phase is assumed to have the cubic anisotropy $\left(C_{11}=C_{1111}=C_{2222}=C_{3333}\right.$ and $C_{12}=C_{1122}=$ $C_{1133}=C_{2233}$.

$$
e=v C_{11}\left\{\left(1+\frac{C_{12}}{C_{11}}\right)\left(\bar{\varepsilon}_{11}^{0}\right)^{2}+2 \frac{C_{12}}{C_{11}} \bar{\varepsilon}_{11}^{0} \bar{\varepsilon}_{33}^{0}+\frac{1}{2}\left(\bar{\varepsilon}_{33}^{0}\right)^{2}\right\}
$$

By virtue of the simplification of eq. (3.36), we can rewrite eq.'s (3.33), (3.34) and (3.35) into a reduced form of eq. (3.38) by use of the reduced parameters defined by eq. (3.39a) - (3.39c).

$$
\begin{aligned}
& f_{i}^{*}=\frac{f_{i}}{\left|V\left(\mathbf{k}^{\circ}\right)\right|} \\
& \omega=\frac{V(0)}{\left|V\left(\mathbf{k}^{\circ}\right)\right|} \\
& \tau=\frac{k \dot{r}}{\left|V\left(\mathbf{k}^{\circ}\right)\right|} \\
& \alpha=\frac{e}{\left|V\left(\mathrm{k}^{\circ}\right)\right|}
\end{aligned}
$$

where $f_{i}$ denotes a free energy of a phase $i$. given by eq.(3.33) to (3.35) with relaxation term $\left\langle E_{1}+E_{2}\right\rangle_{E}$ being omitted and $e$ is given by eq. (3.37). The results of calculations are now in order.

\subsubsection{Long Range Order Parameter of the Stress Free L1o Phase}

The temperature dependence of the equilibrium long range order parameter of the $L_{1}$ phase can be determined numerically using eq.'s (3.14) and (3.34). The results are plotted in Fig.3.2 for the equiatomic alloy composition $(c=0.5)$ for three different $\alpha\left(=\frac{e}{\left|V\left(k^{\circ}\right)\right|}\right)$ values. Curve $A$ represents the case where the lattice of the $L 1_{0}$ ordered phase is not relaxed from the strain free condition. The monotonic decrease of the long range order parameter with increasing temperature shows that the ordering transition is of the second order. When $\alpha$ 


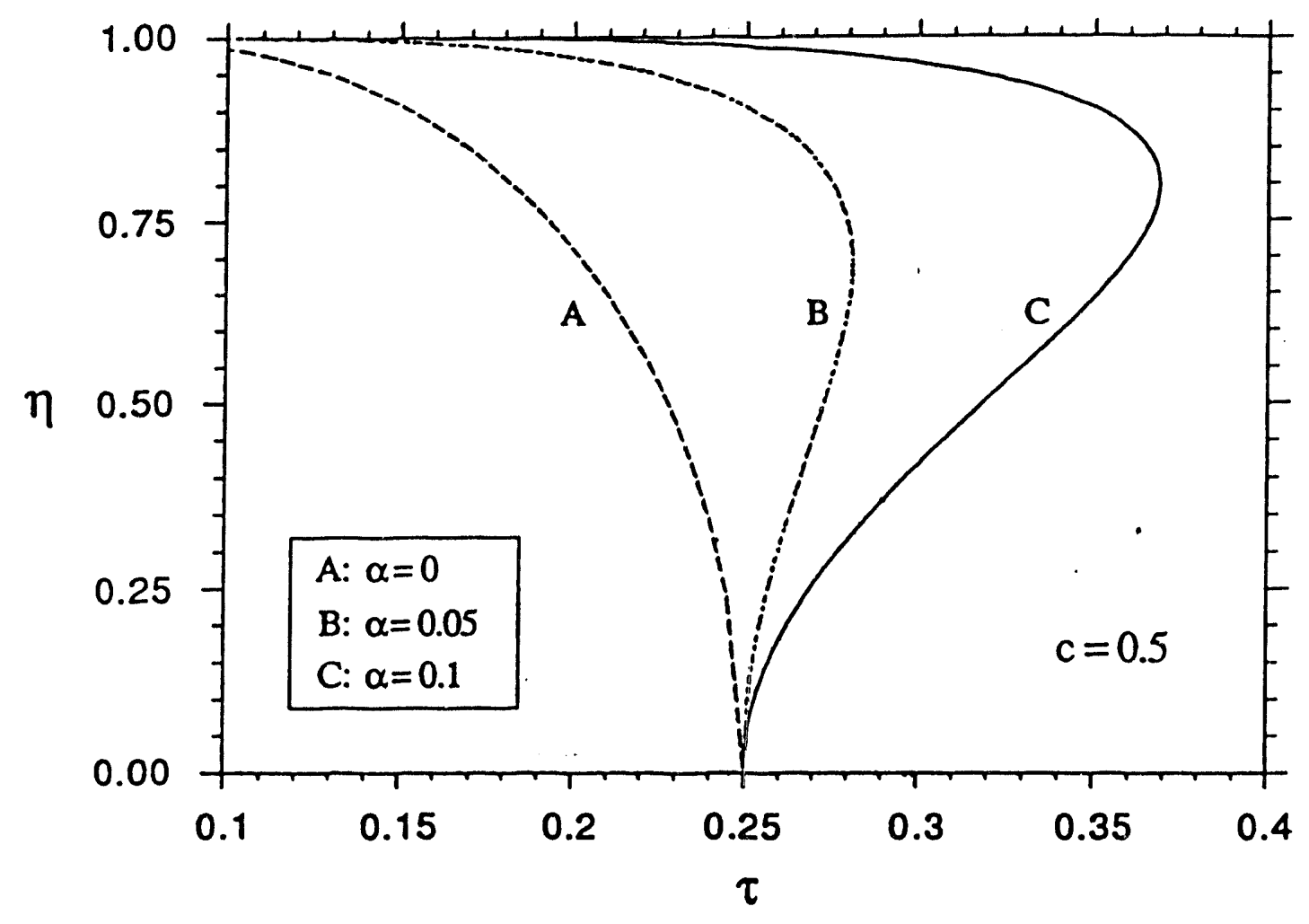

Figure 3.2: Temperature $(\tau)$ dependence of the long range onder parameter $(\eta)$ for various $\alpha$ values. When the tetragonal distortion is suppressed $(\alpha=0)$, the $L 1_{0}$ ordering transition is predicted to be thermodynamically of second order (A). As $\alpha$ increases, the ordering transition changes its character to weakly first order (B) and to strongly first order (C). This indicates that the problem of a rigid lattice model becomes increasingly serious as $\alpha$ value of an $\mathrm{L}_{0}$ ordering alloy becomes larger. 
exceeds a certain threshold value which we will discuss later, the long range order parameter curves ( $B$ and $C$ ) bifurcate into two branches above a certain temperature which defines the critical temperature of the second order transition for the $\alpha=0$ case (curve A). This bifurcation indicates that the transition now has a first order nature. Three characteristic temperatures can then be defined $[48,73]$ and they are depicted for the $\alpha=0.1$ case in Fig. 3.3a). The nature of each characteristic temperature can be displayed expressly by means of the associated $\Delta f^{*}$ vs. $\eta$ plot where $\Delta f^{*}$ is defined to be $\Delta f^{*}=f_{L_{1}}^{*}-f_{f c c}$. This is shown in Fig. 3.3b). For a given composition $c_{,} \tau_{i}$ and $\tau_{i}^{+}$represent the limit of metastablity of the disordered $f c c$ phase and of the ordered $L 1_{0}$ phase, respectively and $\tau_{0}$ is the temperature at which the free energies of the two phases become identical. Accordingly, when $\tau_{0}<\tau<\tau_{t}^{+}$. the ordered $L_{1}$ phase is metastable with respect to the disordered fcc phase of the same composition and when $\tau_{i}<\tau<\tau_{0}$, the reverse is the case.

As has been indicated previously $[10,11]$, the change in the thermodynamic order of the transition from the first to the second is directly associated with the negative anharmonic fourth order $\left(\eta^{4}\right)$ term in the free energy of eq. (3.34). By analyzing the expansion of the change of free energy $\Delta^{*}$ in terms of the long range order parameter, more insight can be gained on the features displayed in Fig. 3.2 and 3.3.

$$
\Delta f^{*}=A_{2} \eta^{2}+A_{4} \eta^{4}+A_{6} \eta^{6}+\ldots
$$

where the coefficients $A_{2}, A_{4}$ and $A_{6}$ are specified by eq.(3.41a)-(3.41c).

$$
\begin{aligned}
& A_{2}=\frac{\pi c^{2}}{2}\left[\frac{1}{c(1-c)}-\frac{1}{\tau}\right] \\
& A_{4}=\frac{2 \pi c^{4}}{4 !}\left[\frac{1}{c^{3}}+\frac{1}{(1-c)^{3}}\right]-16 \alpha c^{4} \\
& A_{6}=\frac{4 ! \pi c^{6}}{6 !}\left[\frac{1}{c^{5}}+\frac{1}{(1-c)^{5}}\right]
\end{aligned}
$$

3.3.1.1. $\tau_{i}^{*}, \tau_{i}^{+}$and $\tau_{0}$ 


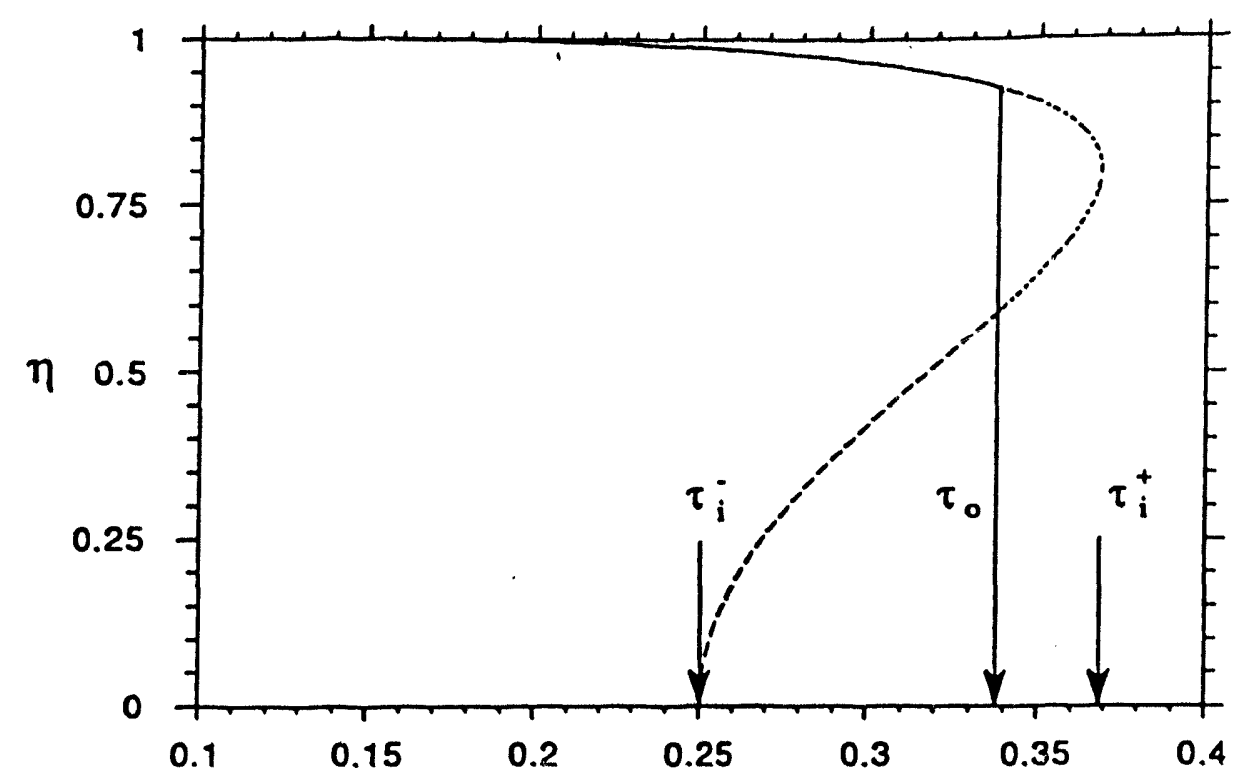

a)

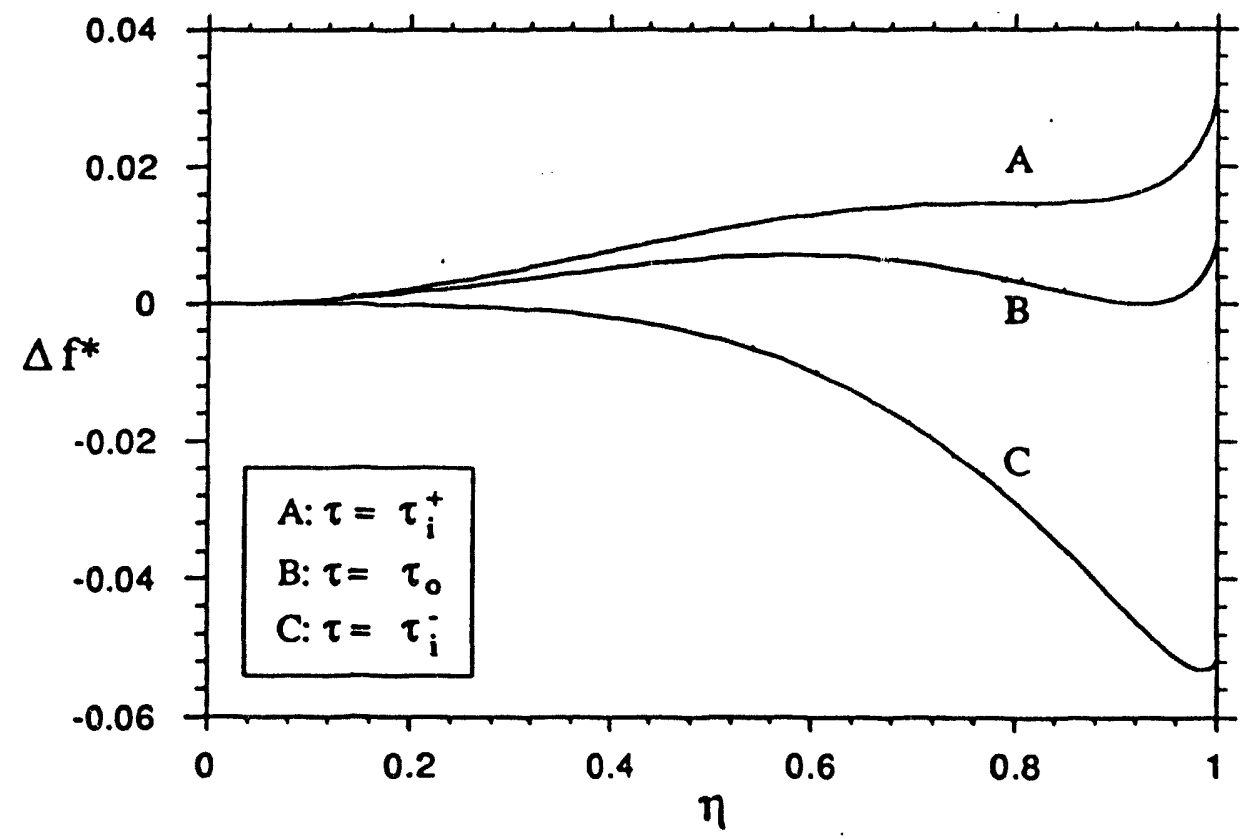

b)

Figure 3.3: (a) Temperature ( $\tau$ ) dependence of the long range order parameter $(\eta)$ for $c=0.5$ and $\alpha=0.1$. (b) Associated free energy diagrams ( $\Delta f$ vs. $\eta)$ for three different characteristic temperatures. 
Let us first discuss the temperatures labeled $\tau_{i}, \tau_{i}^{+}$and $\tau_{0}$ in Fig. 3.3a). By applying the mathematical definitions of $\tau_{i}, \tau_{i}^{+}$and $\tau_{0}$ (see Chapter 2) to the free energy expression given by eq. (3.40), the following analytic expressions can be derived:

$$
\begin{aligned}
& \tau_{i}=c(1-c) \\
& \tau_{i}^{+}=\tau_{i}^{+}+\frac{A_{4}{ }^{2}}{3 A A_{6}} \\
& \tau_{0}=\tau_{i}^{+}+\frac{A_{4}{ }^{2}}{4 A A_{6}}
\end{aligned}
$$

where $A=\frac{c^{2}}{2 \tau_{i}^{*}}=\frac{c}{2(1-c)}$.

It can be seen from the eq. (3.42) that $\tau_{i}$ is not influenced by $\alpha$ whereas $\tau_{i}^{\ddagger}$ displays a pronounced dependence on it. This is because $\tau_{i}^{*}$ is related to the stability of the disordered phase through the harmonic $\eta^{2}$ term in the free energy whereas $\tau_{i}^{+}$is related to the stability of the ordered phase which should be affected by the anharmonic $\eta^{4}$ term. From eq.'s (3.42b) and $(3.42 c)$, the width of the metastable disordered fcc phase region relative to that of the metastable ordered $\mathrm{LI}_{0}$ phase region is determined to be three times wider, irrespective of the relative magnitude of $\alpha$ (see Fig. 3.3a). The ratio (3:1) comes directly from the $\eta^{4}$ dependence of the strain energy term in the free energy.

\subsubsection{Threshold $\alpha\left(=\frac{e}{\left|V\left(k^{\circ}\right)\right|}\right)$ value}

The relative importance of the tetragonal lattice distortion in making the $L_{1}$ ordering transition first order is strongly dependent on $\alpha$. In fact, there exists a threshold value for the onset of the first order transition and it can be determined by analysis of the fourth order coefficient term given by eq. (3.41b). According to the expression, the first order transformation is only possible when the negative strain energy term accounting for the relaxation of the tetragonal distortion overrides the positive entropy term. In the mean field free energy model considered, the first order $L_{1}{ }_{0}$ ordering transition arises when the long 
range order parameter has branching solutions above $\tau_{i}$. Applying these conditions to eq.(3.41b) gives rise to the threshold value of $\alpha$ specified by eq. (3.43).

$$
\alpha_{\text {thresh }}=\frac{c(1-c)}{192}\left[\frac{1}{c^{3}}+\frac{1}{(1-c)^{3}}\right]
$$

For $c=0.5$, the equation yields $\alpha_{\text {thresh }}=0.02083$. From this analysis, it may be concluded that treating the tetragonal distortion in thermodynamic studies of the $\mathrm{LI}_{0}$ ordering transition is increasingly important when the values of $e$ and $\left|V\left(k^{\circ}\right)\right|$ give a ratio which is above the threshold value.

The direct relationship between $e$ and $\left|V\left(k^{\circ}\right)\right|$ which came with the use of the reduced free energy provides us an important guide for assessing the relative effect of the tetragonal distortion of the thermodynamic nature of a $\mathrm{L1}_{0}$ ordering transition amohg different alloy systems. Assumming $e$ has a substantial magnitude, the effect may be significant for such a system whose transition temperature for the $L 1_{0}$ ordering is relatively low since $\left|V\left(k^{\circ}\right)\right|$ is expected to be smaller in such a case. Conversely, for an alloy with a high $\mathrm{L}_{0}$ ordering temperature, a large tetragonality alone may not bring about the first order nature of the transition. This is because the relative importance of the femperature dependent entropic contribution in competition with the strain energy term may vary among systems with different ordering transition temperatures. In this regard, binary alloys such as CuAu $\left(T_{c}=385^{\circ} \mathrm{C}\right)$ and InMg $\left(T_{c}=330^{\circ} \mathrm{C}\right)$ may be taken as the possible systems where the signfficant effect of the tetragonal distortion on the thermodynamics of the ordering is expected. We will give more detailed considerations on these systems in section 3.3.3.

The criterion given above states that the mean field approximation is inadequate to treat such an $L 1_{0}$ system with an $\alpha$ below the threshold value. This result, however, should not be taken as a suggestion that neglect of the tetragonal distortion may be justified when they are treated in terms of the higher order approximations. This is because the same magnitude of the strain energy term has a more pronounced effect in the higher order approximations 
which produce systematically reduced entropy terms relative to the exaggerated mean field entropy.

\subsubsection{Two Phase $\left(f c c+L 1_{0}\right)$ Equllibrium.}

The qualitative nature of the phase equilibrium between the disordered fcc and the $\mathrm{LI}_{0}$ ordered phases has been studied by determining numerically the phase boundaries for various values of $\alpha$ and $\omega\left(=V(0) /\left|V\left(k^{\circ}\right)\right|\right)$. In this part of the calculation, no $L_{1}$ phase is allowed to form in order to see the properties of the free energy given by eq. (3.34) more clearly. In fact, the situation we are treating is not hypothetical but has reference to the cases of several L1, ordering alloys: as a typical example, NiPt alloy ${ }^{[74]}\left(\mathrm{NiPt}_{3} \mathrm{LI}_{2}\right.$ phase is not existing). The $\omega$ values were arbitrarily chosen with the presumption that the $\mathrm{LI}_{0}$ ordered structure is the stable ground state structure. Summary of the calculations are presented in Fig. 3.4a) and 3.4b).

Curve $\mathrm{C}$ in Fig. 3.4a) represents a typical phase boundary predicted by the rigid lattice mean field model. In agreement with the curve $A$ in Fig. 3.2, it separates the L $1_{0}$ phase from the fcc phase by a line of the second order phase transitions. As shown by curves $A$ and $B$ in Fig. 3.4a), phase diagrams of fundamentally different characteristics are predicted when the tetragonal distortion of the lattice is taken into account. A significant increase in the stability of the L1 $1_{0}$ ordered phase as well as the existence of a two phase field is noticeable, compared to the rigid lattice phase diagram. The relative width of the two phase field is found to be strongly dependent not only on $\alpha$ but also on $\omega$ which effectively measures the decomposition tendency of an undercooled off-stoichiometric fcc disordered phase. This is shown in Fig. 3.4b). The phase diagram attained with $\omega=1$ and $\alpha=0.05$ is instructive and is replotted in Fig. 3.5a) together with the tree energy-composition diagrams (3.5b) to $3.5 \mathrm{~d}$ ) where $f^{\circ}=f^{*}-\mu c, \mu$ : chemical potential) for the three selected temperatures. As the phase diagram shows, when the decomposition tendency is weak ( $\omega$ is large), the two phase field boundaries merge into a second order transition line at a tricritical point $\left(\tau_{\mathrm{B}}\right)$ where the 


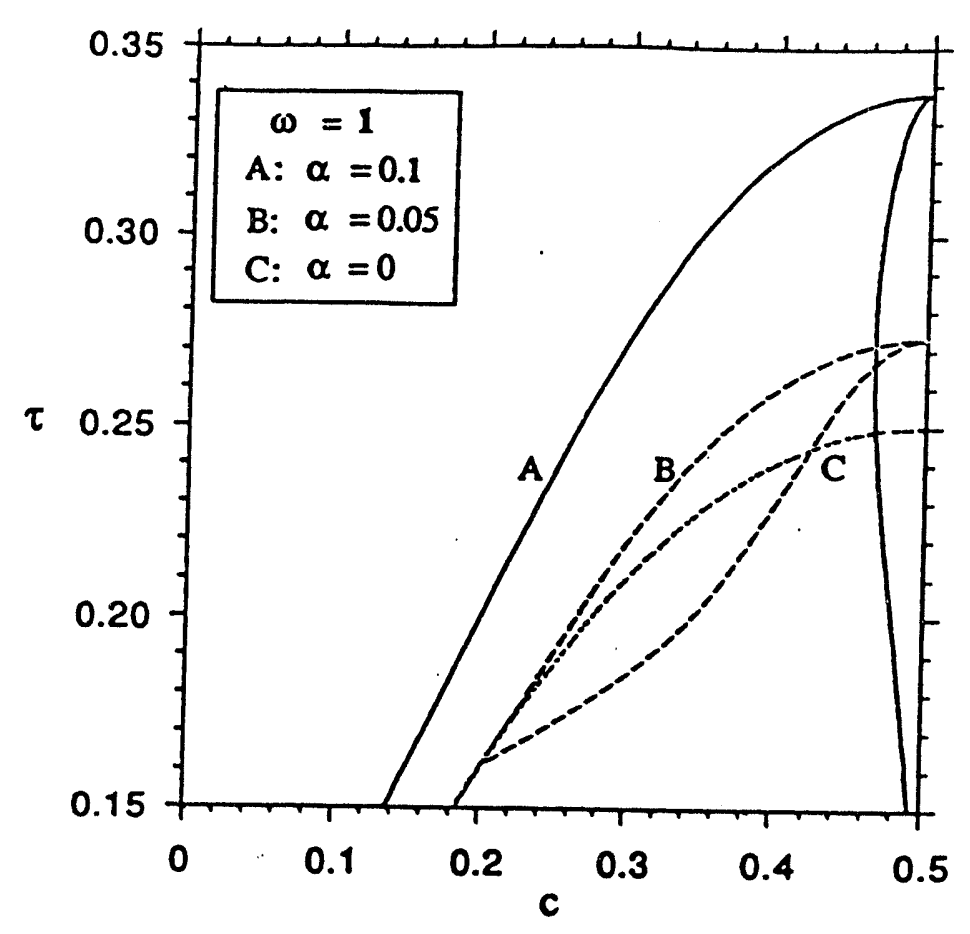

a)

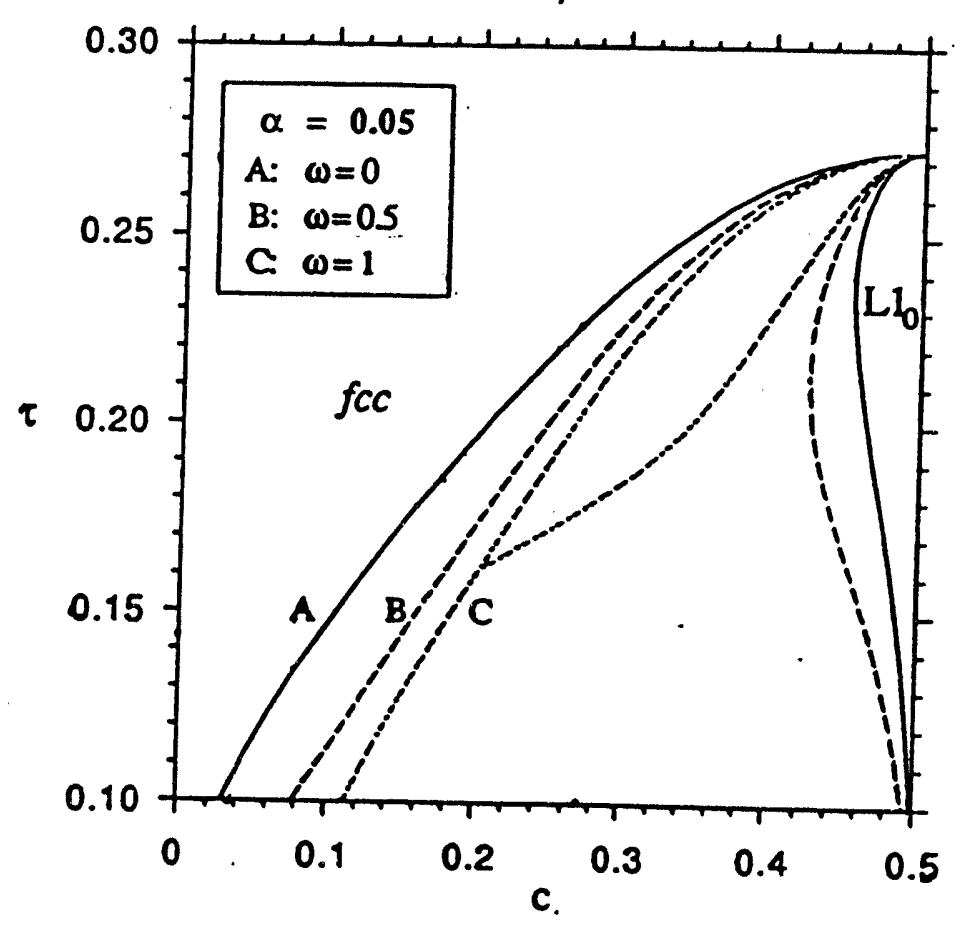

b)

Figure 3.4: Calculated phase diagrams for various $\alpha$ and $\omega$ values. No $L 1_{2}$ phase is allowed in these phase diagrams. (a) $\omega=1$. (b) $\alpha=0.05$. From (a), notice that, consistent with the results shown in Figure 3.2, phase diagram characteristics change drastically as $\alpha$ increases from $\alpha=0$ (rigid state). 


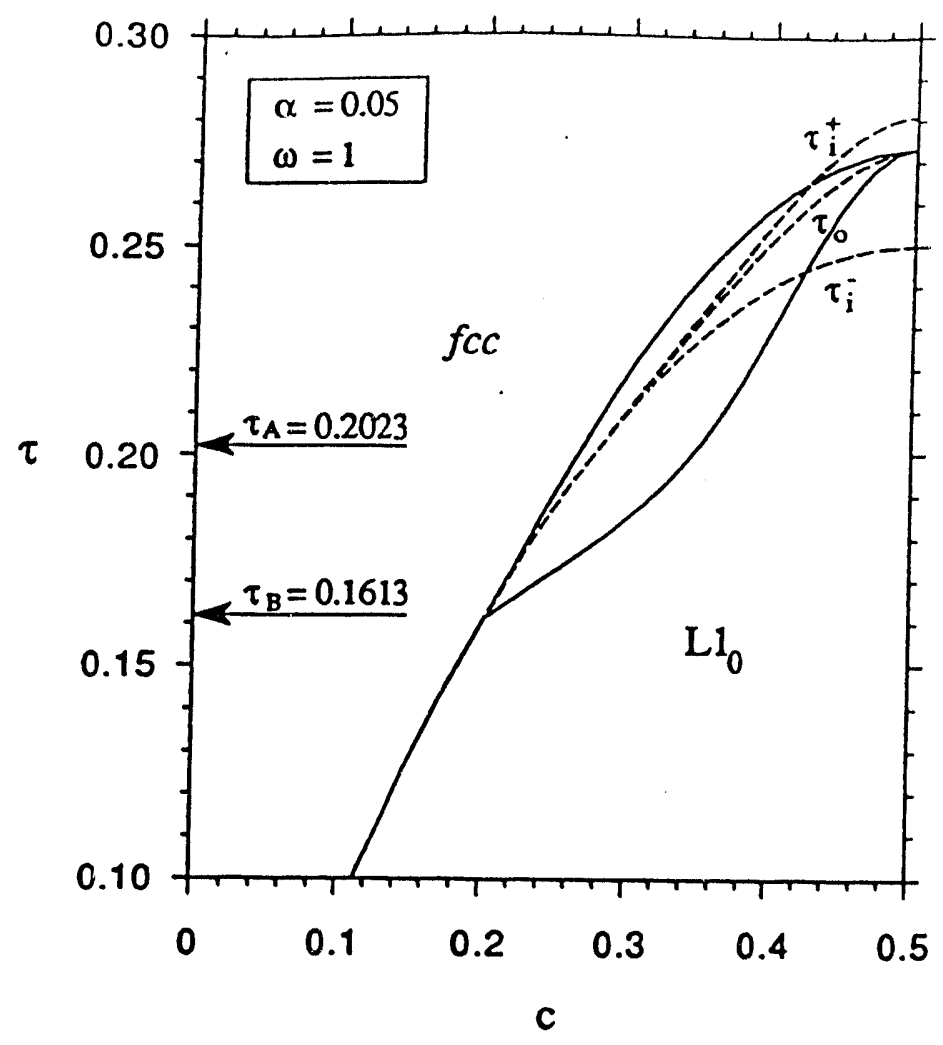

a)

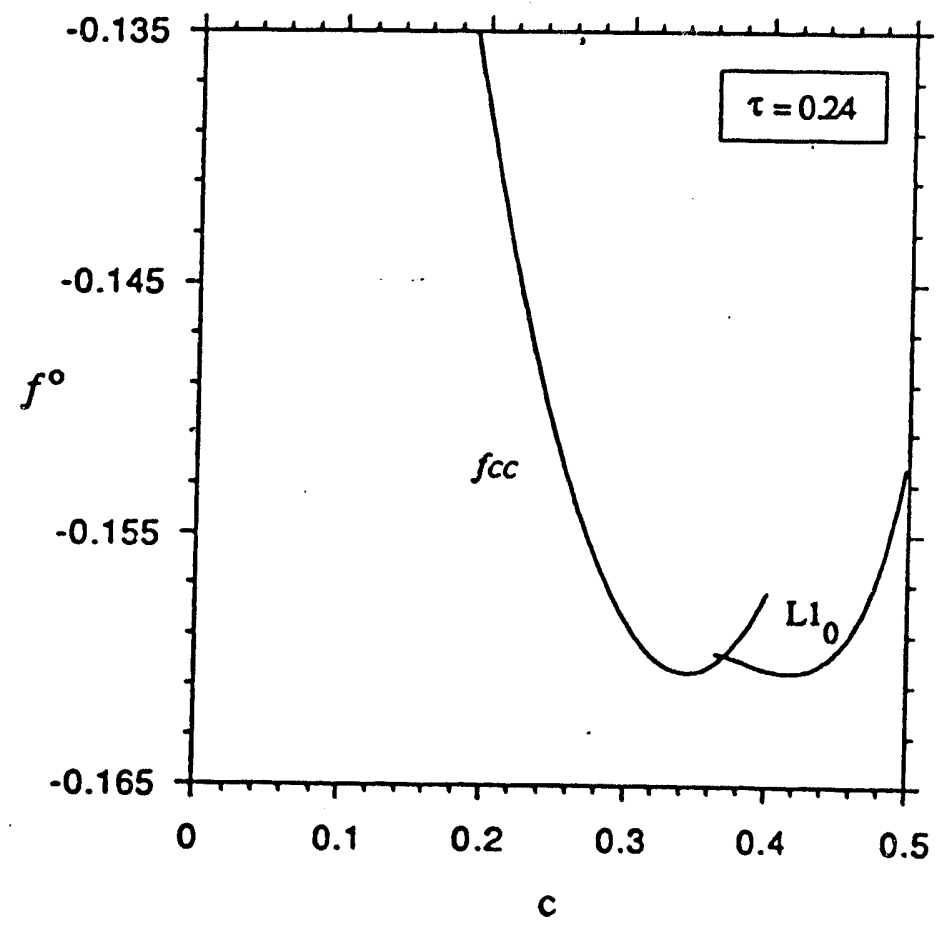

b)

Figure 3.5: (a) Phase diagram $C(\alpha=0.05$ and $\omega=1)$ of Figure 3.4 with additional thermodynamic information. Under the diminishing influence of the strain energy term, lines of three characteristic temperatures merge into a single line at $\tau=\tau_{A}$ and two phase boundaries merge into a second order transition line at $\tau=\tau_{B}$. $(b, c$, and d) Associated free energy-composition diagrams for the three specified temperatures. 


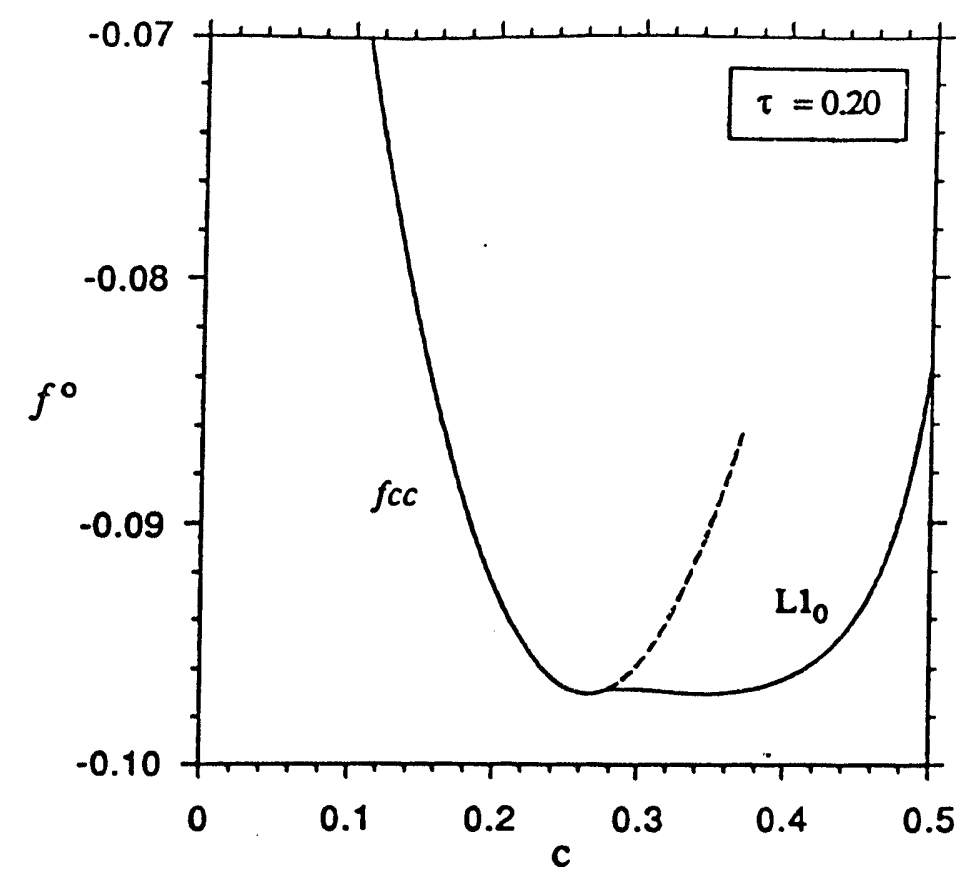

c)

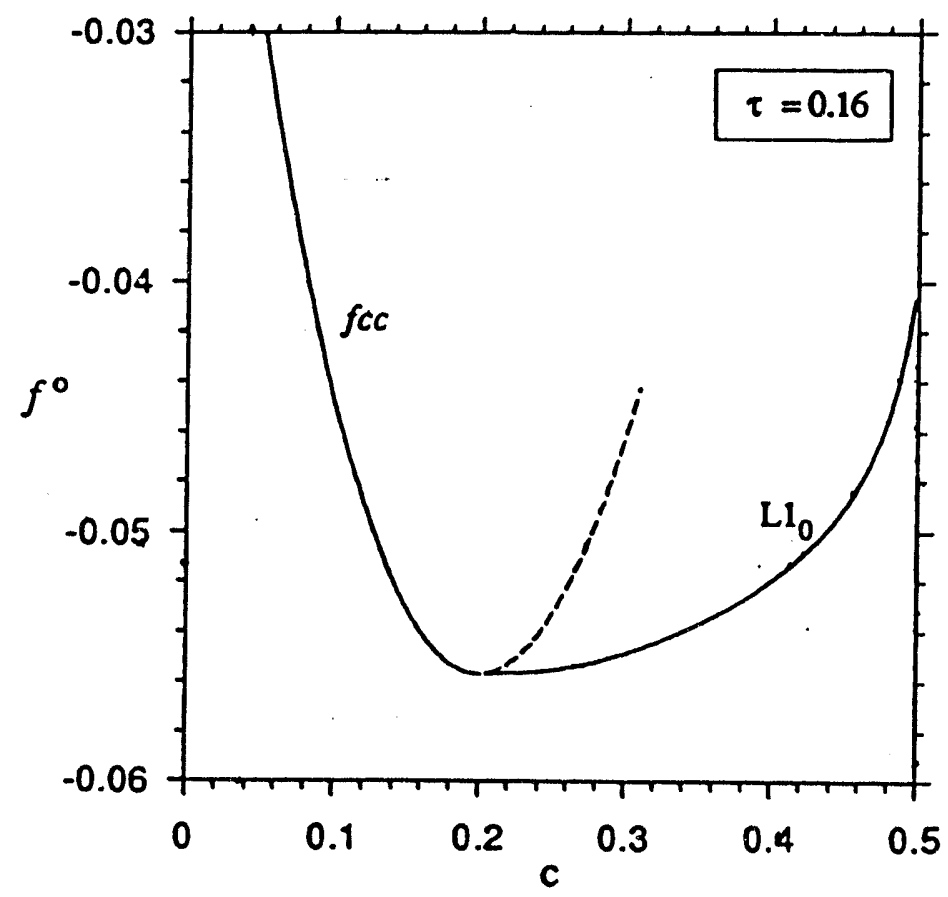

d)

Figure 3.5: cont. When $\tau<\tau_{\lambda}$, foc phase is unstable in the composition range where the free energy of fcc phase is larger than that of $L 1_{0}$ phase. This is shown in dotted lines in (c) and (d). 
coefficient of the harmonic term as well as that of the fourth order term vanishes, i.e. $A_{2}=A_{4}$ $=0$ in eq. (3.40). Within the context of the free energy model used, in the composition range where the fcc to $\mathrm{L1}_{0}$ ordering is of second order, the homogeneous ordered phase may phase-separate into the two phase mixture of $\left(f c c+L 1_{0}\right)$ by secondary decomposition. In principle, the feature shown in Fig. 3.5 (a) is not physically implausible. Nevertheless, in view of the intrinsic deficiency of the mean field approximation, i.e. neglect of the correlation effects, we presume that it may be rather an artifact due to the diminishing influence of the strain energy term in the solvent rich regions. It should be noted that the effect of the strain energy term is, however, still conspicuous in the solute rich regions $(c>0.25)$ where the two phase $\left(f c c+L 1_{0}\right)$ equilibrium may be only physically meaningful if the $L_{2}$ phase is allowed. For the $\omega$ values tested (up to $\omega=4$ ), the existence of a two phase region has been always predicted for the composition range concemed. This derives from the fact that $\tau_{\mathrm{A}}$ (where $A_{4}$ $=0$ ) is solely determined by $\alpha$ and, therefore, can not be lower than $\tau_{B}$ irrespective of $\omega$.

\subsubsection{Phase Dlagram of the $<001>^{*} f c c$ Speclal Polnt Ordered Structures.}

Calculation of the phase diagram involving $f c c, L 1_{2}$ and $L 1_{0}$ phases was made using a model binary system with $\alpha=0.05$ and $\omega=0.5$. The resulting phase diagram is shown in Fig. 3.6 (b) together with the corresponding rigid lattice phase diagram (3.6 (a)). Some topological features are quite distinct in the phase diagram of Fig. 3.6 (b), compared to that of Fig. 3.6 (a).

1) the disappearance of the two second order transitions (fcc to $L 1_{2}$ and fcc to $L 1_{0}$ ) at the equiatomic composition.

2) the appearance of the $\left(f c c+L 1_{0}\right)$ two phase field.

3) the appearance of an invariant peritectoid reaction at an intermediate temperature.

These main features are unique to the mean-field free energy model considered and do not appear to deperid on the particular choice of $\alpha$ and $\omega$ values. 


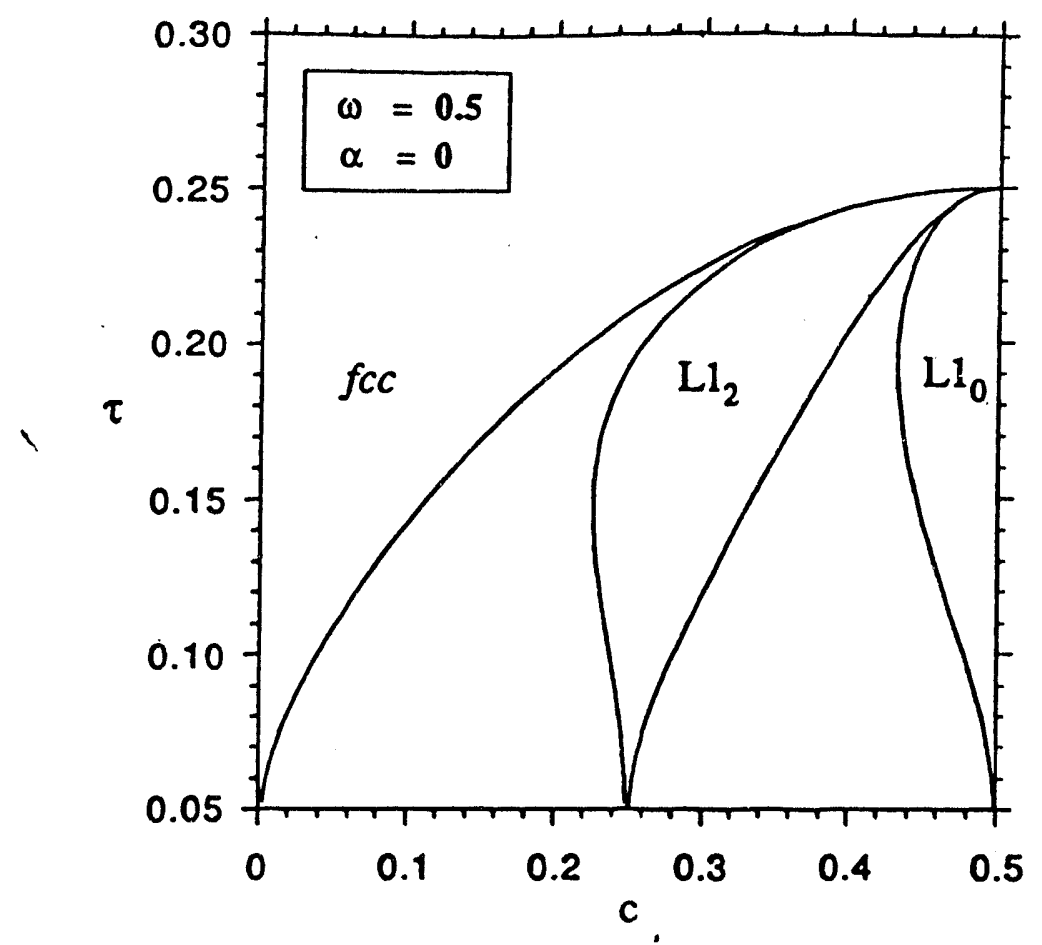

a)

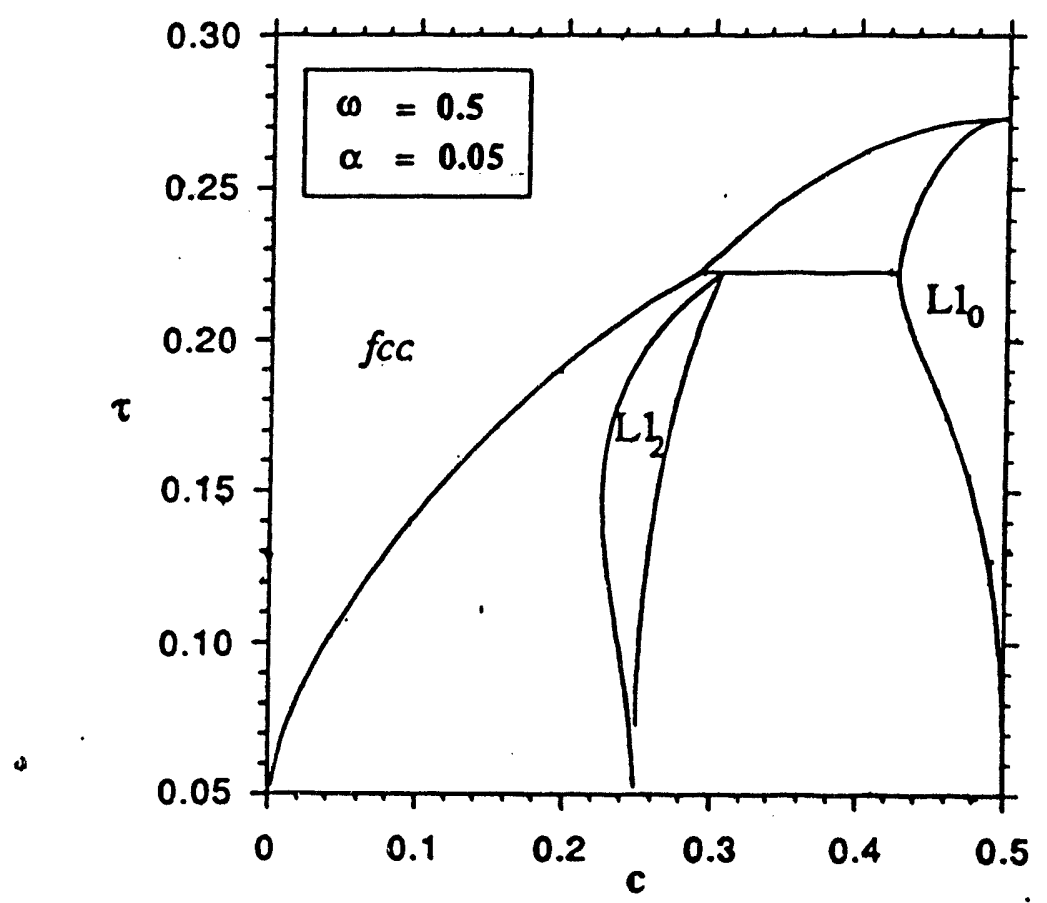

b)

Figure 3.6: Calculated phase diagrams of $<001\rangle^{\circ}$ special point ordered structures: (a) with no lattice relaxation and (b) with relaxation of configuration-dependent elastic energy contributions. 
In our earlier discussion, we proposed that the effect of a tetragonal lattice distortion would be more pronounced in alloy systems which have relatively low fcc to $L 1_{0}$ ordering transition temperatures. In this regard, it is quite interesting that the calculated phase diagram displays some essential features of experimental phase diagrams of certain binary alloy systems such as CuAu and InMg alloys. Experimental phase diagrams of these alloys [75.76] are presented in Fig. 3.7. A significant topological analogy between these phase diagrams and the calculated phase diagram is readily noticed from the $\mathrm{AuCu}-\mathrm{Au}_{3} \mathrm{Cu}$ (if the presence of CuAull phase is neglected) and the $\operatorname{lnMg}-\ln 3 \mathrm{Mg}$ side of the respective phase diagrams.

Due to the simplicity of the model used, however, care should be taken in evaluating such an agreement. This is because the tetragonal lattice distortion, in principle, can only explain the features directly related to the increase in the stability of the $L_{1}$ phase. The absence of the congruent point at the stoichiometric $L 1_{2}$ phase composition and the appearance of a peritectoid reaction are, in fact, inherited from the attributes of the mean field model. A more basic account of this feature may need to involve considerations of the nature of atomic interactions. With regard to this, some interesting results are found from previous investigations.

According to the CVM [7] and the Monte Carlo calculations $[8,9]$ of the phase diagrams for the rigid fcc lattice Ising model with first $\left(V_{1}\right)$ and second $\left(V_{2}\right)$ nearest neighbor interaction, when $V_{2} / V_{1}$ is less than -0.25 , a phase diagram is predicted which has a topology identical to that of the rigid lattice mean field phase diagram (Fig. 3.6(a)). In the case of CuAu alloys, analyses of the $X$-ray diffuse scattering data from disordered $\mathrm{CuAu}$ and $\mathrm{CuAu}_{3}$ alloys have yielded $V_{2} / V_{1}$ KuAu $=-0.4$ and $V_{2} / V_{1} K_{U A u_{3}} \equiv-0.53$ [77]t. This suggests that the mean field type feature shown in Fig. 3.6 (a) should be expected in the Au rich CuAu region when the rigid lattice Ising model is applied to calculate the phase diagram, if pairwise interaction and the composition independence of the interatomic pair potential are assummed. Although

\footnotetext{
The values were taken from the data for $800^{\circ} \mathrm{K}$ (CuAu) and for $573^{\circ} \mathrm{K}\left(\mathrm{CuAu}_{3}\right)$.
} 


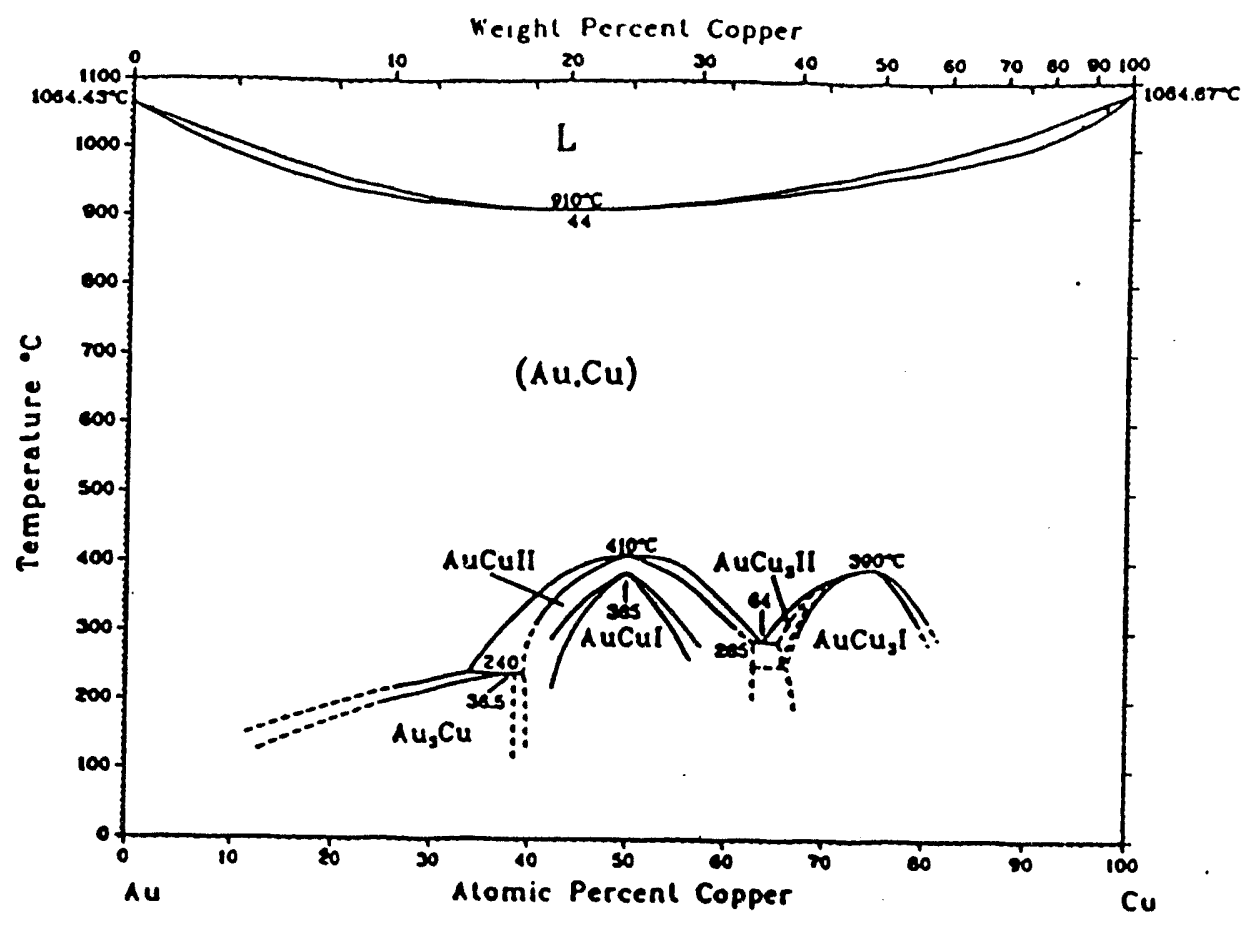

H. Okamoto. D.J. Chakrabarti. O.E. Laughtin. and T.6. Massalskı. 1987

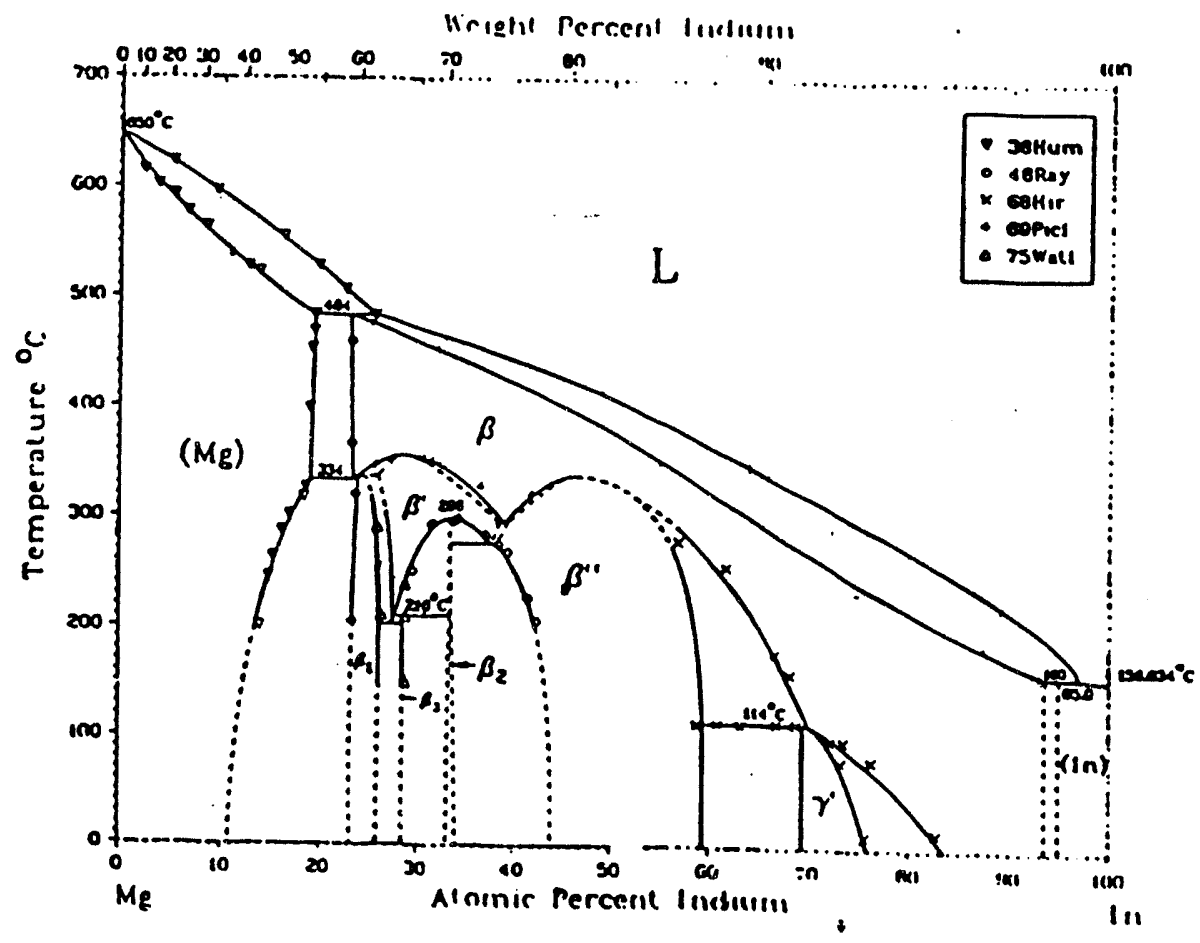

A.A. Nayeb.Hashemi and J. B. Clark. 1985.

Figure 3.7: Experimental phase diagrams of the CuAu and the InMg alloy systems: taken from [75] and [76], respectively. 
relevant experimental data are not available, the nature of atomic interactions is expected to be similar for the InMg alloys as well.

Based on this understanding, we have recalculated the phase diagram of Fig. $3.6(\mathrm{~b})$ for the CuAu alloy. From the room temperature data of the lattice parameters ${ }^{[72]}$ and of the elastic constants [78], the value of $e$ (eq. (3.37)) was determined to be $e / k=91.4^{\circ} \mathrm{K}$ where $k$ is the Boltzman constant. The chemical pairwise interaction energy $V\left(k^{\circ}\right)$ of the equiatomic CuAu alloy is not available in the literature and use was made of an estimated $V\left(k^{\circ}\right)$ value $\left(V\left(\mathrm{k}^{\circ}\right) / \mathrm{k}=-2361^{\circ} \mathrm{K}\right)$ of the $\mathrm{CuAu}_{3}$ alloy with the assumption that it does not change with composition: the estimation was made through $V\left(k^{\circ}\right)=\sum_{r} V(r) e^{-i k \cdot 1}$ by use of the real space interatomic potential values of up to the 8th coordination shell determined by Bessiere et al. from $X$-ray diffuse scattering of the disordered alloy quenched from $T=573^{\circ} \mathrm{K}$ [79]. The $V(0) / V\left(k^{\circ}\right)$ ratio $(=3.375)$ was determined by use of the $V_{i} N_{1}(i: i$ th coordination shell) values of the equiatomic CuAu alloy due to Metcalfe and Leake [77]. The resulting phase diagram is presented in Fig. 3.8. In association with the assumption used, that is, composition independence of $V\left(k^{\circ}\right)$, the calculated diagram clearly suggests that the tetragonal distortion of the $L 1_{0}$ ordered phase may be a contribution of substantial importance to engender the feature of interest in the CuAu phase diagram.

Within the framework of the mean field theory, the anharmonic fourth order term accounting for the relaxation of the tetragonal distortion may be regarded effectively as the internal energy correction due to the four body interaction of strength $\alpha$ which tries to form a Au or $\mathrm{Cu}$ single atomic layer. That is,

$$
\Delta U=-\alpha\left\langle\delta p\left(r_{1}\right) \delta p\left(r_{2}\right) \delta p\left(r_{3}\right) \delta p\left(r_{4}\right)\right\rangle \sim-\alpha\left\langle\delta p\left(r_{1}\right) X \delta p\left(r_{2}\right) X \delta p\left(r_{3}\right) X \delta p\left(r_{4}\right)\right\rangle=-\alpha \eta^{4}
$$

This is, in fact, in interesting contrast to the prediction from a previous work. After Van Baar's initial work [4], Kikuchi and deFontaine [5] have calculated the CuAu phase diagram in the CVM tetrahedron approximation to the first nearest neighbor Ising model by taking aciount of the tetrahedron CuCuCuAu and AuAuAuCu four body interactions. Many body interactions 


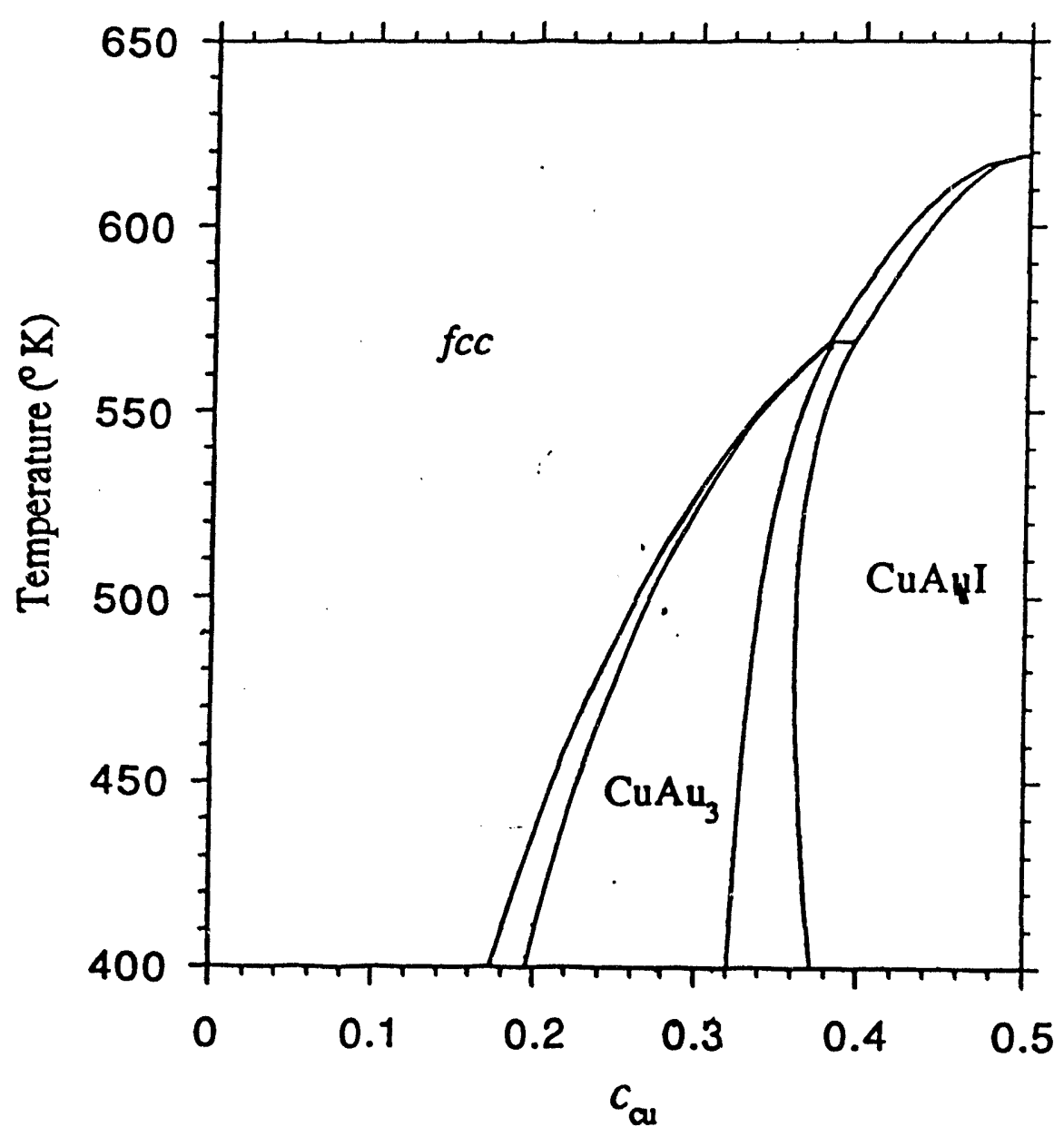

iI
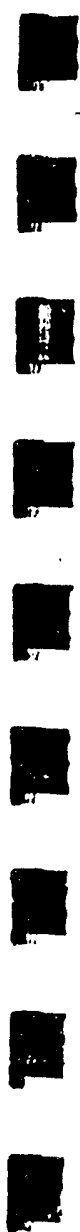

Figure 3.8: The CuAu-CuAus side of the CuAu phase diagram calculated by the mean fleld theory incorporating the tetragonal lattice distortion of the L1, phase. 
thus introduced have resulted in the features which show a good agreement with those of the experimental phase diagram including some quantitative aspects. It should be noted, however, that their calculated phase diagram is a rigid lattice diagram in view of the rigid coherent state of the $\mathrm{LI}_{0}$ phase. Accordingly, the parameters used to fit their diagram into the experimental phase diagram may not be of much physical meaning.

Although our calculated phase diagram successfully reproduces the topological features of the Au-rich side of the CuAu phase diagram, it fails to reproduce quantitative aspects of the diagram. Except for the assummed composition independence of $V\left(k^{0}\right)$, this is mainly due to an intrinsic deficiency of the mean field model, that is, the neglect of correlation effects. Calculation of a reliable phase diagram and thus a precise assessment of the role of the tetragonal lattice distortion should be then made by use of a free energy model which incorporates the correlation effects as well as the tetragonal lattice distortion. Within the framework of the SCW model, correlation effects can be effectively taken into account by the method developed by Badalyan and Khachaturyan [80].

\subsection{Conclusion}

A theoretical investigation was carried out to understand the effects of the tetragonal lattice distortion of the $L 1_{0}$ phase on the thermodynamics of the $L 1_{0}$ ordering and on the alloy phase equilibria involving the $L 1_{0}$ phase. In order to obtain a free energy model which will serve these purposes, we have treated the problem of rigidity of the Ising model. The treatment concerns itself purely with removal of the elastic energy contributions from a rigid lattice free energy which prevent the relaxation of the strain free rigid lattice into the stress free one. A generalized formulation was presented in a form which can be utilized directly to study incoherent two phase equilibria. By employing the SCW mean field model as a rigid lattice free energy model, we have applied the formulation to the case of interest. 
We have shown clearly that the thermodynamic stability of the $L 1_{0}$ phase is influenced by the relaxation of the tetragonal distortion, through competition between the associated negative strain energy and the positive entropic contribution. In association with this, a criterion was established. It states that within the context of the mean field free energy model, the first order nature of the $L 1_{0}$ ordering is recovered when $\alpha\left(\frac{e}{\overline{V\left(k^{\circ}\right)}}\right)$ exceeds a threshold value $(=0.02083$ ). From this criterion, a conclusion was reached that the problem of a rigid lattice free energy model and thus of the neglect of the tetragonal distortion, could be more serious for alloy systems with lower L1 $1_{0}$ ordering transition temperatures (e.g. CuAu and $(\mathrm{nMg})$, given the same magnitude of the tetragonal distortion. By taking into account the tetragonal distortion, we have calculated a prototype phase diagram of the $<001\rangle^{*}$ special point ordered structures. The resulting phase diagram has displayed fundamentally different characteristics, as compared to the one due to a rigid lattice mean field model. In fact, the topological features of the calculated phase diagram was found to resemble those of the $\mathrm{CuAu}_{\mathrm{CuAu}}$ and the $\mathrm{InMg} \cdot \mathrm{I} \mathrm{n}_{3} \mathrm{Mg}$ side of the respective experimental phase diagrams. Such an agreement clearly suggests an important role of the tetragonal distortion, as opposed to that of many body interaction or equivalently of the composition-dependent interatomic potential which are proposed to explain the same features in the existing theoretical work. The conclusions obtained in this study are qualitative and, therefore, a precise assessment of the role of tetragonal distortion snould be made by use of a free energy model which takes into account correlation effects. It is easily expected that the effects of the tetragonal distortion would be more pronounced in such a free energy model since it will produce a reduced entropy term relative to the exaggerated mean field entropy. 


\section{Effects of Tetragonal Distortion on the Morphology of L10 Ordered Microstructure.}

In this chapter, we examine the $L_{0}$ ordering of an $\mathrm{Fe}-45$ at.\%Pd alloy in an attempt to better understand the effects of tetragonal lattice distortion on the morphology of $\mathrm{L}_{0}$ ordered microstructure. In section 4.2, we investigate various ordered states with a particular attention to the morphology of the single $L 1_{0}$ phase microstructure. Based on experimental findings, in section 4.3, we discuss some of the essential features of the $\mathrm{LI}_{0}$ microstructure such as the variant habit plane, the variant volume fraction and the shape of a variant plate within the framework of elasticity theories of polytwin morphology and of a coherent plate-like inclusion. This will show us to what extent the theories may be utilized to understand the microstructure of the single $L 1_{0}$ phase.

\subsection{Experimental Procedure}

As-cast buttons of the Fe-45.1 at.\% Pd alloy were provided by the Lawrence Livermore National Laboratory. The buttons were initially given a homogenization treatment of 3 days at $1150^{\circ} \mathrm{C}$. Subsequently, they were sliced into pieces with thicknesses of about $0.5 \mathrm{~mm}$ and were cold-rolled to a thickness of about $0.2 \mathrm{~mm}$. After being subject to an intermediate anneal of $10 \mathrm{~min}$. at $1150^{\circ} \mathrm{C}$, the specimens were finally cold-rolled into sheets with thicknesses of less than $0.1 \mathrm{~mm}$. The sheet specimens were then given a disordering treatment for $15 \mathrm{~min}$. at $1150^{\circ} \mathrm{C}$. For each heat treatment discussed so far, the specimens were encapsulated in argon back-filled quartz tubes and were heated in a box furnace which was maintained at a temperature controlled to an accuracy of $\pm 3^{\circ} \mathrm{C}$. Quenching of the 
disordered specimens was carried out by rapidly transferring the quartz tubes to iced-brine water and smashing them onto a heavy metal object which was immersed in the quenchant.

Aging of the quenched specimens was performed either in a salt bath for short time aging (< $30 \mathrm{~min}$. ) or in a three zone tube furnace for longer aging treatments. For fumace aging, the specimens were encapsulated in argon back-filled pyrex tubes. Aged specimens were washed in hydrochloric acid diluted with water. For the TEM study, discs of $3 \mathrm{~mm}$ in dia. were prepared from the aged specimens and were electro-polished using a twin-jet electropolisher with an electrolyte of 82 vol.\% acetic acid, 9 vol.\% perchloric acid and 9 vol.\% ethanol at a temperature of $3^{\circ} \mathrm{C}$ and a potential of $17 \mathrm{~V}$. Electron microscopy was conducted using a Philips 420T microscope as well as a JEOL $120 \mathrm{CX}$, both operating at $120 \mathrm{keV}$.

\subsection{Experimental Results}

\subsubsection{As-Quenched State.}

The overall characteristics of the as-quenched state are shown in Fig. 4.1. From the Selected Area Diftraction Patterns (SADP) of Fig. 4.1a) and 4.1c), it is noted that the superlattice reflections are composed of very faint diffuse intensity (indicated by arrows). Since the electron scattering powers of Fe and Pd atoms are significantly different [81], the faint superlattice intensity may be taken as an indication that the $L 1_{0}$ ordering of the disordered fcc phase is nearly suppressed during the quenching process. In this regard, interesting features are the fairly strong diffuse streaks in the SADP's (along the <011>* directions in (a) and the <1.12 $>^{\circ}$ directions in (c) ${ }^{\dagger}$ ) and the noticeable mottled strain contrast (yet weakly directional i.e. faint tweed) in the Bright Field (BF) images.

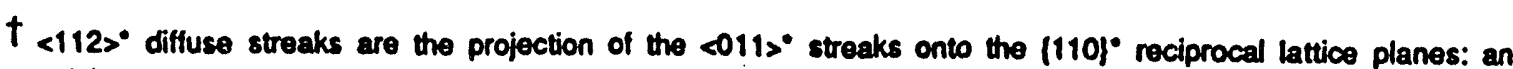
astorisk (") denotos a reciprocal space. 

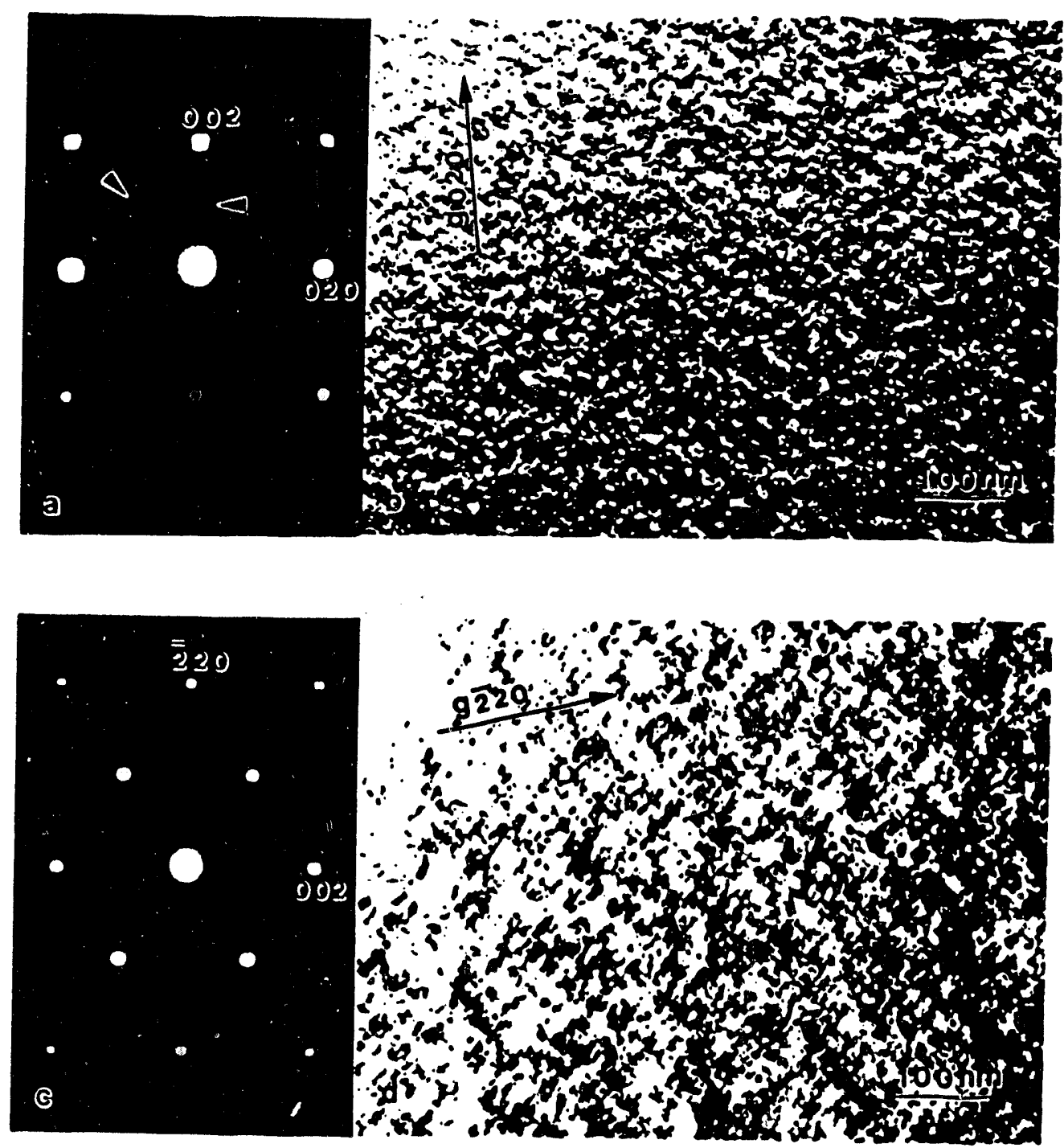

Figure 4.1: The as-quenched state of an Fe-45at.\%Pd alloy. (a) [100] zone axis SADP. (b) Two-beam BF image: $g=020$. (c) [110] zone axis SADP. (d) Two-beam BF image: $g=\overline{2} 20$. Notice a very faint intensity of the superlattice reflections, suggesting the existence of the short range order clusters of the L1, phase. This, together with a high elastic anisotropy of the Fe-Pd alloy, is responsible for the fairly strong diffuse streaks in (a) and (c) and the noticeable mottled (yet faint tweed) strain contrast in (b) and (d). 
For Fe-Pd alloys, the diffuse streaks and the associated strain contrast (particularly the tweed contrast) have been studied in the incipient stages of the foc to $L 1_{0}$ ordering of an equiatomic alloy $[15,18]$ as well as in premartensitic states of the fcc to fct thermoelastic martensite transformation of alloys of near 30 at.\% Pd. [15-19] In each case, it was suggested that the features are due to an elastic shear distortion (or $\{011\}<01 \overline{1}\rangle$ static atomic displacement) of the matrix fcc phase, induced by the existence of coherent tetragonal distortion centers either replacive or displacive in nature. For the present alloy, we do not regard the observed feaiures as a premartensitic effect signalling the onset of the fcc to fct thurmoelastic martensitic transformation since this transformation would not occur even at 0 o $K$ when the Pd content is in excess of $\sim 32$ at.\%Pd, according to the martensite phase diagram constructed by Sugiyama et al. $[15,16]$. Accordingly, it follows that the tetragonal distortion centers which are accountable for the observed features may be of replacive nature, that is, short range order clusters of the $L^{\prime} y_{0}$ phase, consistent with the faint diffuse intensity of the superlattice reflections.

The appearance of the strain contrast 'e associated diffuse streaks requires not only a perturbing strain field producing it $>$ static atomic displacement but also a small resistance 10 such a displacement, as characterized by a high elastic anisotropy ratio $A$ $\left(=2 C_{44} /\left(C_{11}-C_{12}\right)\right)$. ${ }^{10,82,83]}$ According to the inelastic neutron scattering measurement made by Sato et al. [84], the anisotropy ratio of a disordered Fe-37 at.\%Pd alloy is about 3.8 at 295 ' $K$. We expect that the $A$ value of a disordered Fe-45 at.\%Pd alloy would be similar. This value is slightly higher than the value of $\mathrm{Cu}$ (3.2) which was invoked to explain the strong tweed contrast in early stages of precipitation in $\mathrm{Cu}$ rich $\mathrm{Cu}-\mathrm{Be}$ alloys [82]. From this, we conclude that the observed features of the as-quenched state may be ascribed to the combined effect of two factors: a weak strain field due to short range order clusters of the $L_{0}$ phase and a high elastic anisotropy ratio which promoted the $\{011\}<01 \overline{1}>$ shear distortion of the matrix fcc phase. 


\subsubsection{Aged States,}

\subsubsection{Development of Microstructure and Varlant Volume Fraction}

The quenched disordered alloys, as characterized above, were aged at $450{ }^{\circ} \mathrm{C}$ for various times. A set of TEM photographs taken from the alloy aged for 2 min. are shown in Fig. 4.2. From the BF image as well as from the SADP, one can notice that characteristic features remain mostly similar to those of the as-quenched state. Although superlattice reflections are now readily detectable, they are still quite broad and diffuse. Consistent with this, the Superlattice Dark Field (SDF) image taken with $g=001$ (Fig. 4.2c)) shows small ordered domains of sizes about $10-30 \AA$ which are more or less randomly dispersed throughout the matrix.

Slightly longer aging of the quenched alloy has produced features conspicuously different from those of the $2 \mathrm{~min}$. aged state. This is shown in Fig. 4.3, taken from the 5 min. aged state. From the SADP, it is found that the superlattice reflections are now much stronger and sharper and that the fundamental reflections display noticeable splitting (marked by an arrow in the SADP). This indicates that the tetragonal lattice distortion of the L1 $1_{0}$ ordered domains has increased with enhancement in long range order. One can also notice the sharper <011>* streaks in the SADP and the more pronounced tweed contrast in the BF image. In conjunction with these features, the SDF image shows regular arrays of the growing ordered domains along the $<011>$ fce directions, as opposed to a more or less random dispersion of the $2 \mathrm{~min}$. aged state. The <011> alignment of particles and the resulting strong tweed contrast in the BF images have been observed in precipitation reactions [82] as well as in the ordering reactions involving cubic to tetragonal symmetry change [18,85]. A theoretical account of the phenomenon was given by Fillinhham, Leamy and Tanner ${ }^{[86]}$ and by Wen, Khachaturyan and Morris ${ }^{[87}$ through computer simulation of the tweed misrostructure. It was shown that the alignment is a manifestation of the elastic energy minimization, resulting from the strain-induced elastic interaction among tetragonal 


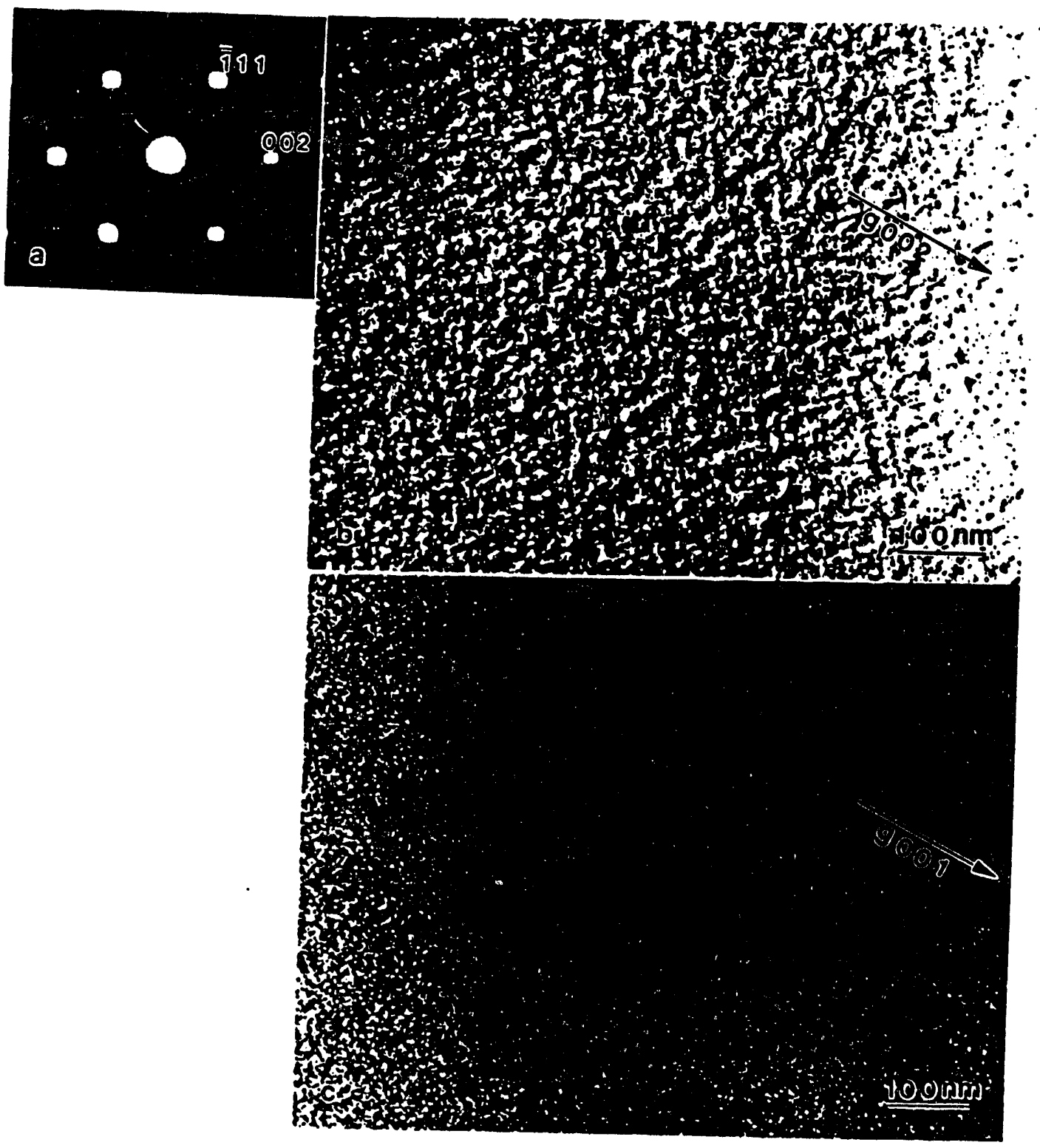

Figure 4.2: Alloy aged at $450^{\circ} \mathrm{C}$ for $2 \mathrm{~min}$. (a) [110] zone axis SADP. (b) Two-beam BF image taken with $g=002$. (c) SDF image taken with $g=001$. Along with the detectable, yet diffuse intensity of the superlattice reflections in (a), notice from (c) small ordered domains which are more or less randomly distributed throughout the matrix. 


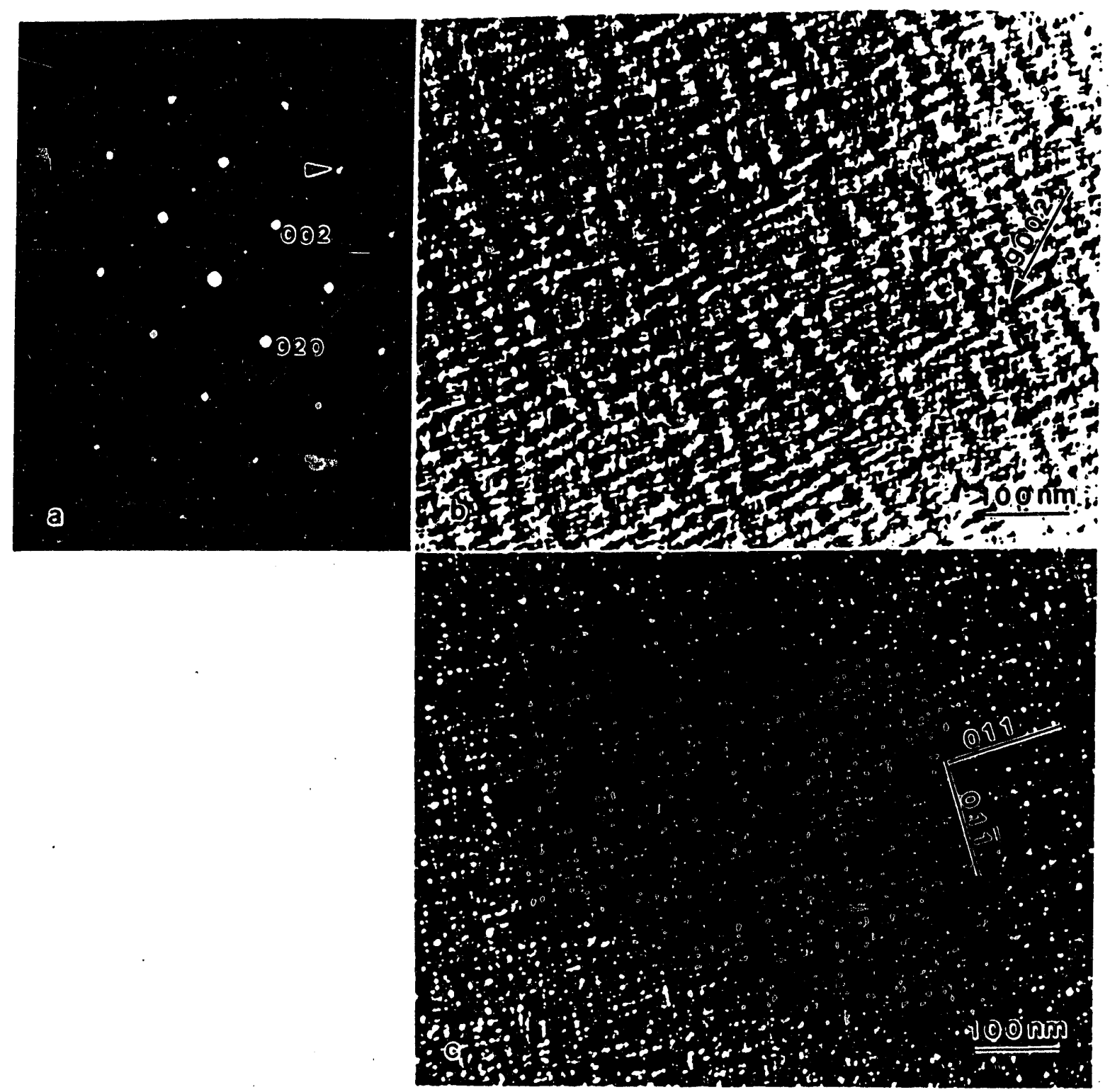

Figure 4.3: Alloy aged at $450^{\circ} \mathrm{C}$ for $5 \mathrm{~min}$. (a) [100] zone axis SADP. (b) Two-beam BF image taken with $\mathbf{g}=002$. (c) SDF image taken with $\mathbf{g}=001$. In co.junction with the <011> streaks in (a) and the pronounced tweed contrast in (b), notice from (c) regular arrays of the ordered domains along the $<011>$ directions. This alignment of particles is accompanied by a change in relative volume fraction among different crystallographic variants, as shown by the non-uniform distribution of the intensity of superlattice reflections in (a). 
strain centers. An interesting feature in the SADP regarding the constitution of the tweed microstructure is the non-uniform distribution of the intensity of superlattice reflections among 3 different crystallographic variants. This suggests that the spatial rearrangement of the ordered domains into the regular arrays is accompanied by a change in the relative volume fraction of different variants.

With further aging, ordering domains grow and coalesce to gradually convert the two phase tweed state into a single $\mathrm{LI}_{0}$ phase state. The resulting single phase microstructures are shown in the SDF images of Fig. 4.4 and Fig. 4.5 which were taken from an alloy aged for $48 \mathrm{hrs}$ and from an alloy aged for $60 \mathrm{hrs}$, respectively. From the SADP's shown in the insets, one can notice that the intensity of superlattice reflections is now markedly nonuniform among 3 variants and is indeed distributed primarily over two tariants. These two variants are denoted as $B$ and $C$ in each SADP; $A$ is mainly due to double diffraction. Consistent with this, the SDF images show attemating bright and dark regions of two variants along with the contrast due to wavy non-crystallographic Anti-Phase Boundaries (APB). The boundaries between two neighboring regions of different variants are found to be along the $<011>$ directions, which accounts for the <011> streaks in the SADP. The predominance of the two variant state was consistently observed in alloys aged under the same conditions. Yet, the relative volume fraction of two different variants was found to vary depending on a local region of a specimen.

Aging of alloys was continued at $480^{\circ} \mathrm{C}$ after aging at $450^{\circ} \mathrm{C}$ for $60 \mathrm{hrs}$. The single $\mathrm{LI}_{0}$ phase state of an alloy aged at $480^{\circ} \mathrm{C}$ for $19 \mathrm{hrs}$ is shown in Fig. 4.6. As compared with the single phase microstructures shown in Fig. 4.4. and Fig. 4.5, a few changes may be noticed. Firstly, by virtue of enhanced diffusion kinetics, Anti-Phase Domains (APD) are significantly coarsened. More prominently, the microstructure now consists overwhelmingly of one variant, suggesting that a transition from a two variant state to a mono-variant state is taking place. This may be seen, for example, by the existence of very small plate-like regions of the minor variant and by the serrated boundaries between the two variants. Although the feature 


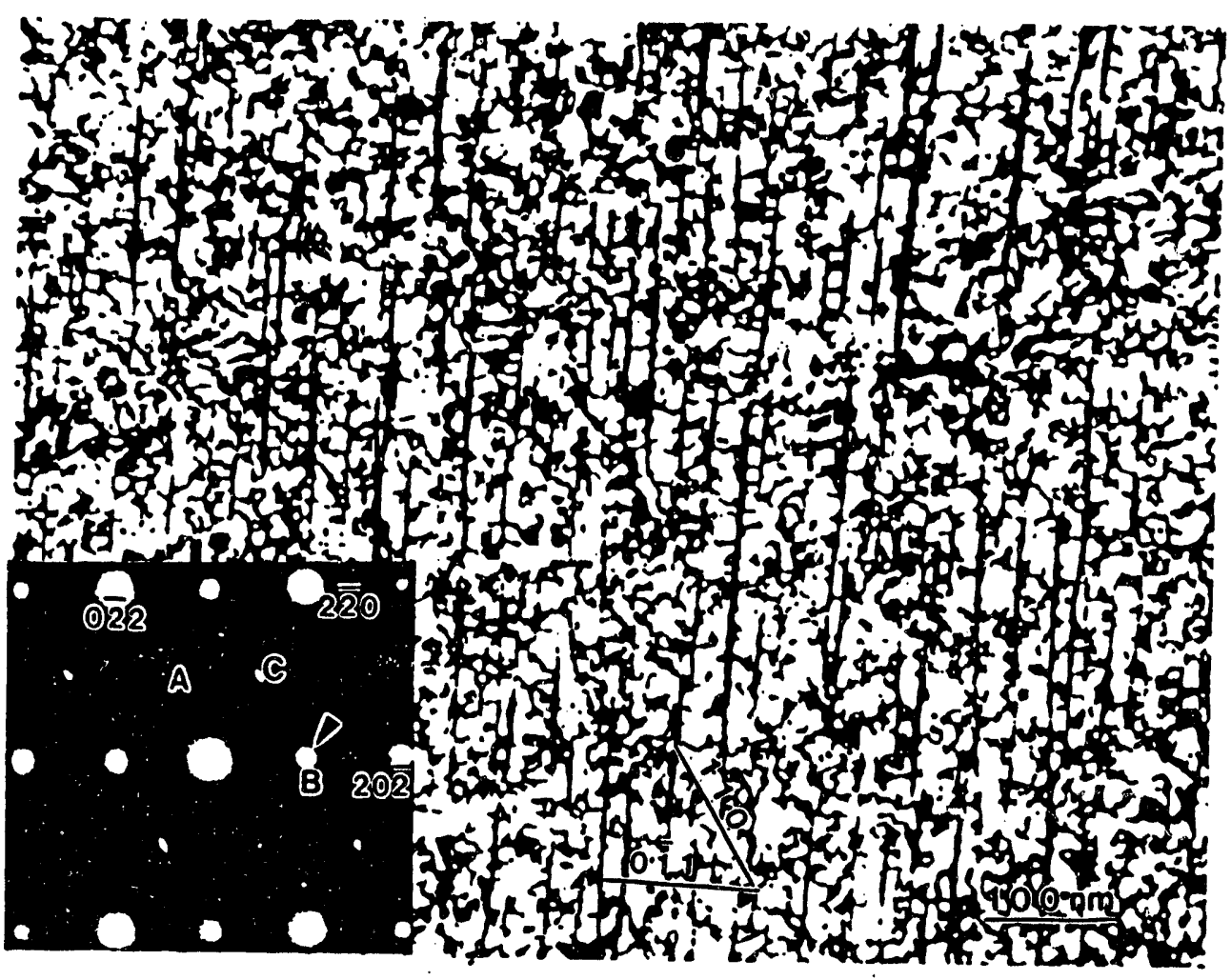

Figure 4.4: A single $L 1_{0}$ phase microstructure taken from an alloy aged at $450^{\circ} \mathrm{C}$ for $48 \mathrm{hrs:}$ it is imaged by the superlattice reflection indicated by an arrow in the SADP. The superlattice intensity denoted as $A$ is presumably due to double diffraction. 


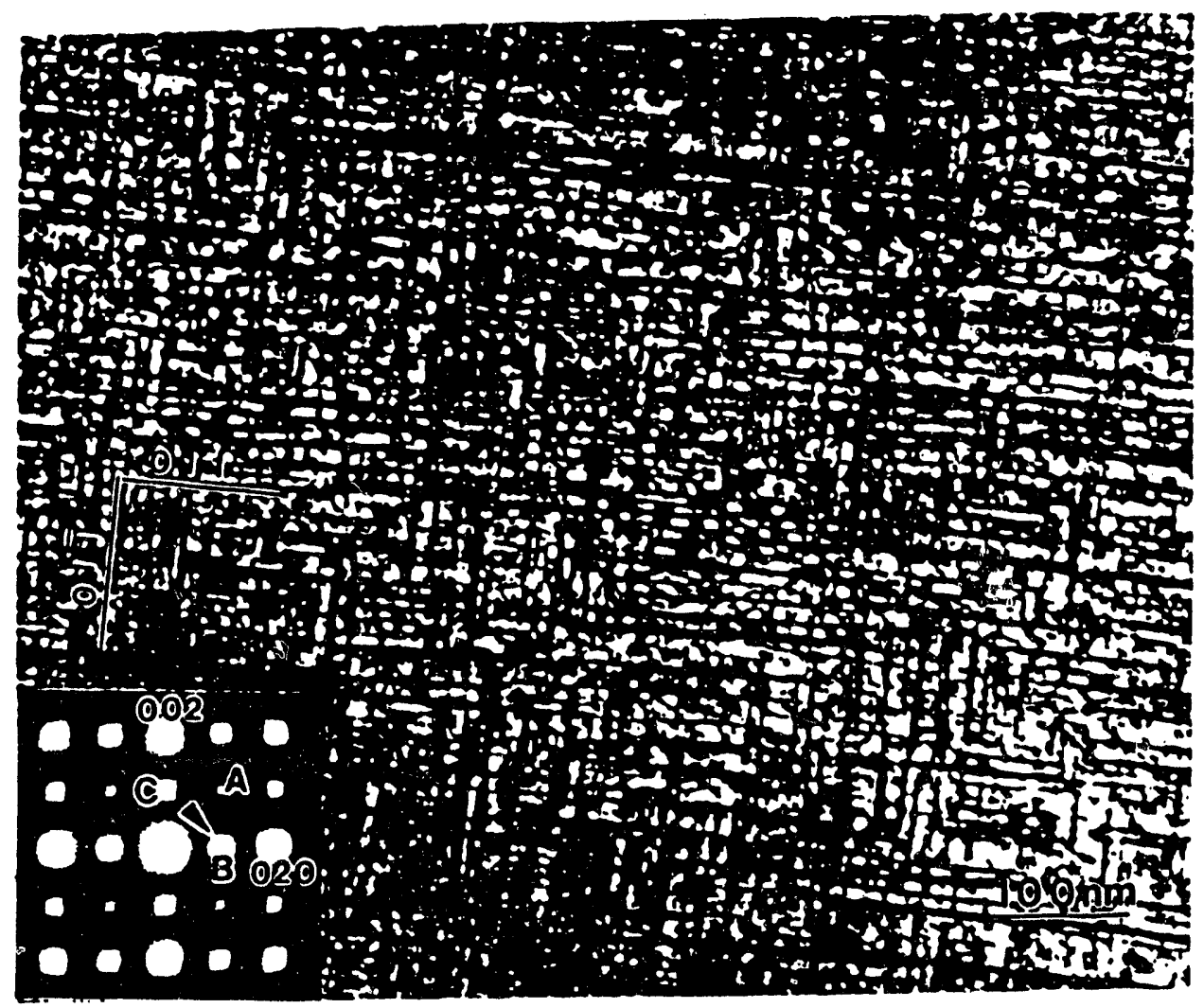

Figure 4.5: A single $\mathrm{LI}_{0}$ phase microstructure taken from an alloy aged at $450^{\circ} \mathrm{C}$ for $60 \mathrm{hrs:}$ it is imaged by the superiattice reflection indicated by an arrow in the SADP. Notice the well-developed <011> network consisting of alternating regions of two variants. The superlattice intensity denoted as $A$ is presumably due to double diffraction. 


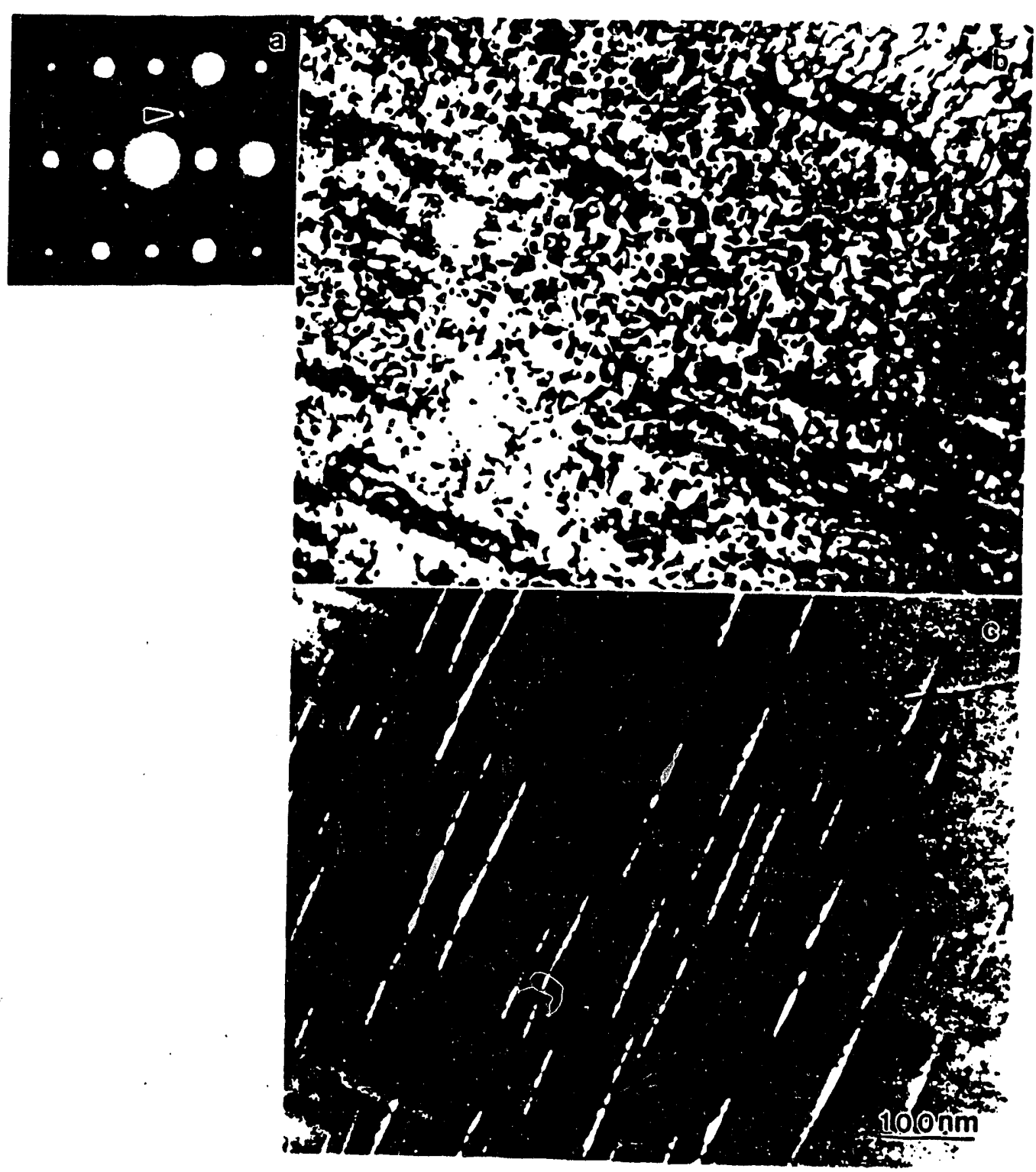

Figure 4.6: A single $L 1_{0}$ phase state of an alloy aged at $450{ }^{\circ} \mathrm{C}$ for $48 \mathrm{hrs}$ and subsequently at $480^{\circ} \mathrm{C}$ for $19 \mathrm{hrs}$. (a) [111] zone axis SADP. (b) BF image. (c) SDF image taken with the superlattice reflection indicated by an arrow. The presence of small plate-like regions of the minor variant along with the serrated boundaries between two different variants suggest that a transition from a two variant state to a monovariant state is taking place. 
presented (near monq-variant state) was rarely predominant, it was more frequently observed than it was in alloys aged at $450^{\circ} \mathrm{C}$.

A macroscopic phenomenon commonly displayed by the aged alioys was buckling of the specimen (100 $\mu \mathrm{m}$ thick). This was found to occur after a certain stage of aging and became more pronounced as aging is progressed in the single $L 1_{0}$ phase state. Since buckling is related to a non-uniform strain state and, in a single $L 1_{0}$. phase microstructure, a non-uniform strain state cap result from the region-wise variation in volume fraction of different variants, it follows that the buckling phenomenon may be ascribed to the region-wise non-uniformity in variant volume fraction. That the specimen buckles, in a sense, means that the distortion accompanying the $L 1_{0}$ ordering is able to be released through the deformation of the specimen. Convorsely, an aged specimen which is restrained from being deformed may have difficutty in relaxing the ordering-induced distórtion. Accordingly, such a specimen may be regarded as being in a relatively rigid state in the sense that we considered earlier in chapter 3. In fact, we observed that thicker specimens (200 $\mu \mathrm{m}$ thick) aged under the same condition as that of Fig. 4.6 are not in a single $\mathrm{LI}_{0}$ phase state. An unambiguous correlation, however, is not attained yot and we do not include the results in the present document.

\subsubsection{The Hablt Plane and the Shape of a Vartant Plate}

The single $L 1_{0}$ phase microstructures which we have shown above gre the prototypical ones which clearly depict plate-like regions of two different variants that are related to each other by tie $\{1110\}$ habit planes. In many cases, however, microstructures were found to display markedly different features depending on the orientations of the plates relative to the direction of observation. To our knowledge, this aspect was not examined carefully in the previous work and we believe that certain interpretations regarding the habit planes of the plates accordingly should be reevaluated. Furthermore, one can utilize the aspect to study the shape of a variant plate. Single L1 $1_{0}$ phase microstructures in two different observation conditions are shown in Fig. 4.7 and Fig. 4.8 along with the schematic diagrams illustrating 
the orientations of the variants involved with respect to the respective foil orientation (direction of observation). In the diagrams, different $\{110\}$ sections represent the $\{110\}$ plates displayed in the associated BF and SDF images.

The TEM photographs shown in Fig. 4.7 were taken along near the [110] zone axis from an alloy aged at $450^{\circ} \mathrm{C}$ for $48 \mathrm{hrs}$. A characteristic feature of the SADP is the absence of superlattice reflections. Consistent with this, no APB contrast is detectable in the BF micrograph. As Illustrated in the schematic diagram, these features may only be expected when the region under examination contains two variants with their $c$ axes making a respective angle of $45^{\circ}$ with the [110] foil oriertation. In the BF image, the regions of the variant of minor volume fraction are delineated by the loop contrast distributed throughout the matrix of the other variant. As we will discuss later in detail, this loop contrast delineates the shape of the plate on its (110) habit plane. The semi-axes of the loops are found to lie along the [110] direction (long axis) and the [001] direction (short axis).

A set of pictures taken along the [001] foil orientation of an alloy aged for $96 \mathrm{hrs}$. are presented in Fig. 4.8. A salient feature in the SADP is found to be the elongation of the superlattice reflections along the [001]" direction (in the SADP, B is due to double diffraction). In association with this, the BF and SDF images display the features which are aligned along the [010] direction (perpendicular to [001]). Both of these features are not peculiar to the 96 hr aged state but have been observed since the ordering reaction proceeded to form the network of $\{110\}$ plates. In their X-ray study on CoPt ${ }^{[88]}$ and NiPt ${ }^{89}{ }^{8}$, Tyapkin and Yamaleev interpreted the $<001\rangle^{*}$ elongation of the superlattice reflections as due to the presence of plates with $[001]$ habit planes. Pennison, Eurin and Bourret $[85,90]$ reached the same conclusion during their investigation of the $\mathrm{L}_{0}$ ordering in CoPt alloy (See Fig. $4 \mathrm{C}$ in ref. [85]). As illustrated in the schematic diagrams of Fig. 4.9, we emphasize that the ostensible [001] streaks are the 'itersections of the [101]" streaks resulting from the (101) plates with the Ewald sphere at the [100] foil orientation, not due to the plates of (001) habit plane. According to the SADP as well as to the diagrams, this conclusion may only be reached by 

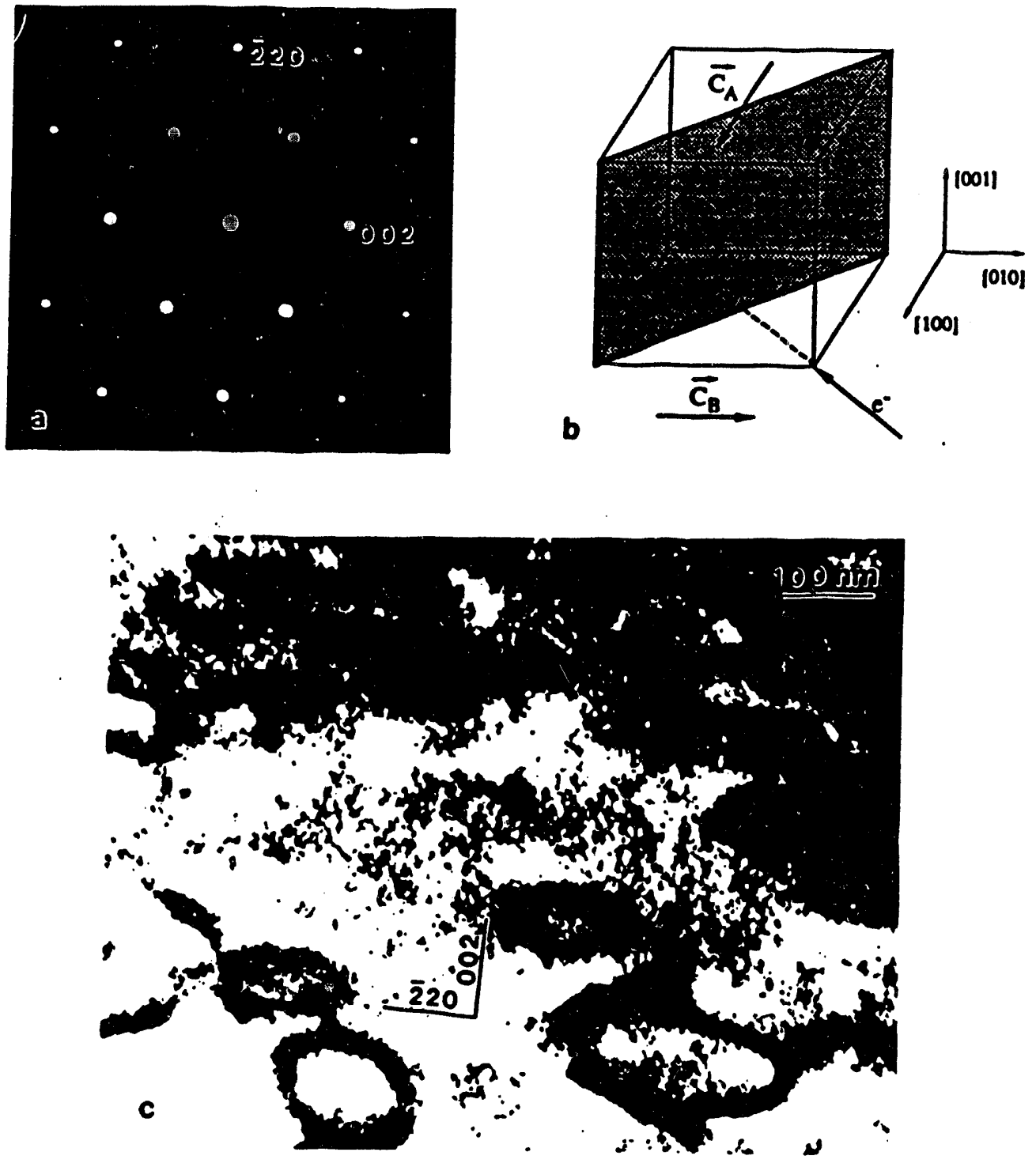

Figure 4.7: Single $L 1_{0}$ phase microstructure taken along the [110] zone axis from an alloy aged at $450^{\circ} \mathrm{C}$ for 48 hrs. (a) [110] zone axis SADP. (b) Schematic diagram illustrating the orientations of the variants involved with respect to the foil orientation. (c) BF image. From (c), notice the loop contrast which delineates the shape of a variant plate on its (110) habit plane. 

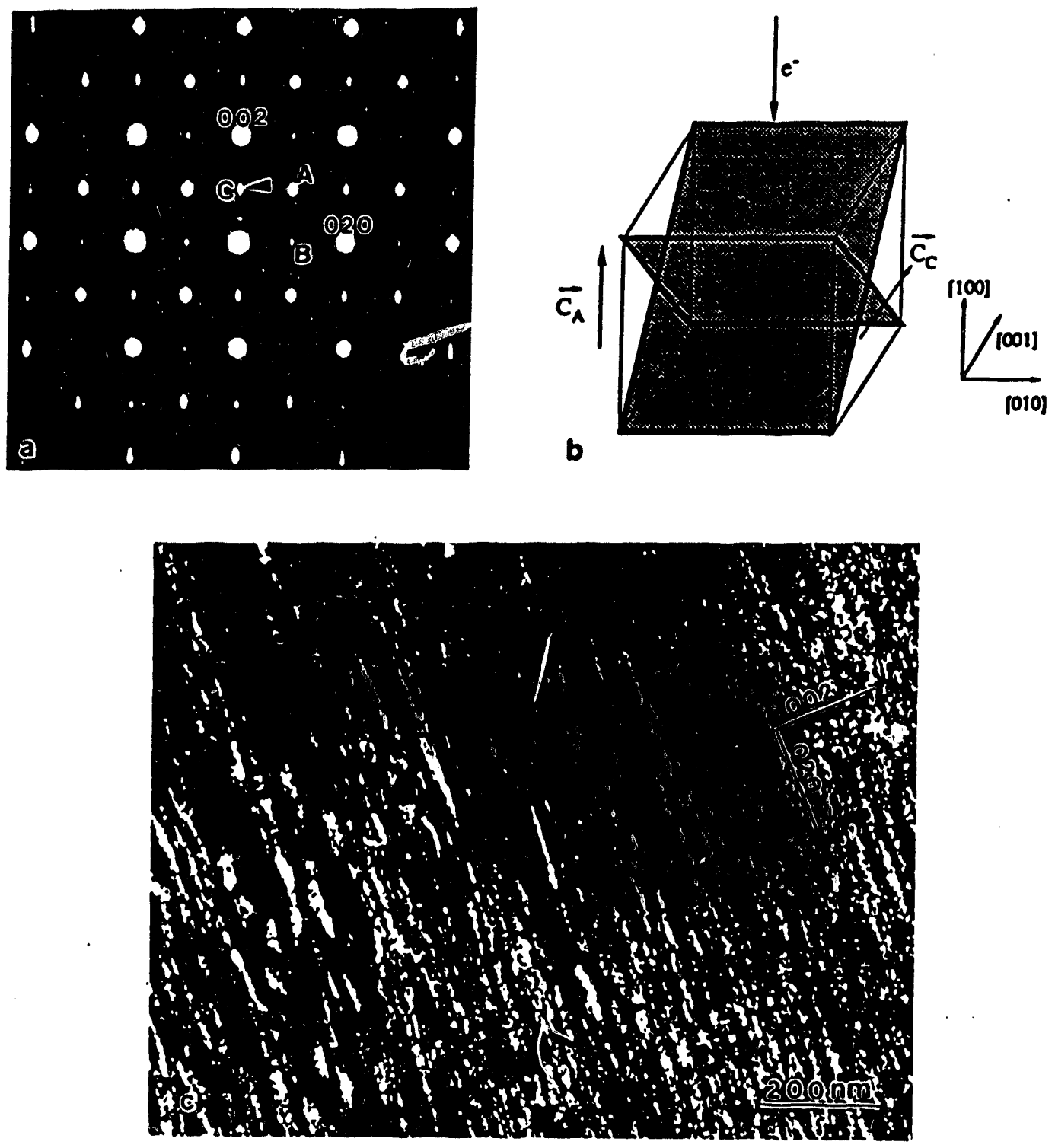

Figure 4.8: Alloy aged at $450{ }^{\circ} \mathrm{C}$ for $96 \mathrm{hrs}$, as observed along the foil normal of [100]. (a) SADP. Superfattice reflections from different variants are denoted as $A$ and $C ; B$ is presumably due to double diffraction. (b) Schematic diagram showing the orientations of the variants involved with respect to the foil normal (direction of observation). (c) SDF image taken with the superlattice reflection denoted as $C$. 


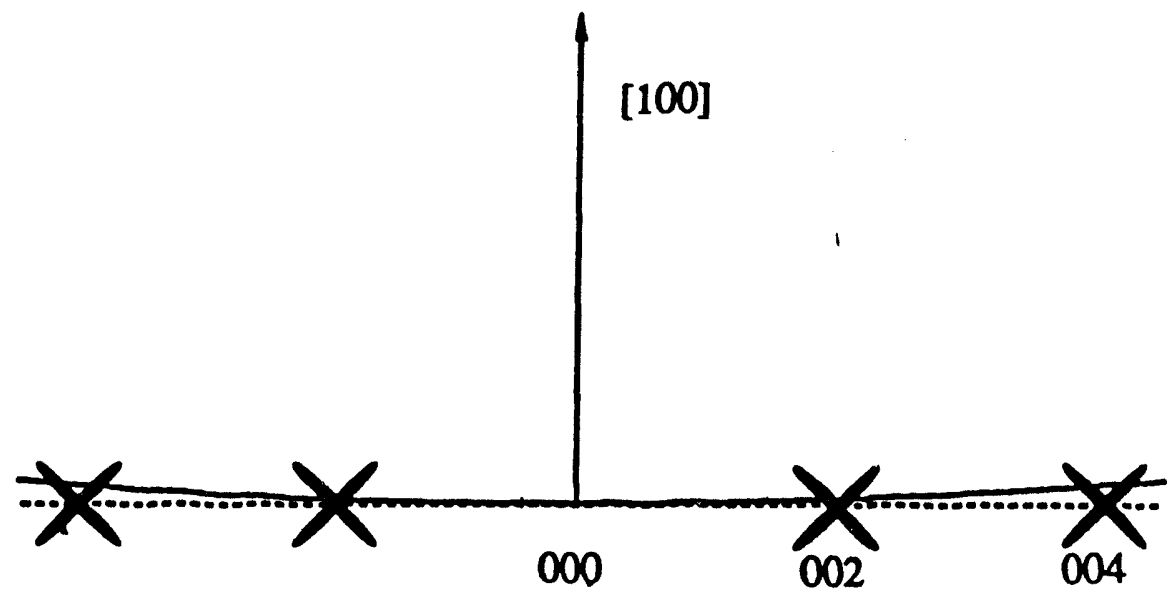

a) Side View

[100]

$\theta$

b) Top Viow

Figure 4.9: Schematic Illustrating the origin of the [001]" streaks in the SADP shown in Fig. 4.8a). These streaks are due to the intersection of $[101]^{\star}$ streaks resulting from the (101) plates with the Ewald sphere at the [100] foll orientation. 
inspection of the shape characteristics of the higher order reflections. That is, with order of reflections higher, the positions where the <101>* streaks intersect with the Ewald sphere become further away from those of the reciprocal lattice points.

\subsection{Discussion}

Ordering of a disordered fcc phase into a single $L 1_{0}$ phase is accompanied by the reduction in both translational symmetry and rotational symmetry $\left(F m \overline{3} m \rightarrow P \frac{4}{m} m m\right)$. The reduction in translational symmetry gives rise to two translational modifications and the reduction in rotational symmetry leads to three rotational modifications (or three variants): in terms of spatial regions, the former brings about two kinds of APD's and the latter three kinds of structural domains. In the context of symmetry, the entire process of fcc to $\mathrm{LI}_{0}$ ordering is, therefore, best characterized as the transformation into an aggregate of the domains of six (3x2) different modifications from an initial monodomain entity. Our TEM study shows that, in the course of this event, the spatial distribution of the domains gradually changes to certain preferred patterns from the random state produced in incipient stages of the ordering. The origin of such changes lies in the fact that each of the domains is distorted tetragonally in one of the three different orientations ( 3 variants) and the extent of the tetragonal distortion increases with the degree of long range order. In the presence of the growing distortion, essential morphological features of the single $L_{1}$ phase microstructures such as the variant habit plane, the variant volume fraction and the shape of a variant plate are determined to best accommodate the distortion.

\subsubsection{Varlant Hablt Plane}

According to the elasticity theorics of polywinin-domain monphology [20-22], for a polytwindomain complex embedded in a parent phase matrix (Fig. 4.10), the total elastic strain energy is given by the sum of two distinct elastic energy contributions:

$$
E_{\text {Tolal }}=E_{\text {Homo }}\left(\hat{\varepsilon}^{0}\right)+E_{\text {Hetero }}\left(\Delta \widehat{\varepsilon}^{\circ}\right)
$$




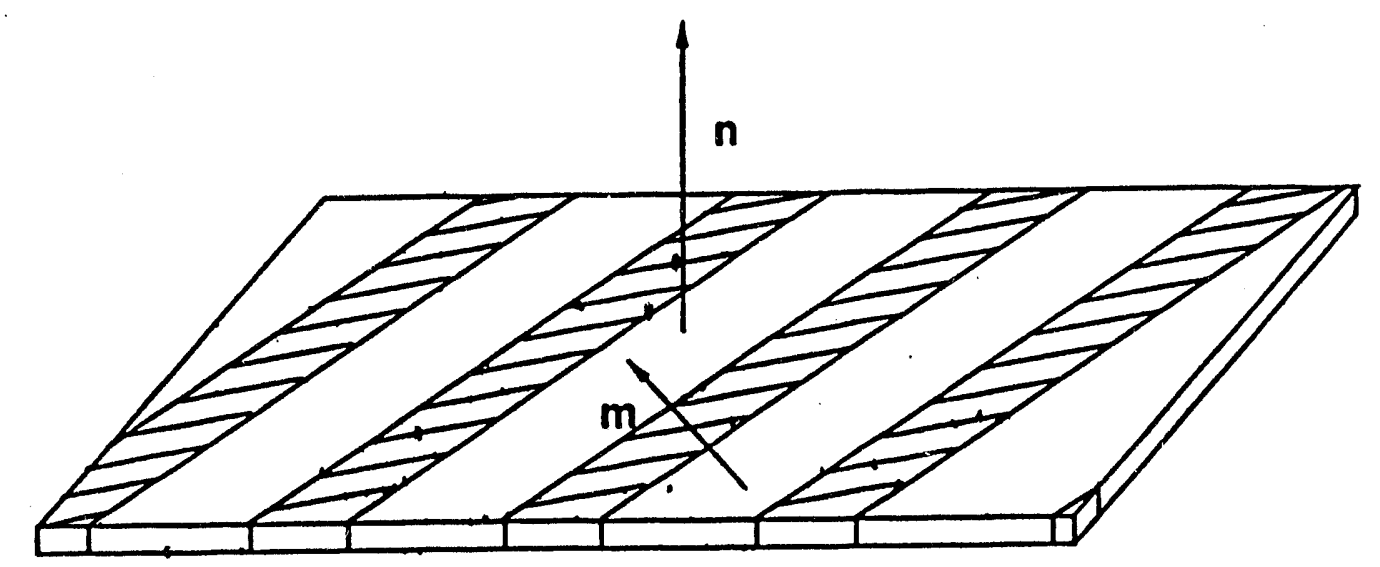

Variant 1

Variant 2

Figure 4.10: Schematic lllustrating the polytwin complex stipulated in the theories of polytwin morphology. $n$ denotes the normal of the habit plane of a complex plate with a parent phase matrix and $m$ represents the normal of the habit plane of a variant $q$ domain with a neighboring domain of the variant 2. 
where $\widehat{\varepsilon}^{\circ}$ and $\Delta \widehat{\varepsilon}^{0}$ are the total homogeneous transformation strain and the strain due to the heterogeneity of the polytwin complex, given by eq. (4.2a) and (4.2b) respectively.

$$
\begin{gathered}
\widehat{\varepsilon}^{0}=z \hat{\varepsilon}^{0}(1)+(1-z) \hat{\varepsilon}^{0}(2) \\
\Delta \hat{\varepsilon}^{0}=\widehat{\varepsilon}^{0}(1)-\hat{\varepsilon}^{0}(2)
\end{gathered}
$$

Where $z$ is the volume fraction of the variant 1 and $\hat{\varepsilon}^{0}(1)$ and $\hat{\varepsilon}^{0}(2)$ are the stress free transformation strain tensors of the variant 1 and 2 which may be represented by eq. (4.3) for a cubic to tetragonal transformation.

$$
\hat{\varepsilon}^{0}(1)=\left(\begin{array}{ccc}
\varepsilon_{33}^{\circ} & 0 & 0 \\
0 & \varepsilon_{11}^{\circ} & 0 \\
0 & 0 & \varepsilon_{11}^{0}
\end{array}\right) \quad \hat{\varepsilon}^{0}(2)=\left(\begin{array}{ccc}
\varepsilon_{11}^{0} & 0 & 0 \\
0 & \varepsilon_{33}^{\circ} & 0 \\
0 & 0 & \varepsilon_{11}^{\circ}
\end{array}\right)
$$

For the $L 1_{0}$ ordering, the strain component $\varepsilon_{i j}^{o}$ is given by $\varepsilon_{i j}^{o}=\frac{\varepsilon_{i j}^{o}(\eta=1)}{4} c^{2} \eta^{2}$ from eq. (3.30). In eq. (4.1), each elastic strain energy term consists of volume dependent and shape dependent contributions.

The theories predict that a complete relaxation of the volume dependent part of $E_{\text {Hetero }}\left(\Delta \widehat{\varepsilon}^{0}\right)$ may only be achieved when the neighboring domains of two different variants have a specific habit plane. This habit plane can be easily determined by substituting eq. (4.3) into eq. (4.2b) since the resulting strain tensor takes a planar dyadic form (eq. 2.44):

$$
\Delta \hat{\varepsilon}^{\circ} \frac{\left(\varepsilon_{33}^{\circ}-\varepsilon_{11}^{\circ}\right)}{2}(n \cdot s+s \cdot n)
$$

where $n=[110], 8=[1 \overline{1} 0]$ and vice versa. This clearly indicates that the stable variant habit plane is of the $<110>$ type. This result is always true for a cubic to tetragonal transformation regardless of the transformation mode.

\subsubsection{Varlant Volume Fraction}


An important feature which we consistently observed during the evolution of the single L1. phase microstructure was the non-uniformity in the relative population of three crystallographic variants. This was found not only to become pronounced with the progress of aging but also to vary depending on the local region of the specimen for a given aging time. A general trend was found that a two variant state is favored over a three variant state in the single $\mathrm{LI}_{0}$ phase microstructures and that a two variant state is gradually replaced by a near mono-variant state with a prolonged agng. Understanding of these aspects is thought to be important in comprehending the overall characteristics of the $L_{1}$ ordered microstructures, yet little illuminating discussion is found from the existing experimental literature. In order to advance a qualitative understanding of such aspects, we first look into the elasticity theories of polytwin-domain morphology. [20-22] The skeleton of the theories was introduced during our discussion in the previous section.

According to the theories, a complete elimination of the volume dependent part of $E_{\text {Homo }}\left(\hat{\varepsilon}^{\circ}\right.$ ) (in eq. (4.2a)) is made possible by the formation of a polytrrin domain complex in which domains of different variants are alternating with specific volume fractions. The equilibrium volume fraction $\left(z_{0}\right)$ assuring a complete relaxation of the elastic strain energy is given by eq. (4.5) when eq. (4.2a) and (4.3) are combined and the condition of invariant plane strain is applied to the resulting equation.

$$
z_{0}=\frac{\varepsilon_{33}^{o}}{\varepsilon_{33}^{o}-\varepsilon_{11}^{\circ}}
$$

It can be seen from the eq. (4.4) that unless $\varepsilon_{i 1}^{\circ}=-\varepsilon_{33}^{\circ}$, volume fractions of two different varianis in a stress free state can not be equal to each other. When the lattice parameters of the equiatomic Fe-Pd alloy [91] are used $\left(\varepsilon_{i 1}^{0}=-0.636 \varepsilon_{33}^{\circ}\right), z_{0}$ is found to be about 0.61 for the major variant.

\footnotetext{
I Eq. (4.5) is equivalent to the one due to the theory of twinned martensite [23] when the small strain approximation is taken for the latter.
} 
Although the volume fraction analysis provided by the theories renders some insight, it explains little more than a general trend, that is, the predominance of a two variant state. This is mainly because the transformation mode premised in the theories is different from that of the $\mathrm{L}_{0}$ ordering that we observed in the Fe-45at.\%Pd alloy. The analysis may be strictly valid when a transformation proceeds by a heterogeneous mode in which a system under transformation remains a heterogeneous mixture of both a parent phase and a product phase for most of the reaction time. In this case, morphology of the transformed regions of a product phase would be such that it accommodates the transformation strain under the constraint imposed by the untransformed matrix of a parent phase. This seems to explain why higher temperature ordering reactions, characterized by a rapid non-cooperative growth of a small number of nuclei, are often found to lead to the microstructures which are in good agreement with the theoretical predictions including the habit plane between a polytwin-domain complex and the matrix of a parent phase [92].

Unlike the situation treated in the theories, the $\mathrm{LI}_{0}$ ordering in the Fe-Pd alloy was seen to proceed homogeneously throughout the whole specimen region, involving a cooperative growth of a large number of nuclei i.e. homogeneous ordering. Since the parent fcc phase virtually disappears after initial stages of ordering, during the subsequent ordering process, accommodation of the transformation strain in a local region of a specimen would now be subject to the constraint due to its surroundings which are also in the process of ordering. Under these circumstances, the variant volume fraction in a local region would certainly be different from that predicted by eq. (4.4). Furthermore, it would continuously change value if the stress state of the surroundings changes with the progress of ordering. The adjustment of the variant volume fraction would remain an important means to relax $E_{\text {Hono }}\left(\varepsilon^{0}\right.$ ) (in eq. (4.1)) as long as an ordering region is substantially constrained. If the constraining ability of the surroundings diminishes, an ordering region would tend to transform to a mono-variant state which satisfies the total free energy minimum in the absence of $E_{\text {Homo }}\left(\mathcal{E}^{\circ}\right)$. This may explain why transition to a near mono-variant state is more frequently observed as buckling of specimen becomes pronounce J. The effect of a diminishing constraining ability of the 
surroundings would be particularly substantial in the regions near to free surfaces where a relatively free relaxation of the transformation strain is possible. Concerning this, an interesting result is found from the previous experimental work.

During their TEM investigation of the CuAull ordering transformation in single crystal thin films, Tong and Wayman documented the L1o (CuAul) ordered microstructures predominantly of a monovariant in a [001] oriented film [93]. In light of the line of reasoning we set out, we may point out two origins which account for the result. Firstly, due to a large undercooling at the ordering temperature employed $\left(300^{\circ} \mathrm{C}\right.$ compared to $T_{c}[\mathrm{CuAul}]=385$ ${ }^{\circ} \mathrm{C}$ ), a homogeneous ordering was made possible. In fact, they could observe only welldeveloped polytwin complexes for the CuAull ordering which can not be made to occur under a large underoooling due to the limited temperature range of phase stability $\left(T_{c}\right.$ [CuAull] $\left.=410^{\circ} \mathrm{C}\right)$. Secondly, the orientation of the thin film was such that it has enabled the variant of the favorable $c$ axis orientation to make the most of the limited constraining ability of the free surface.

In thin specimens (< $100 \mu \mathrm{m}$ ) of $\mathrm{LI}_{0}$ ordered alloys which may not have a rigid constraining ability, non-uniformity in distribution and volume fraction of different variants may lead to specimen buckling. Fig. 4.11 schematically depicts an advanced stage of the L10 ordering in a polycrystal (solid line) as well as in a single crystal (dashed line). A region enclosed by dotted lines represents a colony which is predominated by a combination of two variants out of the three possible ones. With two variants present, each colony is characterized by a homogeneous transformation strain given by eq. (4.2a). From this, it is readily expected that any two neighboring colonies may undergo the relative distortion due to the difference in the homogeneous transformation strain. For $i$ and $j$ colony, the difference in transformation strain is given by

$$
\Delta \widehat{\varepsilon}_{i j}^{0}=\widehat{\varepsilon}_{i}^{0}(z)-\widehat{\varepsilon}_{j}^{0}\left(z^{\prime}\right)
$$

where $z$ and $z^{\circ}$ are the volume fractions of major variants in each colony (notice that this equation is different from eq. (4.2b)). Similarly, one can also expect the relative distortion 


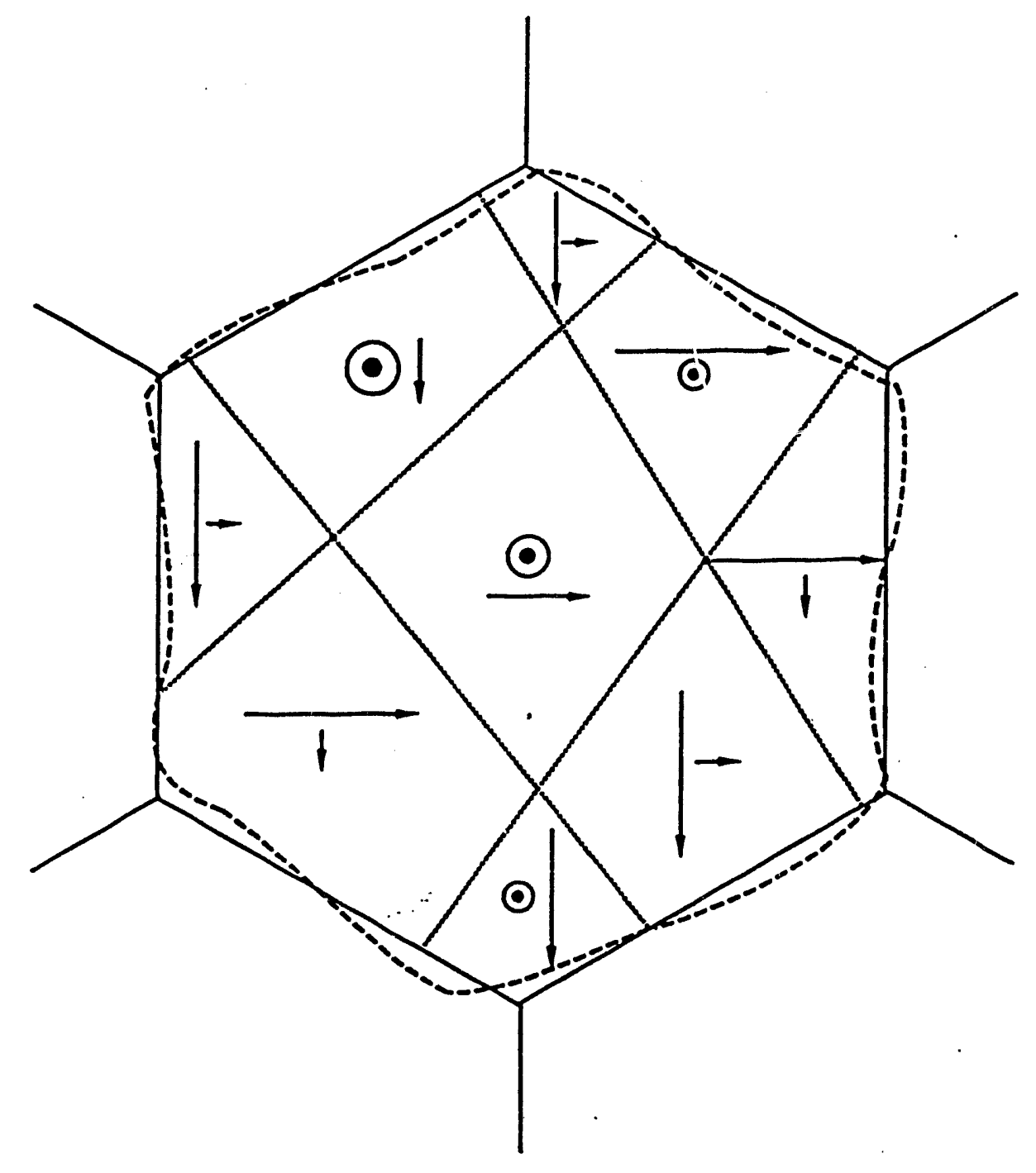

Figure 4.11: Schematic illustrating the origin of buckling of thin specimens of $L_{0}$ ordered alloys. Arrows as well as circles denote three different crystallographic variants. The non-uniformity in volume fraction of different variants is represented arbitrarily by relative magnitude of symbols in each colony enclosed by dotted lines. 
between two neighboring grains. In this case, an expression corresponding to eq. (4.5a) may be written as

$$
\Delta_{i j}=\sum_{k}^{i} f_{k} \hat{\bar{\varepsilon}}_{k}(z)-\sum_{k}^{j} f_{k} \hat{\varepsilon}_{k}^{\prime}(z)
$$

where $f_{k}$ denotes the fraction of $k$ th colony in a grain. In the second term, prime(') is used to emphasize the fact that principal axes of the homogeneous strain change from one grain to the other. The heterogeneity of strain state represented by the two equations seems to account for thin specimen buckling which becomes more pronounced with the progress of ordering.

\subsubsection{Shape of a Varlant Plate}

During our foregoing presentation of the single. $L \mathcal{I}_{0}$ phase microstructure, we have shown a characteristic feature which, to our survey, has not been reported in previous work, namely, the shape of the $\{110\}$ plate of a variant. An interesting aspect of the shape of a plate is found that it has a pronounced shape anisotropy, yet no apparent faceting. These characteristics may not be expected if the chemical interfacial energy at the plate edge is anisotropic or if it is isotropic and makes a dominant contribution to the total shapedependent energy. In such cases, the shape of a plate would be either polygonal or circular. This leads us to presume that the observed shape may be due, in a large part, to another common factor goveming the shape of a plate, that is, elastic energy contribution. In order to ascertain this reasoning and to better understand the details of the observed shape, a calculation was carried out using the elasticity theory of the shape of a coherent inclusion developed by Khachaturyan and Hairapetyan 162] (section 2.2.5).

For calculation of the shape, two variants were first selected so as to be consistent with the convention used in the BF image of Fig. 4.7: one variant with its $c$ axis parallel to [100] is taken as the matrix and the other variant with its $c$ axis parallel to [010] as an inclusion. The 
stress free transformation strain of the inclusion variant with respect to the matrix variant is given by

$$
\hat{\varepsilon}^{\circ}=\left(\begin{array}{ccc}
\frac{a}{c}-1 & 0 & 0 \\
0 & \frac{c}{a}-1 & 0 \\
0 & 0 & 0
\end{array}\right)
$$

where $a$ and $c$ are the lattice parameters of the $L 1_{0}$ phase. From an electron diffraction pattern of the $48 \mathrm{hr}$ aged state (corresponding to the state of Fig. 4.7), the cla ratio was measured to be about 0.96 . With the resulting transformation strain (eq. (4.6)), the variant habit plane is found to be $[1.02,1,0]$ which is slightly away from [110] (notice the difference between eq. (4.4) and eq. (4.6) in terms of the reference state with respect to which strain is measured). By use of these values in eq. (2.39), parametric equations (2.55a) and (2.55b) were solved numerically. Since the elastic constants of the $\mathrm{Fe}-45$ at.\% Pd alloy are not known, calculations were made for two possible bounding ratios of $C_{12} / C_{11}$ based on the room temperature elastic constants data of the Fe-32.5 at.\% Pd disordered alloy recently measured by Muto et al. [19]: $C_{11} \sim 16 \times 10^{10} \mathrm{~N} / \mathrm{m}^{2}, C_{12} \sim 13.8 \times 10^{10} \mathrm{~N} / \mathrm{m}^{2}$ and $C_{44} \sim 7.3 \times$ $10^{10} \mathrm{~N} / \mathrm{m}^{2}$. The results are shown in Fig. 4.12. One can notice that the calculated shapes are in good agreement with the observed shape (Fig. 4.7c) in two important aspects. Firstly, the calculated shapes correctly reproduce the directions of the long and the short axes of the loop-contrast. Since shape-dependent elastic energy is effectively that of a dislocation loop (see eq. (2.53)), we may take [001] as the direction of a low line tension and [110] as that of a high line tension. In addition to this, the aspect ratios are also found to be fairly close to the experimental values $(0.38 \sim 0.5)$.

\subsection{Conclusion}

By use of the Fe-45 at.\%Pd alloy, we have studied the effect of tetragonal lattice distortion on the morphology of the $\mathrm{LI}_{0}$ ordering microstructure with a particular emphasis on the single $L 1_{0}$ phase state. It was shown that essential morphological features such as the 

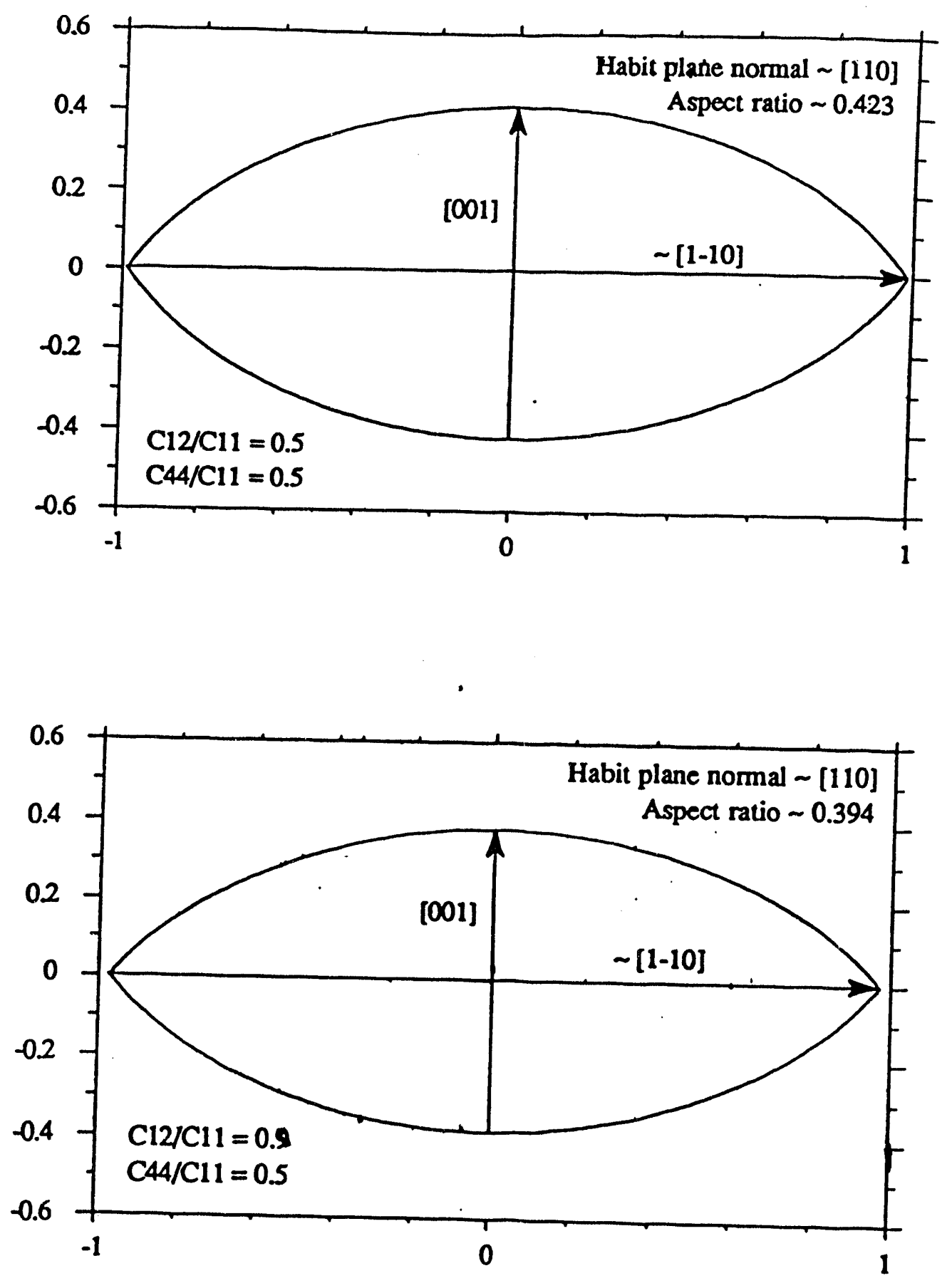

Figure 4.12: Calculated shapes of a plate-like region of the variant 1 (with $c$ axis parallel to the [100] direction) coherently embedded in a region of the variant 2 (with $c$ axis parallel to the [010] direction). Compare these with the observed shape shown in Fig. 4.7c). 
variant habit plane, the variant volume fraction and the shape of a variant plate are determined so as to best accommodate the tetragonal distortion accompanying the $\mathrm{L}_{0}$ ordering. For the variant habit planes, we have shown unequivocally that $\{110\}$ planes are the unique optimum habit planes, in agreement with the theoretical prediction as well as with the bulk of existing experimental observations. As for the variant volume fraction, a morphological aspect of utmost importance in regard to the relaxation of the homogeneous transformation strain, a characteristic sequential change was observed with the progress of ordering. Initially from the three variant state, the variant volume fraction in a local region was found to vary gradually through the two variant state until a near mono-variant state is reached. Insightful as they are, theories of polytwin morphology shed little light on the nature of this sequential change. We have explained this in terms of the dynamic interplay between the variant volume fraction and the diminishing constraining ability of the ordering surroundings. This interplay was suggested to be particularly substantial when the geometry of the specimen does not provide a rigid constraining ability. Along the same line of reasoning, buckling phenomenon was explained. In case of a two variant state, the shape of a variant plate embedded in the matrix of the other variant was found to be bop-like with an aspect ratio of $0.38 \sim 0.5$. By use of the elasticity theory of the shape of a plate-like precipitate, a calculation was made to result in a good agreement with the experimental result. 


\section{Bain Strain Relaxation during Decomposition of Hyper- eutectoid Cu-Be alloys.}

In this chapter, we investigate another important class of cubic to tetragonal crystal lattice reconstruction which often occurrs in conjunction with diffusional atomic redistribution processes such as the Bain structural transformation. By careful documentation of the decomposition processes of hyper-eutectoid $(\beta: b c c)$ CuBe alloys, we will establish a better understanding as to how a radical change in crystal structure (bcc to $f c c$ ) is achieved, when accompanied by atomic ordering and compositional phase separation. In doing this, we adopt the line of reasoning advanced by Khachaturyan and Laughlin [34] in their recent theoretical treatment of decomposition of $\alpha(f c c)$ CuBe alloys. It tums out that elasticity is a vital conceptual framework whereon the major features of decomposition may be put into a right perspective.

Throughout this study, we place our primary focus on the early stages of decomposition process, typically represented by as-quenched states. Due to the remarkably rapid decomposition kinetics of CuBe alloys, many characteristic events occur during the early stages. Therefore, a precise understanding of as-quenched states is very important to comprehend the overall picture. In section 5.2.1, we characterize the principal mechanisms of the Bain strain relaxation during early stages of decomposition by examining two different as-quenched states with conventional TEM and high resolution TEM (HRTEM). In section 5.2.2, we then look in'o later stages of Bain strain relaxation with alloys of two different thermal histories (air-cooled and quenched/aged) and examine what differences in precipitate morphology may be brought about by changes in kinetic paths of decomposition. 
This chapter closes with discussion of experimental results in section 5.3.

\subsection{Experimental Procedure}

Cu-Be binary alloys containing 9.04 wt.\%Be (41.2 at.\% Be) were furnished by BrushWellman Inc. in the as-cast condition. The as-cast alloys were homogenized for one day at $840^{\circ} \mathrm{C}$ and were sliced into plates about $0.5 \mathrm{~mm}$ thick. Subsequently, the specimens were cold-rolled to thicknesses of about $0.2 \mathrm{~mm}$. Due to the brittleness of specimens, further reduction in thickness was made by mechanical polishing. Discs of $3 \mathrm{~mm}$ in dia. were punched out from these specimens and were then given a disordering treatment for $1 \mathrm{hr}$. at $840^{\circ} \mathrm{C}$. For each of these heat treatments, the specimens were encapsulated in argon backfilled quartz tubes. The disordered specimens were quenched into iced-brine water. Quenching of the disordered specimens was performed by rapidly transferring a tube and smashing it onto a heavy metal object which was immersed in the quenchant.

Specimens for transmission electron microscopy (TEM) were prepared using a twin-jet electro-polisher with an electrolyte of 70 vol. \% methanol and 30 vol. \% nitric acid below -30 ${ }^{\circ} \mathrm{C}$ and at $10 \mathrm{~V}$. When necessary, electro-polished samples were ion-milled for short periods of time (< $10 \mathrm{~min}$. ) at 4-5 keV and $0.2 \mathrm{~mA}$. Conventional TEM was performed using Philips $420 \mathrm{~T}$ operating at $120 \mathrm{kV}$. High Resolution TEM was conducted with JEOL $4000 \mathrm{EX}$ at 400 kV.

\subsection{Experimental Results}

\subsubsection{Baln Strain Relaxation In As-quenched States.}

\subsubsection{Overall Characteristics of a Rapldly As-quenched State.}

The kinetics of decomposition of the $\beta(b c c)$ phase are remarkably fast and the early stages of the decomposition process could not be suppressed by rapid quenching. The 
overall nature of a typical as-quenched state is shown by a series of photographs in Fig. 5.1 taken near the [100] zone axis of the ordered $\gamma(\mathrm{B2})$ matrix phase. From the micrographs, the precipitates are found to be ven thin $(6 \sim 20$ A) plates with their habit plane normals nearly parallel to the $[010]_{\gamma}$ and $[001]_{\gamma}$ directions of the ordered $\gamma$ matrix. Corresponding to this, the precipitate reflections (e.g. spot 1) are elongated along the same directions in the SADP (Selected Area Diffraction Pattern). The streaks around the matrix $\gamma$ reflections (e.g. spot 2) are presumed to result from double diffraction as well as from thin distorted layers of the matrix adjacent to the broad faces of the precipitate plates.

On close inspection of the BF (Bright Field) image (Fig. 5.1b) and of the DF (Dark Field) image (Fig. 5.1d) taken with a precipitate reflection (spot 1), one can find a characteristic striation contrast inside the precipitate plates. By contrast, in the SDF (Superlattice Dark Fieid) image (Fig. 5.1C) taken with a matrix reflection (spot 2), the plates are imaged as dark regions with no striation contrast inside. This suggests that the contrast can not be due to thin $\gamma$ phase regions, as proposed in an early TEM investigation [38] but may be due to certain types of planar defects existing in the precipitate plates. The nature of the defects, however, is not immediately clear from the photographs shown. It is interesting to note, from the SDF image, that no APB (Anti Phase Boundany) contrast is detectable in the ordered $\gamma$ matrix. Considering that extensive APB coarsening may not have been possible during quenching, we are led to presume that the APB's may have been consumed by the Cu-rich precipitates. In conjunction with the phase diagram features of hyper-euctectoid alloys (see Fig. 1.3b), this suggests that earliest stages of the decomposition process commence with bcc to B2 ordering, which is then followed by precipitation of coherent $\mathrm{Cu}$-rich $\{001\}_{Y}$ thin plates at the APB's of the B2 matrix.

The $[100]_{\gamma} z o n e$ axis pattern shown in Fig 5.2a) provides more information on the structural aspects of the precipitate phase. The SADP was taken from a region including that of the BF image shown in Fig. 5.2c). We first carry out a cursory analysis of the pattern, disregarding subtle diffraction effects such as streak-like intensity distribution around the 

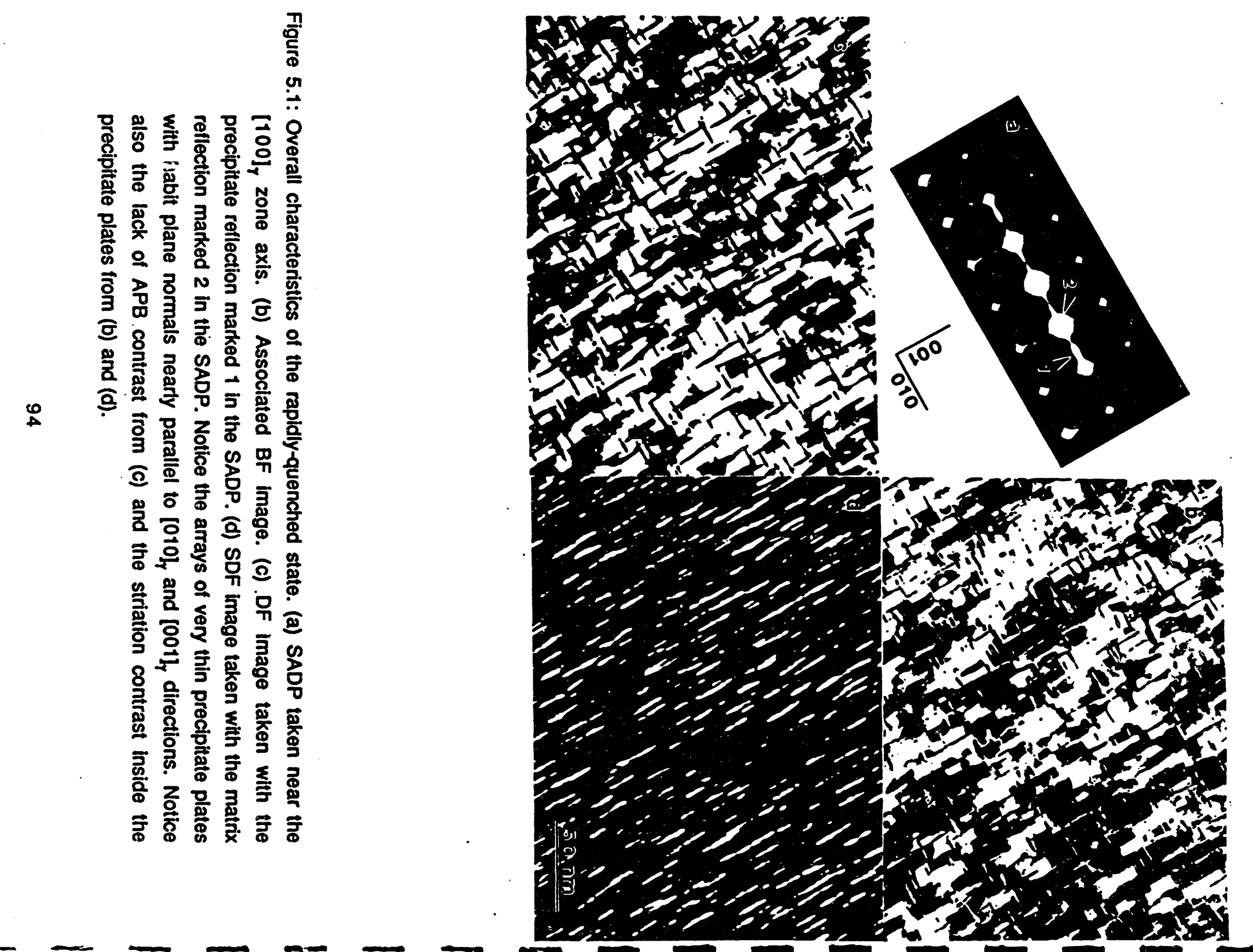

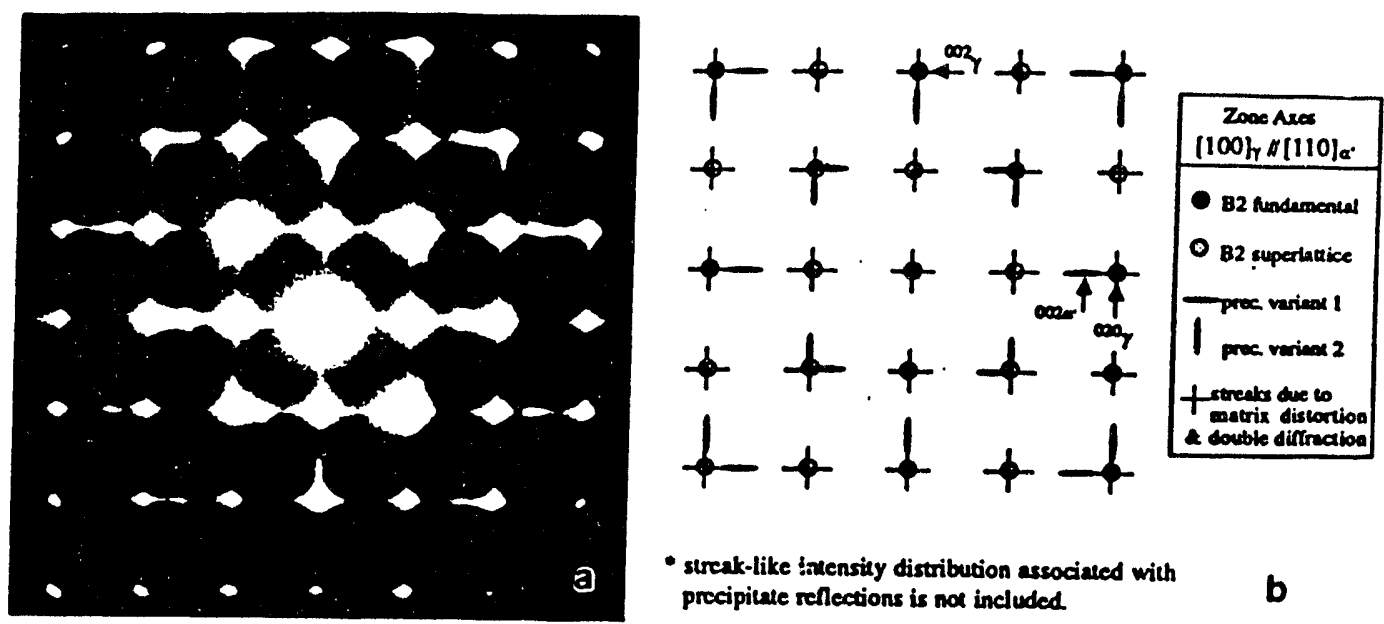

- streak-like fntensiky distribution associated with precipilate reflections is not included.

b

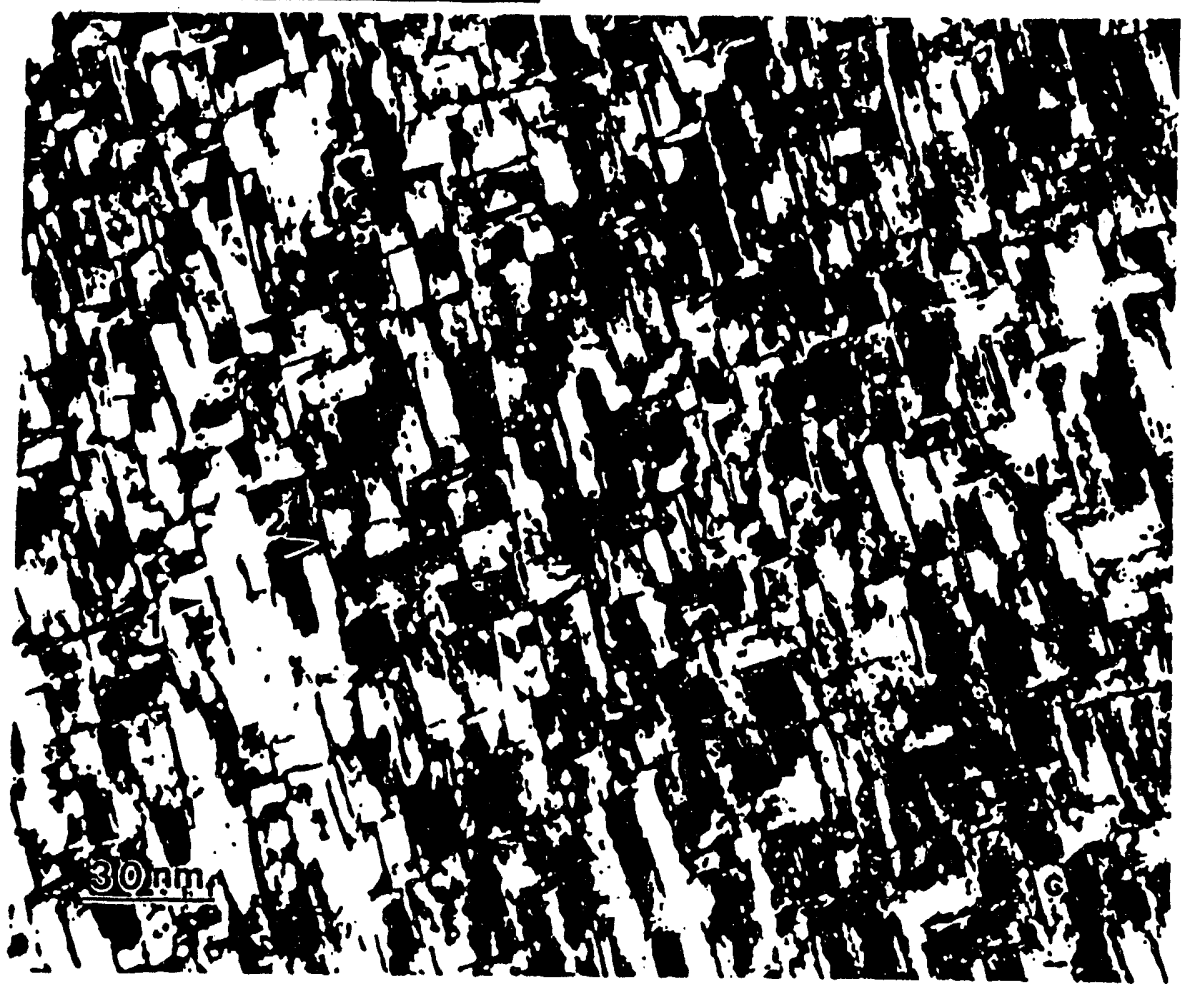

Figure 5.2: (a) [100] zone axis SADP. (b) Schematic illustration of the basic pattem of the SADP $\left([100]_{\gamma} / /[100]_{\alpha}\right)$. (c) BF image associated with (a). Two different kinds of the striation contrast are marked 1 and 2 in the BF image. 
precipitate reflections. In Fig. 5.2b), the zone axis patterns of two precipitate variants are schematically reproduced, being superimposed on the $[100]_{\gamma} z 0$ ne axis pattem of the matrix $\gamma$ phase. From the array of precipitate reflections due to one variant, it is found that the constrained crystal structure of the precipitate phase is fot (we will designate fct as $\alpha^{\prime}$ ): a more detailed analysis shows the phase to be distorted from fat with a slight monoclinicity. Based on Fig. 5.2a), the approximate orientation relationship between the matrix and the precipiate is determined to be

$$
\begin{array}{lll}
{[100]_{\gamma} / /[110]_{\alpha^{\prime}}} & \text { or } & {[\overline{110}]_{\gamma} /\left[(010]_{\alpha^{\prime}}\right.} \\
(001)_{\gamma} / /(001)_{\alpha^{\prime}} & & (001)_{\gamma} / /(001)_{\alpha^{\circ}}
\end{array}
$$

which is the Bain lattice correspondence; the second orientation relationship was attained from the $[110]_{\gamma} z o n e$ axis pattem. An estimation of the Bain strain relaxation may be then made in terms of the tetragonality $(t)$ of the fot structure. If coherency on the broad face of a plate is assumed, i.e. $a_{\alpha}=\sqrt{2} a_{p}$, a tetragonality of $i=g_{002}^{\gamma} l / \sqrt{2} \lg _{002}^{\alpha} l=0.84$ is calculated where $a$ and $\mathbf{g}$ denote the lattice parameter and the diffraction vector in the SADP, respectively. Since the equilibrium tetragorality value would be $t_{\text {amil }}=0.9425$, this indicates that about half of the Bain transformation strain is already. accommodated in the as-quenched state presented.

Characterization of the $\alpha^{\prime}$ precipitate was first made by Tadaki, Sahara and Shimizu [38] and Auvray [39] in independent TEM investigations. In each work, the precipitate was proposed to be respectively a metastable phase or an intermediate phase, distinguished from the stable equiltbrium $\alpha(f c c)$ phase. As we will discuss in detail later in section 5.3, we emphasize that $\alpha^{\cdot}$ precipitate is an elastically unstable transient state. This unstable nature of the precipitate leads to a rapid development of the processes which prompt a further relaxation of the Bain strain. In this respect, it is interesting to note from the BF image that the habit planes of many precipitate plates are slightly deviated from the. $<001\rangle_{\gamma}$ directions either globally over a whole plate or segmentwise. In addition, striation contrast is quite noticeable inside the precipitate plates (notice also the associated streak-like intensity in the 
SADP.). On close inspection, we find that the striation contrast may be differentiated into two kinds and they are marked 1 and 2 in the BF image (these will be named type 1 and type 2 contrast, respectively). Considering the fact that these are possible planar defects which may further the relaxation of the Bain strain, one is faced with two possibilities i.e. they are either stacking faults or twins ${ }^{\dagger}$. The habit plane rotation and the formation of stacking faults and twins represent the elementary mechanisms of Bain strain relaxation during the ensuing stages of decomposition.

With the additional relaxation induced by the features discussed, the precipitates would no longer stay in the elastically constrained state caused by the coherency across the $\{001\}_{\gamma}$ habit. Naturally, the crystal lattice of the precipitates is distorted from the $\alpha^{\prime}(f c t)$ state depending on the extent and the nature of the additional relaxation. This may involve not only a change in the lattice constants but also rotation of the precipitate crystal lattice with respect to that of the matrix. This is because the distorted crystals would find themselves incompatible with the matrix if they maintained the orientation which was perfect for the $\alpha^{\prime}$ state. When monitoring such cystal deformation in reciprocal space, one should be able to see the corresponding changes in direction as well as in magnitude of the $g$ vectors. Indeed, one can notice from the SADP (Fig. 5.2a) that the precipitate reflections are not sharply elongated but have the shape of a tadpole, in spite of the very thin nature of the precipitate plates. We may best understand the shape by envisaging the head part of a tadpole shape as due to a range of precipitate crystal lattices which are slightly different from each other in the qxtent of Bain strain relaxation.

On the basis of the perspective advanced above, we now start to examine in more detail the planar defects in the as-quenched alloys. We will look into habit plane rotation later when we discuss the aged states and the air-cooled states.

\footnotetext{
t Unlike stacking faults, twins are obviously not planar dofects bet generate them in the form of win boundaries. For convenience, however, we will use the term "planar delect when we need to refer to both collectively.
} 


\subsubsection{Polydomaln Preclpltate Plates in As-Quenched States.}

The micrographs shown in Fig. 5.3a) and 5.3b) were taken from another quenched specimen. Although the quenching rate was not intentionally varied, the microstructure is rather coarse unlike the ones previously shown. For convenience, we will denote this relatively slower-quenched alloy as Q2 and the rapidly quenched alloys as Q1. The coarse microstructure of the Q2 alloy again suggests that the kinetics of the early stages of decomposition is very fast and even a slight difference in the quenching rate can give rise to a significant difference in the as-quenched microstructure. In the BF image, precipitate plates with the type 1 striation contrast are more frequently observed than those with the type 2 contrast and the ones with no contrast. Distinct from the others, plates with the type 1 contrast display a saw-tooth morphology. This morphology is featured by a slight rotation of two broad segments neighboring a thin band (10 - 30 A thick) from the $<001\rangle_{\gamma}$ direction. In association with this, the SADP shows some precipitate reflections which are slightly displaced from the reflection positions due to the plates constrained by $\{001\}_{\gamma}$ habits.

Fig. 5.3b) shows the DF Image taken with the streaked precipitate reflection $\left(g_{1 i}\right)$ Indicated in the SADP. The most prominent features are that in each single plate, both thin and broad regions are in opposite contrast and that for each type of region, the contrast reverses between two orthogonal sets of the plates with $\sim\{001\}_{\gamma}$ habits. This indicates that the crystal lattice of the thin regions is in a very different spatial orientation from that of the broad regions in the same plate and that the thin regions in one set of plates have a similar crystal lattice orientation as the broad regions in plates of the other set. This is clearly expected of the plates containing polytwin domains. Other important features to note are as follows. Firstly, the proportion of the broad and thin regions in a plate is systematic. It is measured to be approximately $4: 1$. Secondly, all of the thin regions of bright contrast are positioned in the same direction. By rotation calibration, it is found that the plane nomal of these thin regions coincides with the direction of the streak-like intensity in the SADP i.e. $g_{1 \overline{1}}^{\text {. }}$ 


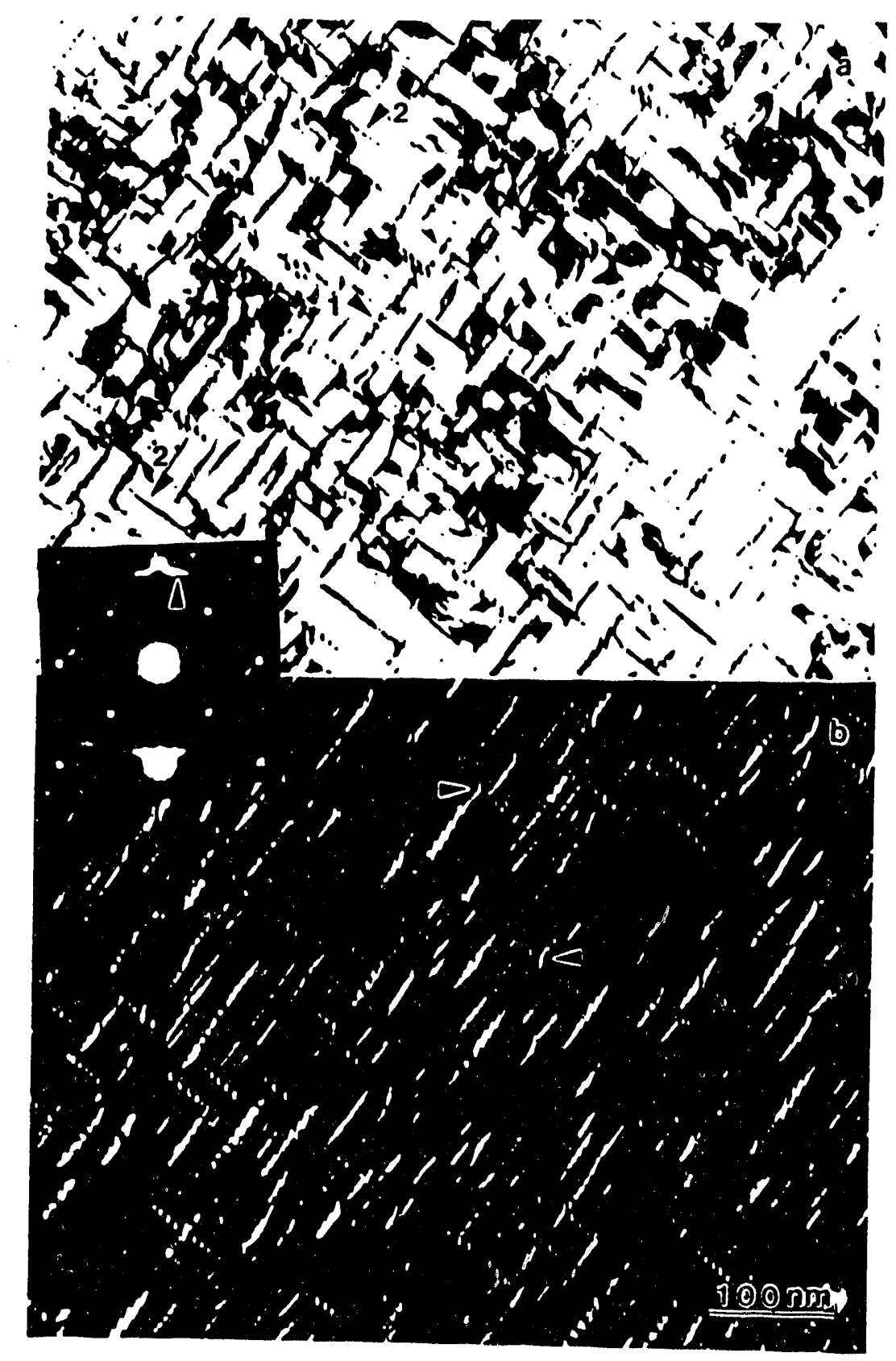

Figure 5.3: Microstructure of the Q2 alloy. (a) BF image. (b) DF image taken with the precipitate reflection $\left(g_{11}^{\text {Paj }}\right)$ indicated in the associated SADP. As compared to the case of Q1 alloys (Figure 5.1 and Figure 5.2), precipitate plates are noticeably coarser. Majority of precipitate plates display a characteristic saw-tooth morphology which is expected of polytwin plates. 
A [100] zone axis pattern taken from the same region of the specimen, is presented in Fig. 5.4a). Two characteristic features are readily noticable and they are marked by arrows: twofold-split distinct precipitate reflections and the streak-like intensity near those reflections. The origins of these features are schematically illustrated in Fig. 5.4C). In the diagrams, numeric symbols represent orientational variants with each one characterizing a different orientation of the precipltate crystal lattice. For example, $3 \cdot 2^{\mathrm{T}}$ denotes the twin of the second minor orientational variant of the major variant 3: major variants 1, 2 and 3 refer to the precipltate plates whose habit normals were initially parallel to $[100]_{\gamma}[010]_{\gamma}$ and $[001]_{\gamma}$ respectively. The symbols $3 \cdot 1(T)$ and $3 \cdot 2(T)$ denote the twin planes associated with the variants 3.1 and 3.2, respectively. From the schematic diagrams, one can clearly see that broad twin domains are responsible for the twofold-splitting of distinct precipitate reflections and that thin twin domains account for the streak-like intensity. By extending the procedure depicted in Fig. 5.4C) to other low order reflections, we constructed the diffraction pattem shown schematically in Fig. 5.4b). The resulting pattem successfully reproduces most of the salient aspects of the actual SADP. The intensity maxima along four outer fractional arcs in Fig. 5.4a) can also be reproduced by translation of the low order precipitate reflections in Fig. 5.4b). A good agreement between the two patterns appears to lend an indirect support to the assumption that we used for the construction. That is, $[110]_{\text {prec }} / /[100]_{\gamma}$ remains an invariant axis during the rotation of the segment habit and hence of the precipitate crystal lattice as the precipitates relax from the $\alpha^{\cdot}$ state by the formation of polytwin domains. With the aid of the schematic SADP (Fig. 5.4b), we may now clearty understand the contrast in DF image of Fig 5.3b). That is, DF imaging of the precipltate reflections within the circled area of Fig. 5.4b) should produce bright contrast for the domains due to 2.1 and $3.2^{\mathrm{T}}$ ( 2.1 denotes major domains in the plates rotated $90^{\circ}$ clockwise with respect to the plates containing 3.1)

The interpretation that we advanced regarding the streak-like intensity due to the thin twin regions enables us to distinguish it from the streak-like intensity due to the other type of planar defects, that is, stacking faults. As shown in Fig. 5.5 by enlarged views, the streak-like intensity in Fig. 5.2a) (differs in both positions and directions from the one due to thin twin 

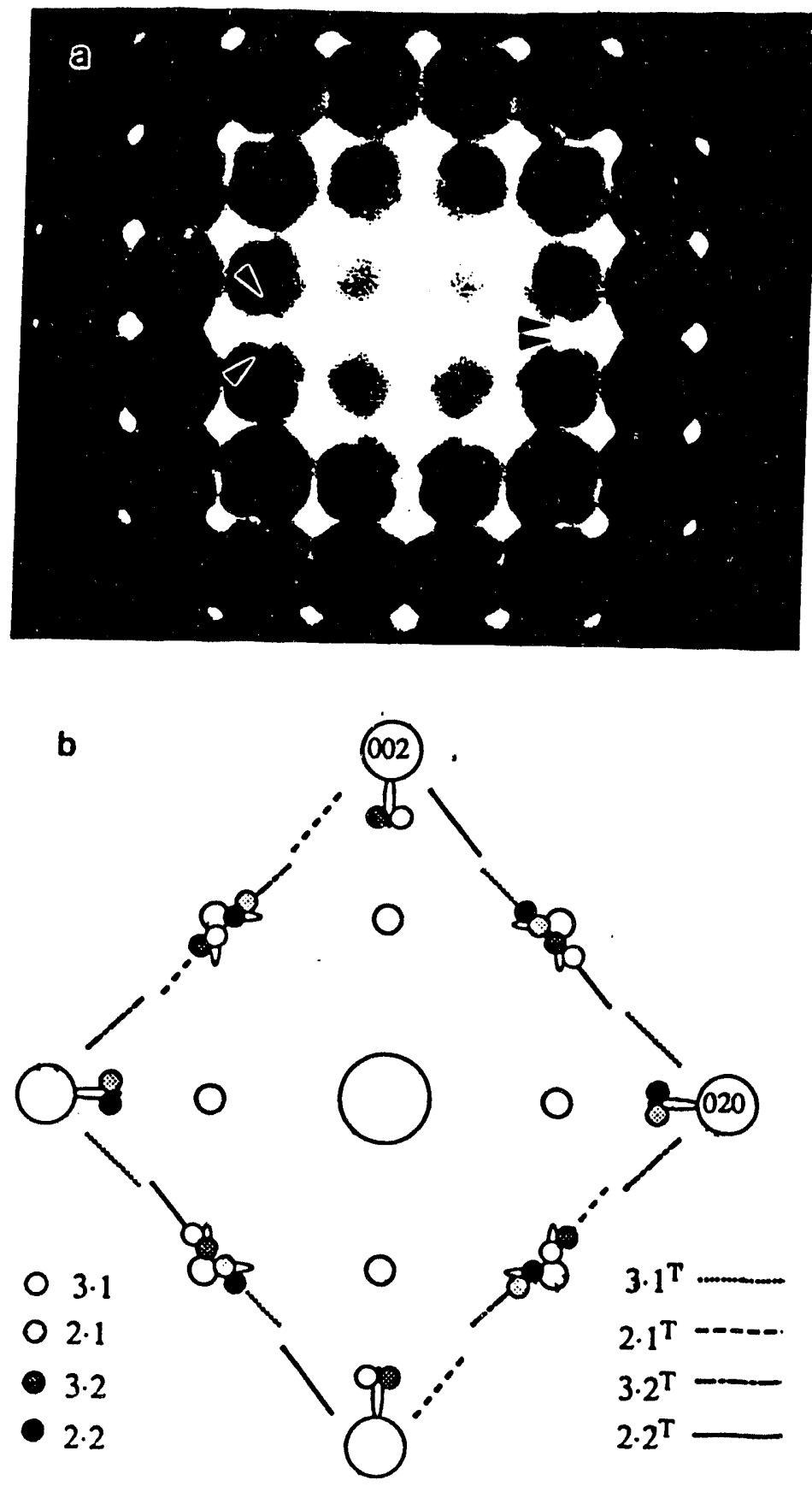

Figure 5.4: (a) SADP taken from the same region of the 22 alloy shown in Fig. 5.3 along the $[100]_{y}$ zone axis.. (b) Schematic Illustrating the constitution of the SADP. 

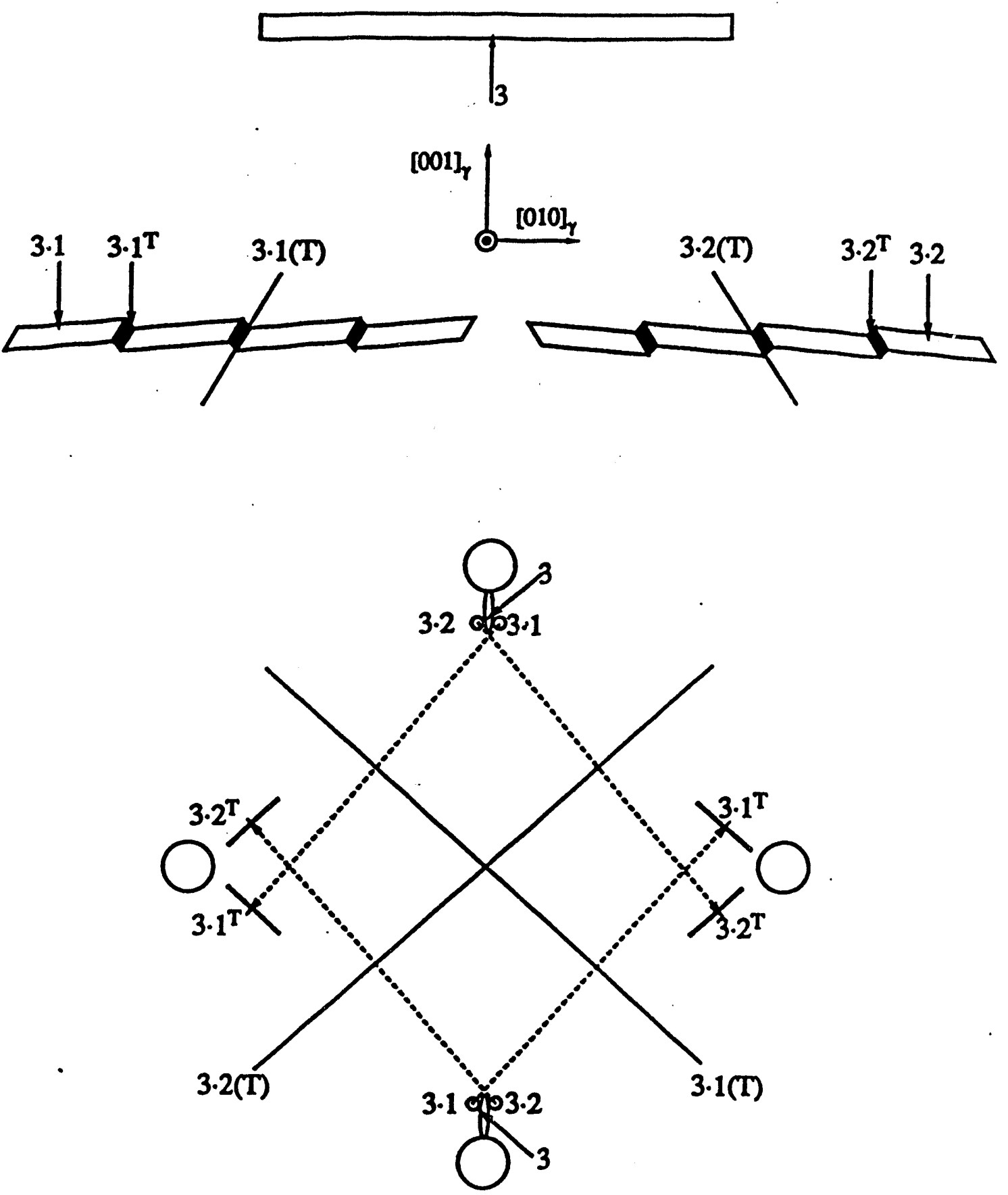

Figure 5.4: cont. (c) Schematic illustrating the origins of the two fold-split distinct precipitate reflections and of the streak-like intensity. Each of these features is marked by arrows in (a). 

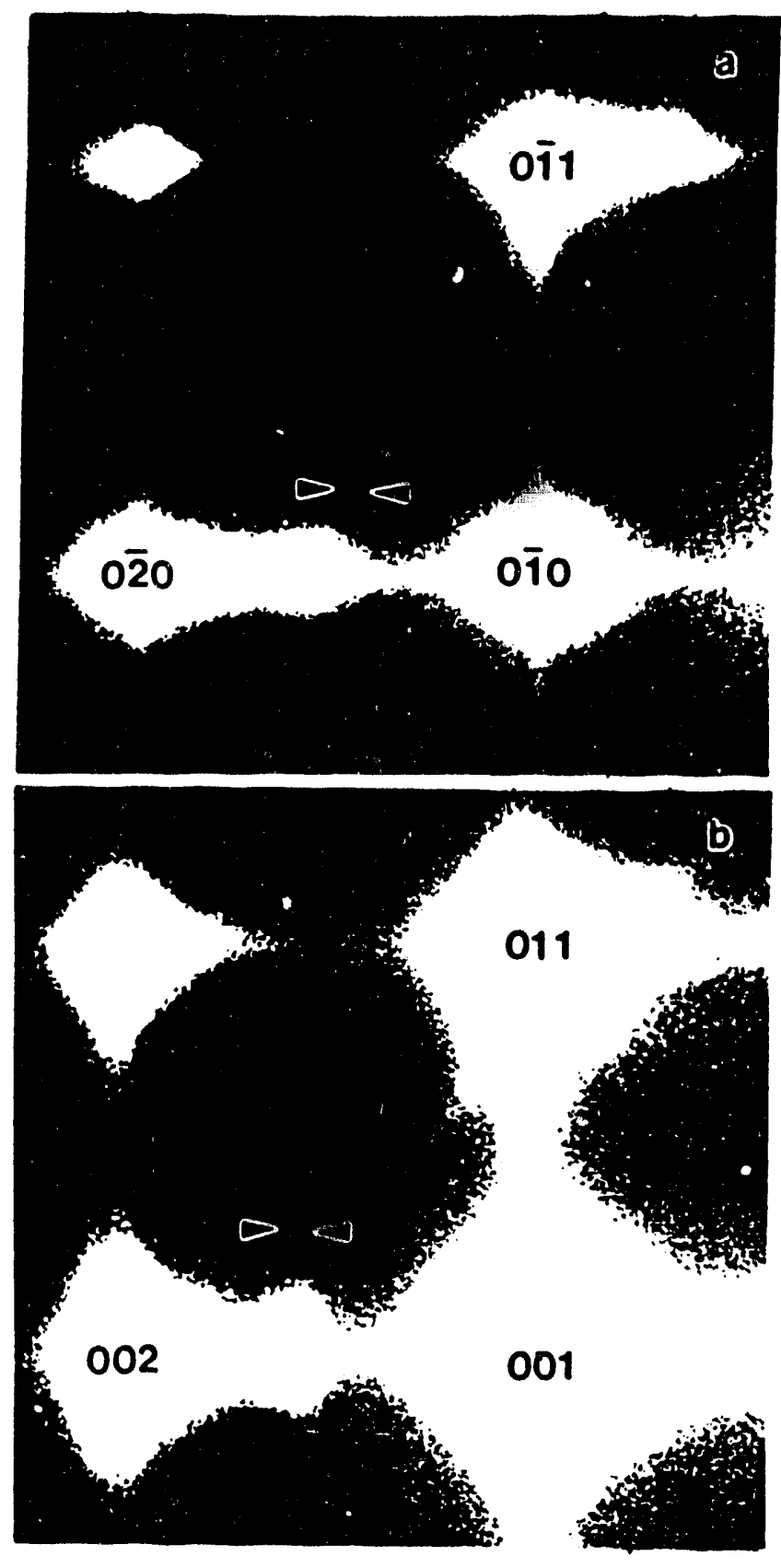

Figure 5.5: Comparison between two different kinds of streak-like intensity. (a) Enlargement of a section in Fig. 5.2a). The streak-like intensity is due to stacking faults. (b) Enlargement of a section in Fig. 5.4a). The streak-like intensity is due to thin twin domains. Notice that the streak-like intensity in a) differs in both position and direction from the one in b). Compare a) and b) with Fig. 5.6 and Fig. 5.4c), respectively. 

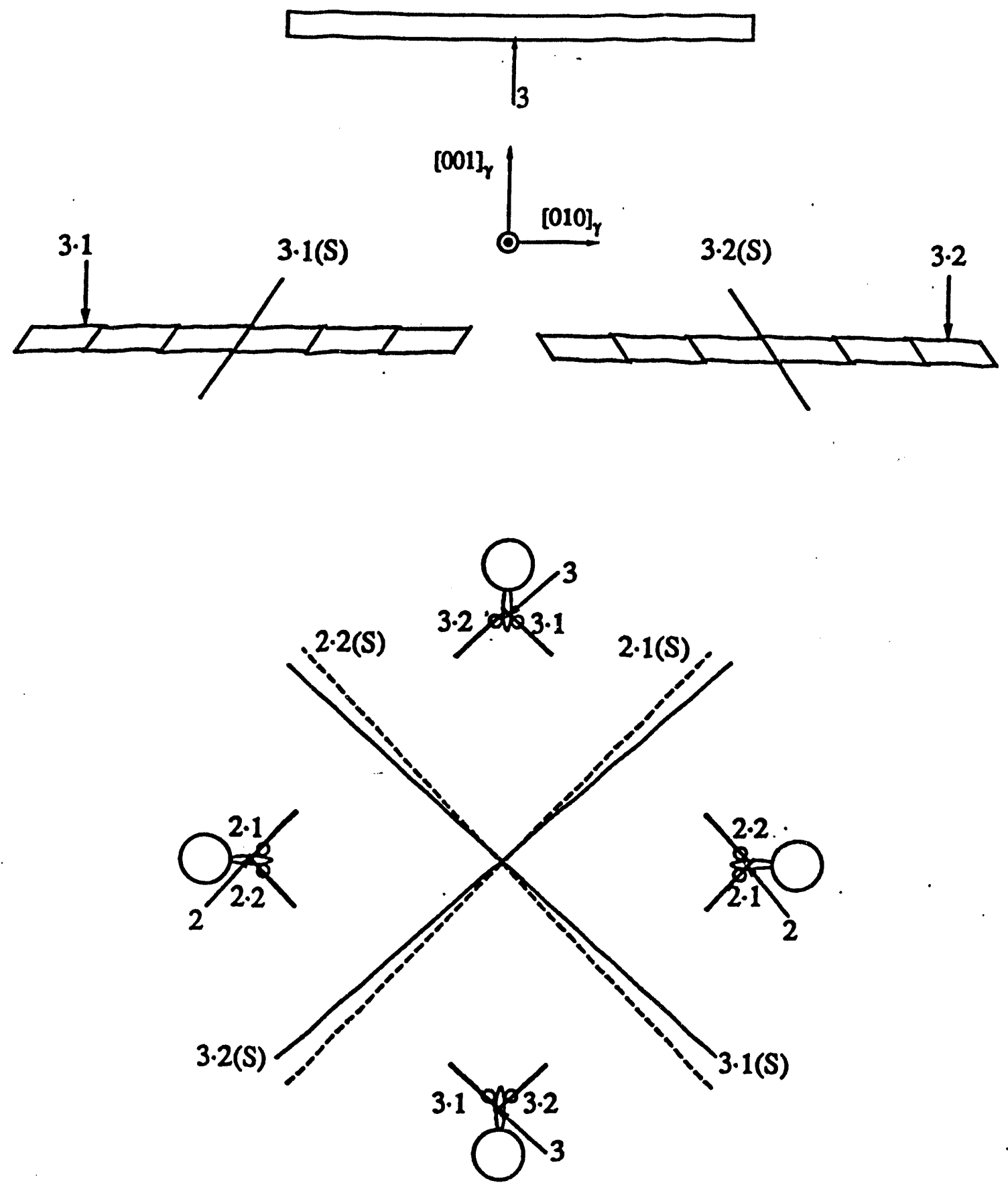

Figure 5.6: Schematic illustrating the streak-fike intensity due to stacking faults in Fig. 5.2a) and in Fig. 5.5a). 
regions in Fig. 5.4a). The schematic diagram presented in Fig. 5.6 illustrates this point (compare with Fig. 5.4C)). Fig. 5.7 shows BF and DF images which were taken from an asquenched state similar to that of Fig. 5.2 (i.e. Q1) by use of the same diffraction condition as that of Fig. 5.3. It should be noted from the DF image that in comparison with the DF image of Fig 5.3b), the contrast due to thin twin domains is now seen to be virtually non-existant in the plates of the variant 3.2. A few of the detectable thin twin domains are marked by arrows. Consistent with this finding. striation contrast prevailing in the plates of the variant 3.1 appears to be type 2 contrast rather than the contrast related to the saw-tooth morphobgy. This suggests that for the as-quenched state Q1, streak-like intensity and associated striation contrast may be ascribed not to thin twin domains but to stacking faults.

From the foregoing characterization of the polydomain (poly-twin domain and polytranslational domain or stacking faults) plates in two different as-quenched alloys, a few fundamental questions may arise; What are the possible mechanisms of formation ?' and ' do different polydomain plates form via different mechanisms ?'. With regard to these questions, a close examination of Fig. 5.3 in relation to Fig. 5.7. leads to a few interesting observations. A prominent feature, as commonly observed in both as-quenched states, is the intersection of plates. To be more specific, plates of one orientation are often intersected by plates of another orientation and in such cases, the intersecting plate is always found connected with a planar defect in the plate intersected. This can be seen, for example; near the locations indicated by arrows in Fig. 5.3b) and in Fig. 5.7a). Anather feature to note is; as the average size of the plates increases from one quenched state to another, the predominant planar defects change from stacking faults to twin domains (i.e. the width of pianar defects increases.). From these observations, the following suggestions may be offered. Firstly, intersection of plates may play an important role in the formation of either stacking faults or twin domains in an initially single domain plate. Secondly, the formation of twin domains may initiate with the formation of stacking faults through shearing process but may proceed, controlled overall by diffusional atomic transport. According to this view, the stacking faults in more rapidly quenched Q1 alloys are kinetically arrested features of the 

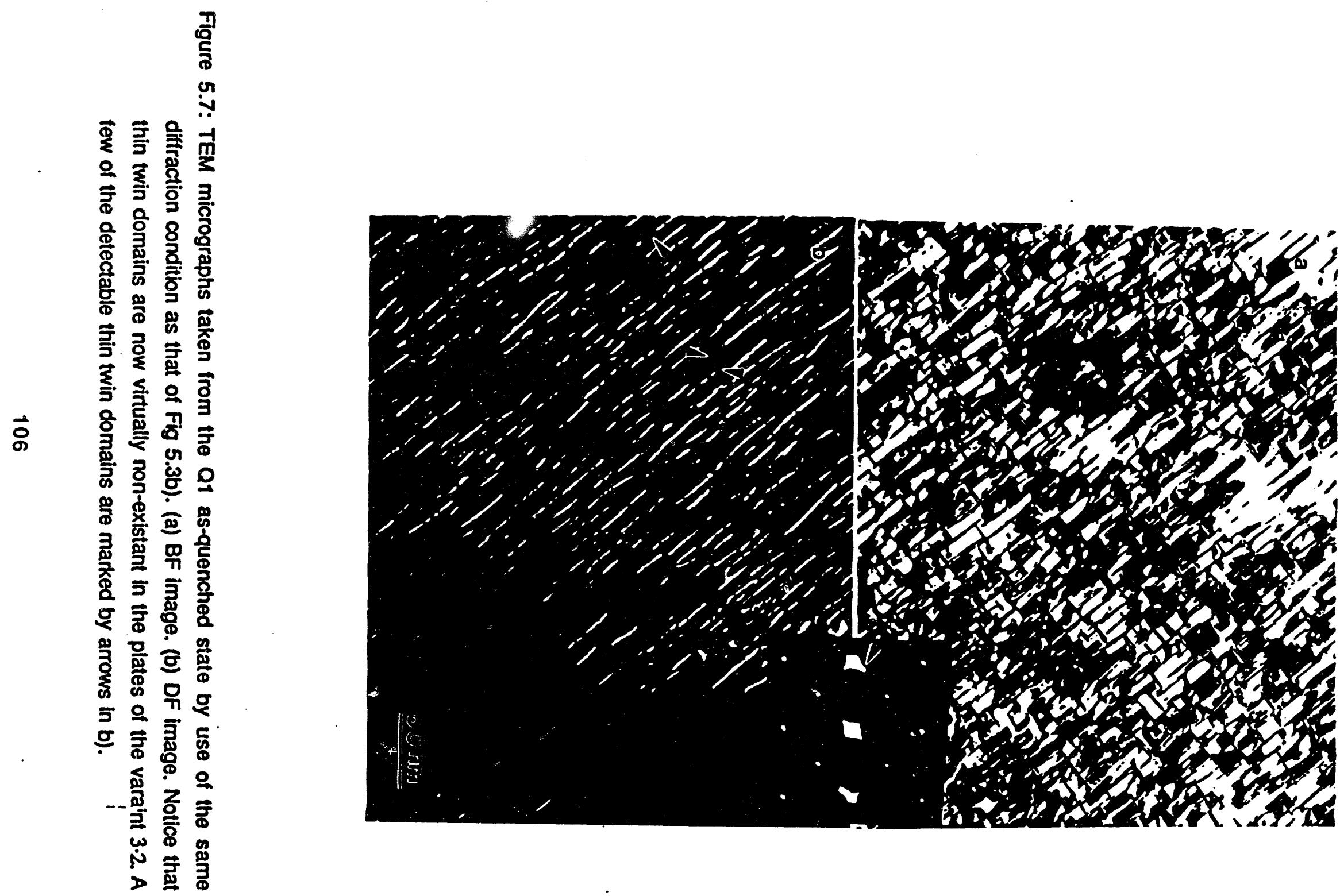
developing twin domains and thus may be regarded essentially as embryonic or underdeveloped twin domains.

\subsubsection{High Resolution TEM (HRTEM) Study of a Rapldly As-quenched State.}

In the previous subsection, we characterized the planar defects in the precipitate plates of as-quenched alloys based on Information extracted from conventional TEM. Although characterization, on its own, is self-consistent, the extremely fine nature of the defects still makes a direct visual evidence desirable, especially in view of the controversy in existing interpretations. In previous TEM studies, planar defects were interpreted to be 'quasi-eutectic $\gamma$ phase' [38] or stacking faults [39], but the possiblity of thin domains was not discussed. For this purpose and in order to gain information which may eilher support or help us detail the proposed outline of a possible formation mechanism, high resolution TEM was carried out for the as-quenched state comparable to that of Fig. 5.7.

Fig. 5.8 shows a many-beam lattice image taken exactly along the $[100]_{\gamma} z 0 n e$ axis. The $[110]_{\text {prec }}$ axis is nearly parallel to this direction. By virtue of the enhanced resolution, one can clearly see a strong correlation between the intersection of plates and the formation of planar defects. The correlation is manifested in that the average distance between planar defects is comparable to that between intersecting plates. This suggests that the separation between planar defects in a plate is determined by the spatial distribution of plates of another orientation. Intersecting plates are not detectable near some of the planar defects, however, this does not rule out the possible role of plate intersection in the formation of the planar defects. This point is illustrated, for example, by the feature marked "1". The feature, together with a noticeable non-uniformity in size and thickriess of the precipitate plates suggest that the absence of intersecting plates may be mainly due to the dissolution of the plates during the coarsening process which is under way. This may explain why intersecting plates were not frequently observed near thin twin domains in the relatively slow-quenched state shown 


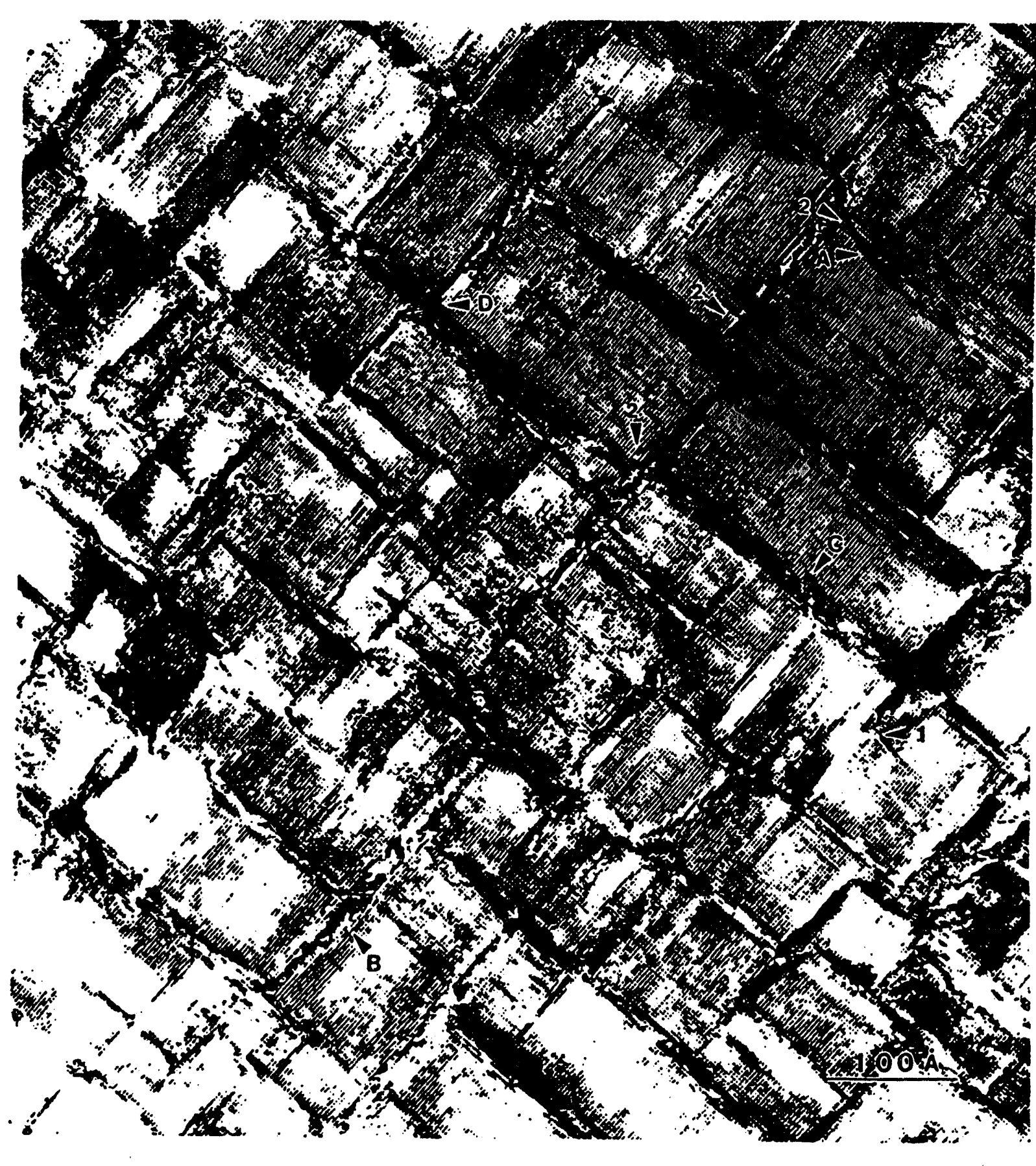

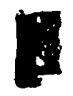
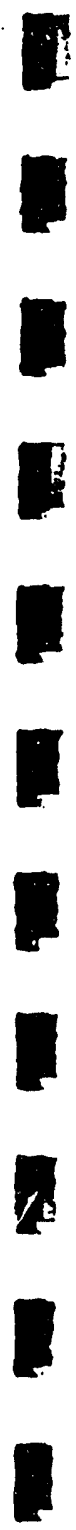

Figure 5.8: Many beam lattice image taken along $[100]_{\gamma} / 1[110]_{\text {prec }}$. Notice a strong correlation between the intersection of plates and the formation of planar defects. 
in Fig. 5.3. Where intersecting plates are observed, it is found that a plate is intersected at its broad face by either a plate of the other orientation from one side, or by two plates, one from either side. An interesting feature to note is that in the latter case, two intersecting plates are often shifted relative to each other along the broad face of an intersected plate and connected by a planar defect. It seems to be obvious that the morpinology is not likely to form without the influence of a planar defect. This is because the intersection of each plate on elther side of a broad face is a diffusionally isolated event, which should not lead to a correlation in their intersecting positions. In this regard, $t$ is interesting to note that the plates marked " 2 " gradually shift their center of thickness as they approach the associated planar defects.

Fig. 5.9 presents an enlarged viow of the region marked $A$ in Fig. 5.8. On the matrix side, Be atom columns are lacking in intensity, probably due to very small scattering power of Be atoms. Near the upper broad face of the precipitate, one can notice the continulty of lattice planes, indicating that the interface is highly coherent. What appears to be planar defects in Fig. 5.8 are marked by arrows. Although the precise nature of defects can not be determined due to blurriness of the atomic column images, it looks clear that there are lattice disturbances and they are not twins. Near these defects, the precipitate unit cells are slightly more elongated along the thicknes\$ direction with some monoclinicity. In conjunction with this, they are also slightly rotated relative to the unit cells away from defects. These may be. seen, for example, along the atomic columns indicated by two solid lines. Planar defects marked $B$ and $C$ in Fig 5.8 are shown in Fig. 5.10a) and 5.10b), respectively. Each of these planar defects is associated with a single intersecting plate. In the micrographs, circles superimposed on certain atomic columns represent the stacking sequence of (111) planes across each planar defect. By comparison with the schematic diagram shown in Fig. 5.11, these planar defects are found to be stacking faults; presumably intrinsic (B) and extrinsic (C). A planar defect associated with two intersecting plates (feature marked $D$ in Fig. 5.8 ) is shown in Fig. $5.10 \mathrm{c}$ ). One can clearly notice a thin twin region of a unit cell width. We consider that the results of Fig. $\mathbf{5 . 1 0}$ may not be taken as an indication that two intersecting 


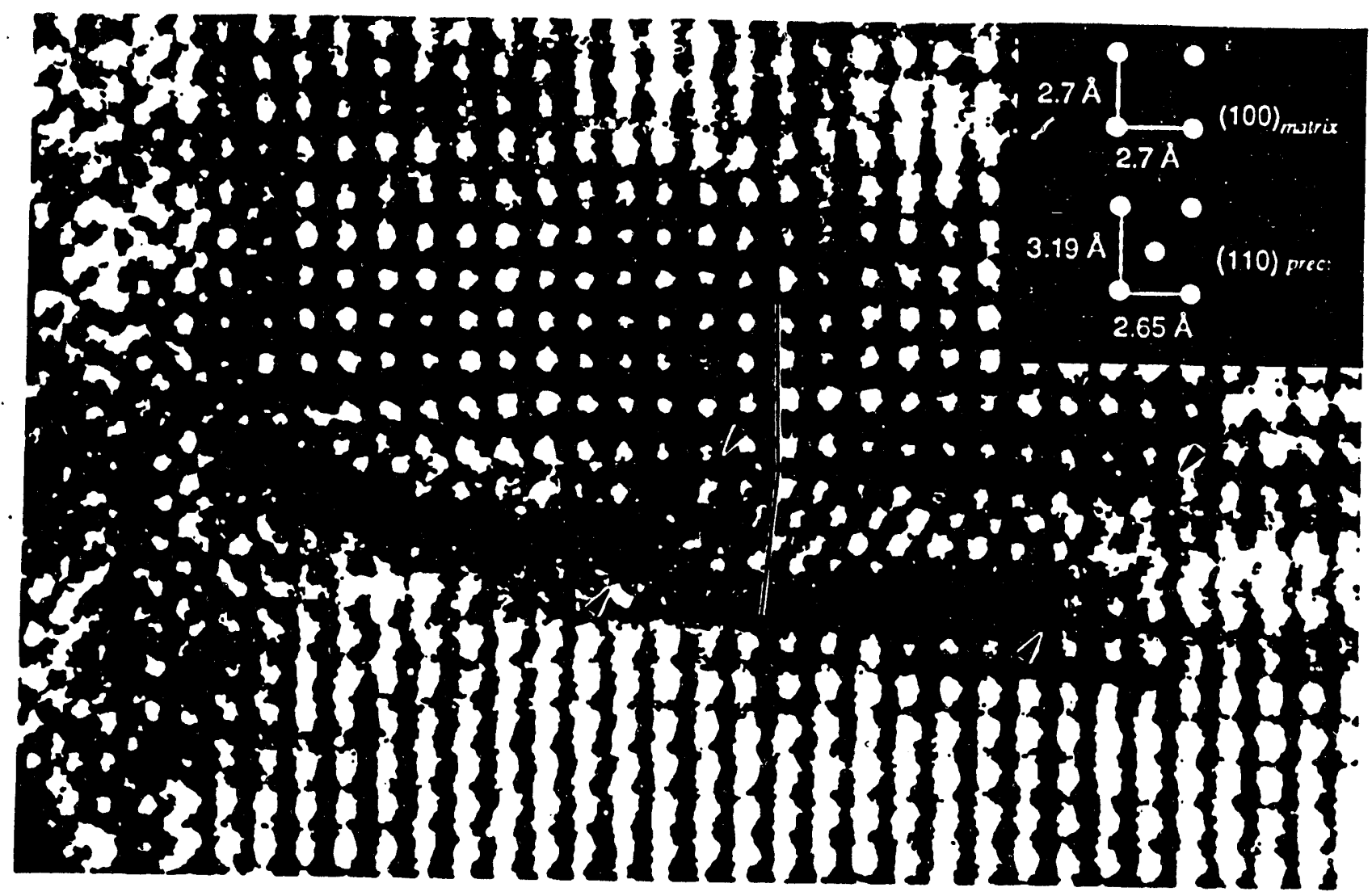

Figure 5.9: Atomic resolution image of the region marked $A$ in Fig. 5.8. Notice that near the defects marked by arrows (whose nature is no: exactly known), precipitate unit cells are not only more elongated along the thickness direction but also rotated relative to the unit cells away from defects. 


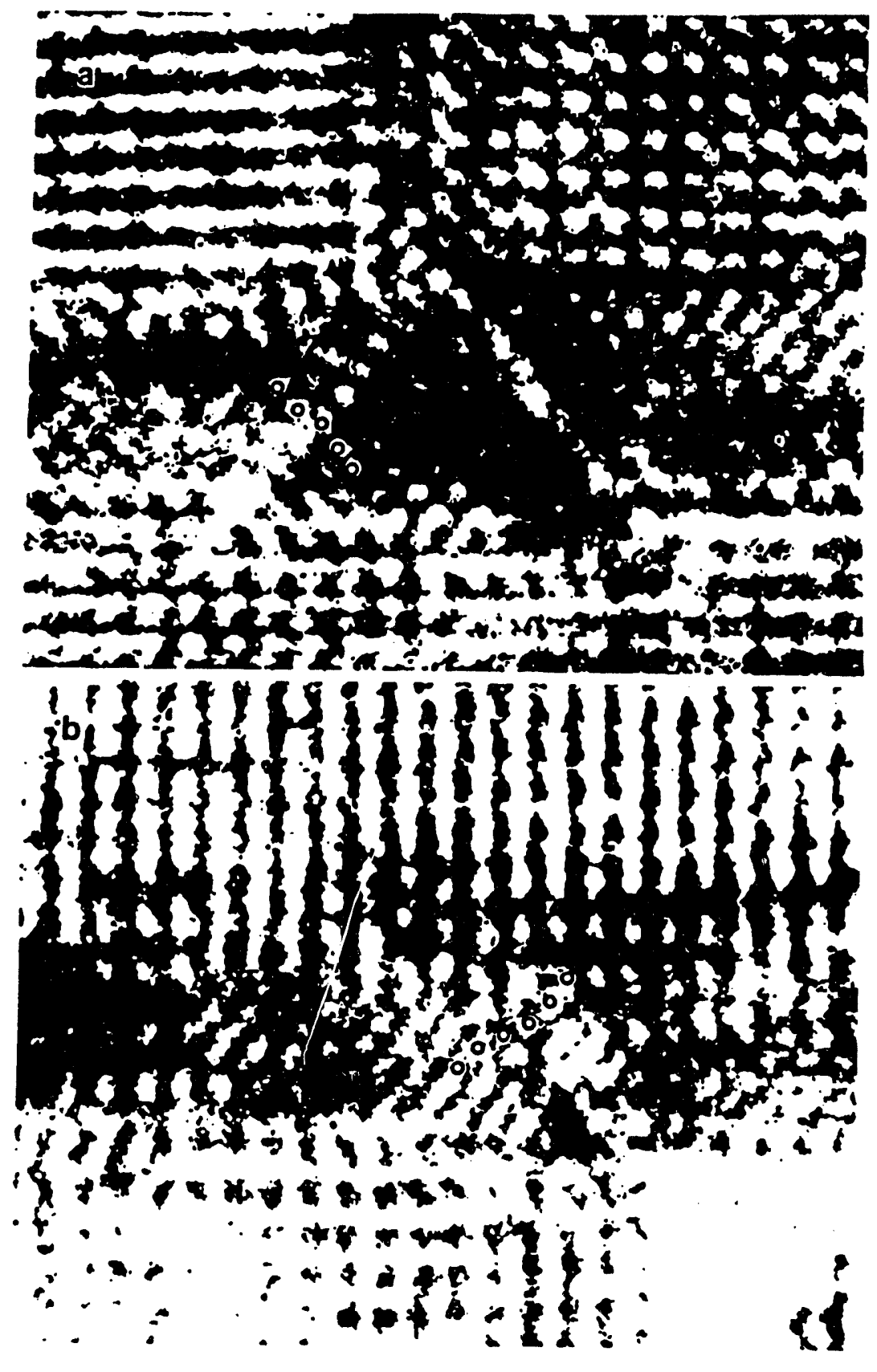

Figure 5.10: Atomic resolution images of the planar defects marked by $B$ and $C$ in Fig. 5.8. (a) $B$; intrinsic stacking fault. (b) $C$; extrinsic stacking fault. Compare with Fig. 5.11. 


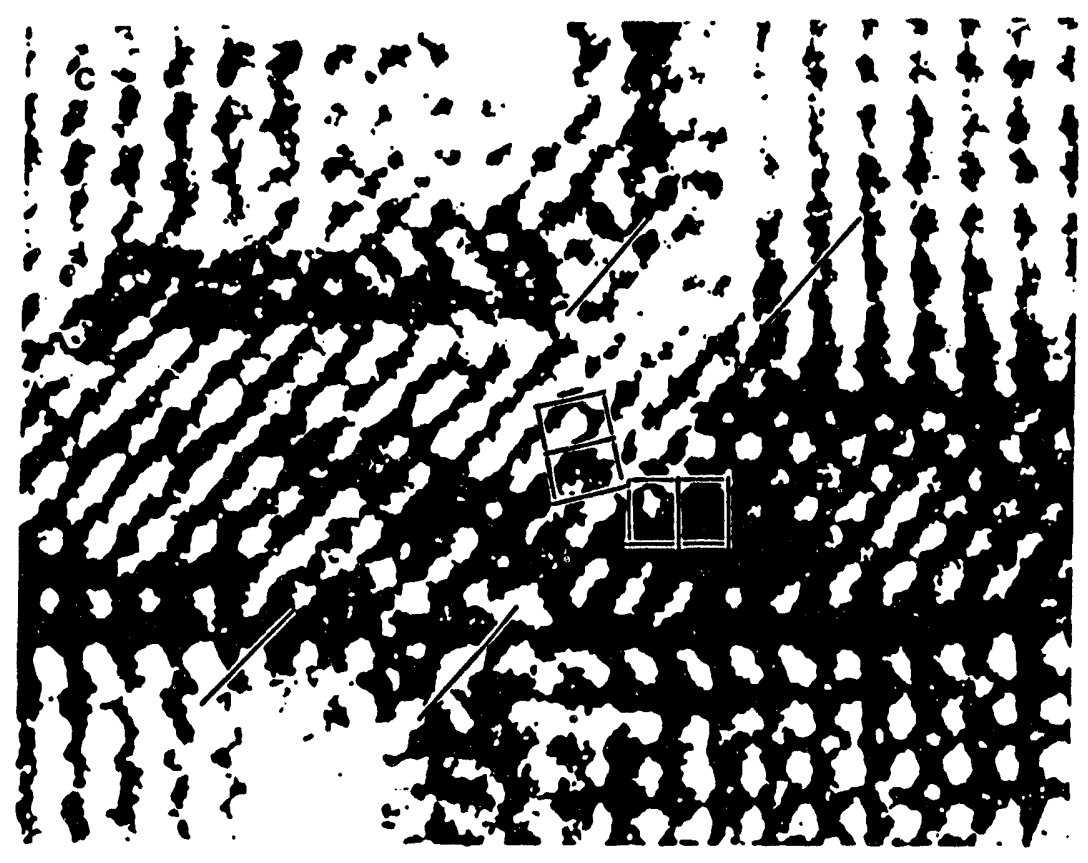

Figure 5.10: cont. (c) Atomic resolution image of the planar defect marked by $D$ in Fig. 5.8. Notice a thin twin domain of unit cell width. Compare with Fig. 5.11. 


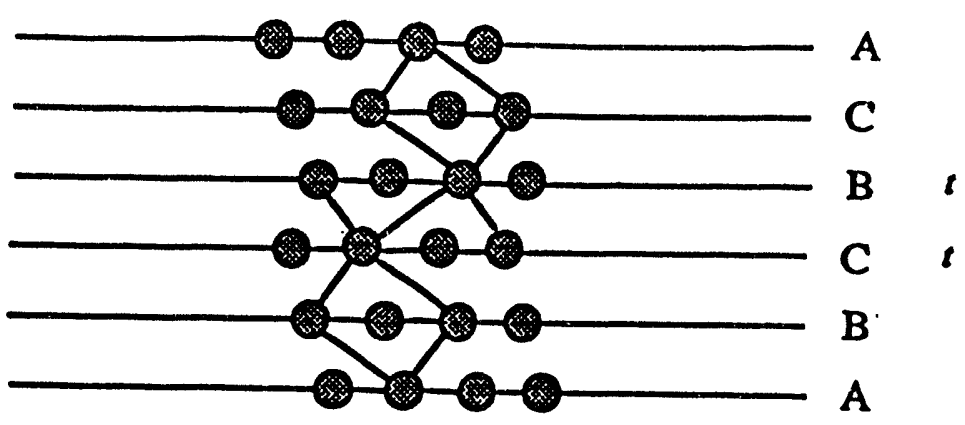

a) Intrinsic Stacking Fault

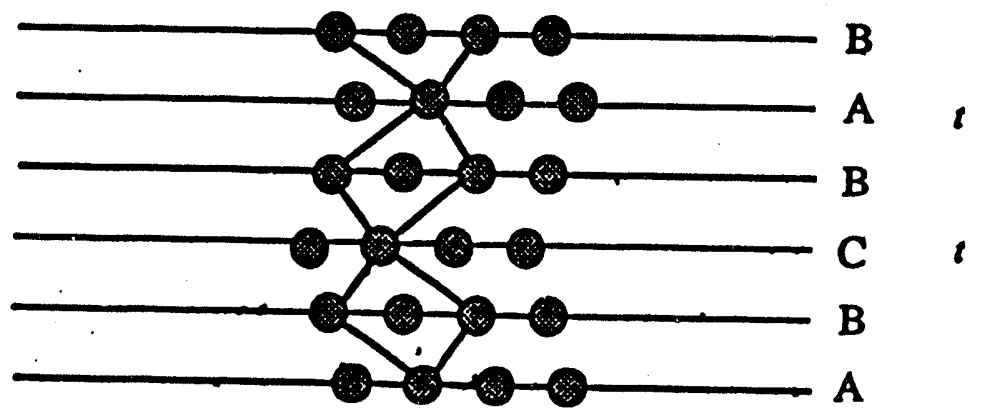

b) Extrinsic Stacking Fault

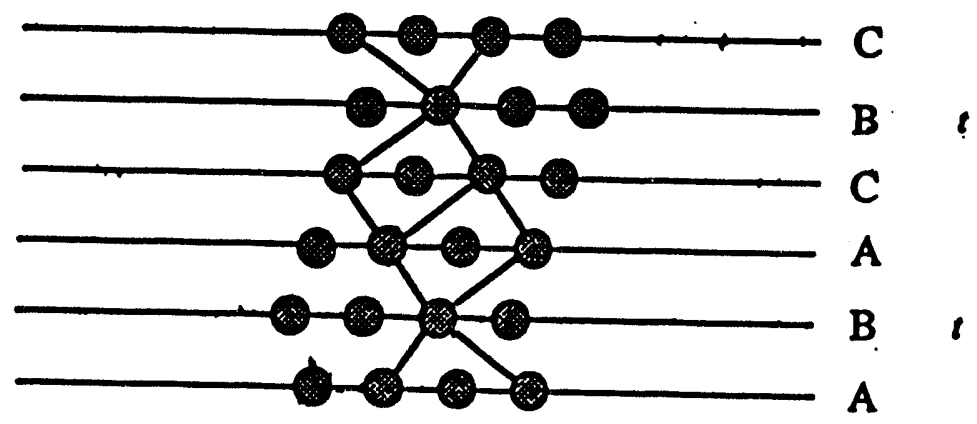

c) Twin

Figure 5.11: Schematic Illustrating the (111) stacking sequence in various planar defects. Notice that the number of twinned layers in each type of planar defect is (a) 2 , (b) 3 and (c) minimum 4. $t$ denotes the mirror plane of a local stacking sequence. 
plates are required for the formation of a twin domain. Rather, they could be taken as a suggestion that given the kinetics, the formation of a twin domain may be facilitated by the association of the two intersecting plates.

\subsubsection{Baln Straln Relaxation Toward Equilibrium.}

Based on our understanding of the as-quenched states, the later stages of the Bain strain relaxation were examined in the alloys prepared by two different heat treatments: aircooling and rapid-quenching followed by aging. As can be expected from the CuBe phase diagram shown in Fig. 1.2, the former process, in contrast to the latter, provides bcc disordered alloys with an extended reaction time of an isostructural decomposition in the (B2 + bCc) two phase field. This means that, in the course of alr-cooling, the boc precipltate plates forming at a higher temperature regime may undergo a considerable degree of coarsening prior to the onset of the Bain transformation. The question that we explored was; ' what diversification in precipltate morphology would be brought about from these different kinetic paths of the decomposition ?'.

\subsubsection{Alr-Cooled State.}

The main characteristics of the air-cooled state are depicted in a serles of TEM photographs presented in Fig. 5.12. From the BF micrograph, one can notice the $\langle 001\rangle_{\gamma}$ network of poly-twin precipitate plates which have the characteristic saw-tooth morphology. Some of the precipitate plates appear to be a single domain type, yet they are still conjugated to other plates to form a truss-like poly-twin morphology. In light of the discussion which we nave made eartier in the interpretation of our HRTEM results, it is thought that which one of these two kinds of morphology actually appears in a local region, depends strongly on the distribution of the neighboring plates of other $\langle 001\rangle_{\gamma}$ orientations during the incipient stages of the Bain transformation. It should be noted that the present saw-tooth morphology of the precipitate plates is different in an important feature from the one 


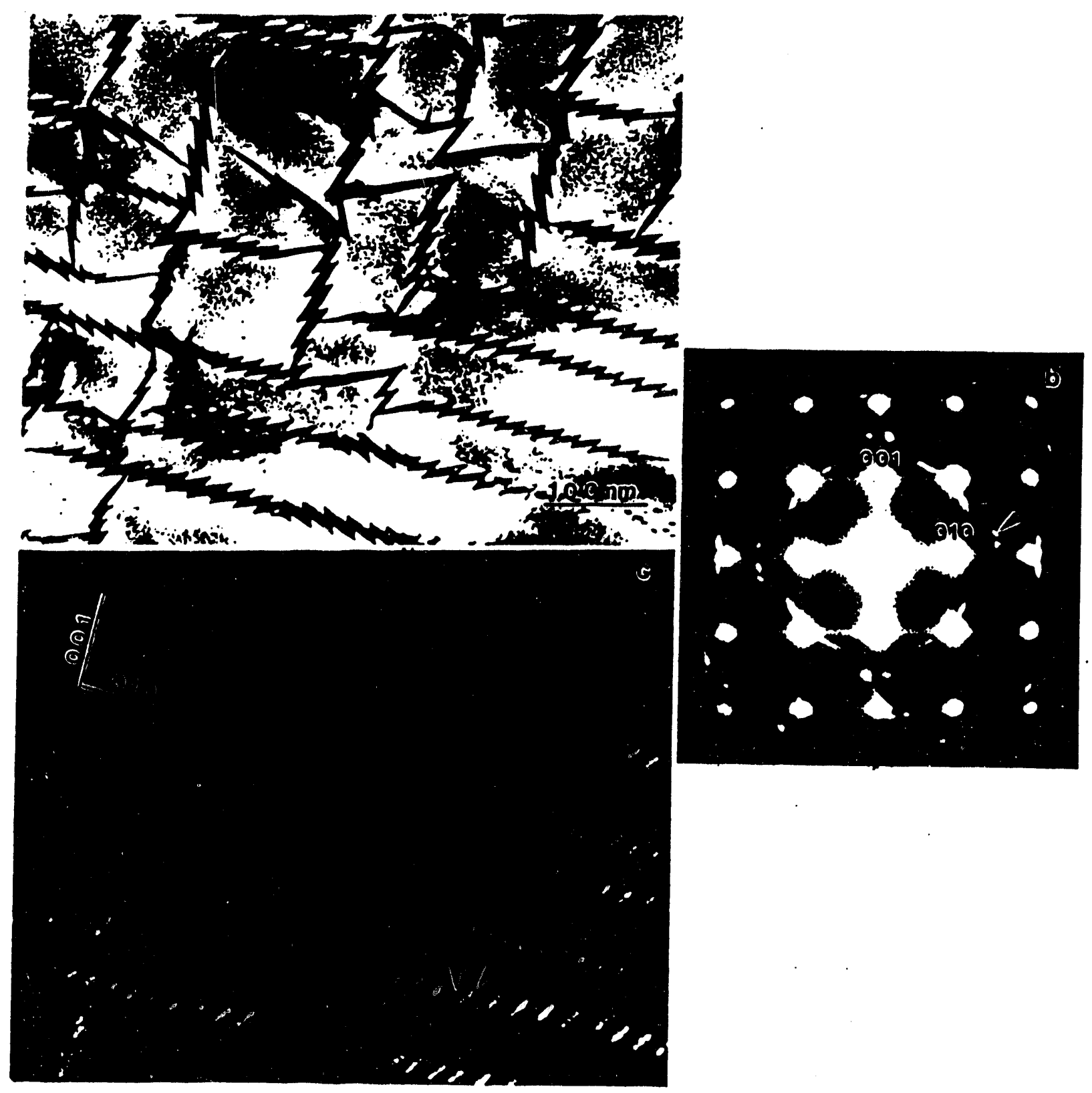

Figure 5.12: TEM photographs showing the main characteristics of the air-cooled state. (a) BF image. (b) Associated SADP taken along the $[100]_{\gamma} /\left[[110]_{p r o c}\right.$ zone axis. (c) DF image taken with the precipitate reflection indicated in (b). A region of variant $3 \cdot 1$ and that of variant $3 \cdot 1^{T}$ are marked by arrows in (c). 
observed earlier in the $Q 2$ alloy. By virtue of an extended reaction time in $(B 2+f(C)$ two phase field, the habit rotation of each domain segment is now more pronounced. From tilting experiments, It was found that the precipltate plates are edge-on along the $[100]_{\gamma}$ zone axis, meaning that the habits of the domain segments are of the (Ohk) type.

The associated SADP $\left([100]_{\gamma} / /[110]_{\text {pred }}\right.$ provides specifics of the crystallography of further Bain strain relaxation occasioned by hablt rotation. Some important quantitative features are compared with those of Fig 5.4a) and summarized in Table 5.1 for the variants 3.1 and 3.1T. These variants are marked by arrows in the DF micrograph taken with the precipitate reflection indicated in the SADP (see also the notation convention in Fig. 5.4b) and c)). The definition of each measured parameter is illustrated in the diagram inserted. For the variant 3.1, $\alpha$ and $\beta$ represent, respectively, the angle of habit rotation and the rotation angle of a (002) prec crystal lattice plane, relative to the $\alpha^{\prime}$ state characterized by the habit normal $[001\}_{r}$ For the variant 3.1 ${ }^{T}$, they simply define the coordinates of the associated $g$ vector ( $\left.=002\left(3.1^{T}\right)\right)$ with respect to the $002(\gamma)$ reflection. As one can see from the SADP (also from 5.4a)), 002 (3.1) precipitate reflections spread over a certain range of angle, reflecting the non-uniformity in the extent of habit rotation among different segments of the variant 3.1. Each of the angles listed for the variant 3.1 was measured from a position which is about half way from the center to the end of a respective angle spread. For the variant $3 \cdot 1^{T}$, they are measured for the intensity maximum. The ratio $t$, a direct measure of Bain strain relaxation, was estimated for each set of $\alpha$ and $\beta$ values. On average, the value of the aircooled state is about $4 \%$ larger as compared with that of the Q2 state but is less than the equilibrium value. This indicates that the polytwin precipitates in the given air-cooled state are still away from equilibrium (or elastically constrained). An interesting trend displayed by the listed values of the angle is schematically reproduced in Fig. 5.13 including the variant 2.1. It shows that twin domains $(3.17)$ in the $[001]_{\gamma}$-oriented polytwin plates initially have quite a different lattice orientation from that of the major segments $(2.1)$ in the $[010]_{\gamma}$-oriented polytwin plates, yet the difference gradually diminishes as the habit rotation progresses. It can be easily seen that the difference in lattice orientation between $3.1^{\mathrm{T}}$ and 2.1 may not 


\begin{tabular}{|c|c|c|c|c|c|c|}
\hline & \multicolumn{2}{|c|}{$\alpha$ (degree) } & \multicolumn{2}{c|}{$\beta$ (degree) } & \multicolumn{2}{c|}{$t=\frac{a}{\sqrt{2} b}$} \\
\cline { 2 - 7 } & $Q 2$ & Air-cool & Q2 & Air-cool & $Q 2$ & Air-cool \\
\hline variant 3.1 & 13 & 18 & 4 & 6 & 0.903 & 0.931 \\
\hline variant 3.1T & 36 & 31 & $10(?)$ & 10.5 & $0.865(?)$ & 0.9 \\
\hline
\end{tabular}

Cn $(t)$ aquil $\approx 0.9425$

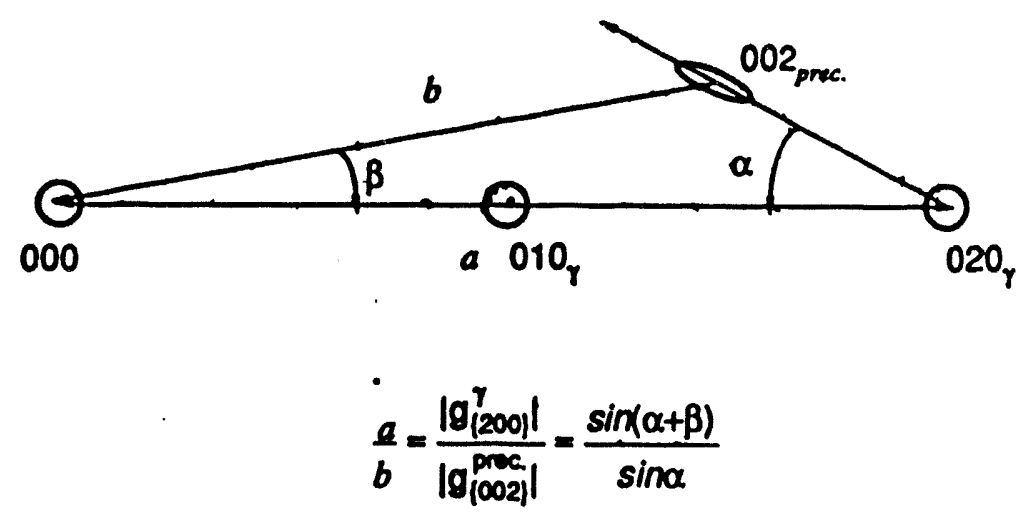

Table 5.1: Comparison between the $Q 2$ and the air-cooled state in terms of the Bain strain relaxation. The definition of each measured parameter is given in the associated schematic diagram. 


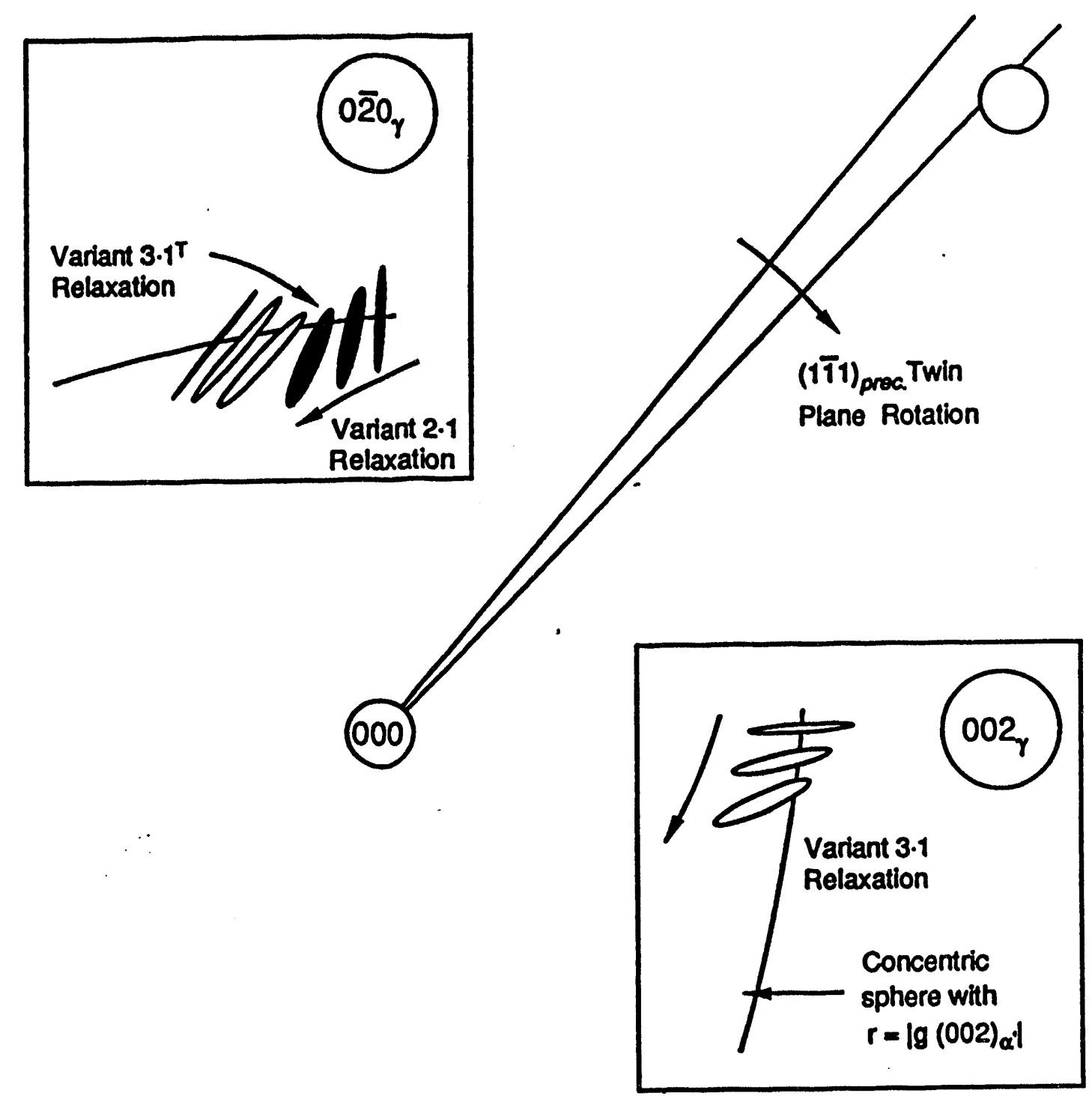

Figure 5.13: Schematic llustrating the changes in SADP with progress of the segment-wise hablt rotation of the saw-tooth type polytwin plates. Notice the gradual diminishing of the angle separation between the precipitate reflections of 002 $\left(3 \cdot 1^{T}\right)$ and $002(2 \cdot 1)$ 
vanish unless the twin plane $(1 \overline{11})_{\text {prec }}$ becomes precisely parallel to the matrix $(0 \overline{11})$ plane.

\subsubsection{Quenched/Aged State.}

The Bain strain relaxation in aged states was examined in the alloys which were quenched and aged at $300^{\circ} \mathrm{C}$ for $24 \mathrm{hrs}$. Some selected features of the microstructures of the aged alloys are shown in Fig. 5.14 and 5.15. Our attention is first given to the general appearance of the microstructures. From the micrographs of Fig. 5.15, one can notice, in contradistinction to the characteristic feature of the air-cooled alloys i.e. Well-developed sawtooth morphology, an array of individual precipitate plates with each having a respective habit plane rotated globally over the whole plate. From tilting experiments, the habit planes are found to be once again of the $(0 \mathrm{hk})_{\mathrm{y}}$ type, specifically $(03 \mathrm{t})_{\mathrm{y}}$ on average. Distinct as they are from the microstructures of the air-cooled alloys, the microstructures of the aged alloys were often found to exhibit a characteristic spatial distribution of the precipitate plates in which regions of different $c$ axis variants are interweaved to form a macroscopic polytwin configuration. This is shown in the BF images of Fig. 5.15. It is noticed that, as in the case of a polytwin plate, the boundaries between different regions run nearly parallel to the $\langle 110\rangle_{\gamma}($ $-<111\rangle_{\text {prod }}$ directions. As is well-known from studies of the tweed microstructure [87], such disintegration into different elastic colonies is a manifestation of the tendency to minimize the total elastic energy of a two phase system and is caused by a strain-induced interaction among precipitate plates in the course of coarsening.

As for the details of the individual precipitate plates, a few interesting aspects are found from comparison of Fig. 5.14 with Fig. 5.15 (each set of TEM photographs were taken under a similar diffracting condition.). Firstly, striation contrast inside the plates is observable in the micrographs of both sets, yet it is more prominent in the latter. In particular, much of striation contrast in Fig. 5.15a) appears to be characteristic of thin twin domains. In association with this, one can notice from the SADP's sharply streaked intensity distribution near the 002 precipitate reflections. According to the diagrams of Fig. 5.4b) and Fig. 5.6, such streaked 


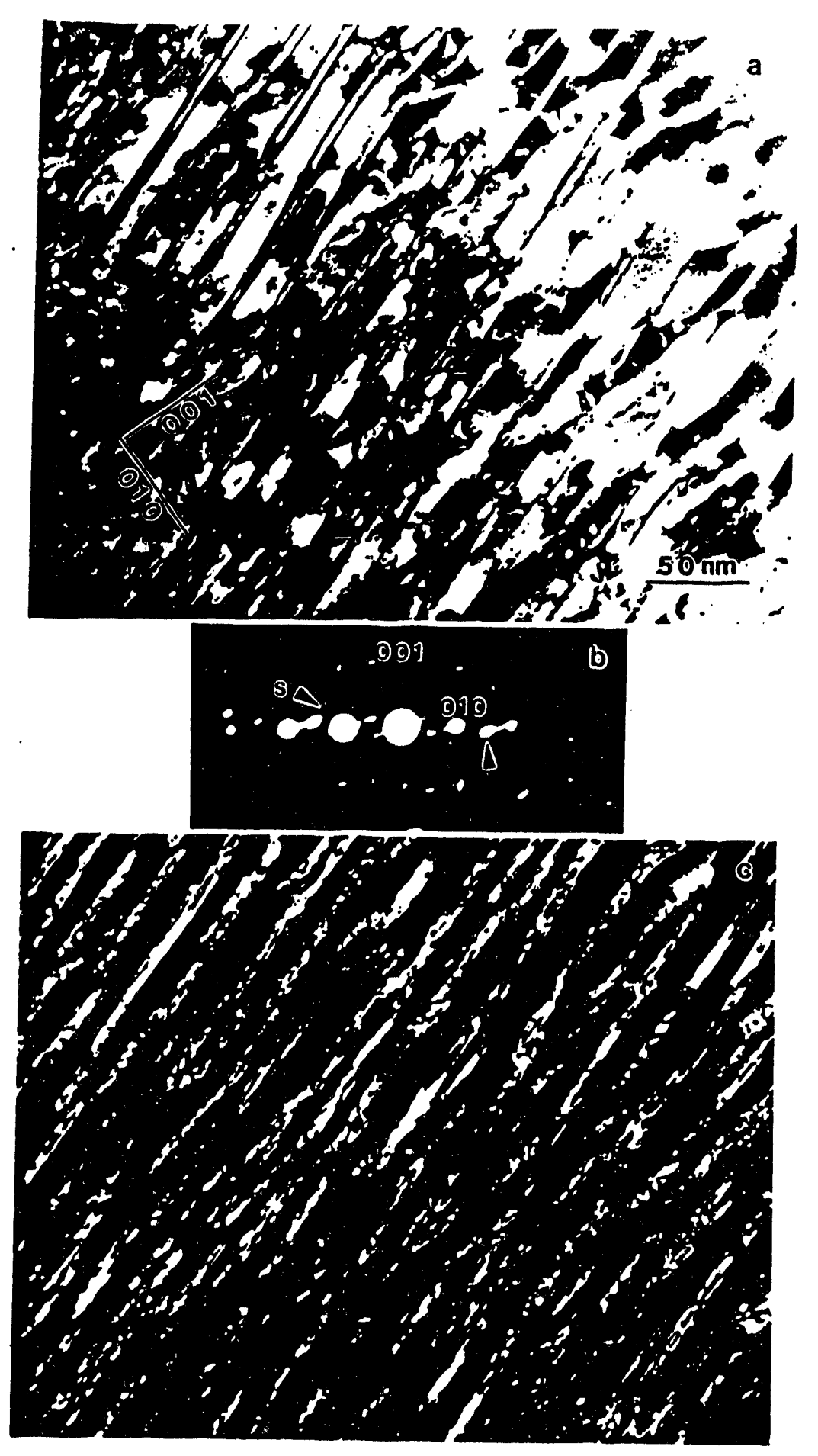

Figure 5.14: TEM photographs taken from an alloy aged at $300{ }^{\circ} \mathrm{C}$ and for $24 \mathrm{hrs}$. (a) BF image. (b) Associated SADP. (c) DF image taken with the 002 precipitate reflection indicated in (b). Notice, in contrast to the saw-tooth morphology of the air-cooled alloys, individual precipitate plates with each having a respective habit plane globally rotated over the whole plate. 


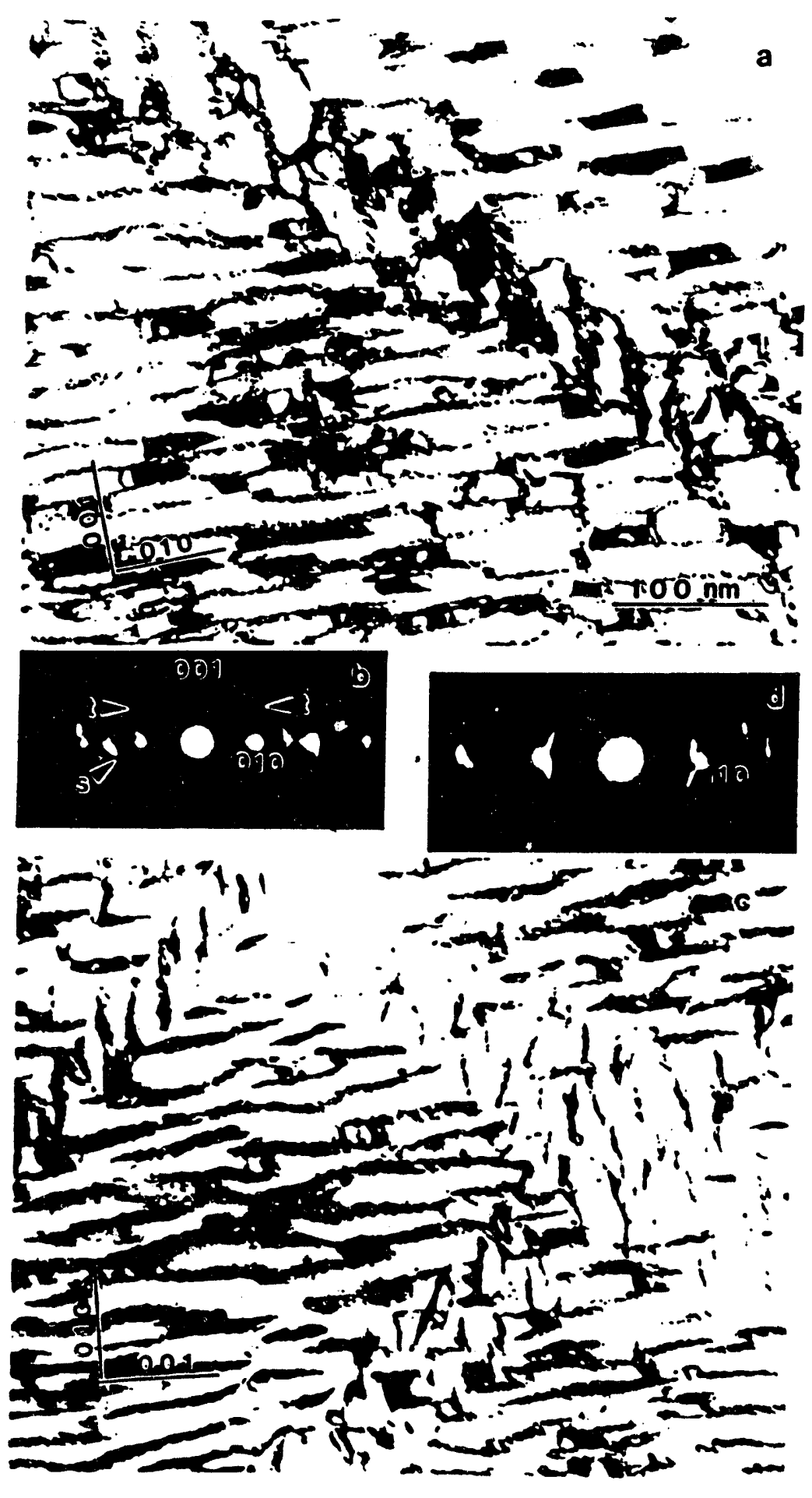

Figure 5.15: TEM photographs taken from an alloy aged at $300^{\circ} \mathrm{C}$ and for $24 \mathrm{hrs}$. (a) and (c) BF images. (b) and (d) Associated SADP's. Notice a characteristic spatial distribution of the precipitate plates forming a macroscopic polytwin configuration. 
intensity may be ascribed to the stacking faults (s) in the case of Fig. 5.14b) whereas to the stacking faults (s) and thin twin domains (t: $2.1^{\mathrm{T}}$ and $\left.2.2 \mathrm{~T}\right)$ in the case of Fig. 5.15b). Secondly, rotation of the habit plane and thus of crystal lattice are more progressed in the case of Fig. 5.14. Measurement of the angle $\alpha$ (as defined in Table 5.1) shows that rotation of the 020 precipitate reflection is about $5^{\circ}$ smaller in Fig. 5.15b) than in Fig. 5.14b). From these observations, the following suggestion may be made: the habit plane rotation of an individual presipltate plate and concomitant coarsening may involve the elimination of the twin domains which possibly resulted from the intersection of plates during eartier stages of coarsening. In view of the long aging time employed, fine nature of twin domains (Fig. 5.15a)) and lack of detectable associated contrast in the pronouncedly rotated precipitate plates (Fig. 5.14) may not be explained unless the formation of twin domains were conflicting with the global rotation of the habit plane of the plate. We will give some detailed consideration of this in section 5.3.2.2.

\subsection{Discussion}

\subsubsection{Earllest Stages Decomposition; Stages of Passive Relaxation of the Bain Strain}

As initially noted by Tadaki et al. ${ }^{[38]}$ and Auvray ${ }^{[39]}$, the earliest stages decomposition of the hypereutectoid $\beta$ phase have rapid kinetics and a conventional water-quenching always leads to the formation of two phase microstructures which consist of the B2 ordered phase and Cu-rich coherent plate-like precipitates with average habit planes close to $\{001\}_{r}$ The overall morphology of the microstructures is typical of an isostructural decomposition in a cubic alloy except for some striation contrast inside the precipitate plates. One of the interesting features of the microstructures was the lack of APB contrast in the B2 matrix. This feature, together with the $\{001\}_{\gamma}$ habit planes of the precipitates, led us to presume that the earliest stages of decomposition of the $\beta$ phase may proceed as follows:

(1) bcc to $\mathrm{B2}$ ordering. 
(2) isostructural decomposition leading to the formation of $\{001\}_{\gamma}$ Cu-rich G.P. zones at the APB's of the B2 matrix.

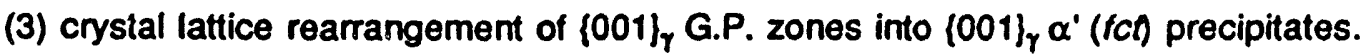

The thermodynamic and kinetic origins of the suggested decomposition path and the Bain strain relaxation along this path, are discussed below.

In Fig. 5.16a), a portion of the CuBe phase diagram is schematically reproduced with relevant lines added and/or extrapolated. The critical line of the second order A2 $\rightarrow$ B2 ordering transition is depicted as a dashed line. It is shown to form a critical end point [73] at which $\beta /(\beta+\gamma)$ and $\beta /(\gamma+\gamma)$ two phase boundaries intersect with the line of $A 2 \rightarrow B 2$ ordering. The dotted lines demarcate the conditional spinodals in which spinodal decomposition is thermodynamically possible contingent upon $A 2 \rightarrow B 2$ ordering [94-97]. As the temperature decreases, a branch of the spinodal lines merges into the line of A2 $\rightarrow B 2$ ordering $[24,97]$ as shown in the diagram. Fig. 5.16b) represents a chemical free energy vs. composition diagram which is consistent with the phase diagram characteristics at an arbitrary temperature $T_{1}$ below the eutectoid point. The free energy of the XB2) phase plotted in the diagram is assumed to be such that for a given composition, it satisfies the extremization condition; $\left.\frac{\partial F}{\partial \eta}\right|_{k}=0$. According to the diagrams, a disordered $\beta$ phase of the composition $c^{\beta}$, if supercooled to the temperature $T_{1}$, would decompose along the path $A \rightarrow B \rightarrow C \rightarrow D$ to produce the equilibrium foc precipitates.

\section{Congruent Ordering: $A \rightarrow B$}

Being unstable with respect to the fluctuations of long range order, the disordered phase transforms to the homogeneous B2 ordered phase without change in composition (congruent ordering). Since there is no need of long range volume diftusion, the kinetics of this process is thought to be extremely fast. Using hypo-eutectoid and eutectoid $\beta$ phase alloys (eutectoid composition: 5.11 wt.\%Be or 31.5 at.\%Be). Tyapkin et al. [98.99] and Auvray [39] examined the earliest stages of decomposition. By virtue of the extended stability (or 


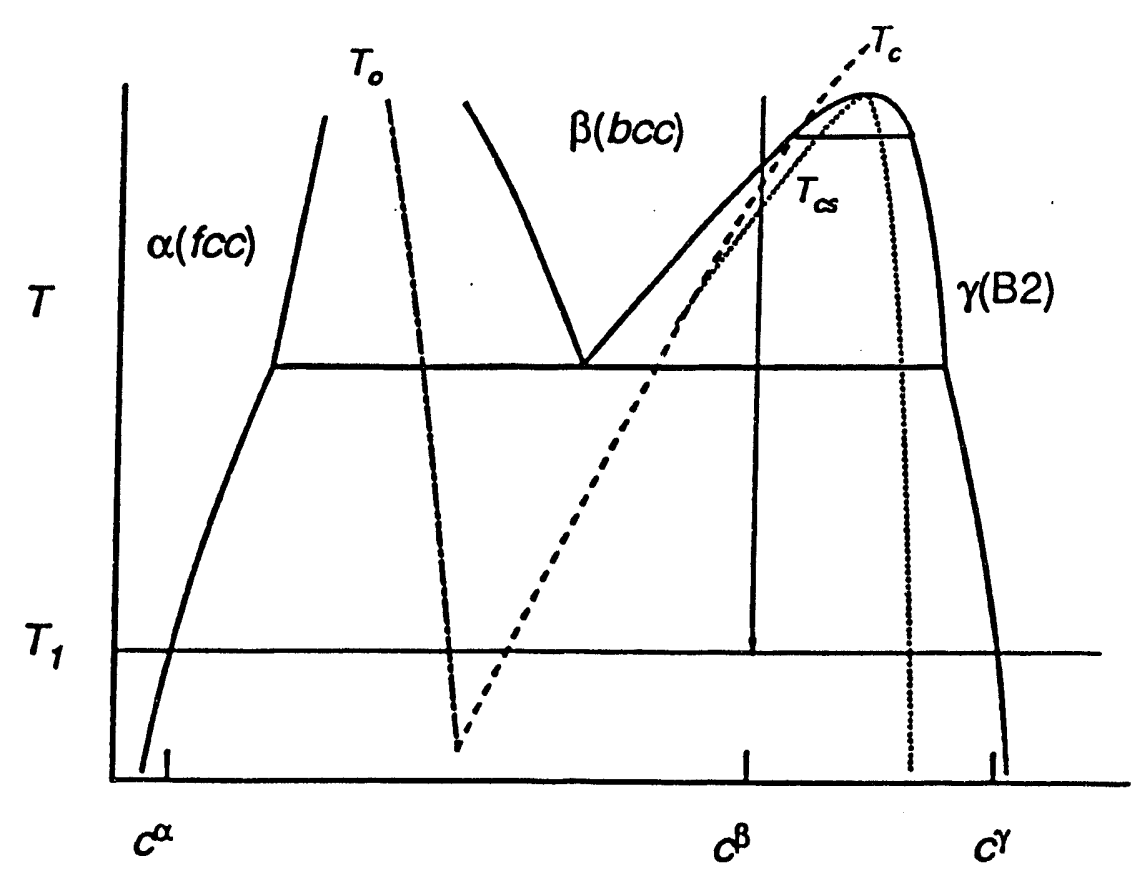

a)

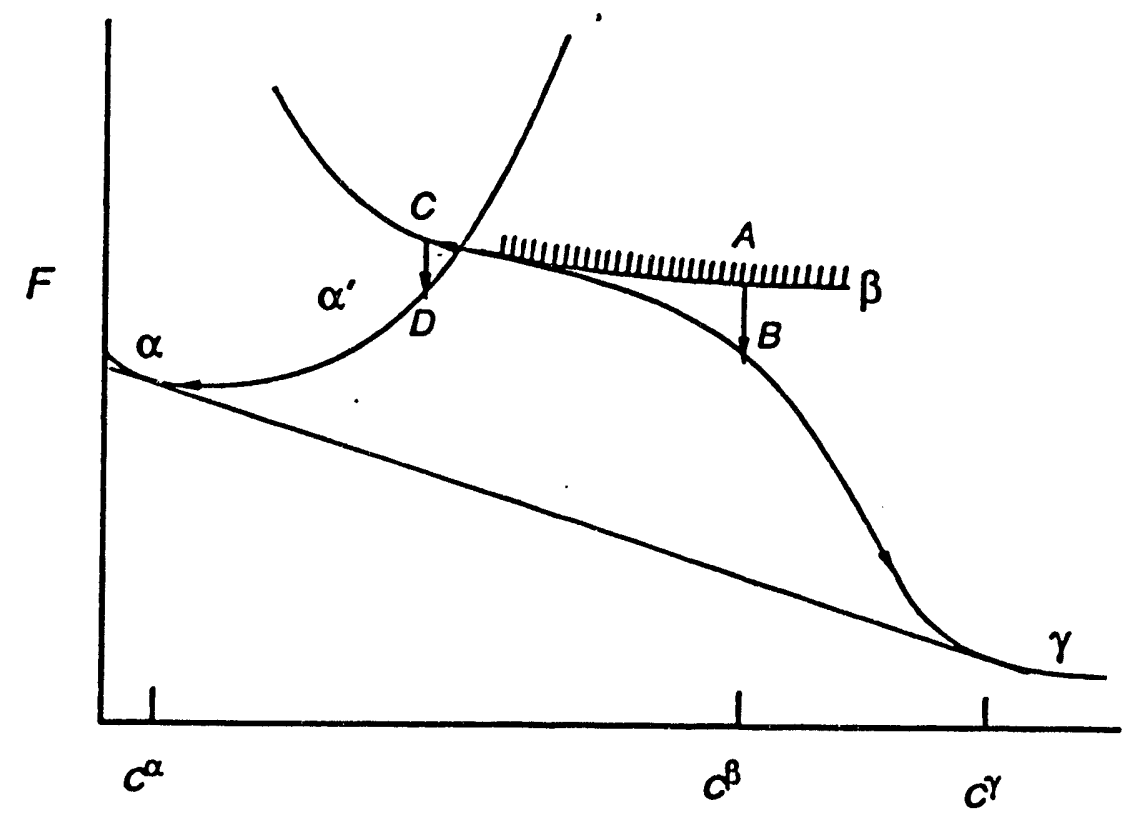

b)

Figure 5.1.6: (a) Schematic of the CuBe phase diagram. See text. (b) Chemical free energy vs. composition diagram consistent with the phase diagram characteristics at an arbitrary temierature $T_{1}$. When supercooled to $T_{1}$, a disordered $\beta$ phase of the composition, $c^{\beta}$, would decompose along the path $A \rightarrow B \rightarrow C \rightarrow D$ to prodives tine equilibrium foc precipitates. 
metastability) of the $\beta$ phase in these alloys, they were able to observe the initial ordering reaction. Based on diffraction studies, it was suggested that the disordered $\beta$ phase initially orders to the metastable $\mathrm{Cu}_{2} \mathrm{Be}\left(\beta^{m}\right)$ ordered phase which has a unit cell three times as large as that of the CuBe (B2) ordered phase with the CuCuBeCuCuBe stacking sequence on successive $(200)_{b c c}$ planes. We believe that the $\mathrm{Cu}_{2} \mathrm{Be}$ type ordering would not occur in hyper-eutectoid alloys, particularly in alloys of compositions far from the eutectoid composition. This is because the $\mathrm{Cu}_{2} \mathrm{Be}$ structure is a non-special point ordered structure in the bcc lattice. Unlike a special point ordered structure, the stability of a non-special point structure depends strongly on the change in interatomic potential with composition and temperature, and hence results in a very limited region of stability (or metastability) in the $T-c$ diagram. [24.28]

Isostructural Secondary Decomposition: $B \rightarrow C$

Since it is inside the region of the conditional spinodal, the non-stoichiometric B2 ordered phase resulting from the congruent ordering is unstable with respect to long wavelength concentration fluctuations. When the average APD size of the B2 phase is much larger than the wave length of the concentration fulctuations, this would lead to a continuous phase separation of the B2 ondered phase by the spinodal mechanism. In the presence of a large density of APB's, however, the spinodal mechanism may not represent a viable path for the decomposition. The APB's introduce local pertubations (or inhomogeneities) in the atomic order and, therefore, also in concentration, which otherwise, need to develop in the unperturbed ordered matrix for decomposition to occur. Obviously, this would give precipitation on APB's a kinetic advantage over any homogeneous mode of decomposition. The important role of APB's during the secondary decomposition was vividly derronstrated in a number of experimental investigations $[94.100 .101]$ and in kinetic simulation studies recently conducted by Chen and Khachaturyan [102].

As a consequence of the isostructural secondary decomposition, $\mathrm{Cu}$-rich $\{001\}_{\gamma}$ plate-like 
G P. zones exist within the Be-enriched $B 2$ matrix. The plate shape and the $\{001\}_{\gamma}$ habit of the G.P. zones are a manifestation of the minimization of the elastic strain energy which derives from the difference in composition-dependent lattice parameters between the G.P. zones and the matrix $\mathrm{B} 2$ phase. $^{\dagger}$ It is emphasized that the crystal lattice of the G.P. zones is no longer cubic but is tetragonally elongated along the direction of the habit plane normal since it is subject to the coherency constraint along the $\{001\}_{y}$ habit planes. This is shown by eq. (5.1) which is derived from eq. (2.48).

$$
\frac{a(3)_{G . P .}}{a(1)_{G . P .}} \cong \frac{a(3)_{G . P .}}{a(1)_{\gamma}}=1+\varepsilon_{0}\left(1+\frac{2 C_{12}}{C_{11}}\right)
$$

where $a$ (1) and $a$ (3) denote the constrained lattice parameters along [100] and [001] directions, respectively and $\varepsilon_{0}$ is the stress free dilatational strain defined by

$$
\varepsilon_{0}=\frac{d h}{a d c}\left(c G P_{0}-c_{\gamma}\right)_{1} \text {. }
$$

where $c$ denotes the $\mathrm{Cu}$ concentration. Due to the lack of necessary data (i.e. compositiondependent lattice parameters and elastic constants of bcc (or B2) phase), an estimation of eq. (5.1) could not be made. It may suffice to point out that this tetragonal elongation of the G.P. zone lattice has the consequence of a partial pre-relaxation of the Bain strain which will result from the bcc (or B2) to fcc crystal lattice rearrangement of the following stages.

\section{Crystal Lattlce Rearrangement: $C \rightarrow D$.}

The $\{001\}$ G.P. zones resulting from the secondary decomposition undergo the Bain crystal lattice rearrangement, as they continue to be enriched in $\mathrm{Cu}$ content. Being subject to the coherency constraint, this transition leads to the $\alpha^{\cdot}(f c t)$ precipitates with $\{001)_{\gamma}$ habit planes. The tetragonality of the constrained lattice of the $\alpha^{\prime}$ precipitates is given from eq. (2.48) by

$$
\frac{a(3)_{\alpha}}{a(1)_{\alpha}} \equiv \frac{a(3)_{\alpha}}{\sqrt{2} a(1)_{\gamma}}=\frac{1}{\sqrt{2}}\left(1+\varepsilon_{33}^{0}+\frac{2 C_{12}}{C_{11}} \varepsilon_{11}^{0}\right)
$$

$t$ According to the estimation made in [34). the elastic anisotropy of $B 2$ phase is negative $\left(C_{11}-C_{12}-2 C_{44}<0\right.$ where $C_{i j}$ 's are the Voigt notation of elastic constants), and thus $\{001\}$ habit plane is stable. 
where $a(1)$ and $a(3)$ denote the constrained lattice parameters along [100] and [001] directions of the precipitate lattice, respectively and $\varepsilon_{11}^{\circ}, \varepsilon_{33}^{\circ}$ are the stress free transformation strains defined by

$$
\varepsilon_{11}^{0}=\frac{a_{\alpha}-\sqrt{2} a_{\gamma}}{\sqrt{2} a_{\gamma}}, \quad \varepsilon_{33}^{\circ}=\frac{a_{\alpha}-a_{\gamma}}{a_{\gamma}}
$$

where $a$ 's denote the lattice parameters of the stress free $\alpha$ and $\gamma$ phases with compositions of $\alpha^{*}$ and $\gamma$ phases. An estimation of eq. (5.3) was made by use of the lattice parameters of the fcc pure Cu crystal $(a=3.612 \mathrm{~A})$ and equilibrium B2 phase $(a=2.7 \AA)$ and elastic constants of the pure $\mathrm{Cu}\left(C_{11}=1.684 \times 10^{12} \mathrm{dynd} / \mathrm{cm}^{2}\right.$ and $C_{12}=1.214 \times 10^{12} \mathrm{dyne} / \mathrm{cm}^{2} \mathrm{~J}$. This leads to $\frac{a(3)_{\alpha}}{a(1)_{\alpha}}=0.89$, which is about $6 \%$ larger than the tetragonality value $(=0.84)$ measured from Fig. 5.2a). ${ }^{1}$ Within the context of the theory used, this discrepancy can be ascribed directly to the use of the lattice parameters for $c_{\alpha^{\circ}}=1$ and $c_{\gamma}=0.5$, that is to say, an upper bound condition. This, in turn, suggests that the composition of the precipitates shown in Fig. 6.2a) remains away from that of the equilibrium state.

\subsubsection{Bain Strain Relaxation During Post- $\alpha^{\cdot}$ Stages}

\subsubsection{Instabllity of the $\alpha^{\cdot}$ State and Subsequent Relaxation.}

The $\alpha^{\prime}$ precipitate which has the $\{001\}_{\gamma}$ habit plane inherited from the $\{001\}_{\gamma}$ G.P. zone is unstable with respect to further relaxation of the Bain strain by an infinitesimal amount. This can be demonstrated most explicilly by use of the habit stability condition which we derived in eq. (2.42). According to the equation, a plate-like precipitate with $\{001\}_{\gamma}$ habit plane is unstable in the following condition is met:

$$
\frac{\varepsilon_{33}^{0}}{\varepsilon_{11}^{0}}<1+\frac{\xi}{2}\left(1+\frac{2 C_{12}}{C_{11}}\right)
$$

where

\footnotetext{
It is emphasized that the measured tetragonality value is not relerred precisely to the $1001 / \alpha$ state but to a near $1001)^{\prime}$ state in which the Bain strain relaxation is also achioved by introduction of stacking faults. The contribution from the stacking faults, however, would be probably very small in view of their sparse density.
} 


$$
\xi=\frac{C_{11}-C_{12}-2 C_{44}}{C_{44}}
$$

The right hand side of the equation has the absolute minimum value $(=-2)$ in the limiting case of $C_{12} \rightarrow C_{11}$. This establishes a useful criterion, namely that a plate-like precipitate with \{001\} $\}_{\gamma}$ habit plane is elastically unstable regardless of elastic constants, if $\frac{\varepsilon_{33}^{0}}{\varepsilon_{11}^{0}}<-2$. Estimation of the $\frac{\varepsilon_{33}^{\circ}}{\varepsilon_{11}^{\circ}}$ ratio by use of the lattice parameter of pure $\mathrm{Cu}$ and that of the equilibrium B2 phase gives $\frac{\varepsilon_{j 3}^{\circ}}{\varepsilon_{i 1}^{\circ}}=-6.25$, which is much smaller than the minimum value for the stability. As can be expected from eq. (5.4), use of actual lattice parameters would not yield much change in the ratio. Fig. 5.17 shows the 3-D plot of the elastic strain energy of a constrained fcc plate-like precipitate as a function of habit plane normal. Consistent with the result of the foregoing analysis, one can see clearly the local maximum of the elastic strain energy along the $[001]_{\gamma}$ direction.

As a consequence of the instability of the $\alpha^{*}$ state, further relaxation of Bain strain immediately sets in, contingent upon the transition of the $\{001\}_{Y}$ G.P. zone to the $\alpha$ state. As we have shown in section 5.2, further Bain strain relaxation proceeded with the development of either of two distinct precipitate morphologies depending on the thermal history of an alloy. For the continuously cooled alloys, as typically represented by air-cooled alloys, the relaxation process involved a direct formation of the saw-tooth type polytwin plates by nucleation and growth of twin domains inside the existing $\langle 100\rangle_{\gamma}$-oriented single domain plates and by concomitant segment-wise habit rotation. By contrast, for the quenched/aged alloys, the relaxation process involved habit plane rotation of individual precipitate plates in parallel with a reconfiguration of the plates into a macroscopic polytwin morphology. In this case, the twin domains introduced earlier in the aging process were found not stable with respect to global habit rotation of the precipitate plates.

Inasmuch as the difference in precipitate morphologies was caused by a change in the kinetic path of the decomposition. we considered it to be related directly to the difference in relative reaction time in the $(b c c+B 2)$ two phase field. As we indicated earlier, a prolonged 


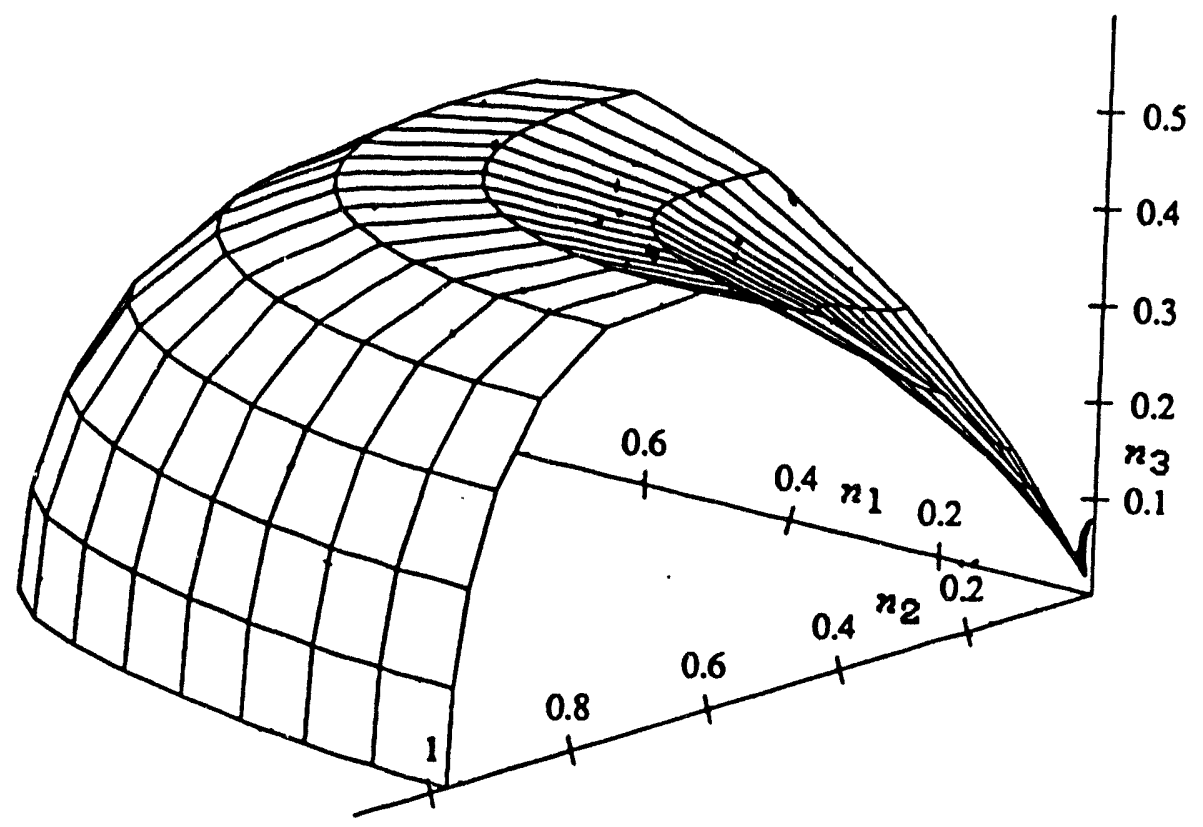

Figure 5.17: An octant of the elastic energy surface as a function of habit plane normals of a foc plate-like precipitate embedded in the $B 2$ matrix. Notice the local maximum along the [001] direction, indicating that the $\alpha^{\prime}$ state is elastically unstable. 
isostructural decomposition would lead to a significant coarsening of the $\mathrm{Cu}$-rich $\{001\}$ precipitate plates before their transformation into $\alpha^{*}$ precipitate plates. It is obvious that a global habit rotation of a large $\alpha^{\prime}$ precipitate plate is not kinetically favorable since it requires an extensive solute redistribution. In the presence of an effective twin formation mechanism, it would, instead, form a polytwin plate and rotate the habit planes segment-wise. The situation is different in the quenched/aged alloys. Since very fine precipitate plates, inherited from as-quenched states, are yet to grow and coarsen during the aging process, this will necessarily involve a reconstruction of the microstructures on a macroscopic scale. Evidently, one may not expect the shape-preserving local changes which occur in the air-cooled alloys.

We are now in a position to respond to a few important questions that our experimental observations bring forth: Firstly, as opposed to the individual precipitate plate with a globally rotated habit plane, what is the nature of the saw-tooth type polytwin plate in terms of the Bain strain relaxation ?. Closely related to this, what is the origin of the dissolution of twin domains in a globally rotated precipitate plate ?. In the following, we attempt to shed some light on these questions from the standpoint of energetics. Some detailed thoughts on the formation mechanism of polytwin plates will be given in section 5.3.2.3.

\subsubsection{Hablt Rotation of a Polytwin Plate and Comparison with a Single Domaln Plate Case.}

The observed morphology of the polytwin plates is unique which, in our survey of the literature, has never been discussed. The morphology is different from the theoretical one not only in terms of its appearance but also in its nature. To be more specific, the morphology does not gurantee the fulfillment of the invariant plane strain condition and accordingly, a polytwin plate with the observed morphology is always in an elastically constrained state unless its interface with matrix becomes incoherent. In order to understand this point more clearty, we start with a description of a theoretical morphology of a polytwin plate which is predicted from the elasticity theory of polytwin morphology [20-22] or equivalently 
from the theories of twinned martensite [23].

Theoretlcal Morphology of a Polytwin Plate

Invariant plane strain is known to be a concept of key importance in understanding the morphology of the two-phase mixture that arises from a phase transformation involving a crystal lattice reconstruction. The term refers to the distortion by which a crystal lattice plane (invariant plane) undergoes neither homogeneous distortion nor rigid body rotation during a phase transformation. When the stress free transformation strain is an invariant plane strain, the product phase lattice can maintain a complete coherency (or compatibility) with a parent phase lattice across an invariant plane without the expenditure of elastic strain energy. The Bain distortion accompanying a fcc to boc crystal lattice reconstruction is not an invariant plane strain, meaning that no lattice plane of the product phase crystal is kept coherent with the parent phase lattice unless homogeneous elastic distortion is involved. In this case, fulfillment of the invariant plane strain condition may be made possible by the formation of poly-twin complexes (or atternatively complexes of poly-translational domains) which consist of alfernating product phase domains of different variants.

In order to be consistent with the convention which we used to determine the habit planes in section 5.2, we consider a poly-twin plate made up of variant 3 and variant 2 which have the axes of Bain distortion parallel to $[001]_{y}$ and $[010]_{y}$ respectively. For such a polytwin plate. the total stress free transformation strain is given by

$$
\vec{\varepsilon}=2 \tilde{\varepsilon}^{0}(3)+(1-2) \hat{\varepsilon}^{0}(2)
$$

where $z$ is the volume traction of variant 3 and $\hat{\varepsilon}^{\circ}(3)$ and $\hat{\varepsilon}^{-0}(2)$ are the stress free transformation strain tensors of the variant 3 and 2, defined by eq. (5.7).

$$
\tilde{\varepsilon}^{0}(3)=\left(\begin{array}{ccc}
\varepsilon_{11}^{\circ} & 0 & 0 \\
0 & \varepsilon_{11}^{0} & 0 \\
0 & 0 & \varepsilon_{33}^{\circ}
\end{array}\right) \quad \hat{\varepsilon}_{(2)}^{0}=\left(\begin{array}{ccc}
\varepsilon_{11}^{0} & 0 & 0 \\
0 & \varepsilon_{33}^{\circ} & 0 \\
0 & 0 & \varepsilon_{11}^{0}
\end{array}\right)
$$


where $\varepsilon_{11}^{0}$ and $\varepsilon_{33}^{\circ}$ are given by eq. (5.4) for the Bain distortion we are considering. The volume dependent elastic strain energy of the polytwin plate which is characterized by eq. (5.6) and by an arbitrary habit normal $n$, is then given from eq. (2.27) to (2.30) with the strain tensor eq. (5.6) and the corresponding stress tensor substituted. With the resulting expression, the normalized effective strain energy $E_{\text {eff }}$ was defined: $E_{\text {eff }}=B\left(n, z_{e q}\right) / C_{11}$ (see eq.(2.27) for $B(n)$ ) where $z_{\text {eq }}$ is such that it extremizes $B(n, z)$ for a given habit normal $n$. $E_{\text {eff }}$

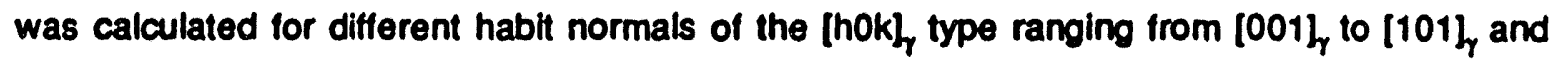
the results are shown in Fig. 5.18 along with the calculated results for a single domain $(z=1)$ plate. One can see that the strain energy of a polytwin plate is lower than that of a single domain plate for the entire range of habit normals and that it completely vanishes, therefore, the invariant plane strain condition is fulfilled at a particular habit normal $\left(n_{0}\right)$ and for a particular volume fraction $\left(z_{0}\right)$.

Elasticity theories of polytwin morphology and the crystallographic theory of twinned martensite systematically predict $\boldsymbol{n}_{0}$ and $z_{0}$ based on the concept of invariant plane strain. From the analytic expressions provided by elasticity theories [20-22], these quantities are found to be $n_{0}=(0.3998,0,0.9166)_{\gamma} /(1,0,2.29)_{\gamma}$ and $z_{0}=0.862^{\dagger}$, respectively ( $n_{0}$ for the single domain plate at the finite strain energy minimum is given by eq. (2.33) and (2.34); $n_{0}=$ $\left.(0.44241,0,0.89681)_{\gamma} / /(1,0,2.027)_{\gamma}\right)$. Remembering that the predicted invariant plane normal is calculated for a polytwin plate composed of variants 2 and 3 , one finds that it is quite different from the experimental result. The observed habit is of the (Ohk) $y$ type for the variant 3 domains and $\left(0 k^{\prime} h^{\prime}\right)_{y}$ type for the varaint 2 domains where $h<k$ and $h^{\prime}<k^{\circ}$. This clearly indicates that the observed morphology of the polytwin plate can not fulfill the invariant plane strain condition regardless of hk ratio. Within the context of the theories, the (Ohk) type invariant plane can only be attained for a polytwin plate consisting of variant 1 and 3. With variant 2 twin domains present, a saw-looth polytwin plate may achieve a complete relaxation of its strain energy either by the loss of coherency with the matrix or by

t The cystallographic theory of twinned martensite predicts $n_{0}=(0.38581,0.06385,0.82037)_{y}$ and $z_{0}=0.83284$. This discrepancy is due to the small strain approximation employed in the elasticity theory. 


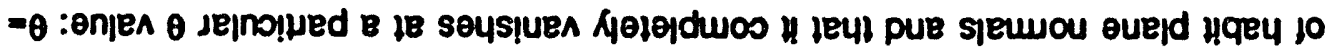

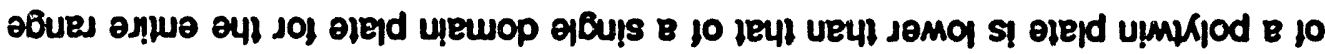

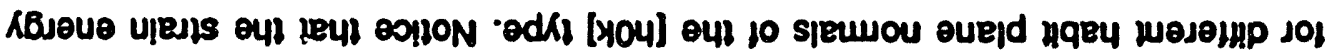

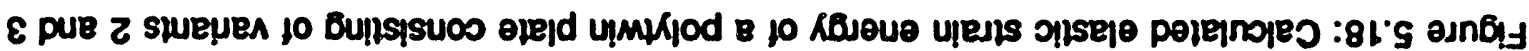
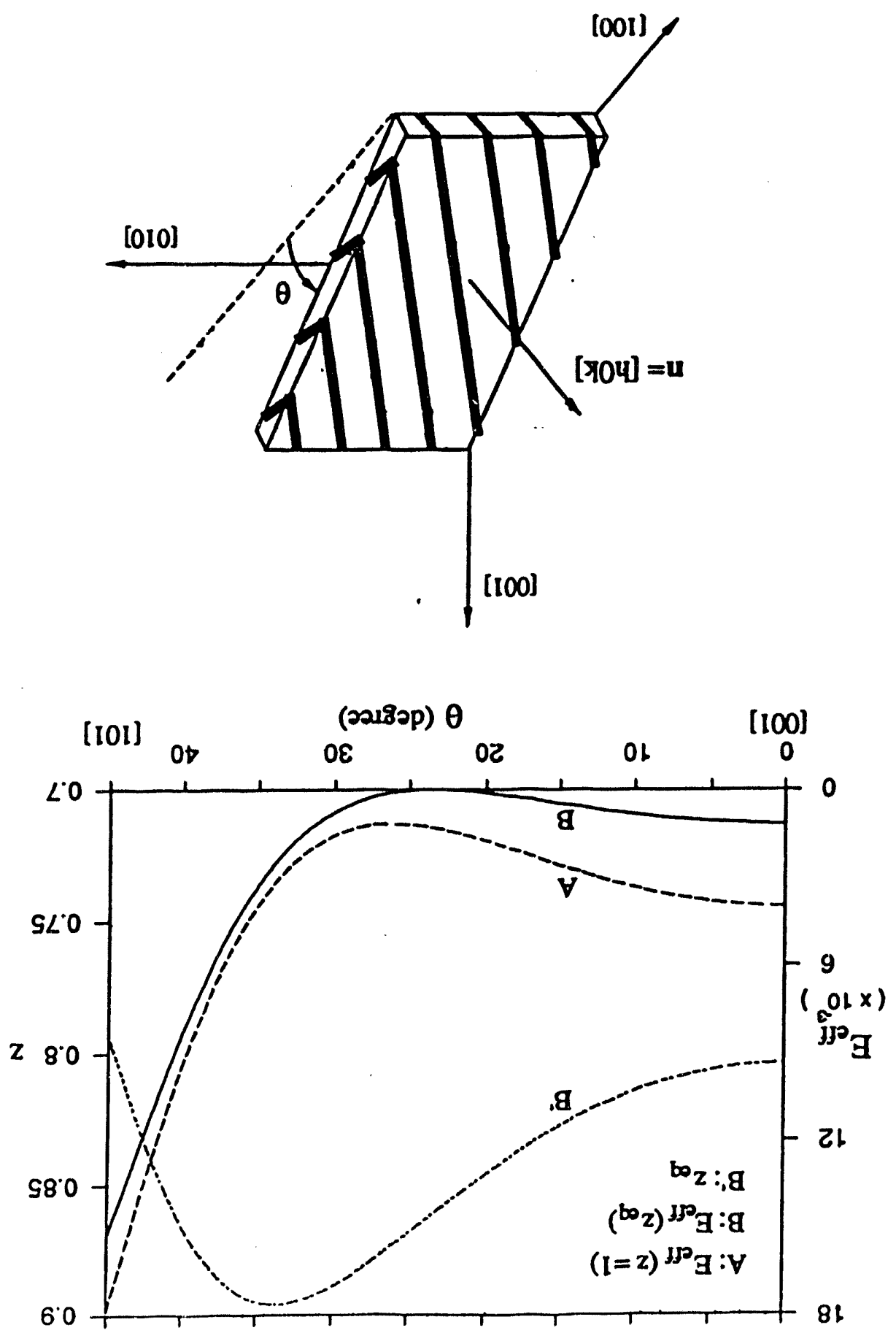
an additional introduction of secondary domains (either variant 1 twin domains or stacking faults). From the foregoing analysis, it is clear that great care should be taken when one compares an experimentally determined habit plane with the theoretically predicted one, since different permutations of similar numbers carry irreconcilable physical contents.

\section{Stability of a Varlant $(2+3)$ Composite Polytwin Plate with [Onk] Hablt.}

By use of the strain energy expression introduced above, a calculation was made for the

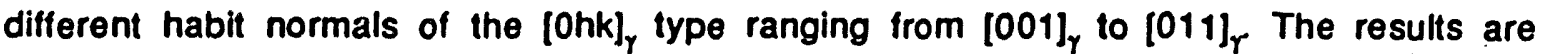
summarized in Fig. 5.19. One can notice a qualitatively different trend, as compared with the previous case, in the variation of the strain energy of a polytwin plate and of the volume fraction of the variant 3 with a change in habit normal. As the rotation angle $(\theta)$ increases, the strain energy of a polytwin plate remains constant while the energy difference $\left(E_{\text {eff }}(z=1)-E_{\text {eff }}\right.$ $\left.\left(z=z_{e q}\right)\right)$, the largest initially at the $[001\}_{\gamma}$ orientation, gradually decreases until it vanishes at the strain energy minimum of a single domain plate: $n_{0} / /(0,1,2.027)_{\gamma}$ In correspondence with this, the volume fraction of variant 3 monotonically increases (the volume fraction of variant 2 decreases) until it reaches $z=1$ where a polytwin plate switches to a single domain plate. This seems to explain qualitatively why variant 2 twin domains were rarely found in the globally rotated precipitate plates of the quenched/aged alloys.

To quantify the precise nature of the saw-tooth type polytwin plate beyond the qualitative discussions presented above, may require a refinement of the assumed physical model of a polytwin plate, given the discrepancy with the actual morphology. It is not attempted in the present work. Based on the foregoing analysis, it is concluded that the saw-tooth type polytwin plate is a kinetically conditioned metastable morphology. As we discussed earlier, the kinetic contributions were made by the intersection of the plates during incipient stages of the Bain transformation and the difficulty of mass transport for a global habit rotation.

\subsubsection{Formation Mechanism of the Saw-tooth Type Polytwin Plates.}



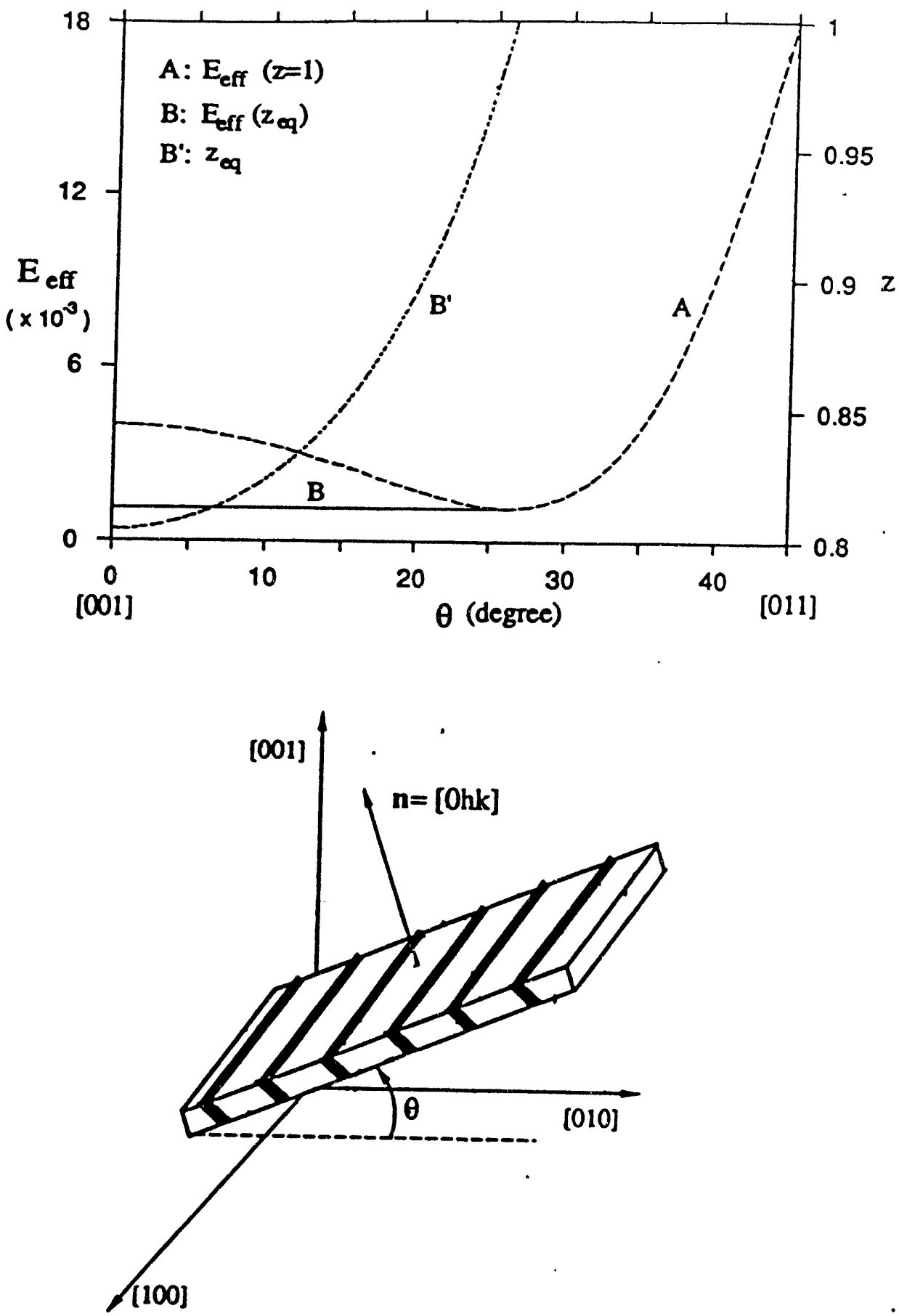

Figure 5.19: Calculated elastic strain energy of a polytwin plate consisting of variants 2 and 3 for different habit plane normals of the [Ohk] type. Notice that the strain energy of a polytwin plate gradually approaches that of a single domain plate until the energy difference completely vanishes at the strain energy minimum of a single domain plate. 
In understanding the formation mechanism of the saw-tooth type polytwin plates, one needs to address the following questions: 1. How do twin domains nucleate ?. 2. How do twins grow and how is their growth related to the segment-wise habit rotation ? 3. What are the atomic mechanisms responsible for habit rotation?. That is, what is the mechanism of interphase interface motion and how does diffusional atomic transport occur under the influence of stress field?, and so on. To answer these questions is a daunting task since it necessarily involves the scrutiny of related fundamental issues in the studies of diffusionless and of diffusional phase transformations. In the following, we limit our attention to and attempt to answer the first question on the basis of the most consistent observation we have made from the microstructures of the as-quenched and air-cooled alloys, that is, the intersection of the plates. We have suggested earlier that the intersection of plates plays an important role in nucleation of twin domains through the formation of stacking faults.

The $(100)_{\gamma}$ cross section of two infsersecting $<001>_{\gamma}$-oriented $\alpha^{*}(f c t)$ precipitate plates, is schematically depicted in Fig. 5.20. In the diagram, the tetragonality of the $\alpha^{\circ}$ precipitates is taken to be $0.8 \dot{3}$ and all the indices are given with respect to the variant 3 crystal lattice which is in Bain correspondence with the matrix lattice (upper and lower crystals are taken to be variant 2 and variant 3, respectively.). As one can nctice from the atomic configuration near the boundary between two variants, an immediate consequence of the impingement of a variant 2 plate with the broad face of a variant 3 plate is the genteration of a quasi misfit dislocation. The local Burgers vector (103) of the dislocation is defined to be $b=\frac{a^{\prime}}{2}[110]$ according to the SFIRI/ convention depicted in the diagram. As opposed to the conventional misfit dislocations which often reside at stable matrix/precipitate interfaces as an agent of misfit accommodation, the resulting dislocation has the following characteristics. Firstly, the dislocation is in a chemically homogeneous medium of a product phase. Secondly, it is subject to a highly strained environment in which the variant 3 crystal is elastically unstable and ready to relax the Bain strain. Under these circumstances, the dislocation may dissociate by the following reaction to produce a mobile Schockley partial which can then glide to create an intrinsic stacking fault on the (111) plane. 


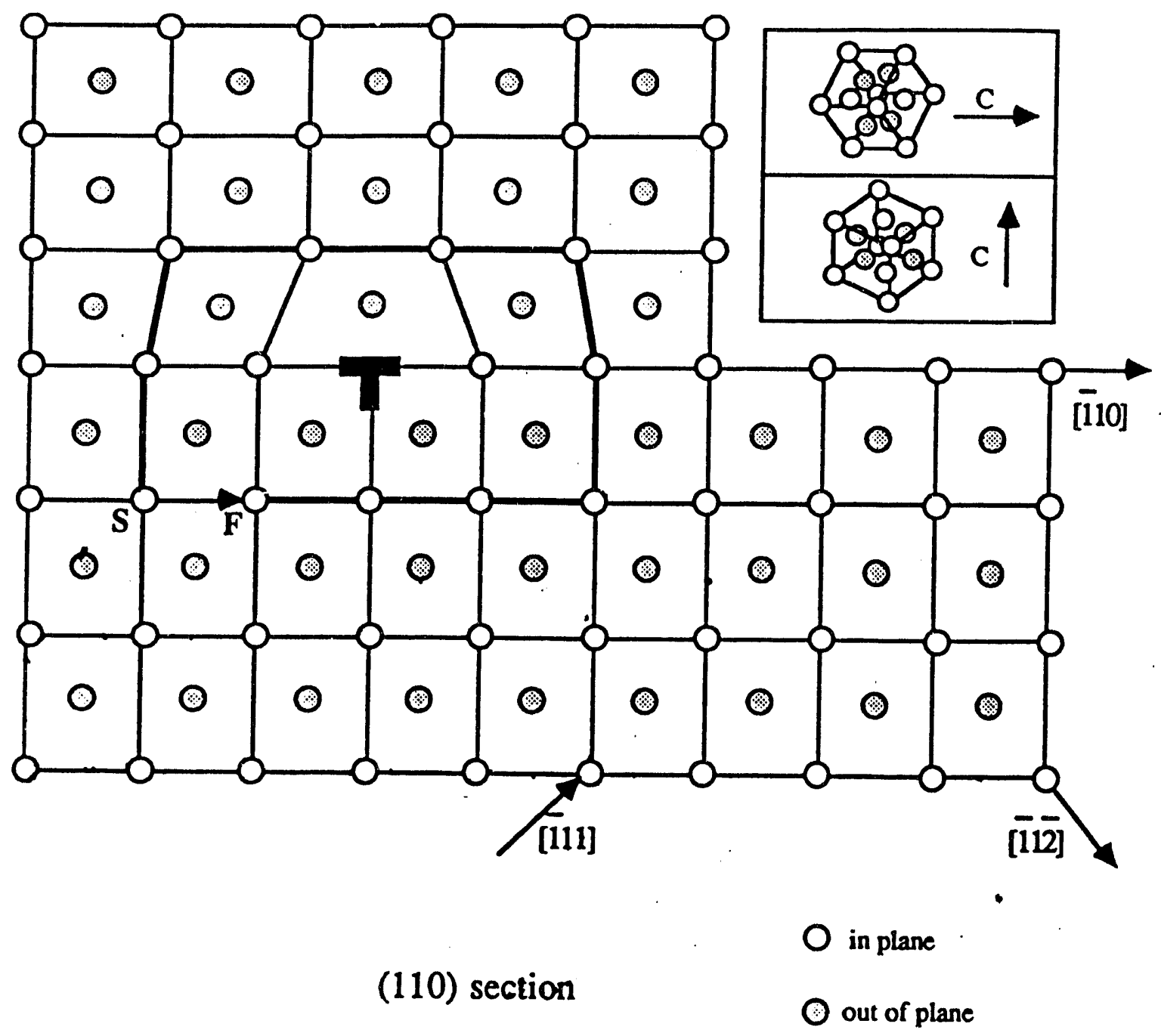

Figure 5.20: Schematic illustrating the generation of a quasi-misfit dislocation as a consequence of impingement of a variant 2 plate (upper crystal) with the broad face of a variant 3 plate (lower crystal). The dislocation dissociates to produce a mobile Shockley partial which then glides to create an intrinsic stacking fault on the (111) plane. 


$$
\frac{a^{\prime}}{2}[\overline{110}] \rightarrow \frac{a^{\prime}}{3}[\overline{111}]+\frac{a^{\prime}}{6}[\overline{112}]
$$

Inasmuch as the formation of a stacking fault has its origin in the generation of a quasi-misfit dislocation, the number of stacking faults due to a plate intersection depends on thickness of the impinging variant 2 plate. For example, when the variant 2 plate is as thick as of fifteen (001) varz planes (which may be the case in air-cooled alloys), three intrinsic stacking faults may be created with the average interval of six (111) vars planes. By a slight extension of the model, one can expect that an extrinsic fault may be produced when two thin plates of the variant 2 impinge on the variant 3 plate with one from either side.

Whether the described dislocation reaction can actually occur is thought to depend primarily on the elastic stress field inside the variant 3 precipitate plate. When perturbation due to impingment of the variant 2 plate is neglected, this means that the homogeneous stress field of the variant 3 plate should be able to provide the resolved shear stress which at least can overcome the resisting force associated with the creation of a stacking fault i.e. $\tau>$ $\gamma / b$ where $\tau, \gamma$ and $b$ are the resolved shear stress, the stacking fault energy and the magnitude of the Burgers vecior, respectively. By use of eq. (2.43), a general expression of the elastic stress tensor may be written as

$$
\sigma_{i j}=-C_{i j k l}\left(\varepsilon_{k l}^{\ell}-\varepsilon_{k l}^{\circ}\right)
$$

where $\varepsilon_{k l}^{l}$ is the tensor of the total homogeneous strain, given by eq. (2.44) and (2.45). For the variant 3 plate with the habit plane normal $n=[001]_{r}$ eq. (5.9) is reduced to

$$
\hat{\sigma}=\left(\begin{array}{ccc}
\sigma_{11} & 0 & 0 \\
0 & \sigma_{11} & 0 \\
0 & 0 & 0
\end{array}\right)
$$

where

$$
\sigma_{11}=-C_{11} \varepsilon_{11}^{0}\left(1+\frac{2 C_{12}}{C_{11}}\right)\left(1-\frac{C_{12}}{C_{11}}\right)
$$

Since $\varepsilon_{11}^{\circ}$ is negative in the case we are considering, the plate is in a planar tensile stress state with no stress component along the habit plane normal. The resolved shear stress on 
the $(\overline{1} 11)_{\alpha}$. plane along the $[\overline{1} 1 \overline{2}]_{\alpha}$. direction (nearly parallel to $(011)_{\gamma}$ and $[01 \overline{1}]_{\gamma}$ respectively) is then approximately $\tau \approx 0.5 \sigma_{11}$. Evaluation of $\tau$ leads to $\tau \approx 3 \times 10^{10} \mathrm{dyne} / \mathrm{cm}^{2}$. From the stacking fault energy of $\mathrm{Cu}\left(\gamma \sim 78 \mathrm{erg} / \mathrm{cm}^{2}\right)^{[104]}$ and $b=10^{-8} \mathrm{~cm}$, the minimally required resolved stress is estimated to be $\tau_{\min }-8 \times 10^{9}$ dyne/ $\mathrm{cm}^{2}$. From this, a conclusion is reached that intersection of plates and the ensuing dislocation reaction of eq. (5.8) are responsible for the nucleation of a twin domain (or formation of a stacking fault).

With regard to how stacking faults grow into twin domains, we presently have little understanding and it needs to be readdressed in carefully designed separate investigations, in view of importance of the problem. Within the limitation of our present work, it may sutfice to discuss briefly in what direction the answer is supposed to lie. We consider that it may be very difficult to account for growth of a twin domain exclusively in terms of diffusionless atomic motion i.e. conservative dislocation motion. According to Fig. 5.19, a polytwin plate with the (001) habit plane, is supposed to have twin domains of about $20 \%$ in volume fraction. In view of the extreme rapidity of dislocation motion, this means that, even in rapidlyquenched alloys, one would be able to observe the polytwin plates with nearly the predicted volume fraction of iwin domains, If diffusionless atomic motion were only responsible. Instead, we observed the predominant existence of stacking faults in Q1 alloys. ${ }^{\dagger}$ it is also very unlikely for a twin domain to grow via diffusional atomic motion alone, particularty inside an existing plate and in the presence of a mobile twinning dislocation. In light of this discussion, a possible mechanism might be one which involves non-conservative as well as conservative motion of the twinning Shockley partial dislocation which participated in the nucleation of a twin domain. To be more specific, the Schockley partial dislocation, unable to cross-slip, may advance to the next (111) plane by climb to add another layer of the stacking fault by glide motion. This alternating process of glide and climb motion does not appear to be unreasonable, given a relatively high reaction temperature and a finite clinb force due to

\footnotetext{
I In addition to a fow other aspects which we do not specify here, a pole mectranism [105,106), a widely accepted disfocation mechanism of doformation twinning in 100 crystals, seoms to be unsatistactory in this aspect. According to Cottrell and Biby's estimation [105], it takes only a fow nanoseconds for a single atomic twinned layer to grow by a pole mochanism.
} 
the stress field inside the precipitate plate (existence of the climb force can be clearly seen by use of the Peach-Koehler equation (103)). It may be interesting to note that, in the supposed mechanism, the plate intersection plays no role in twin growth except for the initial generation of a Schockley partial dislocation. This means that the growth of a twin domain may not be interrupted by the dissolution of the intersecting variant 2 plate.

\subsection{Conclusion}

Bain strain relaxation during precipitation of the Cu-rich $\alpha(f c c)$ phase in the hypereutectoid $\mathrm{Cu}-9.04 \mathrm{wt} \% \mathrm{Be}$ alloy was studied by transmission electron microscopy and the experimental findings were interpreted within the framework of the elasticity theory of a platelike precipitate. The major feature of the Bain strain relaxation was that it occurrs gradually along a decomposition path and through the interplay of two basic relaxation mechanisms, namely, habit plane rotation and the formation of polytwin domains. Depending on the kinetic path in the initial stages of the decomposition, these two mechanisms interplay in different manners to give rise to two different kinds of precipitate morphology. For quenched/aged alloys, the relaxation process involved global habit plane rotation of single domain plates in parallel with a reconfiguration into a macroscopic polytwin morphology. For continuously cooled alloys, as typically represented by air-cooled alloys, the relaxation process involved a direct formation of characteristic saw-tooth type polytwin plates by nucleation and growth of twin domains inside the existing $\langle 100\rangle_{\gamma}$-oriented single domain plates and by concomitant segment-wise habit rotation. From habit plane analysis, it was shown that, as opposed to the theoretical polytwin plate, a poly-twin plate with the saw-tooth morphology does not satisfy the invariant plane strain condition hence is not stress-free. The lack of twin domains in a globally rotated precipitate plate was explained along the same line of reasoning. From analysis of conventional TEM and HRTEM micrographs of the asquenched states, it was concluded that the intersection of plates is directly responsible for the nucleation of a twin domain in the saw-tooth plate and it was explained in terms of a dislocation model. It was also proposed that growth of a twin domain might involve 
$b$

solejd

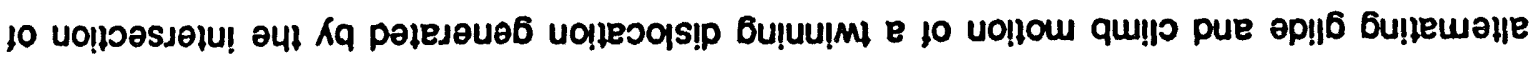




\section{Summary}

!

Unlike in displacive phase transformations, crystal lattice reconstruction during diffusional transformations, often occurrs either as a consequence of or in conjunction with atomic redistribution processes such as atomic ordering and clustering. In the present thesis, we have studied the interplay of a crystal lattice reconstruction with diffusional atomic redistribution processes in terms of its consequences on the thermodynamic stability and the morphology of the transformed product. Two different kinds of diffusional transformations were considered which involve cubic to tetragonal crystal lattice reconstruction, namely. L1。 ordering ( $f c c$ to $f(t)$ and Bain transformation in off-stoichiometric B2 ordering alloys (bcc to fcc). We have examined three specific questions:

1) What are the effects of the tetragonal distortion accompanying the $L 1_{0}$ ordering on the thermodynamics of $L 1_{0}$ ordering and on the alloy phase equilibria involving $L 1_{0}$ phase ?.

2) What are the effects of the tetragonal distortion on the morphology of the L1, phase ?.

3) How the Bain strain relaxation during decomposition of off-stoichiometric B2 ordering alloys is achieved by concurrence of atomic ordering and composition separation?.

With regard to the first question, we have conducted a theoretical investigation by treating the problem a rigid lattice free energy model. The treatment concerns itself purely with removal of the elastic strain energy contributions from a rigid lattice free energy which prevent the relaxation of the strain free rigid lattice into the stress free one. A generalized formulation was proposed in a form which can be directly utilized to study incoherent two phase equilibria. By employing the SCW mean field model as a rigid lattice free energy 
model, we have applied the formulation to the case of our interest.

We have shown that the thermodynamic stability of the $L 1_{0}$ phase may be significantly influenced by the relaxation of the tetragonal lattice distortion, depending on the magnitude of the associated negative strain energy relative to the competing positive entropic contribution in the free energy of the stress free $\mathrm{LI}_{0}$ phase. In association with this, a criterion was established. It states that within the context of the mean field free energy model, the first order nature of the $L 1_{0}$ ordering is recovered when $\alpha\left(\frac{e}{\mid V\left(k^{0}\right)}\right)$ exceeds a threshold value (0.02083 ). A prediction was also advanced that the problem of a rigid lattice free energy model and thus of the neglect of the tetragonal distortion could be more serious for alloy systems with lower $\mathrm{LI}_{0}$ ordering transition temperatures (e.g. CuAu and InMg), given the same magnitude of the tetragonal distortion. By taking imto account the tetragonal distortion, we have calculated a prototype phase diagram' of the $<001\rangle^{*}$ special point ordered structures. The resulting phase diagram has displayed fundamentally different characteristics, as compared to the one due to a rigid lattice mean field model. It was found that the topological features of the phase diagram resemble those of the CuAu-CuAu and the InMg$\mathrm{In}_{3} \mathrm{Mg}$ side of the respective experimental phase diagrams. Such an agreement clearly suggests an important role of the tetragonal distortion, as opposed to that of many body interaction or equivalently of the composition-dependent interatomic potential which have been proposed to explain the same features in the existing theoretical work. The conclusions attained in this study are qualitative and a precise assessment of the role of tetragonal distortion should be made by use of a free energy model which takes into account correlation effects. It is easily expected that the effects of the tetragonal distortion would be more pronounced in such a free energy model since it will produce a reduced entropy term relative to the exaggerated mean field entropy.

As for the second question, we have conducted a TEM investigation of the single L1, phase microstructure by use of an Fe-45 at.\%Pd alloy. It was shown that essential morphological features such as the variant habit plane, the variant volume fraction and the 
shape of a variant plate are determined so as to best accommodate the tetragonal distortion accompanying the $\mathrm{L1}_{0}$ ordering. For the variant habit planes, we have shown unequivocally that $\{110\}$ planes are the unique optimum habit planes, in agreement with the theoretical prediction as well as with many of the existing experimental observations. As for the variant volume fraction, a morphological aspect of utmost importance in regard to the relaxation of the homogeneous transformation strain, a characteristic sequential change was observed with the progress of ordering. Initially from a three variant state, the variant volume fraction in a local region was found to vary gradually through a two variant state until a near monovariant state is reached. It was shown that theories of polytwin morphology shed little light on the nature of this sequential change. We have explained this in terms of a synergistic interplay between the variant volume fraction and the constraining ability of the ordering surroundings. This interplay was suggested to be particularly substantial when the geometry of the specimen does not provide a rigid constraining ability. Along the same line of reasoning. the buckling phenomenon was explained. In case of a two variant state, the shape of a variant plate embedded in the matrix of the other variant was found to be looplike with an aspect ratio of $0.38 \sim 0.5$. By use of the elasticity theory of the shape of a platelike precipitate, a calculation was made to result in a good agreement with the experimental result.

As to the last question, a TEM investigation was made to study the Bain strain relaxation during precipitation of the $\mathrm{Cu}$-rich $\alpha(f c c)$ phase in the hyper-eutectoid $\mathrm{Cu}-9.04 w 1 \% \mathrm{Be}$ alloy and the experimental findings were interpreted within the framework of the elasticity theory of a plate-like precipitate. The major feature of the Bain strain relaxation was that it occurrs gradually along a decomposition path and through the interplay of two basic relaxation mechanisms, namely, habit plane rotation and the formation of polytwin domains. Depending on the kinetic path in the initial stages of the decomposition, these two mechanisms interplay in different manners to give rise to two different kinds of precipitate morphology. For quenched/aged alloys, the relaxation process involved global habit plane rotation of single domain plates in parallel with a reconfiguration into a macroscopic polytwin 
morphology. For continuously cooled alloys, as typically represented by air-cooled alloys, the relaxation process involved a direct formation of characteristic saw-tooth type polytwin plates by nucleation and growth of twin domains inside the existing $\langle 100\rangle_{\gamma}$-oriented single domain plates and by concomitant segment-wise habit rotation. From habit plane analysis, it was shown that, as opposed to the theoretical polytwin plate, a poly-twin plate with the saw-tooth morphology does not satisfy the invariant plane strain condition hence is not stress-free. The lack of twin domains in a globally rotated precipitate plate was explained along the same line of reasoning. From analysis of conventional TEM and HRTEM micrographs of the asquenched states, a conclusion was reached that the intersection of plates is directly responsible for the nucleation of a twin domain in the saw-tooth plate. This was explained in terms of a dislocation model. According to the model, growth of a twin domain might involve alternating glide and climb motion of a twinning dislocation generated by the intersection of plates. 
1. B. W. Roberts, Acta Met. 2, 597 (1954).

2. W. Shockley, J. Chem. Phys., 6, 130 (1938).

3. Y. Y. LI, J. Chem. Phys., 17, 447 (1949).

4. C. M. van Baal, Physica, 64, 571 (1973)

5. D. de Fontaine and R. Kikuchi, NBS publication sp-496, 999 (1978).

6. J. M. Sanchez, D. deFontaine and W. Teitler, Phys. Rev. B, 26, 1465 (1982).

7. T. Mohri, J. M. Sanchez and D. de Fontaine, Acta Met., 33, 1171 (1985).

8. K. Binder, J. L. Lebowitz, M. K. Phani and M. H. Kalos, Acta Met., 29, 1655 (1981).

9. K. Binder, W. Kinzel and W. Selke, J. Magn. Magn. Mater., 31-34, 1445 (1983).

10. M. Tachiki and K. Teramoto, J. Phys. Chem. Solids, 27, 335 (1966).

11. T. Kajitani and H. E. Cook, Acta Met., 26, 1371 (1978).

12. C. Sigli and J. M. Sanchez, Calphad, 8, 221 (1984).

13. A. Zunger, S. H. Wei, A. A. MBaye and L. G. Ferreira, Acta Met., 36, 2239 (1988).

14. L. E. Tanner and H. J. Leamy. ' The Microstructure of Order-Disorder Transitions', in Proc. International Symposium of Order-Disorder Transition in Alloys, ed. by $\mathrm{H}$. Warlimont, Springer, Tubingen (1974).

15. M. Sugiyama, Ph. D. thesis, Dept. Material Physics, Osaka University (1985).

16. M. Sugiyama, R. Oshima and F. E. Fujita, Trans. JIM, 27, 719 (1986).

17. R. Oshima, M. Sugiyama and F. E. Fujita, Met. Trans., 19A, 803 (1988).

18. S. Muto, S. Takeda, R. Oshima and F. E. Fujita, Japan. J. Appl. Phys., 27, L1387 (1988).

19. S. Muto, R. Oshima and F. E. Fujita, Acta Met., 38, 685 (1990).

20. A. G. Khachaturyan and G. A. Shatalov, Sov. Phys. JETP, 29, 557 (1969).

21. A. L. Roitburd, Sov. Phys. Solid State, 10, 2870 (1969). 
22. A. L. Roitburd, Phys. Stat. Sol. , 16 (a), 329 (1973).

23. M. S. Wechsler, D. S. Lieberman, and T. A. Read, Trans. Met. Soc. AIME, 197, 1503 (1953).

24. A. G. Khachaturyan!, Theory of Structural Transformations in Solids, John Wiley \& Sons, New York (1983).

25. A. G. Khachaturyan, Sov. Phys. Solid State, 9, 2040 (1968).

26. A. G. Khachaturyan, Fiz. metal. metalloved., 13, 493 (1962).

27. A. G. Khachaturyan, Sov. Phys. Solid State, 5, 16 (1963).

28. A. G. Khachaturyan, Progress in Materials Science, ed. by B. Chalmers, J. W. Christian and T. B. Massalski, Pergamon Press, 22, 150 (1978).

29. Ortrud Kubaschewski, IRON- Binary Phase Diagram, Springer-Verlag, 88 (1982).

30. A. H. Geisler, J. H. Mallery and F. E. Steigert, Trans. Am. Inst. Min. Engrs, 194, 307 (1952).

31. K. Shimizu, Y. Minakami, H. Mitani and K. Otsuka, J. Japan Inst. Met., 34, 1108 (1970).

32. V. A. Phillips and L. E. Tanner, Acta Met., 21, 441 (1973).

33. R. J. Rioja and D. E. Laughlin, Acta Met., 28, 1301 (1980).

34. A. G. Khachaturyan and D. E. Laughlin, Acta Met., 38, 1823 (1990).

35. A. G. Khachaturyan, Sov. Phys. Solid State, 8, 2163 (1967).

36. D. J. Chakrabanti, D. E. Laughlin and L. E. Tanner, Bull. Alloy Phase Diagrams, 8, 269 (1987).

37. M. I. Zakharova and Ye. M. Amosov, Fiz. met. metalloved., 14, 559 (1962).

38. T. Tadaki, T. Sahara and K. Shimizu, Trans. Japan Inst. Met., 14, 401 (1973).

39. X. Auvray, Sc. D. Thesis, Univ. Rouen, France (1977).

40. L. D. Landau, Sov. Phys., 11, 26 and 545 (1937).

41. E. M. Lifshitz, J. Phys., 6, 61 and 251 (1942).

42. L. D. Landau and E. M. Lifshitz, Statistical Physics, vol.5, part 1, 3rd ed., revised and enlarged by E. M. Litshitz and L. P. Pitaevskil, Pergamon Press, Oxtord (1976).

43. S. M. Allen and J. W. Cahn, Bull. Alloy Phase Diagrams, 3, 287 (1982). 
44. K. Binder, Rep. Prog. Phys., 50, 783 (1987).

45. W. L. Bragg and E. J. Williams, Proc. Roy. Soc., A145, 699 (1934); A152, 231 (1935).

46. S. V. Semenovskaya, Phys. Stat. Sol., 64 (b), 291 and 627 (1974).

47. Y. Murakami, N. Nakanishi and S. Kachi, Acta Met., 19, 93 (1971).

48. A. G. Khachaturyan, T. F. Lindsey and J. W. Morris, Met. Trans., 19A, 249 (1988).

49. D. de Fontaine, Acta Met., 23, 553 (1975).

50. D. de Fontaine, Configurational Thermodynamics of Solid Solutions, Solid State Physics, 34, 73 (197.9).

51. C. P. Bean and D. S. Rodbell, Phys. Rev., 126, 104 (1962).

52. M. E. Lines and E. D. Jones, Phys. Rev., 139, A1313 (1965).

53. F. Reif, Fundamentals of Statistical and Thermal Physics, McGraw-Hill Intemational (1981).

54. E. A. Guggenheim, Proc. Roy. Soc., A148, 304 (1935).

55. H. A. Bethe, Proc. Roy. Soc., A150, 552 (1935).

56. R. Peierls, Proc. Roy. Soc., A154, 207 (1936).

57. J. G. Kirkwood, J. Chem. Phys., 6, 70 (1938).

58. R. Kikuchi, Phys. Rev., 81, 998 (1951).

59. M. A. Krivoglaz, The Theory of $X$-ray and Thermal Netron Scattering from Real Crystals, Plenum Press, New York (1969).

60. M. A. Krivoglaz and A. A. Smirnov, The Theory of Order-Disorder in Alloys., Amer. Elsevier Publ. Co., New York (1965).

61. R. A. Suris, Sov. Phys., Solid State, 4, 850 (1962).

62. A. G. Khachaturyan and V. N. Hairapetyan, Phys. Stat. Sol., 57 (b), 801 (1973).

63. S. H. Wen, E. Kostlan, M. Hong . A. G. Khachaturyan and J. W. Morris, Jr., Acta Met., 29. 1247 (1981).

64. J. A. Wert, Acta Met., 24, 65 (1976).

65. A. G. Khachaturyan and A. F. Rumynina, Phys. Stat. Sol. 45 (a), 393 (1978).

66. A. G. Khachaturyan, S. V. Semenovskaya and J.W. Morris, Jr., Acta Met., 36, 1563 (1988). 
67. A. L. Roitburd, Sov. Phys. Crystallography, 12, 499 (1968).

68. A. L. Roitburd and N. S. Kosenko, Phys. Stat. Sol., 35 (a), 735 (1976).

69. E. Kostlan and J. W. Morris, Jr., Acta Met., 35, 2167 (1987).

70. A. G. Khachaturyan, Sov. Phys. Solid State, 9, 2249 (1968).

71. A. G. Khachaturyan, private communication.

72. W. B. Pearson, A Handbook of Lattice Spacings and Structures of Metals and Alloys, Pergamon Press, Oxford (1967).

73. S. M. Allen and J. W. Cahn, Bull. Alloy Phase Diagrams, 3, 787 (1982).

74. P. Nash and M. F. Singleton, Bull. Alloy Phase Diagrams, 10, 258 (1989).

75. H. Okamoto, D. J. Chakrabarti, D. E. Laughlin and T. B. Massalski, Bull. Alloy Phase Diagrams, 8, 454 (1987).

76. A. A. Nayeb-Hashemi and J. B. Clark, Bull. Alloy Phase Diagrams, 6, 149 (1985).

77. E. Metcalfe and J. A. Leake, Acta Met., 23, 1935 (1975).

78. K. H. Hellewege, Landolt-Bömstein Numerical Data and Functional Relationships in Science and Technology, New Series III (2), Springer-Verlag, Berlin (1969).

79. M. Bessière, Y. Calvayrac, S. Lefebvre, D. Gratias and P. Centedese, J. de Phys., 47, 1961 (1986).

80. D. A. Badalyan and A. G. Khachaturyan, Sov. Phys. Solid State, 12, 346 (1970).

81. P. B. Hirsh, A. Howie, R. B. Nicholson, D. W. Pashley and M. J. Whelan, Electron Microscopy of Thin Crystals, Robert E Krieger Publ. Co., Huntington, New York (1977).

82. L. E. Tanner, Phil. Mag., 14, 111 (1966).

83. I. M. Robertson and C. M. Wayman, Phil. Mag., 48A, 629 (1983).

84. M. Sato, B. H. Grier, S. M. Shapiro and H. Miyajima, J. Phys. F: Met. Phys., 12, 2117 (1982).

85. J. M. Pennison, A. Bourret and Ph. Eurin, Acta Met., 19, 1195 (1971).

86. P. J. Fillingham, H. J. Leamy and L. E. Tanner Jectron Microscopy and the Structure of Materials, Univ. of Califomia Press (1972).

87. S. H. Wen, A. G. Khachaturyan, J. W. Morris, Jr., Met. Trans. 12A, 581 (1981).

88. Yu. D. Tyapkin and K. M. Yamaleev, Sov. Phys. Doklady, 9, 322 (1964). 
89. K. M. Yamaleev and Yu. D. Tyapkin, Fiz. met. metalloved., 19, 133 (1965).

90. P. Eurin, J. M. Penisson and A. Bourret, Acta Met., 21, 559 (1973).

91. L. M. Magat, A. S. Yermolenko, G. V. Ivanova, G. M. Makarova and Ya. S. Shur, Fiz. met. metalloved., 26, 511 (1968).

92. R. Smith . J. S. Bowless, Acta Met., 8, 405 (1960).

93. H. C. Tong and C. M. Wayman, Acta Met., 21, 138 (1973).

94. S. M. Allen and J. W. Cahn, Acta Met., 24, 425 (1976).

95. H. Ino, Acta Met., 26, 827 (1978).

96. H. Kubo and C. M. Wayman, Metal. Trans., 10A, 633 (1979).

97. H. Kubo and C. M. Wayman, Acta Met., 28, 395 (1980).

98. Y. D. Tyapkin and V. A. Golikov, Phys. Met. Metall., 35, 336(1973); 36, 1058 (1973).

99. V. A. Golikov and Y. D. Tyapkin, Phys. Met. Metall., 37, 322 (1974).

100. R. Z. Abdulov, V. I. Syutkina and O. D. Shashkov, Fiz metal. metalloved., 45, 118 (1978).

101. D. V. Sukhanov, T. S. Boyarshinova and O. D. Shashkov, Fiz. metal. metalloved., 68, 316 (1989):

102. Long-Oing Chen and A. G. Khachaturyan , Acta Met., 39, 2533 (1991).

103. J. P. Hirth, J. Lothe, Theory of Dislocations, McGraw-Hill (1968).

104. L. E. Murr, Interfacial Phenomena in Metals and Alloys, Addison-Wesley (1975).

105. A. H. Cottrell and B. A. Bilby, Phil. Mag., 42, 573 (1951).

106. J. A. Venables, Phil Mag., 6, 379 (1961). 


\section{Derivation of Equation (2.39) ${ }^{\dagger}$.}

By use of eq.'s (2.30) and (2.37), we first establish the following relation:

$$
\delta^{2} B(n)=\beta_{i j} \delta n_{i} \delta n_{j}
$$

where $n$ is defined by eq. (2.35). From the expression of $B(n)$ given by eq. $(2.27), \delta^{2} B(n)$ is found to be

$$
\begin{aligned}
& \delta^{2} B(n)=-\delta^{2}\left(n \widehat{\sigma}^{\circ} \hat{\Omega}(n) \widehat{\sigma}^{\circ} n\right) \\
& =-\left[\delta^{2} n \widetilde{\sigma} \hat{\Omega}\left(n_{0}\right) \widetilde{\sigma}^{0} n_{0}+n_{0} \widehat{\sigma}^{0} \hat{\Omega}\left(n_{0}\right) \sigma^{0} \delta^{2} n+\delta n \widetilde{\sigma}^{0} \hat{\Omega}\left(n_{0}\right) \widetilde{\sigma}^{0} \delta n\right. \\
& \left.+\delta n \widehat{\sigma}^{\circ} \hat{\delta}(n) \widehat{\sigma}^{0} n_{0}+n_{0} \widehat{\sigma}^{\circ} \delta \hat{\Omega}(n) \widehat{\sigma}^{\circ} \delta n+n_{0} \widehat{\sigma}^{0} \delta^{2} \hat{\Omega}(n) \widehat{\sigma}^{0} n_{0}\right]
\end{aligned}
$$

In order to determine the variations $\hat{\delta} \hat{\Omega}(n)$ and $\delta^{2} \hat{\Omega}(n)$, we may use the Taylor expansions (A.3) and (A.4) and the identity (A.S).

$$
\begin{aligned}
& \hat{\Omega}(n)=\hat{\Omega}\left(n_{0}\right)+\delta \hat{\Omega}(n)+\delta^{2} \hat{\Omega}(n)+\ldots \\
& \hat{\Omega}^{-1}(n)=\hat{\Omega}^{-1}\left(n_{0}\right)+\delta \hat{\Omega}^{-1}(n)+\delta^{2} \hat{\Omega}^{-1}(n)+\ldots \\
& \hat{\Omega}(n)=\left[\hat{\Omega}^{-1}(n)\right]^{-1}
\end{aligned}
$$

Substitution of eq. (A.4) into eq. (A.S) leads to

$$
\begin{aligned}
\hat{\Omega}(n) & =\left[\hat{\Omega}^{-1}(n)+\delta \hat{\Omega}^{-1}(n)+\delta^{2} \hat{\Omega}^{-1}(n)+\ldots\right]^{-1} \\
& =\left[\hat{\Omega}^{-1}\left(n_{0}\right)\left(I+\hat{\Omega}\left(n_{0}\right) \delta \hat{\Omega}^{-1}(n)+\hat{\Omega}\left(n_{0}\right) \delta^{2} \hat{\Omega}^{-1}(n)+\ldots\right)\right]^{-1} \\
& =\left[I+\hat{\Omega}\left(n_{0}\right) \delta \hat{\Omega}^{-1}(n)+\hat{\Omega}\left(n_{0}\right) \delta^{2} \hat{\Omega}^{-1}(n)+\ldots\right]^{-1} \hat{\Omega}\left(n_{0}\right)
\end{aligned}
$$

\footnotetext{
The derivation was made by extending the procodure originally doveloped by Khachaturyan and Rumynina during their thooretical anaysis of the shape of a martonsite lath in Fo-Ni alloys. [65]
} 
where the identity $(\widehat{X} \widehat{Y})^{-1}=\widehat{Y}^{-1} \widehat{X}^{-1}$ is used. Noting that $\widehat{\Omega}\left(n_{0}\right) \delta \widehat{\Omega}^{-1}(n), \widehat{\Omega}\left(n_{0}\right) \delta^{2} \widehat{\Omega}^{-1}(n) \ldots$, have the order of $\delta n, \delta_{n}^{2} \ldots$, one can rewrite eq. (A.6) into eq. (A.9) by applying the power series (eq. (A.7)) and the approximation (eq. (A.8)) to the first two terms in the square bracket of eq. (A.6).

$$
\begin{gathered}
1+r+r^{2}+\cdots=\frac{1}{1-r} \\
(1+r)^{x} \cong 1+r x \\
\hat{\Omega}(n)=\hat{\Omega}\left(n_{0}\right)-\hat{\Omega}\left(n_{0}\right) \delta \hat{\Omega}^{-1}(n) \hat{\Omega}\left(n_{0}\right)+\left(\hat{\Omega}\left(n_{0}\right) \delta \widehat{\Omega}^{-1}(n)\right)^{2} \hat{\Omega}\left(n_{0}\right) \\
-\hat{\Omega}\left(n_{0}\right) \delta^{2} \hat{\Omega}^{-1}(n) \hat{\Omega}\left(n_{0}\right)+\cdots
\end{gathered}
$$

By comparison of eq. (A.9) with eq. (A.3),

$$
\begin{aligned}
& \delta \hat{\Omega}(n)=-\hat{\Omega}\left(n_{0}\right) \delta \hat{\Omega}^{-1}(n) \hat{\Omega}\left(n_{0}\right) \\
& \delta^{2} \hat{\Omega}(n)=\left(\hat{\Omega}\left(n_{0}\right) \delta \hat{\Omega}^{-1}(n)\right)^{2} \hat{\Omega}\left(n_{0}\right)-\hat{\Omega}\left(n_{0}\right) \delta^{2} \hat{\Omega}^{-1}(n) \hat{\Omega}\left(n_{0}\right)
\end{aligned}
$$

Substitution of eq. (A.11) into (A.2) and use of the relation $\delta^{2} n=\frac{1}{2} \mid \delta n^{2} n_{0}$ (eq. (2.35)) lead to

$$
\begin{aligned}
& \delta^{2} B(n)=n_{0} \widehat{\sigma}^{0} \widehat{\Omega}\left(n_{0}\right) \sigma^{0} n_{0}|\delta n|^{2}-\delta n \widehat{\sigma}^{\circ} \widehat{\Omega}\left(n_{0}\right) \widehat{\sigma}^{0} \delta n \\
& +\delta n \widehat{\sigma}^{0} \hat{\Omega}\left(n_{0}\right) \delta \widehat{\Omega}^{-1}(n) \hat{\Omega}\left(n_{0}\right) \widehat{\sigma}^{0} n_{0}+n_{0} \widehat{\sigma}^{0} \widehat{\Omega}\left(n_{0}\right) \delta \hat{\Omega}^{-1}(n) \hat{\Omega}\left(n_{0}\right) \widetilde{\sigma}^{0} \delta n \\
& \left.-n_{0} \widehat{\sigma} \alpha \hat{\Omega}\left(n_{0}\right) \delta \hat{\Omega}^{-1}(n)\right)^{2} \hat{\Omega}\left(n_{0}\right) \widehat{\sigma}^{0} n_{0}+n_{0} \widehat{\sigma}^{0} \hat{\Omega}\left(n_{0}\right) \delta^{2} \widehat{\Omega}^{-1}(n) \hat{\Omega}\left(n_{0}\right) \tilde{\sigma}^{0} n_{0}
\end{aligned}
$$

From eq. (2.45),

$$
S=\hat{\Omega}\left(n_{0}\right) \widetilde{\sigma}^{0} n_{0} \text {, and } S^{+}=\left(\hat{\Omega}\left(n_{0}\right) \widetilde{\sigma}^{0} n_{0}\right)^{+}=\left(n_{0}\right)^{+}\left(\hat{\sigma}^{0}\right)^{+} \hat{\Omega}^{+}\left(n_{0}\right)
$$

where + denotes adjoint and the identity $(\widehat{\mathbf{X}} \hat{\mathbf{Y}})^{+}=\widehat{\mathbf{Y}}^{+} \widehat{\mathbf{X}}^{+}$is used. Since $\mathbf{S}$ and $\mathbf{S}^{+}$are vectors and $\widehat{\sigma}^{\circ}$ and $\hat{\Omega}\left(n_{0}\right)$ are symmetric, $S=S^{+}=n_{0} \widehat{\sigma}^{\circ} \widehat{\Omega}\left(n_{0}\right)$. Substituting this into eq. (A.12) gives

$$
\begin{aligned}
\delta^{2} B(n) & =S \widehat{\Omega}^{-1}\left(n_{0}\right) S|\delta n|^{2}-\delta n \widehat{\sigma}^{0} \hat{\Omega}\left(n_{0}\right) \hat{\sigma}^{0} \delta n+\delta n \widehat{\sigma}^{0} \hat{\Omega}\left(n_{0}\right) \delta \hat{\Omega}^{-1}(n) S \\
& +S \delta \hat{\Omega}^{-1}(n) \hat{\Omega}\left(n_{0}\right) \widehat{\sigma}^{0} \delta n-S \delta \hat{\Omega}^{-1}(n) \hat{\Omega}\left(n_{0}\right) \delta \hat{\Omega}^{-1}(n) S+S \delta^{2} \hat{\Omega}^{-1}(n) S
\end{aligned}
$$

In order to reduce eq. (A.14) further, we use the expression of $\hat{\Omega}^{-1}(n)$ given by eq. (2.29). It follows that: 


$$
\delta \Omega_{i j}^{-1} S_{j}=\lambda_{i k l j} S_{j} n_{l}^{0} \delta n_{k}+\lambda_{i k l j} S_{j} n_{k}^{0} \delta n_{l}
$$

This can be recast into eq. (A.15) by use of the identities $\lambda_{i j k l}=\lambda_{i j l k}=\lambda_{j i k l}=\lambda_{j i l k}=\lambda_{k l i j}$.

$$
\delta \Omega_{i j}^{-1} s_{j}=\left(\sigma_{i j}+\sigma_{i j}^{*+}\right) \delta n_{j}
$$

where $\sigma_{i j}=\lambda_{i j k} S_{k} n_{i}^{0}, \sigma_{i j}^{*}=\lambda_{i k l j} S_{k} n_{l}^{0}$. By taking the adjoint in either side of eq. (A.15), one can show that:

$$
S_{i} \delta \Omega_{i j}^{-1}=\delta n\left(\sigma_{i j}+\sigma_{i j}^{*}\right)
$$

The second variation $\delta^{2} \hat{\Omega}^{-1}(n)$ is similarly reduced to eq. (A.17) by use of the relation $\delta^{2} n=\frac{1}{2}|\delta n|^{2} n_{0}$.

$$
\delta^{2} \Omega_{i j}^{-1}=\lambda_{i k l j} \delta n_{k} \delta n_{l}-\Omega_{i j}^{-1}\left(n_{0}\right) \mid \delta n^{2}
$$

By substituting eq.'s (A.15), (A.16) and (A.17) into eq. (A.14) and rearranging the resulting expression into the form of eq. (A.1), one can attain $\beta_{i j}\left(n_{0}\right)$ given by eq. (2.39).

$$
\beta_{i j}\left(n_{0}\right)=\lambda_{i j k} S_{k} S_{l}-\left\{\sigma_{i k}^{*} \Omega_{k l} \sigma_{j l}^{*}+\sigma_{i k}^{*} \Omega_{k}\left(\hat{\sigma}-\hat{\sigma}^{0}\right)_{j}+\left(\hat{\sigma}-\hat{\sigma}^{0}\right)_{i k} \Omega_{k l} \sigma_{j l}^{*}+\left(\hat{\sigma}-\hat{\sigma}^{0}\right)_{i k} \Omega_{k}\left(\hat{\sigma}-\hat{\sigma}^{\circ}\right)_{k j}\right\}
$$

where $\Omega_{i j}^{-1}=\lambda_{i k j} n_{k}^{0} n_{i}^{0}, \sigma_{i j}^{0}=\lambda_{i j k} \varepsilon_{k_{1}}^{\circ}, S_{i}=\Omega_{i j} \sigma_{j k}^{0} n_{k}^{0}(\widehat{\sigma})_{i j}=\lambda_{i j k k} S_{k} n_{i}^{0}$ and $\left(\widehat{\sigma}^{*}\right)_{i j}=\lambda_{i k l j} S_{k} n_{i}^{0}$.

153 

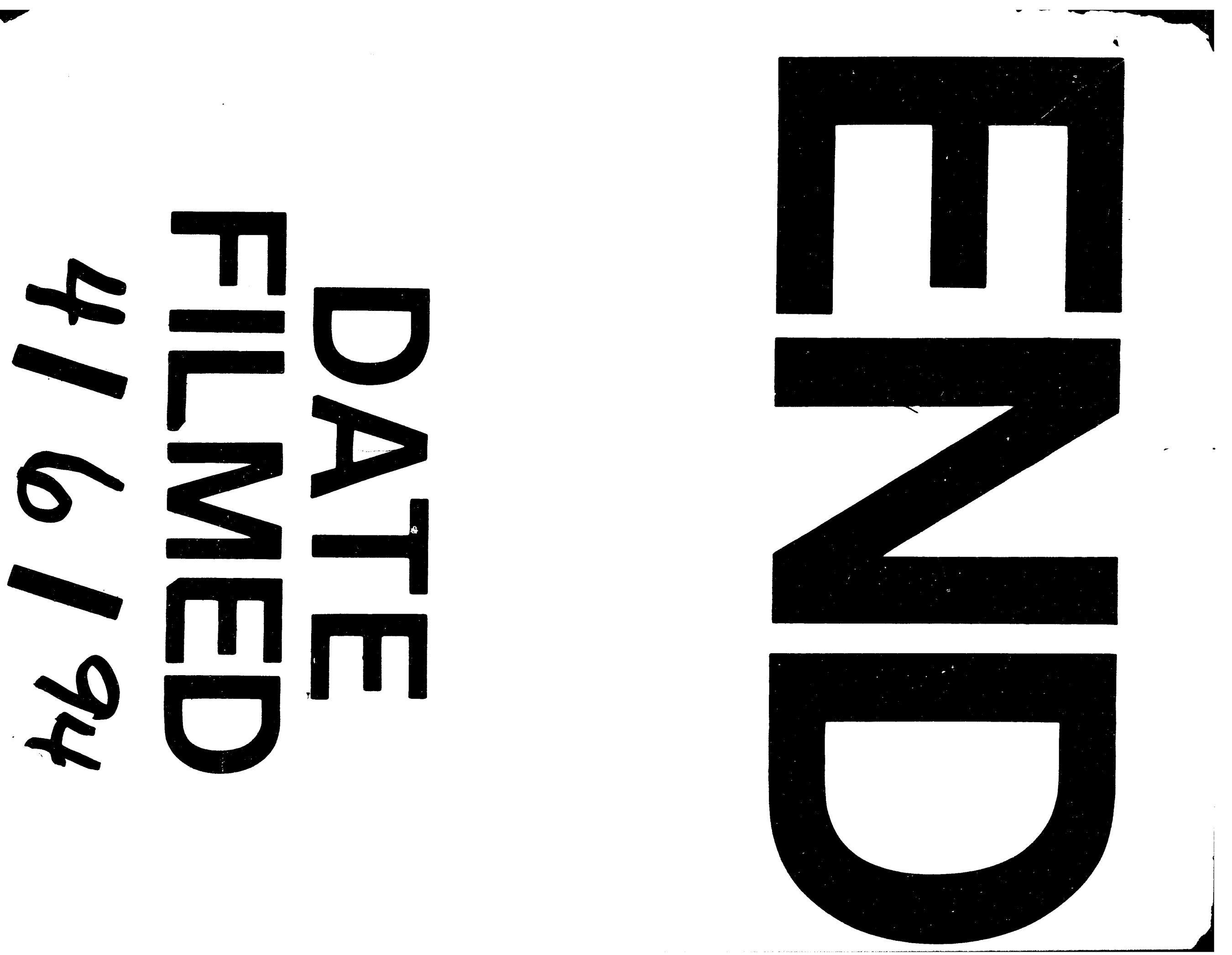
